\title{
Enantioselective Synthesis of Fused Isocoumarins via Palladium-Catalyzed Annulation of Alkyne-Tethered Malononitriles
}

\author{
Hui Zhang, ${ }^{\dagger}$ Weishuang Li, ${ }^{\dagger}$ Xu-Dong Hu, Wen-Bo Liu*
}

Sauvage Center for Molecular Sciences, Engineering Research Center of Organosilicon Compounds \& Materials (Ministry of Education), and College of Chemistry and Molecular Sciences, Wuhan University, Wuhan 430072, Hubei,

China.

E-mail: wenboliu@whu.edu.cn

\section{Table of Content}

Optimization of Reaction Conditions (Tables S1-S8) .2

Scheme S1. Formation of $\mathbf{2} \mathbf{a}^{\prime}$ and $2 \mathbf{a}^{\prime \prime}$ 7

Table S9. General Procedures for Preparation of Substrates 1 7

X-Ray Crystallographic Data of $\mathbf{2 a}$ 99

HPLC Spectra .17

NMR Spectra .36 


\section{Optimization of Reaction Conditions (Tables S1-S8)}

General procedure for condition optimizations: To a sealed tube equipped with a magnetic stirring bar were added [Pd] (0.005 mmol, $5 \mathrm{~mol} \%)$, ligand $(0.006 \mathrm{mmol}, 6 \mathrm{~mol} \%)$, and solvent in a glovebox. After the resultant mixture was stirred at room temperature for $0.5 \mathrm{~h}$, substrate $1 \mathrm{a}(34.2 \mathrm{mg}, 0.1 \mathrm{mmol}$, 1.0 equiv) was added. The tube was sealed and removed from the glovebox. Under an atmosphere of argon, $\mathrm{TsOH} \cdot \mathrm{H}_{2} \mathrm{O}$ (38.0 mg, $0.2 \mathrm{mmol}, 2.0$ equiv) was added and the mixture was stirred at the indicated temperature for $12 \mathrm{~h}$. The mixture was cooled to room temperature, filtered through a celite pad, and the celite pad was washed with DCM $(5 \mathrm{~mL} x$ 4). The collected filtrates were concentrated under reduced pressure to give a crude mixture. The yield was determined by ${ }^{1} \mathrm{H}$ NMR analysis of the crude mixture using dibromomethane as an internal standard. The er was determined by HPLC analysis (Chiralpak IA) after purification of the product using prep TLC.

\section{Table S1. Screening of Ligands.}

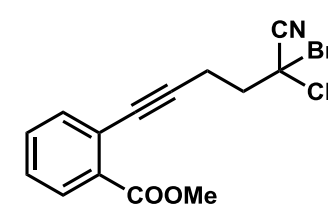

$1 a$

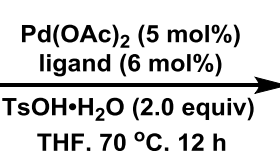
THF, $70^{\circ} \mathrm{C}, 12 \mathrm{~h}$

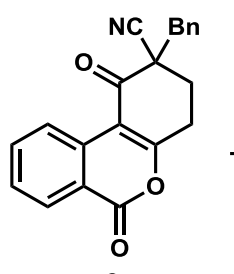

2a

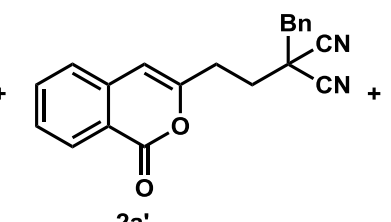

2a'

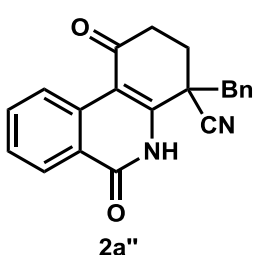

2a"<smiles>c1ccc(-c2ccccc2-c2c(-c3ccccc3)ccc3ccccc23)cc1</smiles>

L1<smiles>c1ccc(C[C@@H]2COC(c3ccccc3-c3ccccc3)=N2)cc1</smiles>

L5<smiles>CCC(C)C1COC(c2cc(C(F)(F)F)ccc2P(C)(=O)OC(C)(C)C)=N1</smiles>

$\mathrm{Ar}=3,5-\left(\mathrm{CF}_{3}\right)_{2} \mathrm{C}_{6} \mathrm{H}_{3}$

L9<smiles>CCC(C)[C@H]1COC(c2ccccn2)=N1</smiles>

L2<smiles>c1ccc(-c2ccccc2C2=N[C@H](c3ccccc3)CO2)cc1</smiles>

L6

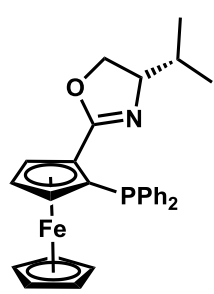

L10<smiles>CCC(C)[C@H]1COC(c2ccccc2-c2ccccc2)=N1</smiles>

L3<smiles>c1ccc(-c2ccccc2C2=N[C@H]3c4ccccc4C[C@H]3O2)cc1</smiles>

L7<smiles>CC(C)[C@H]1COC(c2ccccc2-c2ccccc2)=N1</smiles>

L4<smiles>COc1ccc(-c2ccccc2)c(C2=NC(C(C)(C)C)CO2)c1</smiles>

L8 


\begin{tabular}{cccccc}
\hline entry $^{a}$ & ligand & $\mathbf{2 a}(\%)^{b}$ & $\mathbf{2 a}^{\prime}(\%)^{b}$ & $\mathbf{2 a}^{\prime \prime}(\%)^{b}$ & er of $\mathbf{2} \mathbf{a}^{c}$ \\
\hline 1 & $\mathbf{L 1}$ & 57 & 19 & trace & $70: 30$ \\
2 & $\mathbf{L 2}$ & 40 & 24 & trace & $55: 45$ \\
3 & $\mathbf{L 3}$ & $64(60)$ & 6 & 9 & $93: 7$ \\
4 & $\mathbf{L 4}$ & 63 & trace & 8 & $78: 22$ \\
5 & $\mathbf{L 5}$ & 19 & 14 & 6 & $75: 25$ \\
6 & $\mathbf{L 6}$ & $76(65)$ & trace & 10 & $88: 12$ \\
7 & $\mathbf{L 7}$ & 35 & 9 & 8 & $91: 9$ \\
8 & $\mathbf{L 8}$ & $58(43)$ & trace & 9 & $94: 6$ \\
9 & $\mathbf{L 9}$ & trace & 13 & trace & $61: 39$ \\
10 & $\mathbf{L 1 0}$ & 41 & 13 & 6 & $70: 30$ \\
\hline
\end{tabular}

${ }^{a}$ Reactions conducted with $\mathrm{Pd}(\mathrm{OAc})_{2}(5 \mathrm{~mol} \%)$, ligand $(6 \mathrm{~mol} \%), \mathbf{1 a}(0.1 \mathrm{mmol}), \mathrm{TsOH} \cdot \mathrm{H}_{2} \mathrm{O}(2.0$ equiv) in THF (1.0 $\mathrm{mL}$ ) at $70{ }^{\circ} \mathrm{C}$ for $12 \mathrm{~h} .{ }^{b}$ Determined by ${ }^{1} \mathrm{H}$ NMR analysis of the crude mixture using dibromomethane as an internal standard with isolated yield in the parentheses. ${ }^{c}$ Determined by HPLC analysis (Chiralpak IA).

Table S2. Screening of Temperatures.

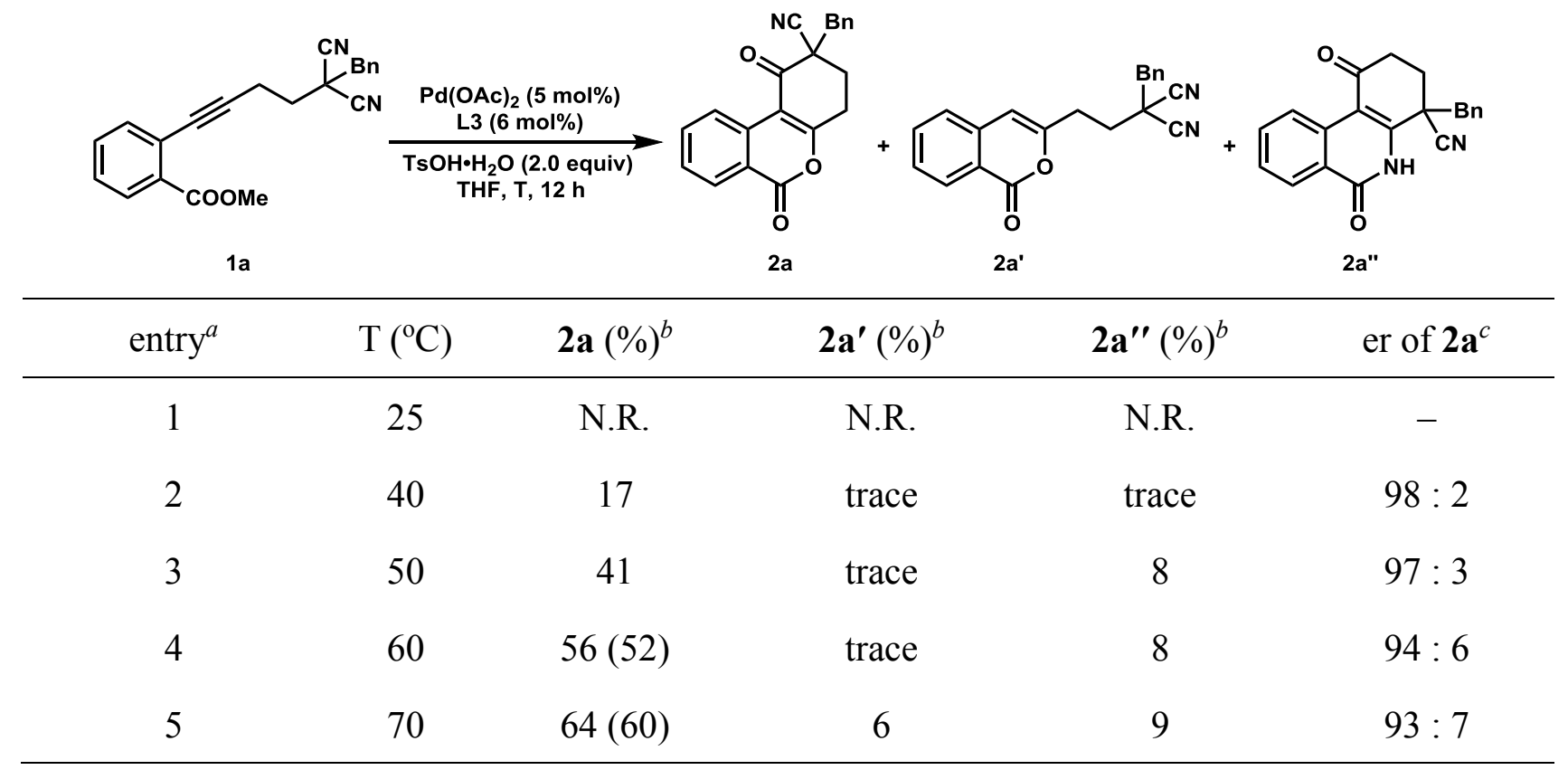

${ }^{a}$ Reactions conducted with $\mathrm{Pd}(\mathrm{OAc})_{2}(5 \mathrm{~mol} \%), \mathbf{L 3}(6 \mathrm{~mol} \%)$, $1 \mathrm{a}(0.1 \mathrm{mmol}), \mathrm{TsOH} \cdot \mathrm{H}_{2} \mathrm{O}$ (2.0 equiv) in THF (1.0 $\mathrm{mL}$ ) for $12 \mathrm{~h} .{ }^{b}$ Determined by ${ }^{1} \mathrm{H}$ NMR analysis of the crude mixture using dibromomethane as an internal standard with isolated yield in the parentheses. ${ }^{c}$ Determined by HPLC analysis (Chiralpak IA). 
Table S3. Screening of Palladium Sources and Catalyst Loadings.

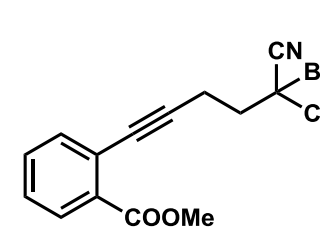

$1 \mathrm{a}$

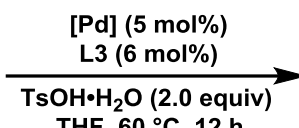
THF, $60^{\circ} \mathrm{C}, 12 \mathrm{~h}$<smiles>N#CC1(Br)CCc2oc(=O)c3ccccc3c2C1=O</smiles>

$2 a$<smiles>CC(C)(Br)CCc1cc2ccccc2c(=O)o1</smiles>

$2 a^{\prime}$

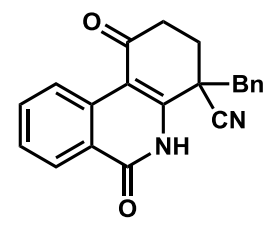

2a"

\begin{tabular}{cccccc}
\hline entry $^{a}$ & {$[\mathrm{Pd}]$} & $\mathbf{2 a}(\%)^{b}$ & $\mathbf{2 a}^{\prime}(\%)^{b}$ & $\mathbf{2 a}^{\prime \prime}(\%)^{b}$ & er of $\mathbf{2} \mathbf{a}^{c}$ \\
\hline 1 & $\mathrm{Pd}(\mathrm{OAc})_{2}$ & $56(52)$ & trace & 8 & $94: 6$ \\
2 & $\mathrm{Pd}(\mathrm{TFA})_{2}$ & $70(59)$ & 6 & 9 & $95: 5$ \\
$3^{d}$ & $\mathrm{Pd}(\mathrm{MeCN})_{2} \mathrm{Cl}_{2}$ & 52 & 7 & 11 & $93: 7$ \\
$4^{e}$ & $\mathrm{Pd}(\mathrm{TFA})_{2}$ & 70 & 6 & trace & $94: 6$ \\
\hline
\end{tabular}

${ }^{a}$ Reactions conducted with $[\mathrm{Pd}](5 \mathrm{~mol} \%), \mathbf{L 3}(6 \mathrm{~mol} \%), \mathbf{1 a}(0.1 \mathrm{mmol}), \mathrm{TsOH} \cdot \mathrm{H}_{2} \mathrm{O}(2.0$ equiv) in THF $(1.0 \mathrm{~mL})$ at $60{ }^{\circ} \mathrm{C}$ for $12 \mathrm{~h} .{ }^{b}$ Determined by ${ }^{1} \mathrm{H}$ NMR analysis of the crude mixture using dibromomethane as an internal standard with isolated yield in the parentheses. ${ }^{c}$ Determined by HPLC analysis (Chiralpak IA). ${ }^{d} 11$ mol\% AgOTf was added. ${ }^{e}$ With Pd(TFA) 2 (10 mol\%), and L3 (12 mol\%).

Table S4. Screening of Solvents.

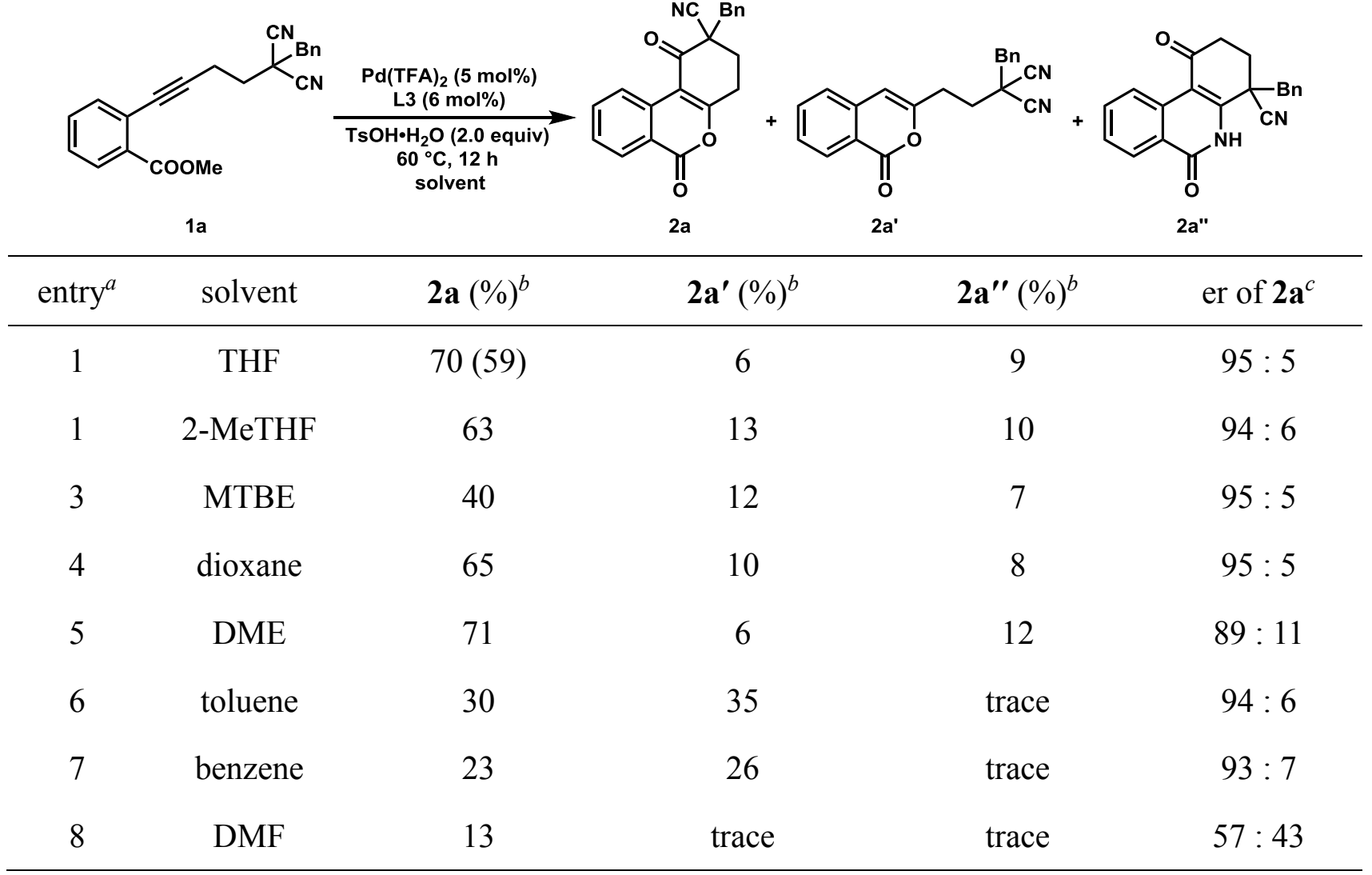


${ }^{a}$ Reactions conducted with $\mathrm{Pd}(\mathrm{TFA})_{2}(5 \mathrm{~mol} \%), \mathbf{L 3}(6 \mathrm{~mol} \%), \mathbf{1 a}(0.1 \mathrm{mmol}), \mathrm{TsOH} \cdot \mathrm{H}_{2} \mathrm{O}(2.0$ equiv) in solvent $(1.0$ $\mathrm{mL}$ ) at $60{ }^{\circ} \mathrm{C}$ for $12 \mathrm{~h} .{ }^{b}$ Determined by ${ }^{1} \mathrm{H}$ NMR analysis of the crude mixture using dibromomethane as an internal standard with isolated yield in the parentheses. ${ }^{c}$ Determined by HPLC analysis (Chiralpak IA).

Table S5. Screening of Acids.

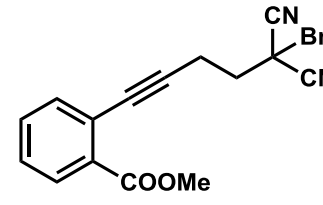

$1 \mathrm{a}$

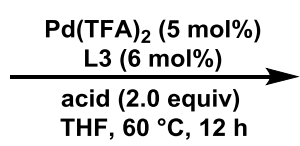
THF, $60^{\circ} \mathrm{C}, 12 \mathrm{~h}$

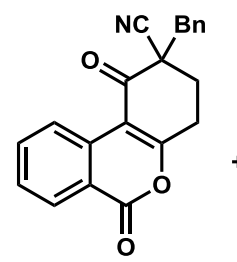

$2 a$<smiles>N#CC(Br)(Br)CCc1cc2ccccc2c(=O)o1</smiles>

2a'

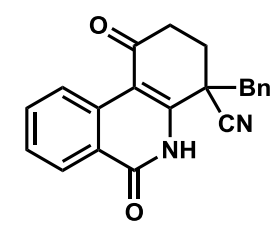

2a"

\begin{tabular}{|c|c|c|c|c|c|}
\hline entry $^{a}$ & acid & $\mathbf{2} \mathbf{a}(\%)^{b}$ & $\mathbf{2} \mathbf{a}^{\prime}(\%)^{b}$ & $2 \mathbf{a}^{\prime \prime}(\%)^{b}$ & er of $\mathbf{2} \mathbf{a}^{c}$ \\
\hline 1 & $\mathrm{TsOH} \cdot \mathrm{H}_{2} \mathrm{O}$ & $70(59)$ & 6 & 9 & $95: 5$ \\
\hline 2 & $\mathrm{MsOH}$ & 15 & 20 & N.D. & - \\
\hline 3 & $\mathrm{CF}_{3} \mathrm{SO}_{3} \mathrm{H}$ & 11 & N.D. & N.D. & - \\
\hline 4 & $\mathrm{NH}(\mathrm{Tf})_{2}$ & 25 & N.D. & 11 & $86: 14$ \\
\hline 5 & TFA & trace & trace & N.D. & - \\
\hline 6 & Amberlyst $^{\circledR} 15(50 \mathrm{mg})$ & N.R. & N.R. & N.R. & - \\
\hline $7^{d}$ & $\mathrm{TsOH} \cdot \mathrm{H}_{2} \mathrm{O}$ & 27 & trace & trace & $95: 5$ \\
\hline $8^{e}$ & $\mathrm{TsOH} \cdot \mathrm{H}_{2} \mathrm{O}$ & $72(68)$ & 6 & N.D. & $94: 6$ \\
\hline
\end{tabular}

${ }^{a}$ Reactions conducted with Pd(TFA) $2(5 \mathrm{~mol} \%), \mathbf{L 3}(6 \mathrm{~mol} \%), \mathbf{1 a}(0.1 \mathrm{mmol})$, acid $(2.0$ equiv) in THF $(1.0 \mathrm{~mL})$ at 60 ${ }^{\circ} \mathrm{C}$ for 12 h. ${ }^{b}$ Determined by ${ }^{1} \mathrm{H}$ NMR analysis of the crude mixture using dibromomethane as an internal standard with isolated yield in the parentheses. ${ }^{c}$ Determined by HPLC analysis (Chiralpak IA). ${ }^{d}$ With $\mathrm{TsOH} \cdot \mathrm{H}_{2} \mathrm{O}(1.0$ equiv). ${ }^{e}$ With $\mathrm{TsOH} \cdot \mathrm{H}_{2} \mathrm{O}$ (4.0 equiv).

Table S6. Screening of Additives.

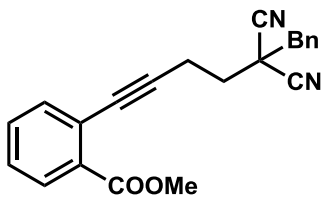

1a

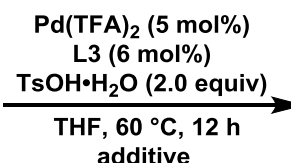

additive<smiles>N#CC1(Cc2ccccc2)CCc2oc(=O)c3ccccc3c2C1=O</smiles>

2a<smiles>CC(N)(Br)CCc1cc2ccccc2c(=O)o1</smiles>

2a'

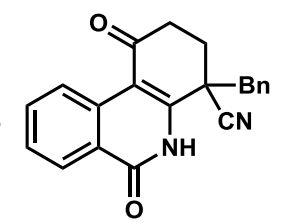

2a"

\begin{tabular}{cccccc}
\hline entry $^{a}$ & additive & $\mathbf{2 a}(\%)^{b}$ & $\mathbf{2 a}^{\prime}(\%)^{b}$ & $\mathbf{2 a}^{\prime \prime}(\%)^{b}$ & er of $\mathbf{2 a}^{c}$ \\
\hline 1 & - & $70(59)$ & 6 & 9 & $95: 5$
\end{tabular}




$\begin{array}{cccccc}2 & 4 \AA \mathrm{MS}(100 \mathrm{mg}) & \text { N.R. } & \text { N.R. } & \text { N.R. } & - \\ 3 & \mathrm{~B}(\mathrm{OH})_{3}(20 \mathrm{~mol} \%) & 64 & 9 & 10 & 95: 5 \\ 4 & \mathrm{ZnBr}_{2}(20 \mathrm{~mol} \%) & \text { N.R. } & \text { N.R. } & \text { N.R. } & - \\ 5 & \mathrm{Zn}(\mathrm{OTf})_{2}(20 \mathrm{~mol} \%) & 73 & 8 & 18 & 94: 6 \\ 6 & \mathrm{H}_{2} \mathrm{O}(5.0 \text { equiv }) & 28 & \text { trace } & \text { trace } & 91: 9\end{array}$

${ }^{a}$ Reactions conducted with $\mathrm{Pd}(\mathrm{TFA})_{2}(5 \mathrm{~mol} \%), \mathbf{L 3}(6 \mathrm{~mol} \%), \mathbf{1 a}(0.1 \mathrm{mmol}), \mathrm{TsOH} \cdot \mathrm{H}_{2} \mathrm{O}(2.0$ equiv) and additive in THF $(1.0 \mathrm{~mL})$ at $60{ }^{\circ} \mathrm{C}$ for $12 \mathrm{~h} .{ }^{b}$ Determined by ${ }^{1} \mathrm{H}$ NMR analysis of the crude mixture using dibromomethane as an internal standard with isolated yield in the parentheses. ${ }^{c}$ Determined by HPLC analysis (Chiralpak IA).

Table S7. Investigation of other protecting groups.

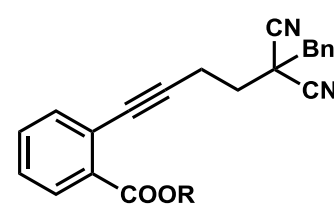

$1 \mathrm{a}$

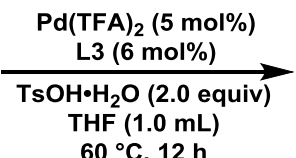

$60^{\circ} \mathrm{C}, 12 \mathrm{~h}$<smiles>N#CC1(Br)CCc2oc(=O)c3ccccc3c2C1=O</smiles>

$2 a$<smiles></smiles>

2a'

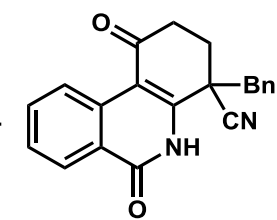

2a"

\begin{tabular}{cccccc}
\hline entry $^{a}$ & $\mathrm{R}$ & $\mathbf{2 a}$ yield $(\%)^{b}$ & $\mathbf{2 a}^{\prime}{\text { yield }(\%)^{b}}^{b}$ & $\mathbf{2 a}^{\prime \prime}$ yield $(\%)^{b}$ & er $^{c}$ \\
\hline 1 & $\mathrm{Me}$ & $70(59)$ & 6 & 9 & $95: 5$ \\
2 & $\mathrm{Et}$ & 57 & 7 & trace & $93: 7$ \\
3 & ${ }^{t} \mathrm{Bu}$ & 21 & 12 & trace & $87: 13$ \\
\hline
\end{tabular}

${ }^{a}$ Reactions conducted with $\mathrm{Pd}(\mathrm{TFA})_{2}(5 \mathrm{~mol} \%), \mathbf{L 3}(6 \mathrm{~mol} \%), \mathbf{1}(0.1 \mathrm{mmol}), \mathrm{TsOH} \cdot \mathrm{H}_{2} \mathrm{O}(2.0$ equiv) in THF $(1.0 \mathrm{~mL})$ at $60{ }^{\circ} \mathrm{C}$ for $12 \mathrm{~h} .{ }^{b}$ Determined by ${ }^{1} \mathrm{H}$ NMR analysis of the crude mixture using dibromomethane as an internal standard with isolated yield in the parentheses. ${ }^{c}$ Determined by HPLC analysis (Chiralpak IA).

Table S8. Screening of Concentrations.

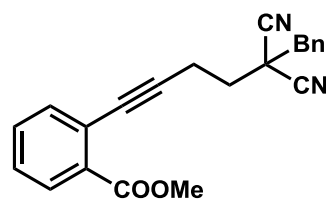

$1 a$

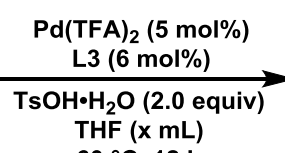
$60^{\circ} \mathrm{C}, 12 \mathrm{~h}$<smiles>C[CH2+]OC1=C(c2ccccc2C)C(=O)C(C)(Cc2ccccc2)CC1</smiles>

$2 \mathbf{a}$<smiles>CC(Br)(C#N)CCc1cc2ccccc2c(=O)o1</smiles>

2a'

\begin{tabular}{cccccc}
\hline entry $^{a}$ & $\mathbf{x}(\mathrm{mL})$ & $\mathbf{2 a}(\%)^{b}$ & $\mathbf{2 a}^{\prime}(\%)^{b}$ & $\mathbf{2 a}^{\prime \prime}(\%)^{b}$ & ${\text { er of } \mathbf{2} \mathbf{a}^{c}}^{{ }^{c}}$ \\
\hline 1 & 0.5 & $86(75)$ & 9 & 10 & $94: 6$ \\
2 & 1.0 & $70(59)$ & 6 & 9 & $95: 5$ \\
\hline
\end{tabular}




\begin{tabular}{cccccc}
\hline 3 & 2.0 & 17 & trace & N.D. & - \\
4 & 4.0 & 9 & 9 & N.D. & - \\
\hline
\end{tabular}

${ }^{a}$ Reactions conducted with $\mathrm{Pd}(\mathrm{TFA})_{2}(5 \mathrm{~mol} \%), \mathbf{L 3}(6 \mathrm{~mol} \%), \mathbf{1 a}(0.1 \mathrm{mmol}), \mathrm{TsOH} \cdot \mathrm{H}_{2} \mathrm{O}(2.0$ equiv) in THF (x mL) at $60{ }^{\circ} \mathrm{C}$ for $12 \mathrm{~h} .{ }^{b}$ Determined by ${ }^{1} \mathrm{H}$ NMR analysis of the crude mixture using dibromomethane as an internal standard with isolated yield in the parentheses. ${ }^{c}$ Determined by HPLC analysis (Chiralpak IA).

\section{Scheme S1. Formation of $2 a^{\prime}$ and $2 a^{\prime \prime}$}

During optimization of the conditions, by-product $\mathbf{2 a}^{\prime}$ formed from the protonation of alkenyl palladium intermediate and $\mathbf{2} \mathbf{a}^{\prime \prime}$ generated from translactamization process were also isolated.

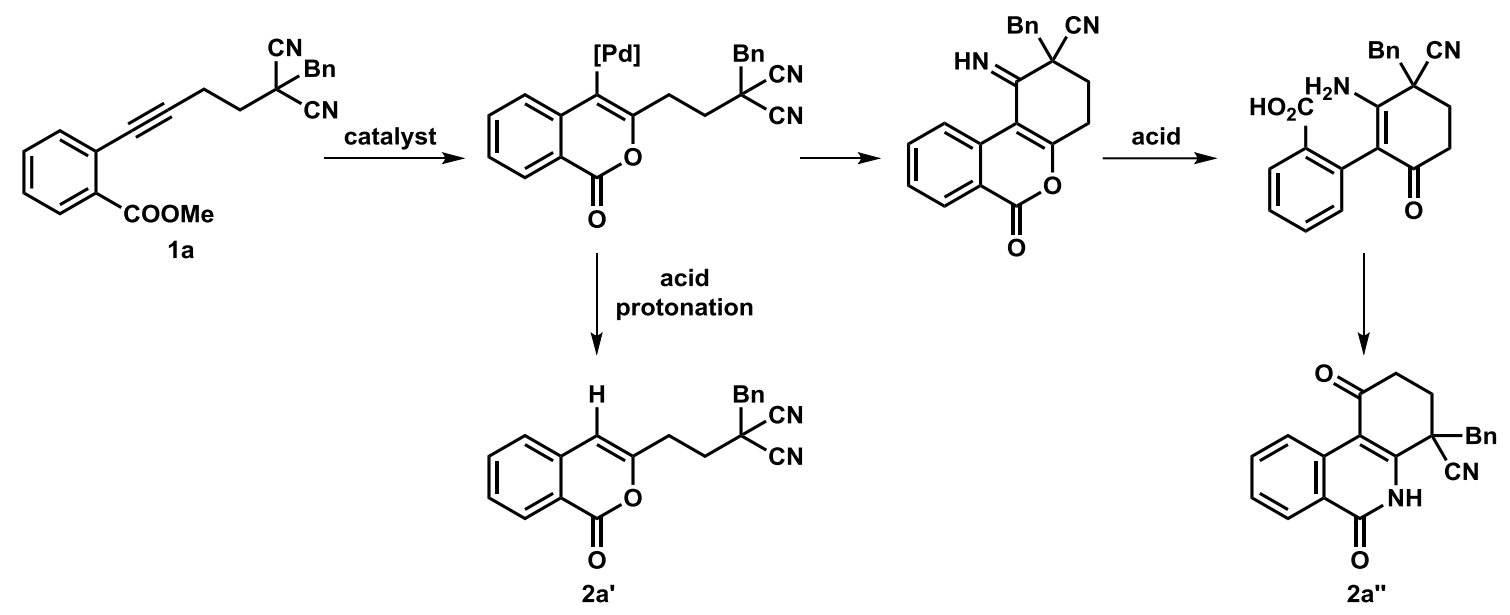

\section{Table S9. General Procedures for Preparation of Substrates 1}

General procedure A:

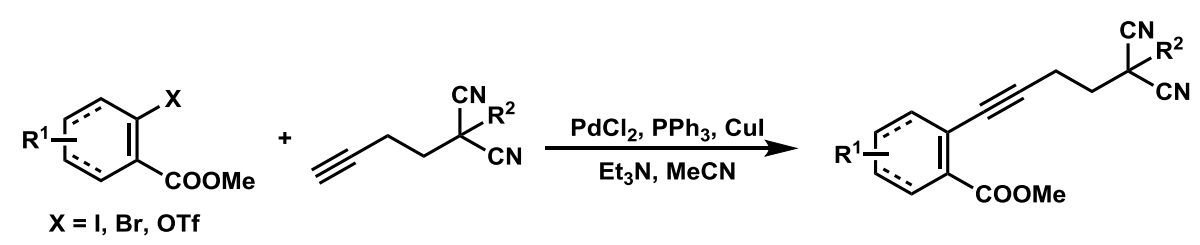

\begin{tabular}{|l|c|c|c|}
\hline Substrates & Structure & Substrates & Structure \\
\hline $\mathbf{1 a}$ & & $1 \mathbf{1 j}$
\end{tabular}




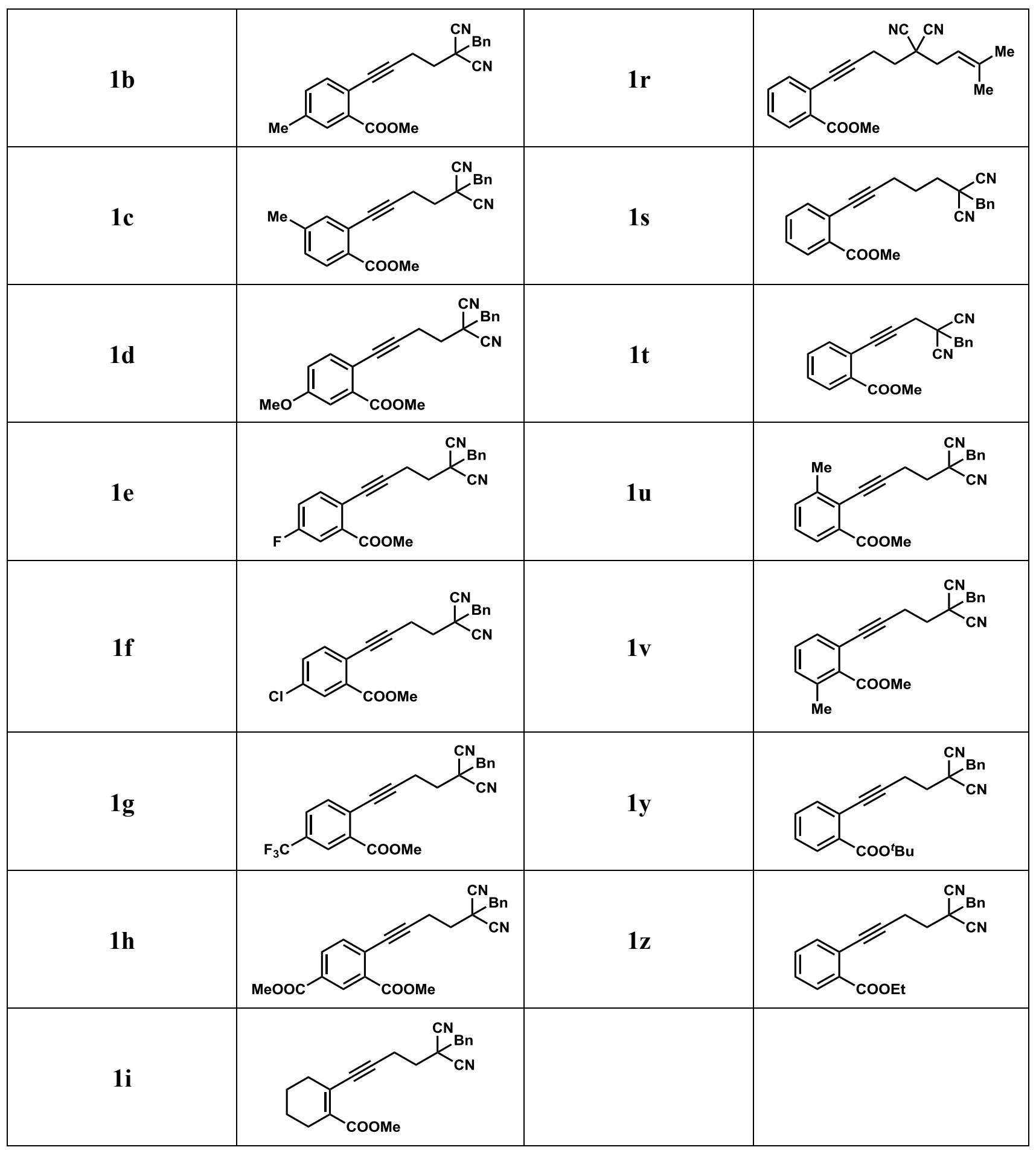

General procedure B:

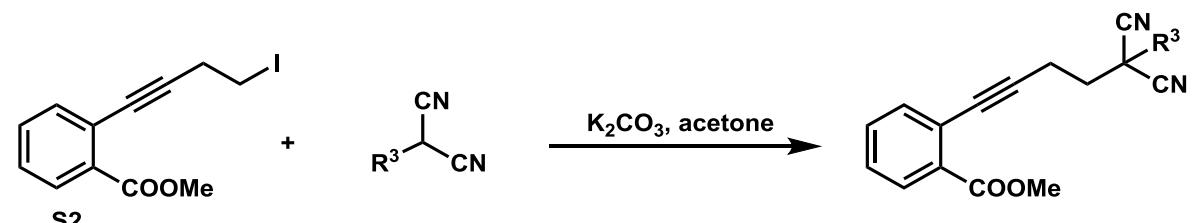

s2 


(n)

General procedure C:

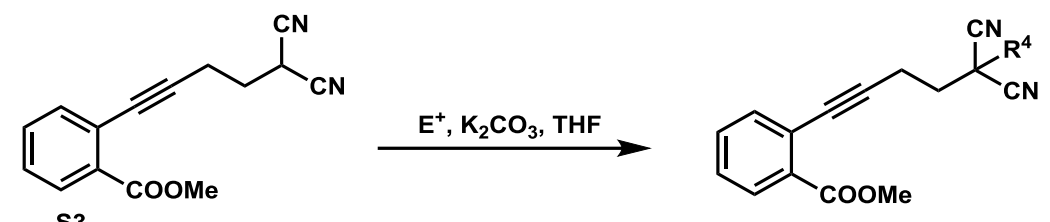

S3

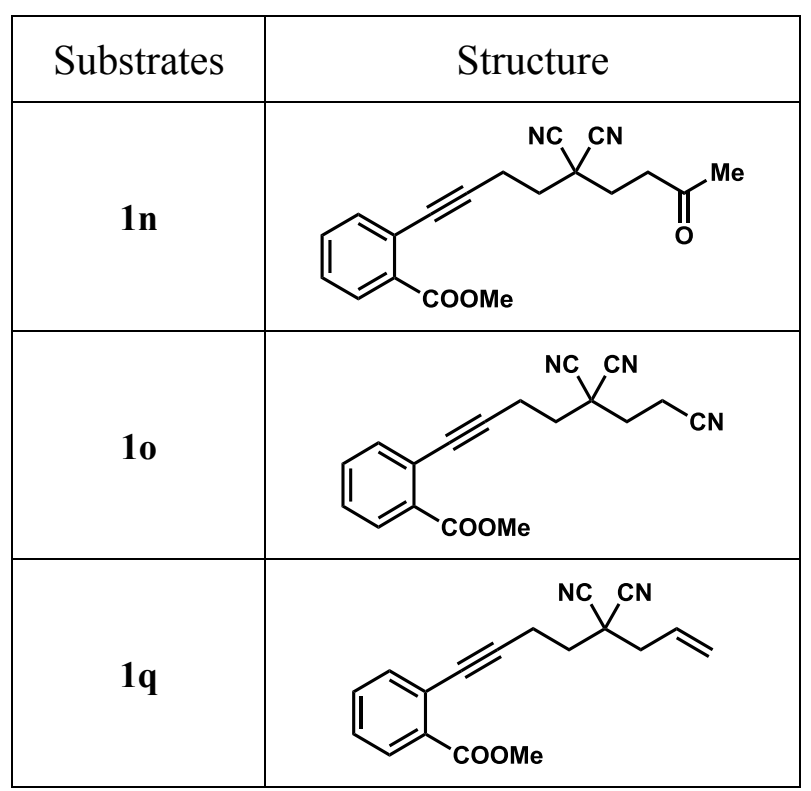

\section{X-Ray Crystallographic Data of 2a}

The crystals of 2a (>99.5:0.5 er) were grown by vapor diffusion method (EA and $n$-hexane) at $10{ }^{\circ} \mathrm{C}$. Data were collected on a Bruker SMART APEXII kappa diffractometer equipped with a CCD area detector and operated $(45 \mathrm{kV}, 0.65 \mathrm{~mA})$ to generate $\mathrm{Cu} \mathrm{K \alpha}$ radiation $(\lambda=1.5418 \AA)$. ORTEP picture of compound $\mathbf{2 a}$ was shown with the displacement ellipsoid drawn at $50 \%$ probability. 


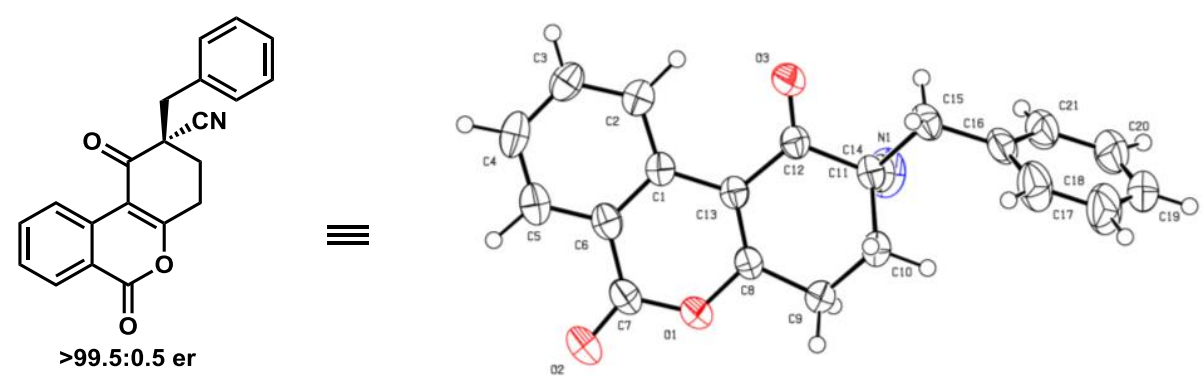

Table S10. Crystal data and structure refinement for $\mathbf{2 a}$.

Identification code

Empirical formula

Formula weight

Temperature

Wavelength

Crystal system

Space group

Unit cell dimensions

Volume

Z

Density (calculated)

Absorption coefficient

$\mathrm{F}(000)$

Crystal size

Theta range for data collection

Index ranges

Reflections collected

Independent reflections

Completeness to theta $=67.679^{\circ}$

Absorption correction

Max. and min. transmission

Refinement method

Data / restraints / parameters

Goodness-of-fit on $\mathrm{F}^{2}$
CCDC 2073023

$\mathrm{C}_{21} \mathrm{H}_{15} \mathrm{NO}_{3}$

329.34

$296.15 \mathrm{~K}$

$1.54178 \AA$

Monoclinic

P 1211

$a=8.1964(5) \AA$ $\alpha=90^{\circ}$.

$\mathrm{b}=6.6589(4) \AA$ $\beta=96.206(4)^{\circ}$.

$c=14.9770(8) \AA$ $\gamma=90^{\circ}$.

812.64(8) $\AA^{3}$

2

$1.346 \mathrm{Mg} / \mathrm{m}^{3}$

$0.734 \mathrm{~mm}^{-1}$

344

$0.08 \times 0.06 \times 0.04 \mathrm{~mm}^{3}$

5.429 to $67.842^{\circ}$.

$-9<=\mathrm{h}<=9,-8<=\mathrm{k}<=7,-16<=\mathrm{l}<=17$

5818

$2829[\mathrm{R}(\mathrm{int})=0.0254]$

$98.2 \%$

Semi-empirical from equivalents

0.7529 and 0.6246

Full-matrix least-squares on $\mathrm{F}^{2}$

2829 / 1 / 282

1.161 
Final $\mathrm{R}$ indices [I $>2 \operatorname{sigma}(\mathrm{I})]$

$\mathrm{R}$ indices (all data)

Absolute structure parameter

Extinction coefficient

Largest diff. peak and hole
$\mathrm{R} 1=0.0630, \mathrm{wR} 2=0.1740$

$\mathrm{R} 1=0.0651, \mathrm{wR} 2=0.1785$

$0.07(12)$

$\mathrm{n} / \mathrm{a}$

0.560 and -0.430 e. $\AA^{-3}$

Table S11. Atomic coordinates $\left(x \mathbf{1 0}^{4}\right)$ and equivalent isotropic displacement parameters $\left(\AA^{2} \mathbf{x}\right.$ $10^{3}$ ) for $2 a$. U(eq) is defined as one third of the trace of the orthogonalized $U^{i j}$ tensor.

\begin{tabular}{|c|c|c|c|c|}
\hline Atom & $\mathrm{x}$ & $\mathrm{y}$ & $\mathrm{z}$ & $\mathrm{U}(\mathrm{eq})$ \\
\hline $\mathrm{O}(1)$ & $301(3)$ & $5748(5)$ & $5329(2)$ & $46(1)$ \\
\hline $\mathrm{O}(3)$ & $5311(3)$ & $6147(6)$ & $7222(2)$ & $60(1)$ \\
\hline $\mathrm{O}(2)$ & $-41(4)$ & $5673(6)$ & $3851(2)$ & $65(1)$ \\
\hline $\mathrm{C}(1)$ & $3723(4)$ & $5878(4)$ & $5366(2)$ & $32(1)$ \\
\hline$C(13)$ & 2915(4) & $5892(5)$ & $6190(2)$ & $32(1)$ \\
\hline $\mathrm{C}(8)$ & $1255(4)$ & $5837(5)$ & $6133(2)$ & $36(1)$ \\
\hline$C(14)$ & $2657(4)$ & $3538(5)$ & $8047(2)$ & $40(1)$ \\
\hline $\mathrm{C}(7)$ & $931(4)$ & $5739(6)$ & $4520(2)$ & $44(1)$ \\
\hline $\mathrm{N}(1)$ & $2534(5)$ & $1842(6)$ & $8123(3)$ & $61(1)$ \\
\hline$C(12)$ & $3844(4)$ & $5945(5)$ & $7095(2)$ & $36(1)$ \\
\hline $\mathrm{C}(9)$ & $222(4)$ & $5875(6)$ & 6891(2) & $42(1)$ \\
\hline$C(6)$ & $2718(4)$ & $5827(5)$ & $4539(2)$ & $38(1)$ \\
\hline $\mathrm{C}(2)$ & $5428(4)$ & $5900(5)$ & $5332(2)$ & $39(1)$ \\
\hline $\mathrm{C}(15)$ & $3868(5)$ & $6622(6)$ & $8763(2)$ & $44(1)$ \\
\hline$C(16)$ & $3035(5)$ & $6524(6)$ & $9605(2)$ & $46(1)$ \\
\hline $\mathrm{C}(11)$ & $2857(4)$ & $5721(5)$ & $7922(2)$ & $35(1)$ \\
\hline $\mathrm{C}(3)$ & $6057(4)$ & $5886(6)$ & $4514(2)$ & $45(1)$ \\
\hline$C(10)$ & $1167(4)$ & $6728(5)$ & $7729(2)$ & $39(1)$ \\
\hline$C(4)$ & $5043(5)$ & $5852(6)$ & $3704(2)$ & $51(1)$ \\
\hline$C(5)$ & $3378(5)$ & $5816(6)$ & $3722(2)$ & $48(1)$ \\
\hline $\mathrm{C}(21)$ & $3150(6)$ & $4824(8)$ & $10154(3)$ & $58(1)$ \\
\hline $\mathrm{C}(17)$ & $2107(7)$ & $8132(8)$ & $9864(3)$ & $67(1)$ \\
\hline$C(20)$ & $2380(7)$ & $4725(10)$ & 10923(3) & $74(1)$ \\
\hline C(19) & $1471(6)$ & $6319(12)$ & $11172(3)$ & $80(2)$ \\
\hline $\mathrm{C}(18)$ & $1326(8)$ & $8010(10)$ & 10631(3) & $84(2)$ \\
\hline
\end{tabular}


Table S12. Bond lengths $[\AA ̊]$ and angles $\left[^{\circ}\right]$ for $2 a$.

\begin{tabular}{|c|c|}
\hline $\mathrm{O}(1)-\mathrm{C}(8)$ & $1.365(4)$ \\
\hline $\mathrm{O}(1)-\mathrm{C}(7)$ & $1.367(4)$ \\
\hline $\mathrm{O}(3)-\mathrm{C}(12)$ & $1.204(4)$ \\
\hline $\mathrm{O}(2)-\mathrm{C}(7)$ & $1.212(4)$ \\
\hline C(1)-C(13) & $1.462(4)$ \\
\hline$C(1)-C(6)$ & $1.411(4)$ \\
\hline $\mathrm{C}(1)-\mathrm{C}(2)$ & $1.403(4)$ \\
\hline C(13)-C(8) & $1.354(4)$ \\
\hline$C(13)-C(12)$ & $1.482(4)$ \\
\hline C(8)-C(9) & $1.488(4)$ \\
\hline $\mathrm{C}(14)-\mathrm{N}(1)$ & $1.141(6)$ \\
\hline $\mathrm{C}(14)-\mathrm{C}(11)$ & $1.477(5)$ \\
\hline$C(7)-C(6)$ & $1.463(5)$ \\
\hline $\mathrm{C}(12)-\mathrm{C}(11)$ & $1.558(4)$ \\
\hline C(9)-C(10) & $1.513(5)$ \\
\hline $\mathrm{C}(9)-\mathrm{H}(9 \mathrm{~A})$ & $1.03(6)$ \\
\hline C(9)-H(9B) & $1.03(6)$ \\
\hline$C(6)-C(5)$ & $1.391(5)$ \\
\hline$C(2)-C(3)$ & $1.380(4)$ \\
\hline $\mathrm{C}(2)-\mathrm{H}(2)$ & $1.03(6)$ \\
\hline$C(15)-C(16)$ & $1.499(5)$ \\
\hline$C(15)-C(11)$ & $1.552(4)$ \\
\hline $\mathrm{C}(15)-\mathrm{H}(15 \mathrm{~A})$ & $1.01(6)$ \\
\hline $\mathrm{C}(15)-\mathrm{H}(15 \mathrm{~B})$ & $0.95(6)$ \\
\hline$C(16)-C(21)$ & $1.396(6)$ \\
\hline$C(16)-C(17)$ & $1.392(6)$ \\
\hline $\mathrm{C}(11)-\mathrm{C}(10)$ & $1.538(5)$ \\
\hline $\mathrm{C}(3)-\mathrm{C}(4)$ & $1.394(6)$ \\
\hline $\mathrm{C}(3)-\mathrm{H}(3)$ & $0.96(5)$ \\
\hline $\mathrm{C}(10)-\mathrm{H}(10 \mathrm{~A})$ & $1.04(4)$ \\
\hline $\mathrm{C}(10)-\mathrm{H}(10 \mathrm{~B})$ & $0.98(5)$ \\
\hline$C(4)-C(5)$ & $1.368(6)$ \\
\hline $\mathrm{C}(4)-\mathrm{H}(4)$ & $0.97(5)$ \\
\hline $\mathrm{C}(5)-\mathrm{H}(5)$ & $1.05(5)$ \\
\hline $\mathrm{C}(21)-\mathrm{C}(20)$ & $1.373(6)$ \\
\hline $\mathrm{C}(21)-\mathrm{H}(21)$ & $0.91(6)$ \\
\hline
\end{tabular}




\begin{tabular}{|c|c|}
\hline$C(17)-C(18)$ & $1.376(8)$ \\
\hline $\mathrm{C}(17)-\mathrm{H}(17)$ & $0.91(7)$ \\
\hline $\mathrm{C}(20)-\mathrm{C}(19)$ & $1.372(9)$ \\
\hline $\mathrm{C}(20)-\mathrm{H}(20)$ & $0.92(8)$ \\
\hline $\mathrm{C}(19)-\mathrm{C}(18)$ & $1.385(10$ \\
\hline $\mathrm{C}(19)-\mathrm{H}(19)$ & $0.92(6)$ \\
\hline $\mathrm{C}(18)-\mathrm{H}(18)$ & 0.9300 \\
\hline $\mathrm{C}(8)-\mathrm{O}(1)-\mathrm{C}(7)$ & $123.1(3)$ \\
\hline $\mathrm{C}(6)-\mathrm{C}(1)-\mathrm{C}(13)$ & $117.7(3)$ \\
\hline $\mathrm{C}(2)-\mathrm{C}(1)-\mathrm{C}(13)$ & $125.0(3)$ \\
\hline $\mathrm{C}(2)-\mathrm{C}(1)-\mathrm{C}(6)$ & $117.2(3)$ \\
\hline $\mathrm{C}(1)-\mathrm{C}(13)-\mathrm{C}(12)$ & $122.5(3)$ \\
\hline $\mathrm{C}(8)-\mathrm{C}(13)-\mathrm{C}(1)$ & $119.4(3)$ \\
\hline $\mathrm{C}(8)-\mathrm{C}(13)-\mathrm{C}(12)$ & $118.2(3)$ \\
\hline $\mathrm{O}(1)-\mathrm{C}(8)-\mathrm{C}(9)$ & $110.8(3)$ \\
\hline $\mathrm{C}(13)-\mathrm{C}(8)-\mathrm{O}(1)$ & $122.2(3)$ \\
\hline$C(13)-C(8)-C(9)$ & $127.0(3)$ \\
\hline $\mathrm{N}(1)-\mathrm{C}(14)-\mathrm{C}(11)$ & $177.9(4)$ \\
\hline $\mathrm{O}(1)-\mathrm{C}(7)-\mathrm{C}(6)$ & 117.1(3) \\
\hline $\mathrm{O}(2)-\mathrm{C}(7)-\mathrm{O}(1)$ & $117.0(3)$ \\
\hline $\mathrm{O}(2)-\mathrm{C}(7)-\mathrm{C}(6)$ & $125.8(3)$ \\
\hline $\mathrm{O}(3)-\mathrm{C}(12)-\mathrm{C}(13)$ & $123.6(3)$ \\
\hline $\mathrm{O}(3)-\mathrm{C}(12)-\mathrm{C}(11)$ & $118.7(3)$ \\
\hline$C(13)-C(12)-C(11)$ & $117.7(2)$ \\
\hline $\mathrm{C}(8)-\mathrm{C}(9)-\mathrm{C}(10)$ & $110.9(3)$ \\
\hline $\mathrm{C}(8)-\mathrm{C}(9)-\mathrm{H}(9 \mathrm{~A})$ & $110(3)$ \\
\hline $\mathrm{C}(8)-\mathrm{C}(9)-\mathrm{H}(9 \mathrm{~B})$ & $107(3)$ \\
\hline $\mathrm{C}(10)-\mathrm{C}(9)-\mathrm{H}(9 \mathrm{~A})$ & $108(3)$ \\
\hline $\mathrm{C}(10)-\mathrm{C}(9)-\mathrm{H}(9 \mathrm{~B})$ & $112(3)$ \\
\hline $\mathrm{H}(9 \mathrm{~A})-\mathrm{C}(9)-\mathrm{H}(9 \mathrm{~B})$ & $109(4)$ \\
\hline $\mathrm{C}(1)-\mathrm{C}(6)-\mathrm{C}(7)$ & $120.4(3)$ \\
\hline$C(5)-C(6)-C(1)$ & $121.8(3)$ \\
\hline$C(5)-C(6)-C(7)$ & $117.8(3)$ \\
\hline $\mathrm{C}(1)-\mathrm{C}(2)-\mathrm{H}(2)$ & $118(3)$ \\
\hline$C(3)-C(2)-C(1)$ & $120.1(3)$ \\
\hline $\mathrm{C}(3)-\mathrm{C}(2)-\mathrm{H}(2)$ & $121(3)$ \\
\hline$C(16)-C(15)-C(11)$ & $114.5(3)$ \\
\hline
\end{tabular}




\begin{tabular}{|c|c|}
\hline$C(16)-C(15)-H(15 A)$ & $110(3)$ \\
\hline $\mathrm{C}(16)-\mathrm{C}(15)-\mathrm{H}(15 \mathrm{~B})$ & $108(3)$ \\
\hline $\mathrm{C}(11)-\mathrm{C}(15)-\mathrm{H}(15 \mathrm{~A})$ & $103(3)$ \\
\hline $\mathrm{C}(11)-\mathrm{C}(15)-\mathrm{H}(15 \mathrm{~B})$ & $106(3)$ \\
\hline $\mathrm{H}(15 \mathrm{~A})-\mathrm{C}(15)-\mathrm{H}(15 \mathrm{~B})$ & $116(5)$ \\
\hline$C(21)-C(16)-C(15)$ & $121.5(3)$ \\
\hline$C(17)-C(16)-C(15)$ & $120.8(4)$ \\
\hline$C(17)-C(16)-C(21)$ & $117.6(4)$ \\
\hline$C(14)-C(11)-C(12)$ & $105.6(3)$ \\
\hline$C(14)-C(11)-C(15)$ & $109.5(3)$ \\
\hline$C(14)-C(11)-C(10)$ & $110.1(3)$ \\
\hline$C(15)-C(11)-C(12)$ & $109.3(3)$ \\
\hline$C(10)-C(11)-C(12)$ & $110.1(2)$ \\
\hline$C(10)-C(11)-C(15)$ & $112.0(3)$ \\
\hline $\mathrm{C}(2)-\mathrm{C}(3)-\mathrm{C}(4)$ & $121.9(3)$ \\
\hline $\mathrm{C}(2)-\mathrm{C}(3)-\mathrm{H}(3)$ & $119(3)$ \\
\hline $\mathrm{C}(4)-\mathrm{C}(3)-\mathrm{H}(3)$ & $119(3)$ \\
\hline$C(9)-C(10)-C(11)$ & $111.2(3)$ \\
\hline $\mathrm{C}(9)-\mathrm{C}(10)-\mathrm{H}(10 \mathrm{~A})$ & $111(2)$ \\
\hline $\mathrm{C}(9)-\mathrm{C}(10)-\mathrm{H}(10 \mathrm{~B})$ & $110(3)$ \\
\hline $\mathrm{C}(11)-\mathrm{C}(10)-\mathrm{H}(10 \mathrm{~A})$ & $108(2)$ \\
\hline $\mathrm{C}(11)-\mathrm{C}(10)-\mathrm{H}(10 \mathrm{~B})$ & $108(3)$ \\
\hline $\mathrm{H}(10 \mathrm{~A})-\mathrm{C}(10)-\mathrm{H}(10 \mathrm{~B})$ & $107(4)$ \\
\hline $\mathrm{C}(3)-\mathrm{C}(4)-\mathrm{H}(4)$ & $123(3)$ \\
\hline$C(5)-C(4)-C(3)$ & $119.0(3)$ \\
\hline $\mathrm{C}(5)-\mathrm{C}(4)-\mathrm{H}(4)$ & $118(3)$ \\
\hline $\mathrm{C}(6)-\mathrm{C}(5)-\mathrm{H}(5)$ & $124(3)$ \\
\hline$C(4)-C(5)-C(6)$ & $120.0(3)$ \\
\hline $\mathrm{C}(4)-\mathrm{C}(5)-\mathrm{H}(5)$ & $115(3)$ \\
\hline $\mathrm{C}(16)-\mathrm{C}(21)-\mathrm{H}(21)$ & $121(4)$ \\
\hline$C(20)-C(21)-C(16)$ & $121.7(4)$ \\
\hline $\mathrm{C}(20)-\mathrm{C}(21)-\mathrm{H}(21)$ & $117(4)$ \\
\hline $\mathrm{C}(16)-\mathrm{C}(17)-\mathrm{H}(17)$ & $119(4)$ \\
\hline$C(18)-C(17)-C(16)$ & $120.3(5)$ \\
\hline $\mathrm{C}(18)-\mathrm{C}(17)-\mathrm{H}(17)$ & $121(4)$ \\
\hline $\mathrm{C}(21)-\mathrm{C}(20)-\mathrm{H}(20)$ & $129(5)$ \\
\hline$C(19)-C(20)-C(21)$ & $120.2(5)$ \\
\hline $\mathrm{C}(19)-\mathrm{C}(20)-\mathrm{H}(20)$ & $111(5)$ \\
\hline
\end{tabular}




$\begin{array}{ll}\mathrm{C}(20)-\mathrm{C}(19)-\mathrm{C}(18) & 119.0(4) \\ \mathrm{C}(20)-\mathrm{C}(19)-\mathrm{H}(19) & 121(4) \\ \mathrm{C}(18)-\mathrm{C}(19)-\mathrm{H}(19) & 120(4) \\ \mathrm{C}(17)-\mathrm{C}(18)-\mathrm{C}(19) & 121.2(5) \\ \mathrm{C}(17)-\mathrm{C}(18)-\mathrm{H}(18) & 119.4 \\ \mathrm{C}(19)-\mathrm{C}(18)-\mathrm{H}(18) & 119.4\end{array}$

Symmetry transformations used to generate equivalent atoms:

Table 13. Anisotropic displacement parameters $\left(\AA^{2} \times 10^{3}\right)$ for $2 a$. The anisotropic

displacement factor exponent takes the form: $-2 p^{2}\left[h^{2} a^{* 2} U^{11}+\ldots+2 h k a^{*} b^{*} U^{12}\right]$.

Atom $\quad \mathrm{U}^{11} \quad \mathrm{U}^{22} \quad \mathrm{U}^{33} \quad \mathrm{U}^{23} \quad \mathrm{U}^{13} \quad \mathrm{U}^{12}$

\begin{tabular}{|c|c|c|c|c|c|c|}
\hline $\mathrm{O}(1)$ & $39(1)$ & $64(2)$ & $34(1)$ & $-1(1)$ & $-2(1)$ & $-3(1)$ \\
\hline $\mathrm{O}(3)$ & $38(1)$ & $104(3)$ & $38(1)$ & $6(2)$ & $0(1)$ & $-11(1)$ \\
\hline $\mathrm{O}(2)$ & $58(2)$ & $97(2)$ & $36(1)$ & $-4(1)$ & $-10(1)$ & $0(2)$ \\
\hline $\mathrm{C}(1)$ & $41(2)$ & $24(1)$ & $32(1)$ & $2(1)$ & $5(1)$ & $0(1)$ \\
\hline C(13) & $38(1)$ & $29(1)$ & $30(1)$ & $1(1)$ & $4(1)$ & $-3(1)$ \\
\hline $\mathrm{C}(8)$ & $40(1)$ & $38(1)$ & $28(1)$ & $0(1)$ & $2(1)$ & $1(2)$ \\
\hline $\mathrm{C}(14)$ & $51(2)$ & $39(2)$ & $32(2)$ & $1(1)$ & $10(1)$ & $-2(1)$ \\
\hline$C(7)$ & $50(2)$ & $48(2)$ & $33(2)$ & $-2(2)$ & $-4(1)$ & $2(2)$ \\
\hline $\mathrm{N}(1)$ & $88(3)$ & $38(2)$ & $61(2)$ & $6(1)$ & $18(2)$ & $-2(2)$ \\
\hline$C(12)$ & $41(2)$ & $37(1)$ & $32(2)$ & $3(1)$ & $4(1)$ & $-1(1)$ \\
\hline$C(9)$ & $37(2)$ & $56(2)$ & $35(2)$ & $0(2)$ & $7(1)$ & $-2(2)$ \\
\hline$C(6)$ & $50(2)$ & $31(1)$ & $33(2)$ & $2(1)$ & $2(1)$ & $0(2)$ \\
\hline $\mathrm{C}(2)$ & $45(2)$ & $31(1)$ & $41(2)$ & $0(1)$ & $10(1)$ & $-2(1)$ \\
\hline$C(15)$ & $51(2)$ & $49(2)$ & $32(2)$ & $-3(1)$ & $-2(1)$ & $-7(2)$ \\
\hline$C(16)$ & $51(2)$ & $54(2)$ & $30(2)$ & $-6(1)$ & $-4(1)$ & $4(1)$ \\
\hline $\mathrm{C}(11)$ & $44(2)$ & $33(1)$ & $28(1)$ & $-1(1)$ & $3(1)$ & $-2(1)$ \\
\hline$C(3)$ & $50(2)$ & $36(1)$ & $51(2)$ & $3(2)$ & $19(1)$ & $-2(2)$ \\
\hline $\mathrm{C}(10)$ & $46(2)$ & $40(1)$ & $32(1)$ & $-1(1)$ & $8(1)$ & $1(1)$ \\
\hline$C(4)$ & $73(2)$ & $41(2)$ & $42(2)$ & $0(2)$ & $26(2)$ & $-4(2)$ \\
\hline$C(5)$ & $66(2)$ & $45(2)$ & $34(2)$ & $2(2)$ & $6(1)$ & $0(2)$ \\
\hline $\mathrm{C}(21)$ & $61(2)$ & $74(3)$ & $38(2)$ & $10(2)$ & $4(2)$ & $19(2)$ \\
\hline $\mathrm{C}(17)$ & $99(4)$ & $59(2)$ & $44(2)$ & $-10(2)$ & $6(2)$ & $21(2)$ \\
\hline $\mathrm{C}(20)$ & $75(3)$ & $105(4)$ & $42(2)$ & $21(2)$ & $7(2)$ & $18(3)$ \\
\hline $\mathrm{C}(19)$ & $64(3)$ & $142(6)$ & $37(2)$ & $0(2)$ & $11(2)$ & $21(3)$ \\
\hline
\end{tabular}


$\begin{array}{llllll}\mathrm{C}(18) & 103(4) & 103(4) & 48(2) & -18(2) & 14(3)\end{array}$

Table 14. Hydrogen coordinates $\left(x 1^{4}\right)$ and isotropic displacement parameters $\left(\AA^{2} \times 1^{3}\right)$ for 2 a.

\begin{tabular}{lcccc}
\hline Atom & $\mathrm{x}$ & $\mathrm{y}$ & $\mathrm{z}$ & $\mathrm{U}(\mathrm{eq})$ \\
& & & & \\
& & & & \\
$\mathrm{H}(18)$ & 688 & 9082 & 10788 & 101 \\
$\mathrm{H}(4)$ & $5460(60)$ & $5950(90)$ & $3130(30)$ & $55(11)$ \\
$\mathrm{H}(10 \mathrm{~A})$ & $530(50)$ & $6540(70)$ & $8290(30)$ & $42(10)$ \\
$\mathrm{H}(19)$ & $960(70)$ & $6280(90)$ & $11690(40)$ & $69(15)$ \\
$\mathrm{H}(2)$ & $6180(70)$ & $6060(90)$ & $5930(40)$ & $64(14)$ \\
$\mathrm{H}(15 \mathrm{~A})$ & $4030(60)$ & $8070(90)$ & $8590(40)$ & $61(14)$ \\
$\mathrm{H}(3)$ & $7230(60)$ & $5980(70)$ & $4500(30)$ & $43(10)$ \\
$\mathrm{H}(10 \mathrm{~B})$ & $1340(60)$ & $8180(80)$ & $7660(30)$ & $49(11)$ \\
$\mathrm{H}(20)$ & $2410(80)$ & $3720(120)$ & $11350(50)$ & $85(18)$ \\
$\mathrm{H}(21)$ & $3630(70)$ & $3680(100)$ & $9980(40)$ & $61(13)$ \\
$\mathrm{H}(17)$ & $1960(70)$ & $9210(100)$ & $9490(40)$ & $67(15)$ \\
$\mathrm{H}(9 \mathrm{~A})$ & $-130(60)$ & $4430(90)$ & $7030(30)$ & $58(12)$ \\
$\mathrm{H}(9 \mathrm{~B})$ & $-800(70)$ & $6710(90)$ & $6690(40)$ & $61(13)$ \\
$\mathrm{H}(15 \mathrm{~B})$ & $4840(70)$ & $5830(100)$ & $8850(40)$ & $63(13)$ \\
$\mathrm{H}(5)$ & $2690(60)$ & $5550(80)$ & $3100(30)$ & $52(12)$ \\
& & & & \\
\hline
\end{tabular}




\section{HPLC Spectra}<smiles>N#C[C@]1(Br)CCc2oc(=O)c3ccccc3c2C1=O</smiles>

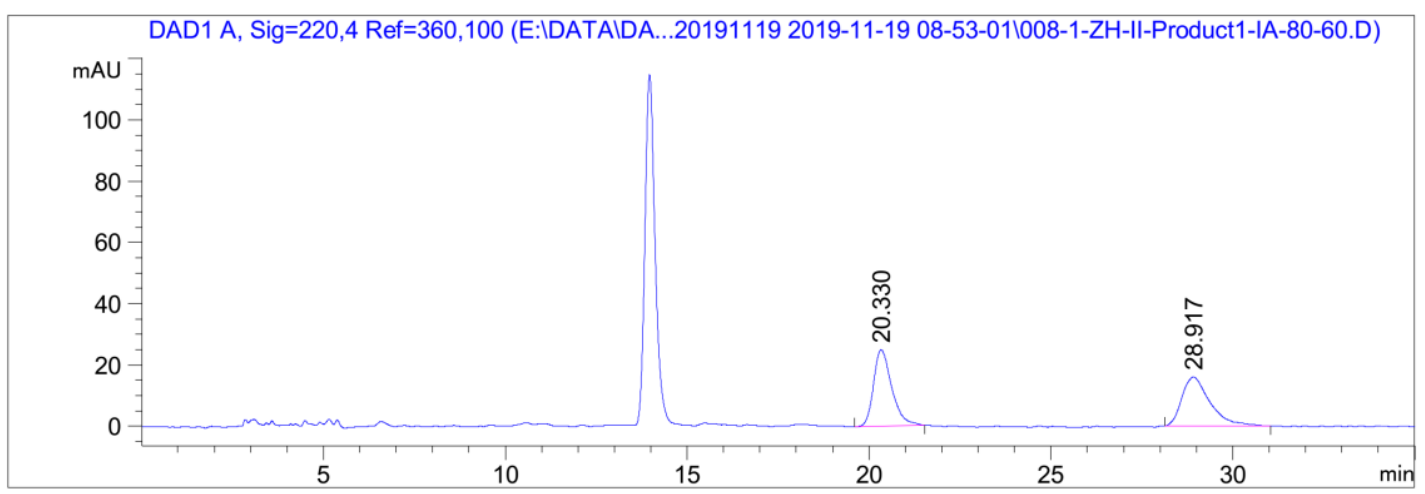

Signal 1: DAD1 A, Sig=220,4 Ref=360,100

\begin{tabular}{|c|c|c|c|c|c|c|}
\hline $\begin{array}{c}\text { Peak } \\
\quad \#\end{array}$ & $\begin{array}{c}\text { RetTime } \\
\text { [min] }\end{array}$ & Type & $\begin{array}{l}\text { Width } \\
\text { [min] }\end{array}$ & $\begin{array}{c}\text { Area } \\
{\left[\mathrm{mAU}^{*} \mathrm{~s}\right]}\end{array}$ & $\begin{array}{l}\text { Height } \\
{[\mathrm{mAU}]}\end{array}$ & $\begin{array}{c}\text { Area } \\
\%\end{array}$ \\
\hline-- & & & & 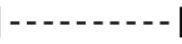 & & \\
\hline 1 & 20.330 & BB & 0.5255 & 865.70575 & 24.90541 & 50.1825 \\
\hline 2 & 28.917 & BB & 0.7916 & 859.40802 & 15.97512 & 49.8175 \\
\hline Total & Is: & & & 1725.11377 & 40.88053 & \\
\hline
\end{tabular}

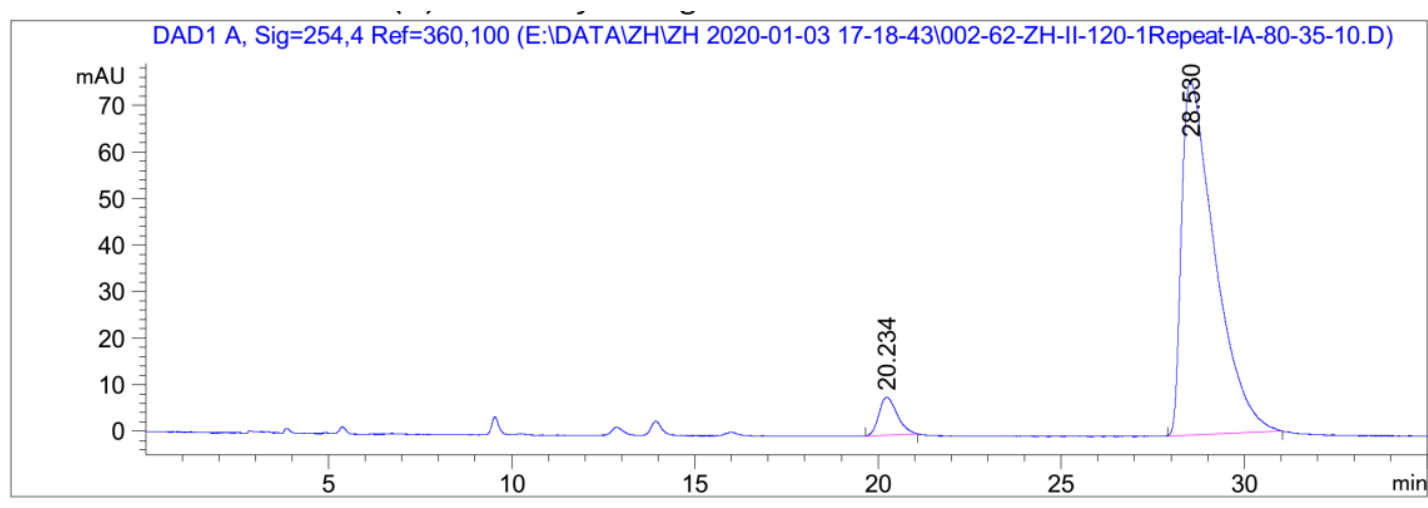

Signal 1: DAD1 A, Sig=254,4 Ref=360,100

\begin{tabular}{|c|c|c|c|c|c|c|}
\hline $\begin{array}{c}\text { Peak } \\
\#\end{array}$ & $\begin{array}{c}\text { RetTime } \\
\text { [min] }\end{array}$ & Type & $\begin{array}{l}\text { Width } \\
\text { [min] }\end{array}$ & $\begin{array}{c}\text { Area } \\
{\left[\mathrm{mAU}^{*} \mathrm{~s}\right]}\end{array}$ & $\begin{array}{l}\text { Height } \\
\text { [mAU] }\end{array}$ & $\begin{array}{c}\text { Area } \\
\%\end{array}$ \\
\hline & & & & |---.- & --1 & --- \\
\hline 1 & 20.234 & BV $R$ & 0.4041 & 282.88770 & 8.19802 & 5.5628 \\
\hline 2 & 28.530 & BB & 0.7389 & 4802.44873 & 76.08106 & 94.4372 \\
\hline Tot & : & & & 5085.33643 & 4.27908 & \\
\hline
\end{tabular}


<smiles>Cc1ccc2c3c(oc(=O)c2c1)CC[C@@]([18O])(Br)C3=O</smiles>

2b

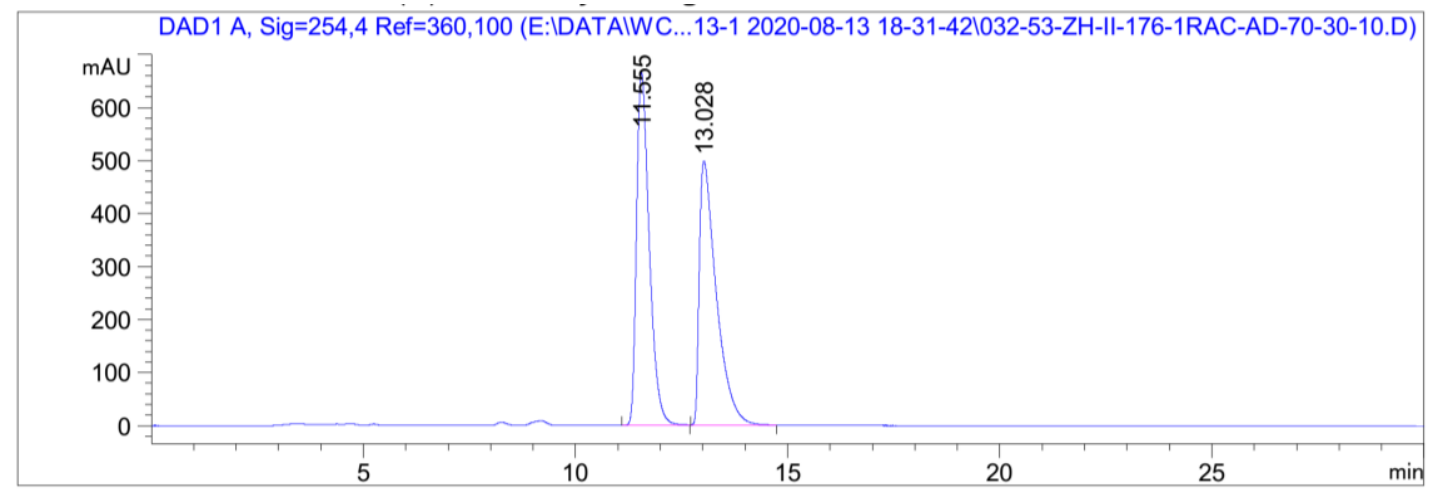

Signal 1: DAD1 A, Sig=254,4 Ref $=360,100$

Peak RetTime Type Width Area Height Area

$\begin{array}{llll}\# & {[\mathrm{~min}]} & {[\mathrm{min}]} & {[\mathrm{mAU} * \mathrm{~s}]}\end{array}$

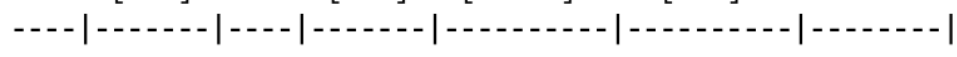

$\begin{array}{lllllll}1 & 11.555 & \text { BB } & 0.3139 & 1.38957 \mathrm{e} 4 & 667.28949 & 50.0151\end{array}$

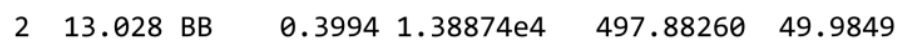

Totals : $\quad 2.77831 \mathrm{e} 4 \quad 1165.17209$

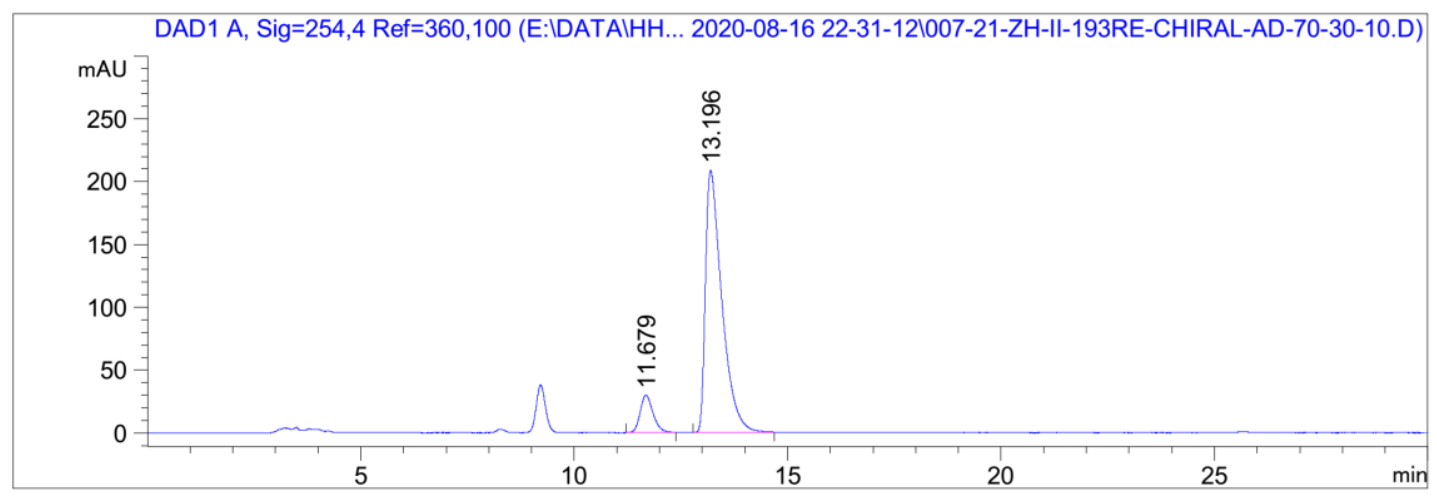

Signal 1: DAD1 A, Sig=254,4 Ref $=360,100$

\begin{tabular}{cccccc}
$\begin{array}{c}\text { Peak RetTime Type } \\
\text { Width }\end{array}$ & $\begin{array}{c}\text { Area } \\
\text { [min] }\end{array}$ & $\begin{array}{c}\text { Height } \\
{[\text { mAU*s }]}\end{array}$ & \multicolumn{1}{c}{$\begin{array}{c}\text { Area } \\
\text { [mAU] }\end{array}$} & \multicolumn{1}{c}{$\%$} \\
\hline 1 & 11.679 BB & 0.2598 & 622.93201 & 29.69354 & 10.0537 \\
2 & 13.196 BB & 0.3764 & 5573.14404 & 208.44884 & 89.9463
\end{tabular}

Totals :

$6196.07605 \quad 238.14238$ 
<smiles>Cc1ccc2c(=O)oc3c(c2c1)C(=O)C(C)(Br)CC3</smiles>

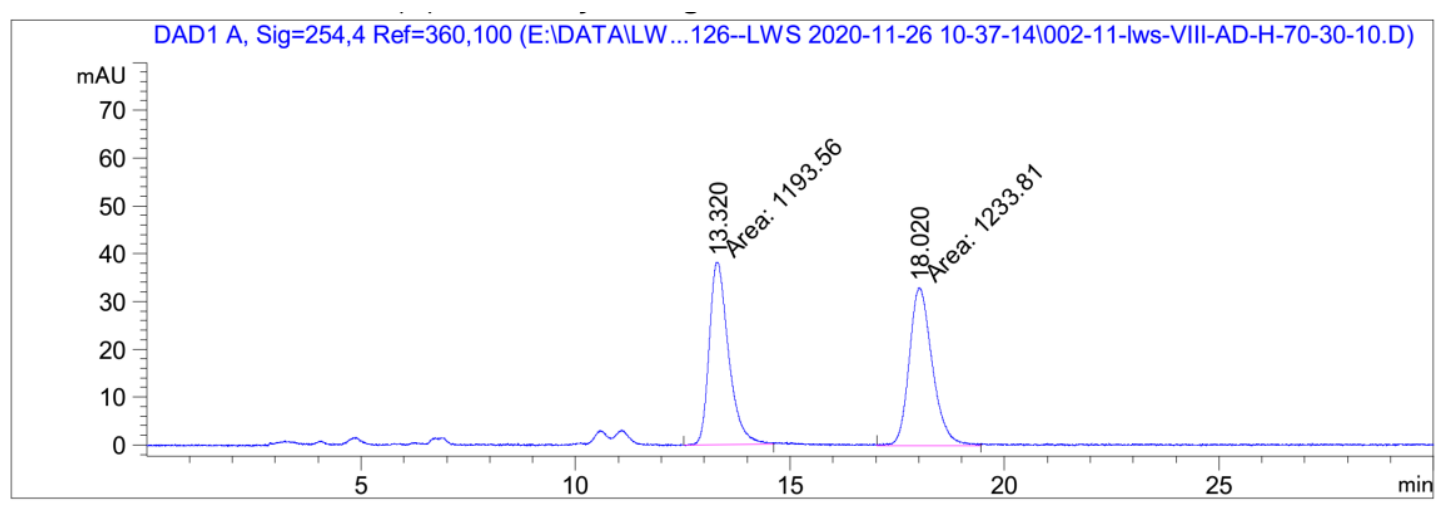

Signal 1: DAD1 A, Sig $=254,4$ Ref $=360,100$

\begin{tabular}{|c|c|c|c|c|c|c|}
\hline $\begin{array}{c}\text { Peak } \\
\quad \#\end{array}$ & $\begin{array}{c}\text { RetTime } \\
\text { [min] }\end{array}$ & Type & $\begin{array}{l}\text { Width } \\
\text { [min] }\end{array}$ & $\begin{array}{c}\text { Area } \\
{\left[\mathrm{mAU}^{*} \mathrm{~s}\right]}\end{array}$ & $\begin{array}{l}\text { Height } \\
{[\mathrm{mAU}]}\end{array}$ & $\begin{array}{c}\text { Area } \\
\%\end{array}$ \\
\hline---1 & | $-\ldots$ & -7 & - & $\mid-\ldots$ & -- &.- \\
\hline 1 & 13.320 & MM & 0.5218 & 1193.55518 & 38.12642 & 49.1709 \\
\hline 2 & 18.020 & MM & 0.6230 & 1233.80676 & 33.00644 & 50.8291 \\
\hline Tota] & $15:$ & & & 2427.36194 & 71.13285 & \\
\hline
\end{tabular}

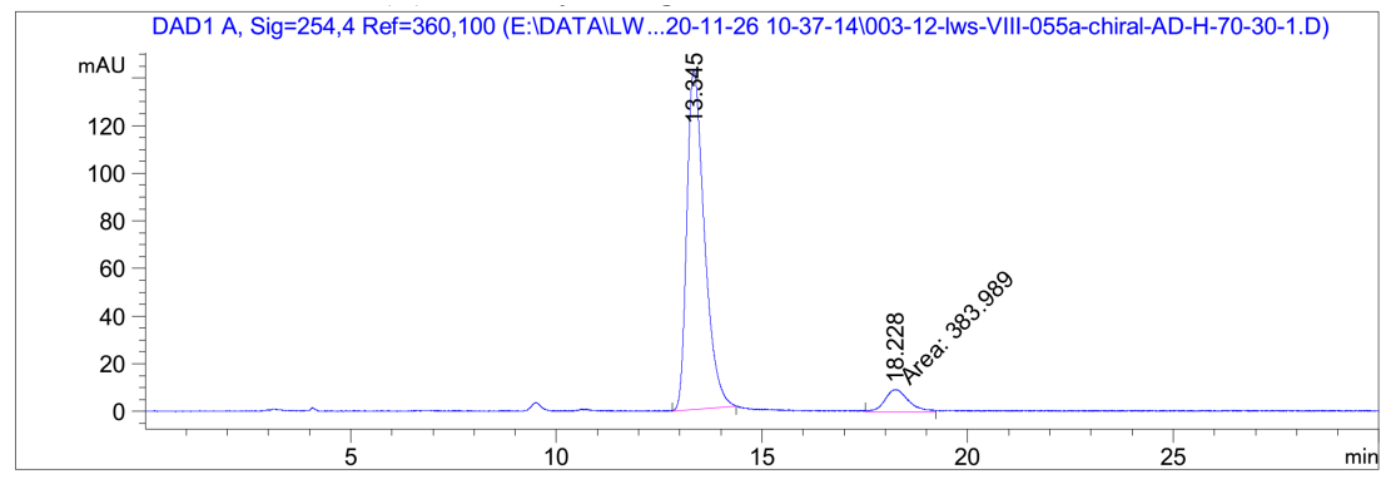

Signal 1: DAD1 A, Sig=254,4 Ref $=360,100$

\begin{tabular}{|c|c|c|c|c|c|}
\hline $\begin{array}{c}\text { Peak } \\
\#\end{array}$ & $\begin{array}{l}\text { RetTime Type } \\
\text { [min] }\end{array}$ & $\begin{array}{l}\text { Width } \\
\text { [min] }\end{array}$ & $\begin{array}{c}\text { Area } \\
{\left[\mathrm{mAU}^{*} \mathrm{~s}\right]}\end{array}$ & $\begin{array}{l}\text { Height } \\
{[\mathrm{mAU}]}\end{array}$ & $\begin{array}{c}\text { Area } \\
\%\end{array}$ \\
\hline-- & 1 & 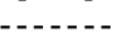 & - & - & 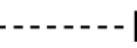 \\
\hline 1 & $13.345 \mathrm{VV} \mathrm{R}$ & 0.3498 & 4237.77686 & 142.78053 & 91.6917 \\
\hline 2 & $18.228 \mathrm{MM}$ & 0.6746 & 383.98944 & 9.48669 & 8.3083 \\
\hline Tota] & ls: & & 4621.76630 & 152.26722 & \\
\hline
\end{tabular}


<smiles>COc1ccc2c3c(oc(=O)c2c1)CC[C@](Cc1ccccc1)(C(=O)[O-])C3=O</smiles>

2d

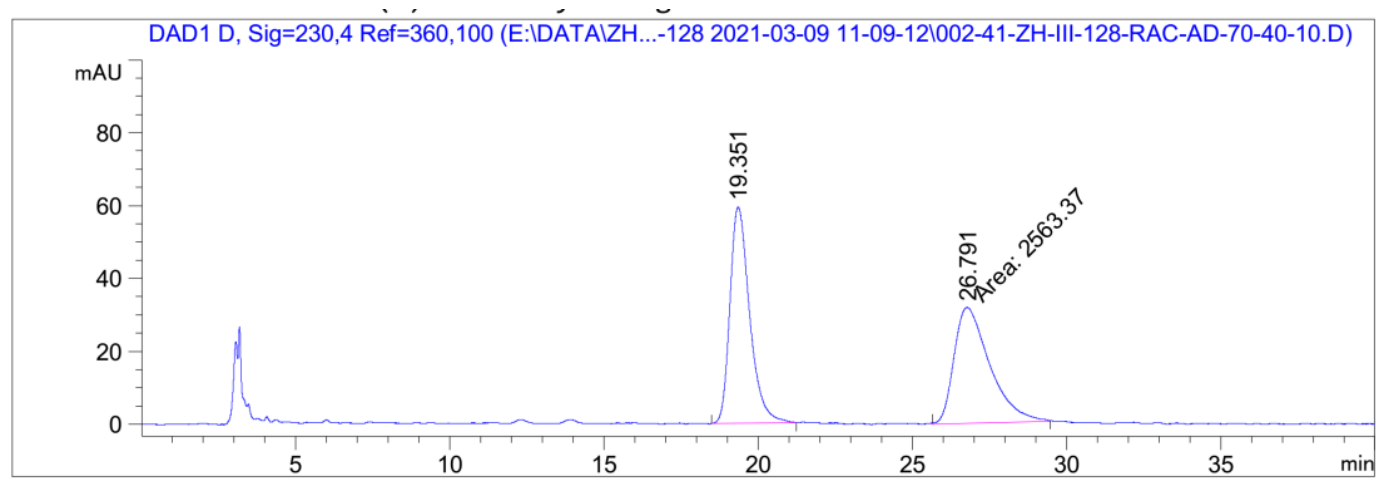

Signal 1: DAD1 D, Sig=230,4 Ref=360,100

\begin{tabular}{|c|c|c|c|c|c|c|}
\hline $\begin{array}{c}\text { Peak } \\
\#\end{array}$ & $\begin{array}{c}\text { RetTime } \\
\text { [min] }\end{array}$ & Type & $\begin{array}{l}\text { Width } \\
\text { [min] }\end{array}$ & $\begin{array}{c}\text { Area } \\
{\left[\mathrm{mAU}^{*} \mathrm{~s}\right]}\end{array}$ & $\begin{array}{l}\text { Height } \\
{[\mathrm{mAU}]}\end{array}$ & $\begin{array}{c}\text { Area } \\
\%\end{array}$ \\
\hline & & & & & & \\
\hline 1 & 19. & & & 2637.82715 & 377 & 50.7158 \\
\hline 2 & 26. & & 8 & 2563.36719 & 9336 & 49.2842 \\
\hline
\end{tabular}

Totals :

$5201.19434 \quad 91.22713$

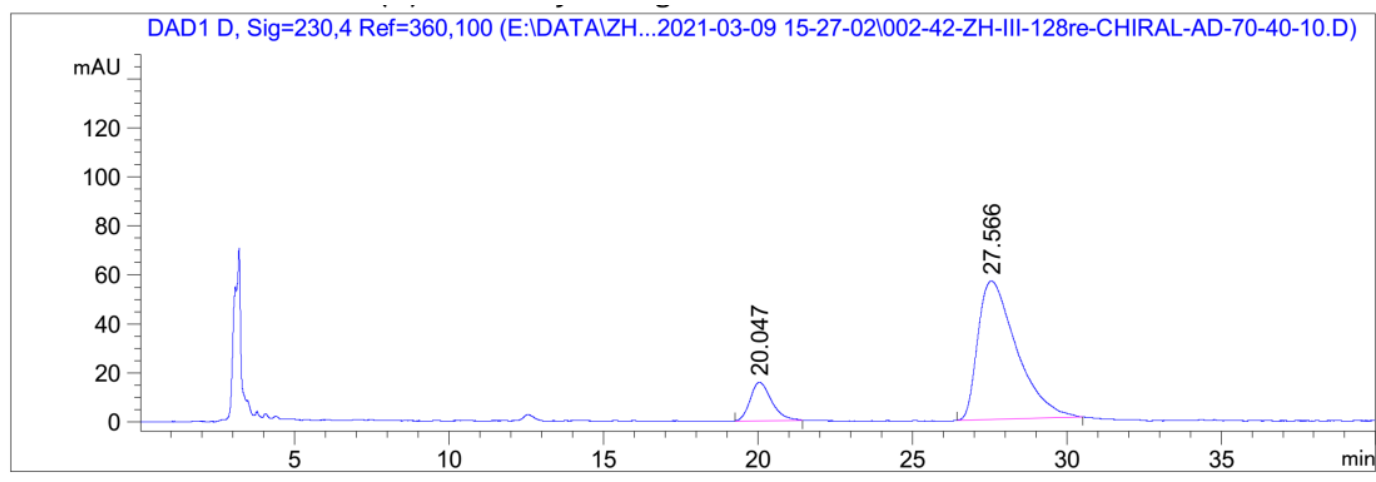

Signal 1: DAD1 D, Sig=230,4 Ref $=360,100$

\begin{tabular}{|c|c|c|c|c|c|c|}
\hline $\begin{array}{c}\text { Peak } \\
\#\end{array}$ & $\begin{array}{c}\text { RetTime } \\
\text { [min] }\end{array}$ & Type & $\begin{array}{l}\text { Width } \\
\text { [min] }\end{array}$ & $\begin{array}{c}\text { Area } \\
{\left[\mathrm{mAU}^{*} \mathrm{~s}\right]}\end{array}$ & $\begin{array}{l}\text { Height } \\
{[\mathrm{mAU}]}\end{array}$ & $\begin{array}{c}\text { Area } \\
\%\end{array}$ \\
\hline & . & & & ---1 & -1 & -- \\
\hline 1 & 20.047 & BB & 0.5603 & 751.85687 & 15.71179 & 13.2367 \\
\hline 2 & 27.566 & BB & 1.0212 & 4928.21924 & 56.52215 & 86.7633 \\
\hline Tota] & Is: & & & 5680.07611 & 72.23394 & \\
\hline
\end{tabular}


<smiles>N#C[C@@]1(Br)CCc2oc(=O)c3cc(F)ccc3c2C1=O</smiles>

2e

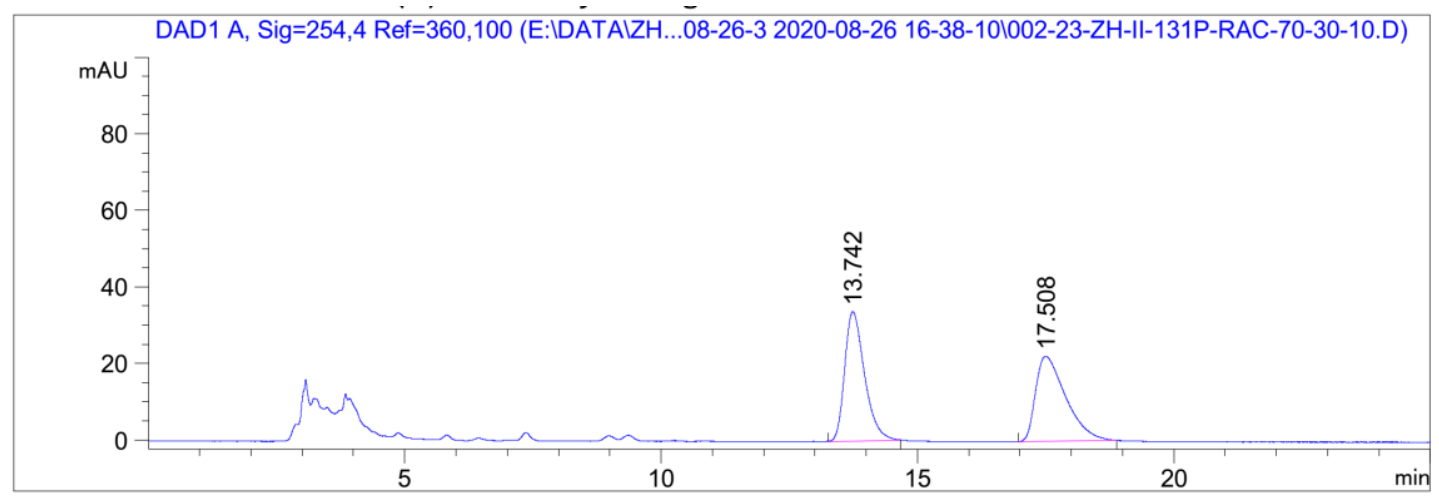

Signal 1: DAD1 A, Sig=254,4 Ref $=360,100$

\begin{tabular}{|c|c|c|c|c|c|c|}
\hline $\begin{array}{c}\text { Peak } \\
\#\end{array}$ & $\begin{array}{c}\text { RetTime } \\
\text { [min] }\end{array}$ & Type & $\begin{array}{l}\text { Width } \\
\text { [min] }\end{array}$ & $\begin{array}{c}\text { Area } \\
{\left[\mathrm{mAU}{ }^{*} \mathrm{~s}\right]}\end{array}$ & $\begin{array}{l}\text { Height } \\
{[\mathrm{mAU}]}\end{array}$ & $\begin{array}{c}\text { Area } \\
\%\end{array}$ \\
\hline--- & | - - - & & 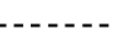 & | & | & | \\
\hline 1 & 13.742 & BB & 0.3283 & 906.71637 & 33.76957 & 49.9862 \\
\hline 2 & 17.508 & BB & 0.4808 & 907.21716 & 22.22340 & 50.0138 \\
\hline Tot & : & & & 1813.93353 & 55.99298 & \\
\hline
\end{tabular}

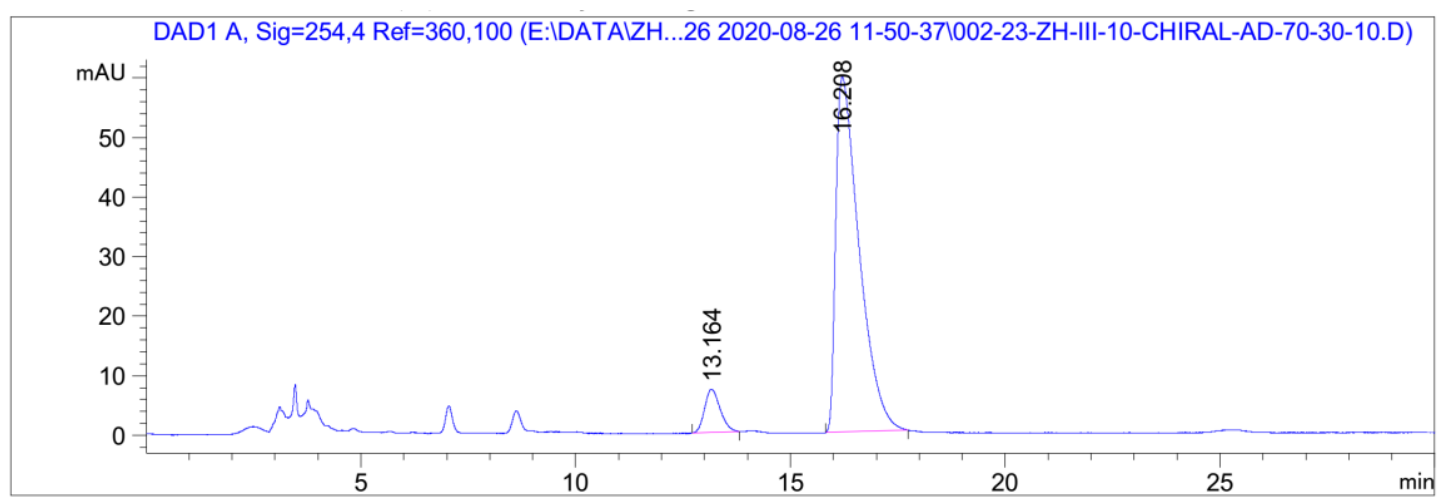

Signal 1: DAD1 A, Sig=254,4 Ref $=360,100$

\begin{tabular}{cccccc}
$\begin{array}{c}\text { Peak RetTime Type Width } \\
\text { \# }[\mathrm{min}]\end{array}$ & $\begin{array}{c}\text { Area } \\
{[\mathrm{min}]}\end{array}$ & $\begin{array}{l}\text { Height } \\
{\left[\mathrm{mAU}{ }^{*} \mathrm{~s}\right]}\end{array}$ & $\begin{array}{c}\text { Area } \\
{[\mathrm{mAU}]}\end{array}$ & $\%$ \\
\hline 1 & 13.164 BB & 0.2968 & 183.41925 & 7.29546 & 7.5134 \\
2 & 16.208 BB & 0.4448 & 2257.81592 & 59.61753 & 92.4866
\end{tabular}

Totals :

$2441.23517 \quad 66.91299$ 
<smiles>N#CC1(Br)CCc2oc(=O)c3cc(Cl)ccc3c2C1=O</smiles>

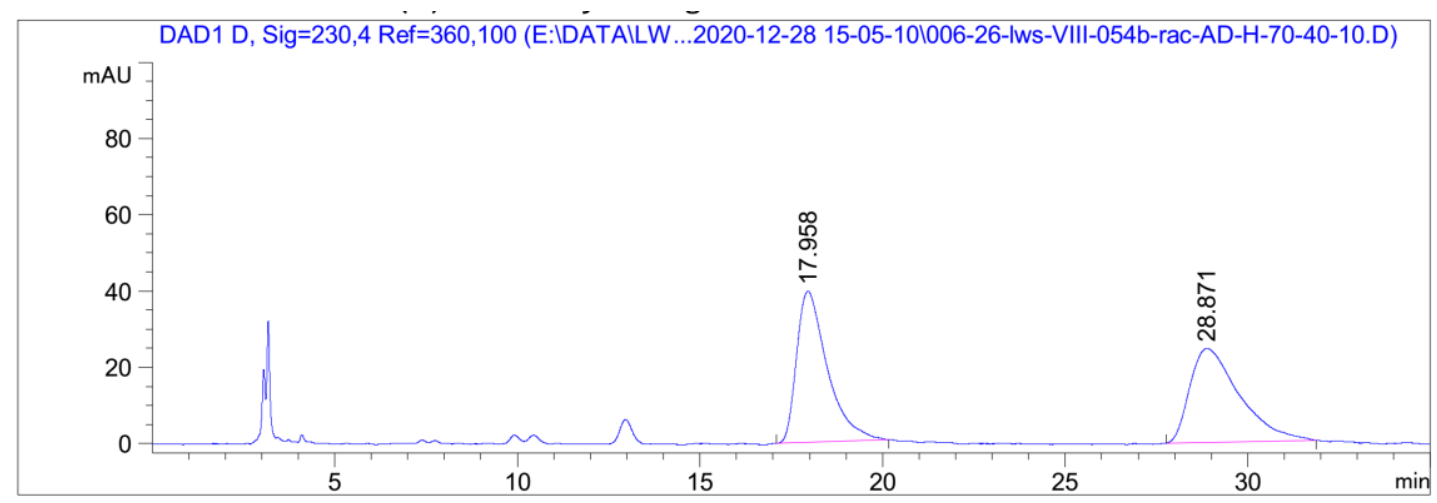

Signal 1: DAD1 D, Sig=230,4 Ref=360,100

\begin{tabular}{|c|c|c|c|c|c|c|}
\hline $\begin{array}{c}\text { Peak } \\
\quad \#\end{array}$ & $\begin{array}{c}\text { RetTime } \\
\text { [min] }\end{array}$ & Type & $\begin{array}{l}\text { Width } \\
\text { [min] }\end{array}$ & $\begin{array}{c}\text { Area } \\
{\left[\mathrm{mAU}^{*} \mathrm{~s}\right]}\end{array}$ & $\begin{array}{l}\text { Height } \\
\text { [mAU] }\end{array}$ & $\begin{array}{c}\text { Area } \\
\%\end{array}$ \\
\hline & & & & -2 & & - \\
\hline 1 & 17.958 & BB & 0.6856 & 2317.38574 & 39.63618 & 49.9901 \\
\hline 2 & 28.871 & BB & 1.0998 & 2318.30371 & 24.67027 & 50.0099 \\
\hline$\Gamma 0$ & . & & & 4635.68945 & 4.30645 & \\
\hline
\end{tabular}

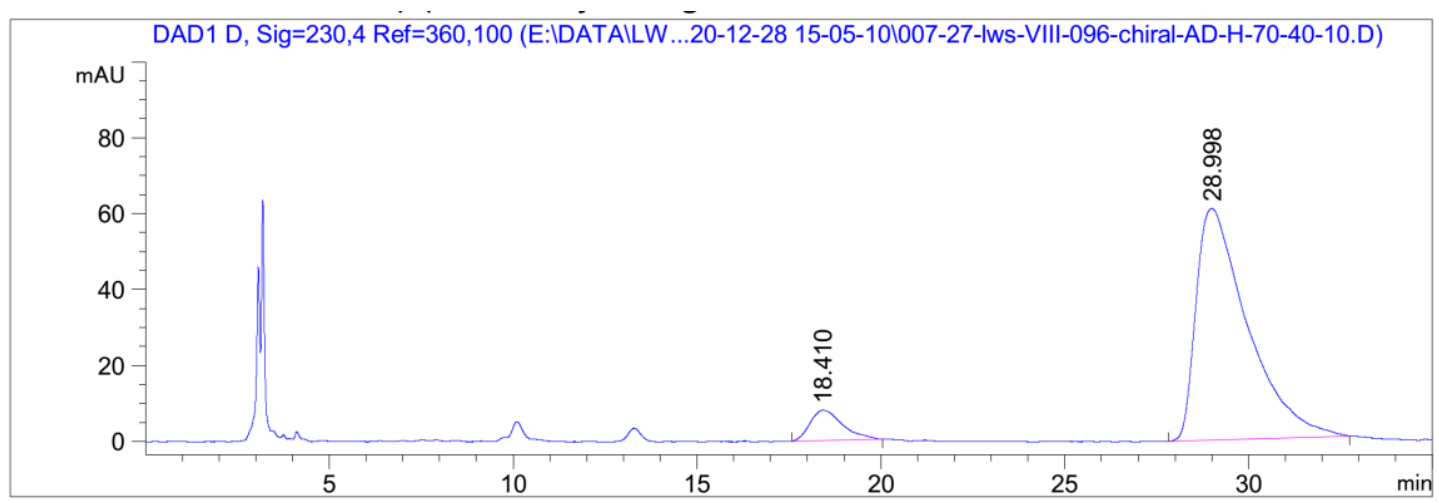

Signal 1: DAD1 D, Sig=230,4 Ref=360,100

\begin{tabular}{|c|c|c|c|c|c|c|}
\hline $\begin{array}{c}\text { Peak } \\
\quad \#\end{array}$ & $\begin{array}{c}\text { RetTime } \\
\text { [min] }\end{array}$ & Type & $\begin{array}{l}\text { Width } \\
\text { [min] }\end{array}$ & $\begin{array}{c}\text { Area } \\
{\left[\mathrm{mAU}^{*} \mathrm{~s}\right]}\end{array}$ & $\begin{array}{l}\text { Height } \\
{[\mathrm{mAU}]}\end{array}$ & $\begin{array}{c}\text { Area } \\
\%\end{array}$ \\
\hline-- & & & -- & |--- & -- & --- \\
\hline 1 & 18.410 & $\mathrm{BB}$ & 0.7205 & 492.04684 & 8.00025 & 7.6558 \\
\hline 2 & 28.998 & BB & 1.1377 & 5935.08789 & 61.04960 & 92.3442 \\
\hline - & : & & & 6427.13474 & 985 & \\
\hline
\end{tabular}


<smiles>N#C[C@@]1(Br)CCc2oc(=O)c3cc(C(F)(F)F)ccc3c2C1=O</smiles>

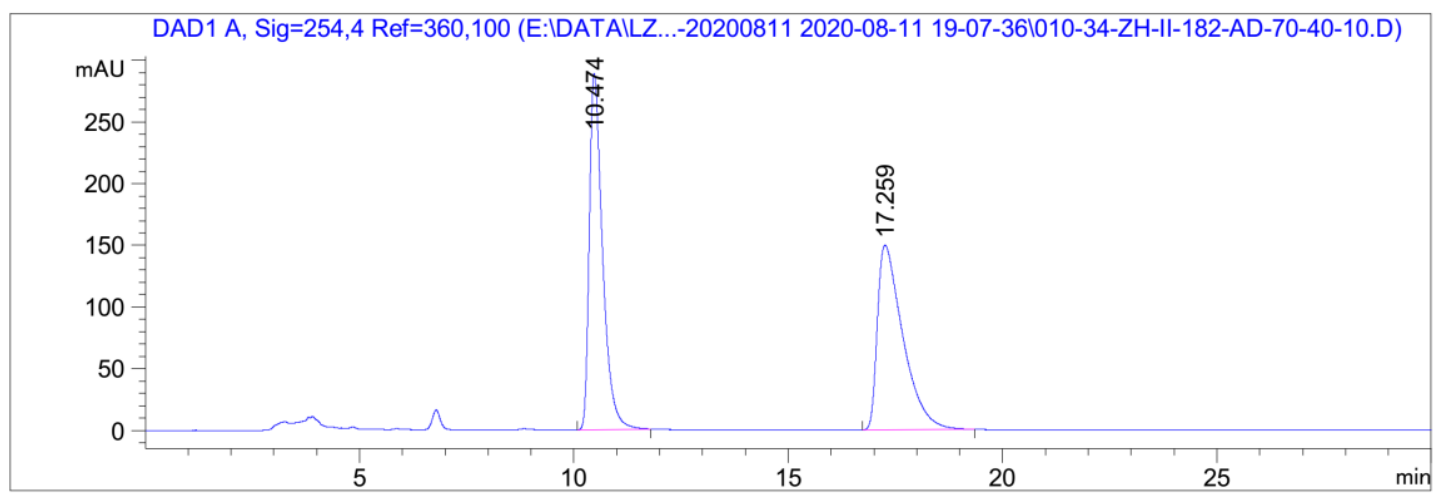

Signal 1: DAD1 A, Sig=254,4 Ref=360,100

\begin{tabular}{|c|c|c|c|c|c|c|}
\hline $\begin{array}{c}\text { Peak } \\
\quad \#\end{array}$ & $\begin{array}{c}\text { RetTime } \\
\text { [min] }\end{array}$ & Type & $\begin{array}{l}\text { Width } \\
\text { [min] }\end{array}$ & $\begin{array}{c}\text { Area } \\
{\left[\mathrm{mAU}^{*} \mathrm{~s}\right]}\end{array}$ & $\begin{array}{l}\text { Height } \\
\text { [mAU] }\end{array}$ & $\begin{array}{c}\text { Area } \\
\%\end{array}$ \\
\hline & & & & -- & & \\
\hline 1 & & 3B & & 6194.02246 & 288.60669 & 50.0213 \\
\hline 2 & 17.259 & BB & 0.5488 & 6188.74707 & 149.86758 & 49.9787 \\
\hline
\end{tabular}

Totals : $\quad 1.23828 \mathrm{e} 4 \quad 438.47427$

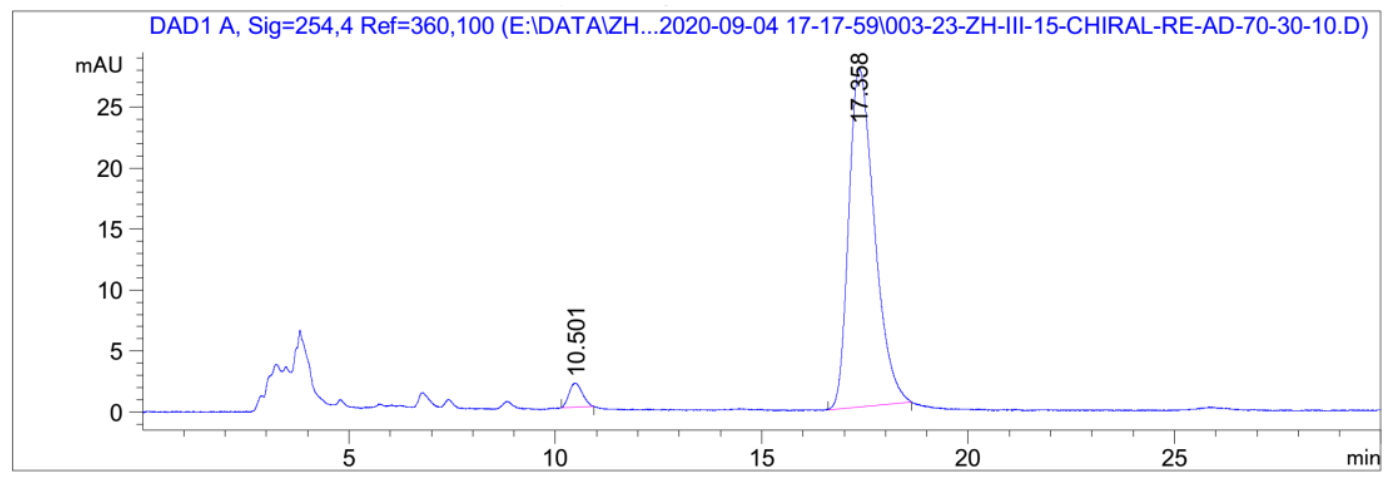

Signal 1: DAD1 A, Sig=254,4 Ref $=360,100$

\begin{tabular}{|c|c|c|c|c|c|c|}
\hline $\begin{array}{c}\text { Peak } \\
\#\end{array}$ & $\begin{array}{c}\text { RetTime } \\
\text { [min] }\end{array}$ & Type & $\begin{array}{l}\text { Width } \\
\text { [min] }\end{array}$ & $\begin{array}{c}\text { Area } \\
{\left[\mathrm{mAU}^{*} \mathrm{~s}\right]}\end{array}$ & $\begin{array}{l}\text { Height } \\
{[\mathrm{mAU}]}\end{array}$ & $\begin{array}{c}\text { Area } \\
\%\end{array}$ \\
\hline & & & & - & & -- \\
\hline 1 & 10.501 & BB & 2697 & 45.26406 & 2.00234 & 3.6305 \\
\hline 2 & 17.358 & BB & .5077 & 1201.52039 & 27.69333 & 96.3695 \\
\hline
\end{tabular}

Totals :

$1246.78445 \quad 29.69567$ 
<smiles>CC(=O)c1ccc2c3c(oc(=O)c2c1)CC[C@@](C#N)(C(C)=O)C3=O</smiles>

2h

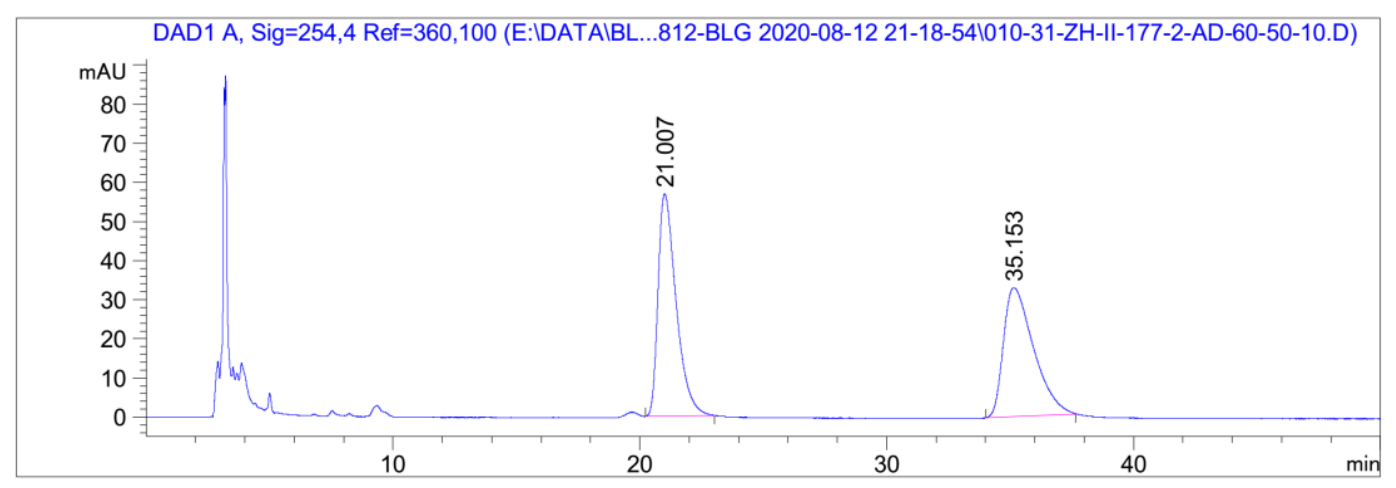

Signal 1: DAD1 A, Sig=254,4 Ref=360,100
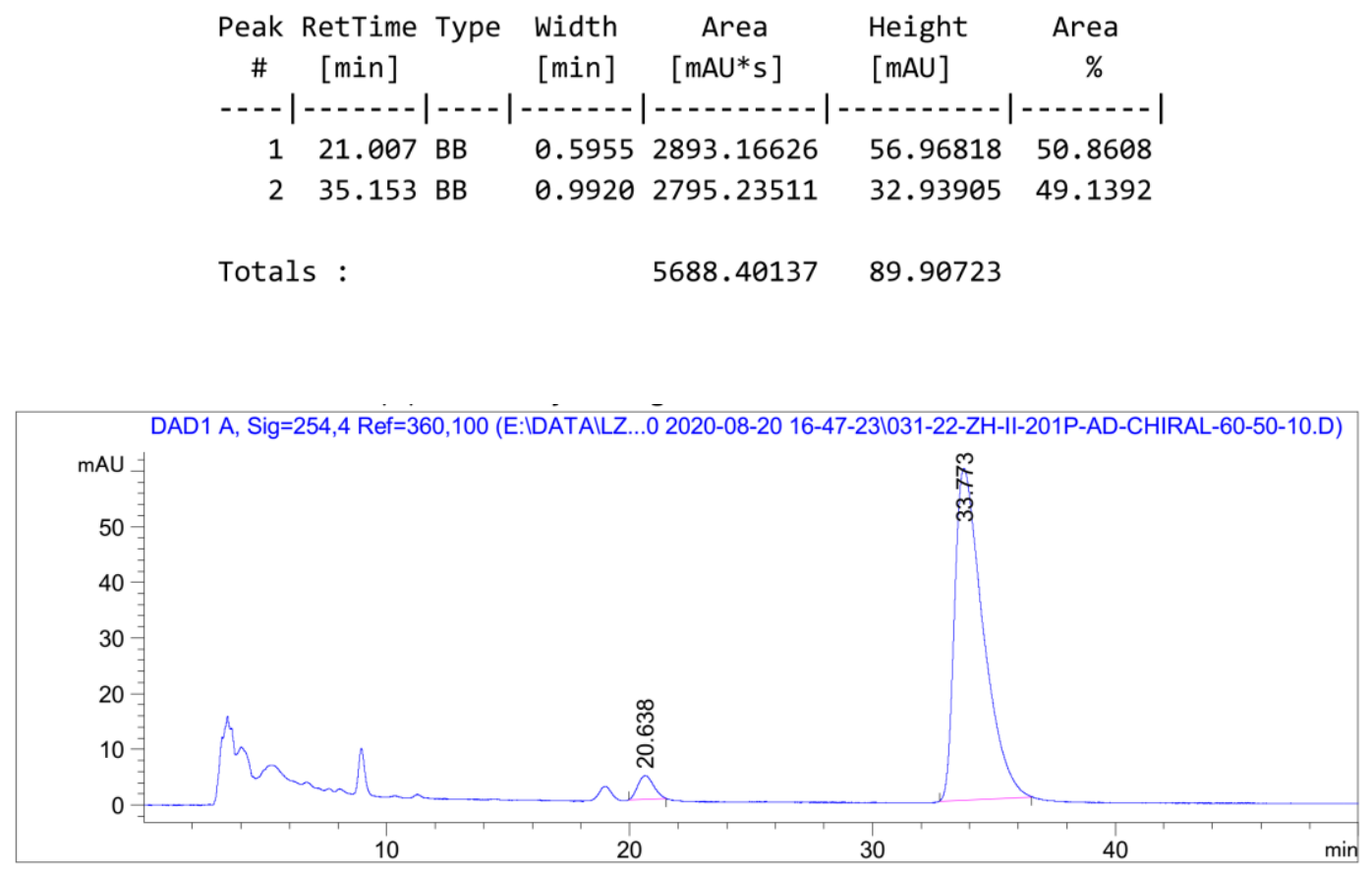

Signal 1: DAD1 A, Sig=254,4 Ref=360,100

\begin{tabular}{|c|c|c|c|c|c|c|}
\hline $\begin{array}{c}\text { Peak } \\
\#\end{array}$ & $\begin{array}{c}\text { RetTime } \\
\text { [min] }\end{array}$ & Type & $\begin{array}{l}\text { Width } \\
\text { [min] }\end{array}$ & $\begin{array}{c}\text { Area } \\
{\left[\mathrm{mAU}^{*} \mathrm{~s}\right]}\end{array}$ & $\begin{array}{l}\text { Height } \\
{[\mathrm{mAU}]}\end{array}$ & $\begin{array}{c}\text { Area } \\
\%\end{array}$ \\
\hline & & & & & & \\
\hline 1 & 20 & $\mathrm{R}$ & 0 . & 190.63544 & 4.25689 & 319 \\
\hline ? & 73 & B & 0.9532 & 4850.11768 & 59.58224 & 181 \\
\hline
\end{tabular}

Totals :

$5040.75311 \quad 63.83913$ 
<smiles>N#CC1(Br)CCc2oc(=O)c3c(c2C1=O)CCCC3</smiles>

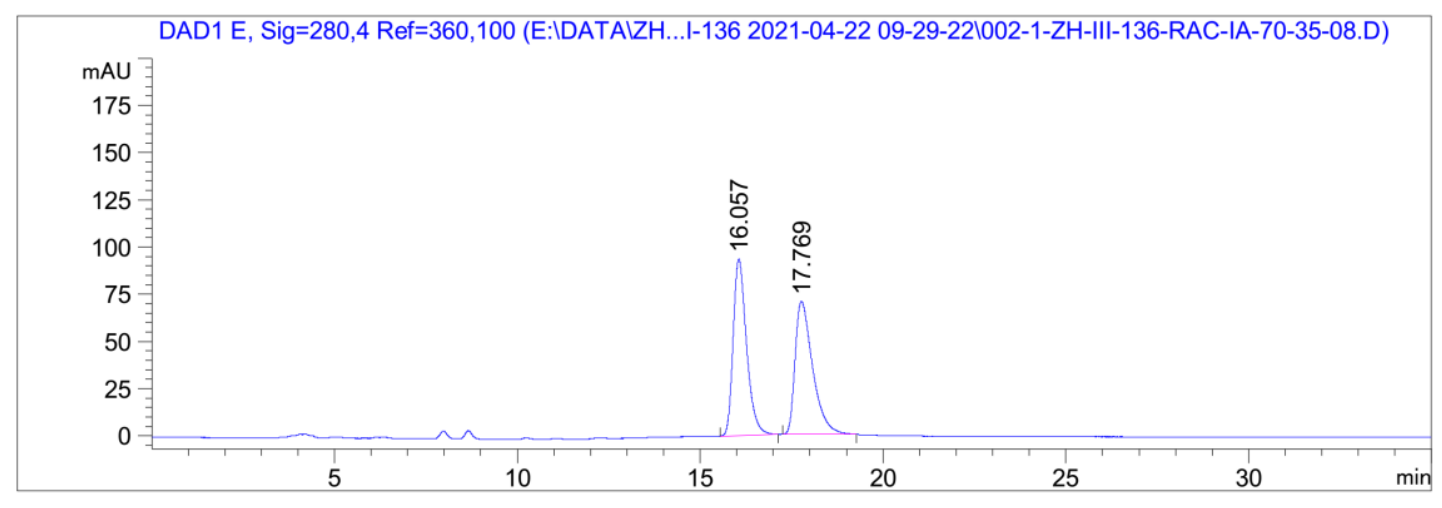

Signal 1: DAD1 E, Sig $=280,4$ Ref $=360,100$

\begin{tabular}{|c|c|c|c|c|c|c|}
\hline $\begin{array}{c}\text { Peak } \\
\#\end{array}$ & $\begin{array}{c}\text { RetTime } \\
\text { [min] }\end{array}$ & Type & $\begin{array}{l}\text { Width } \\
\text { [min] }\end{array}$ & $\begin{array}{c}\text { Area } \\
{\left[\mathrm{mAU}^{*} \mathrm{~s}\right]}\end{array}$ & $\begin{array}{l}\text { Height } \\
{[\mathrm{mAU}]}\end{array}$ & $\begin{array}{c}\text { Area } \\
\%\end{array}$ \\
\hline 1 & -- & & & | - - - & . & - \\
\hline 1 & 16.057 & BB & 0.3579 & 2378.92529 & 93.47517 & 50.2785 \\
\hline 2 & 17.769 & BB & 0.4079 & 2352.57251 & 70.48984 & 49.7215 \\
\hline t & : & & & 4731.49780 & 163.96500 & \\
\hline
\end{tabular}

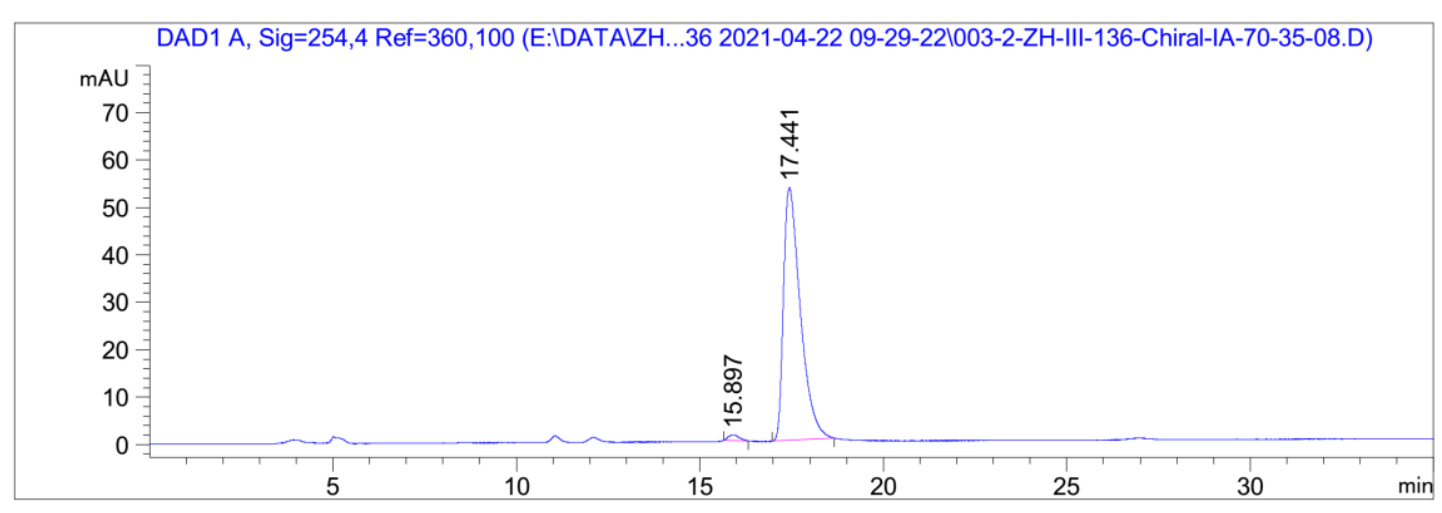

Signal 1: DAD1 A, Sig=254,4 Ref=360,100

\begin{tabular}{cccccc}
$\begin{array}{c}\text { Peak RetTime Type } \\
\text { Width } \\
\text { [min] }\end{array}$ & $\begin{array}{c}\text { Area } \\
{[\mathrm{min}]}\end{array}\left[\begin{array}{l}\text { HAU*s] } \\
{[\mathrm{mAU}]}\end{array}\right.$ & $\begin{array}{c}\text { Area } \\
\%\end{array}$ \\
\hline 1 & 15.897 BB & 0.2410 & 24.56682 & 1.20405 & 1.4209 \\
2 & 17.441 BB & 0.3883 & 1704.37231 & 53.34074 & 98.5791
\end{tabular}

Totals :

$1728.93913 \quad 54.54479$ 
<smiles>CC1(C)CCc2oc(=O)c3ccccc3c2C1=O</smiles>

2j

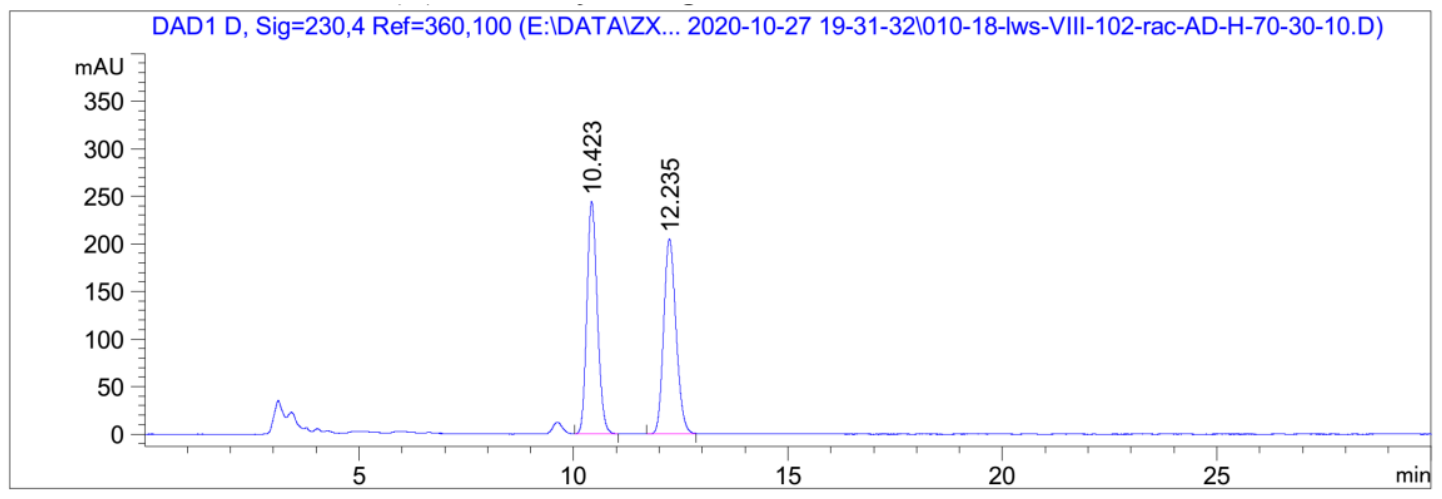

Signal 1: DAD1 D, Sig=230,4 Ref $=360,100$

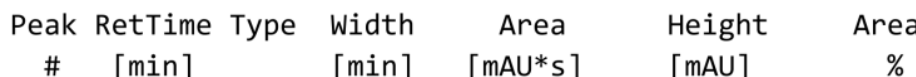

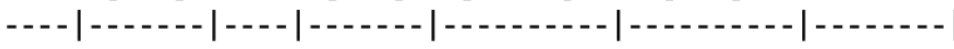

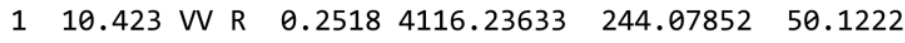

$\begin{array}{lllllllll}2 & 12.235 & V B & R & 0.2921 & 4096.17090 & 205.03471 & 49.8778\end{array}$

Totals :

$8212.40723 \quad 449.11324$

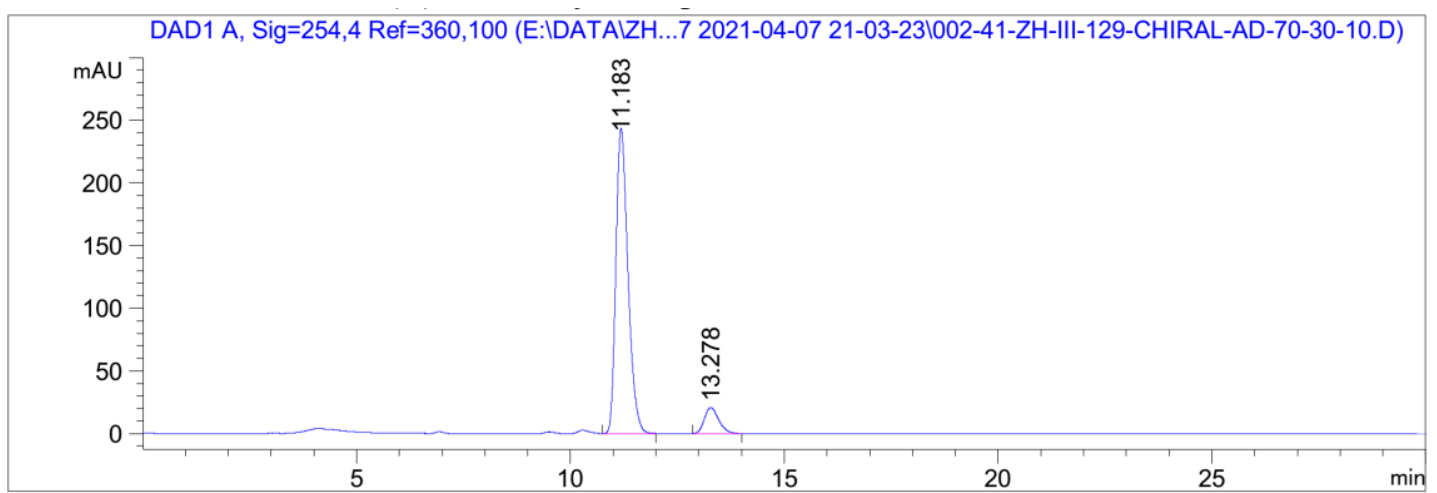

Signal 1: DAD1 A, Sig=254,4 Ref $=360,100$

Peak RetTime Type Width Area Height Area

$\begin{array}{llll}\# & {[\mathrm{~min}]} & {[\mathrm{min}]} & {[\mathrm{mAU} * \mathrm{~s}]}\end{array}$

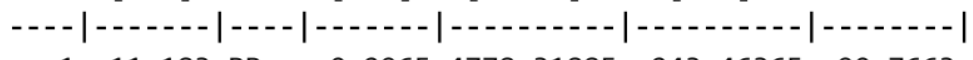

$\begin{array}{lllllll}1 & 11.183 & \text { BB } & 0.2965 & 4778.31885 & 243.46365 & 90.7663\end{array}$

$\begin{array}{lllllll}2 & 13.278 & \text { BB } & 0.2774 & 486.10080 & 20.89694 & 9.2337\end{array}$

Totals : $\quad 5264.41965264 .36060$ 
<smiles>N#CC1(c2ccccc2)CCc2oc(=O)c3ccccc3c2C1=O</smiles>

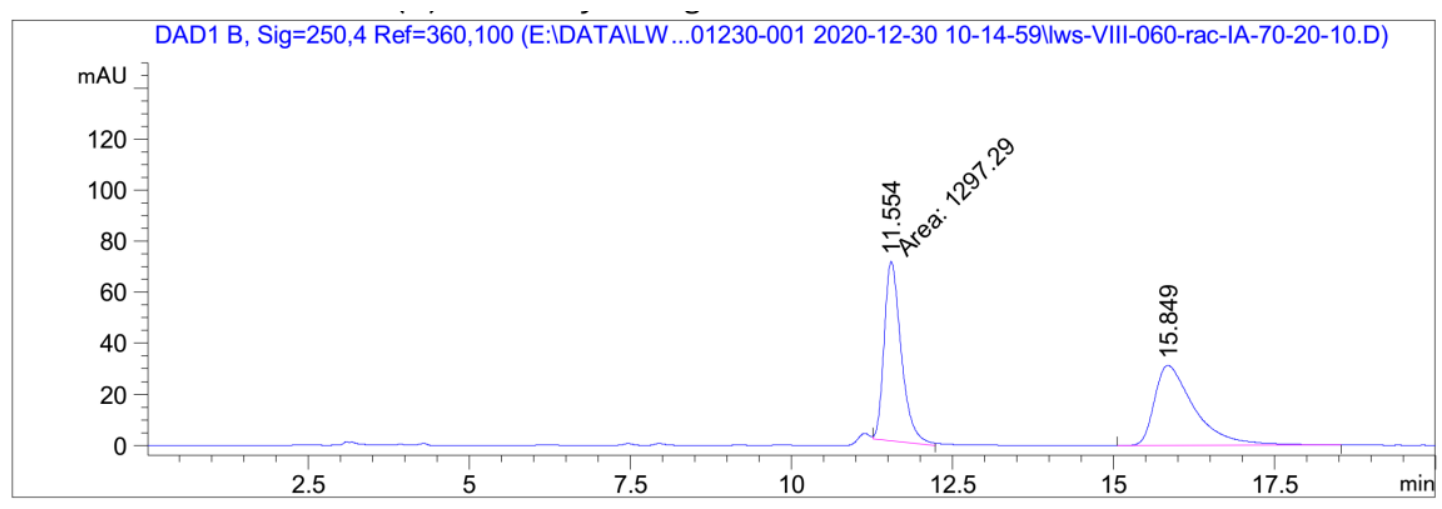

Signal 1: DAD1 B, Sig=250,4 Ref $=360,100$

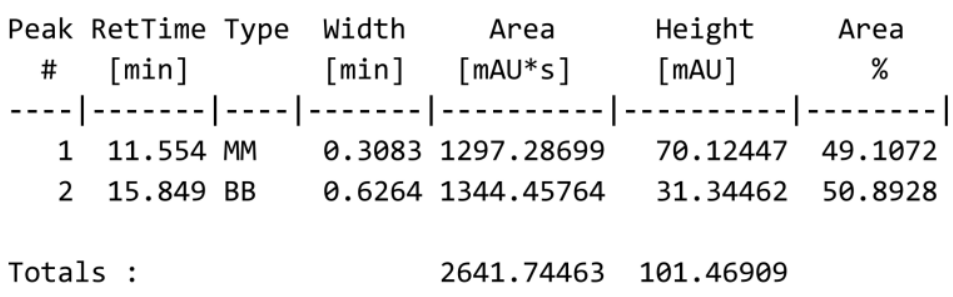

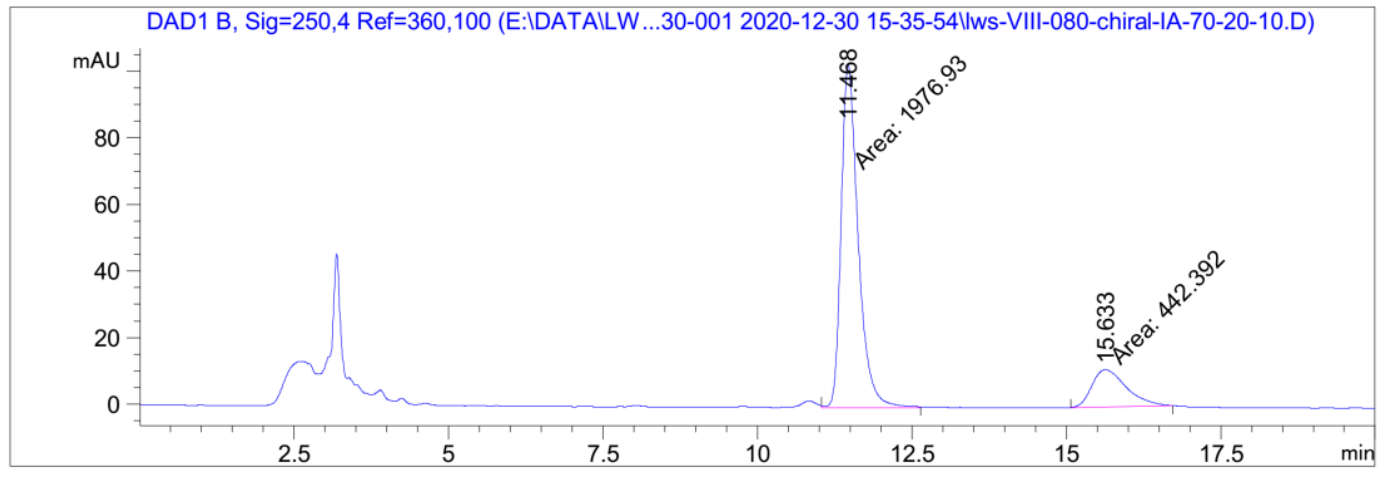

Signal 1: DAD1 B, Sig=250,4 Ref $=360,100$

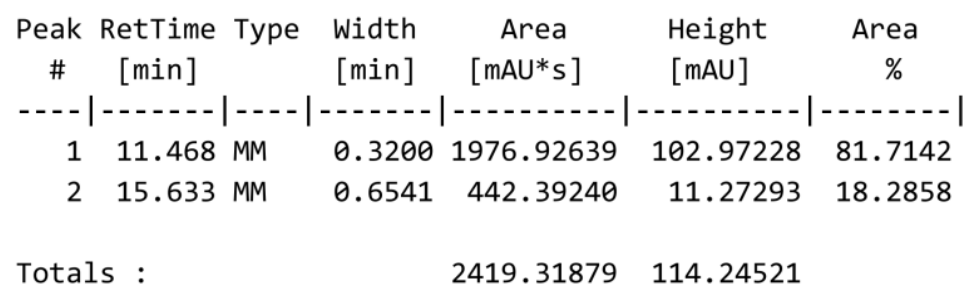



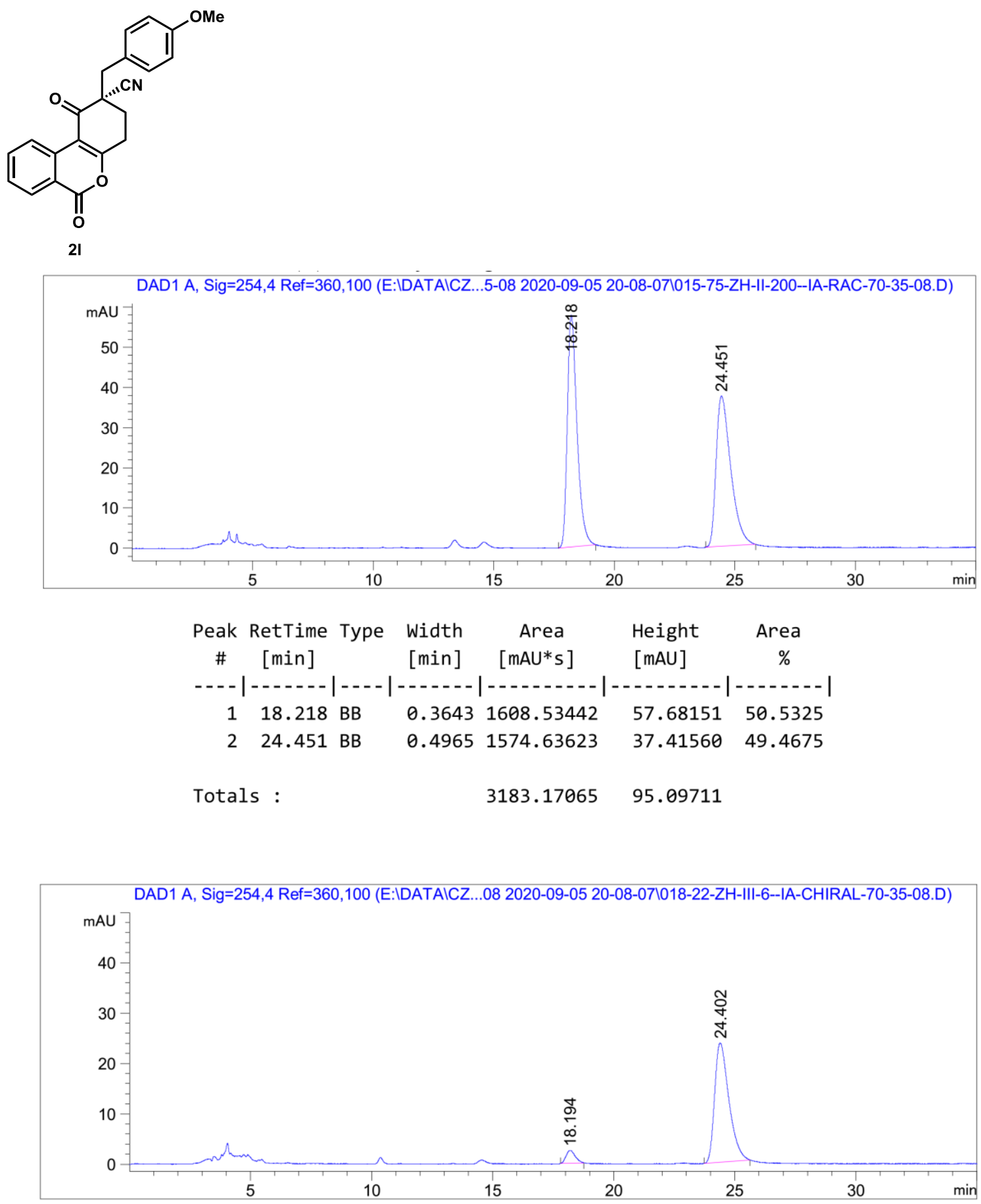

Signal 1: DAD1 A, Sig=254,4 Ref $=360,100$

\begin{tabular}{|c|c|c|c|c|c|}
\hline $\begin{array}{c}\text { Peak } \\
\text { \# }\end{array}$ & $\begin{array}{l}\text { RetTime Type } \\
\text { [min] }\end{array}$ & $\begin{array}{l}\text { Width } \\
{[\mathrm{min}]}\end{array}$ & $\begin{array}{c}\text { Area } \\
{\left[\mathrm{mAU}^{*} \mathrm{~s}\right]}\end{array}$ & $\begin{array}{l}\text { Height } \\
\text { [mAU] }\end{array}$ & $\begin{array}{c}\text { Area } \\
\%\end{array}$ \\
\hline . & - & . & $-\ldots$ & $-\ldots$ & --- - - \\
\hline 1 & $18.194 \mathrm{BB}$ & 0.3093 & 66.88452 & 2.55157 & 6.4547 \\
\hline 2 & 24.402 BB & 0.4803 & 969.33240 & 23.72064 & 93.5453 \\
\hline ot & . & & 036.21692 & 26.27221 & \\
\hline
\end{tabular}



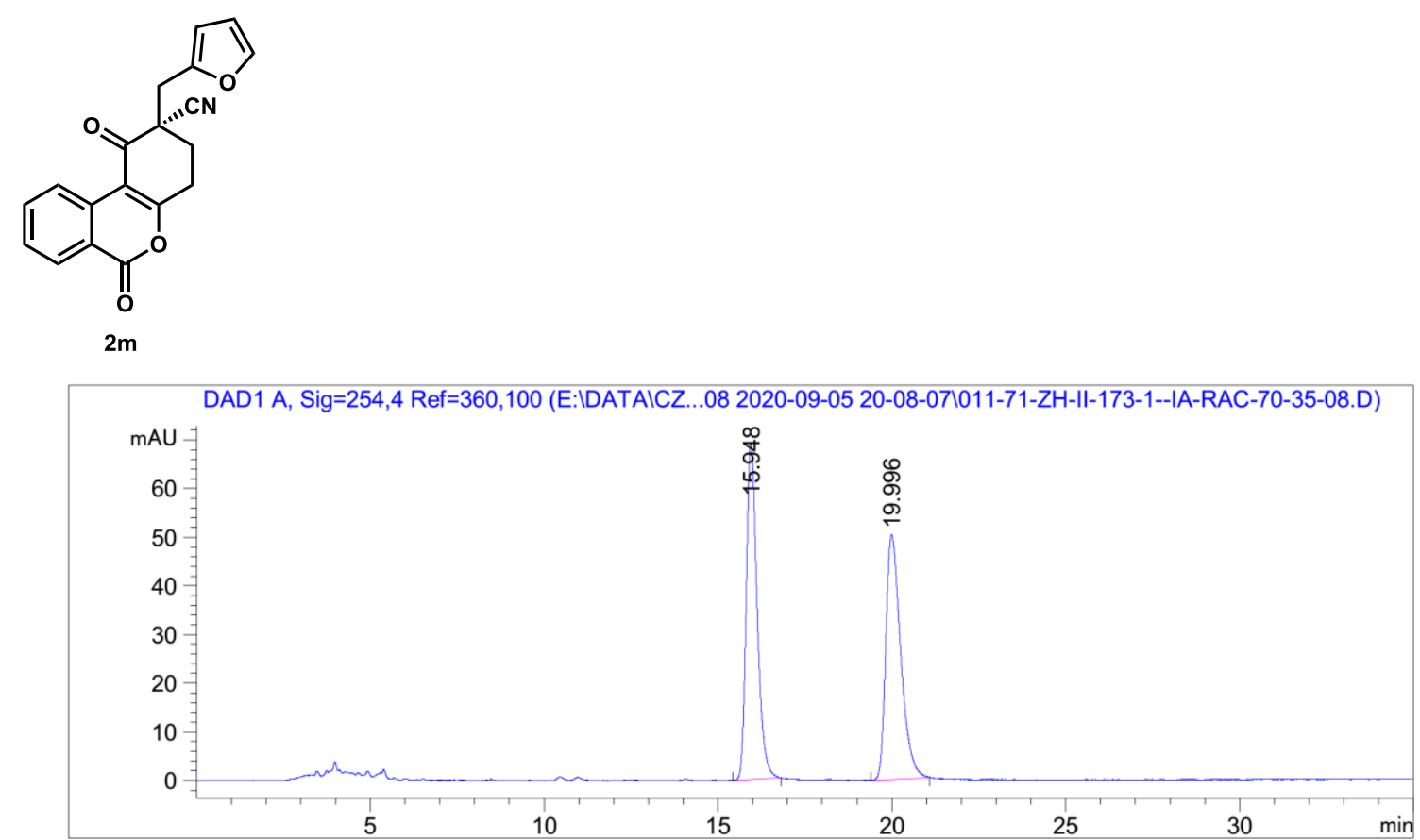

Signal 1: DAD1 A, Sig=254,4 Ref $=360,100$
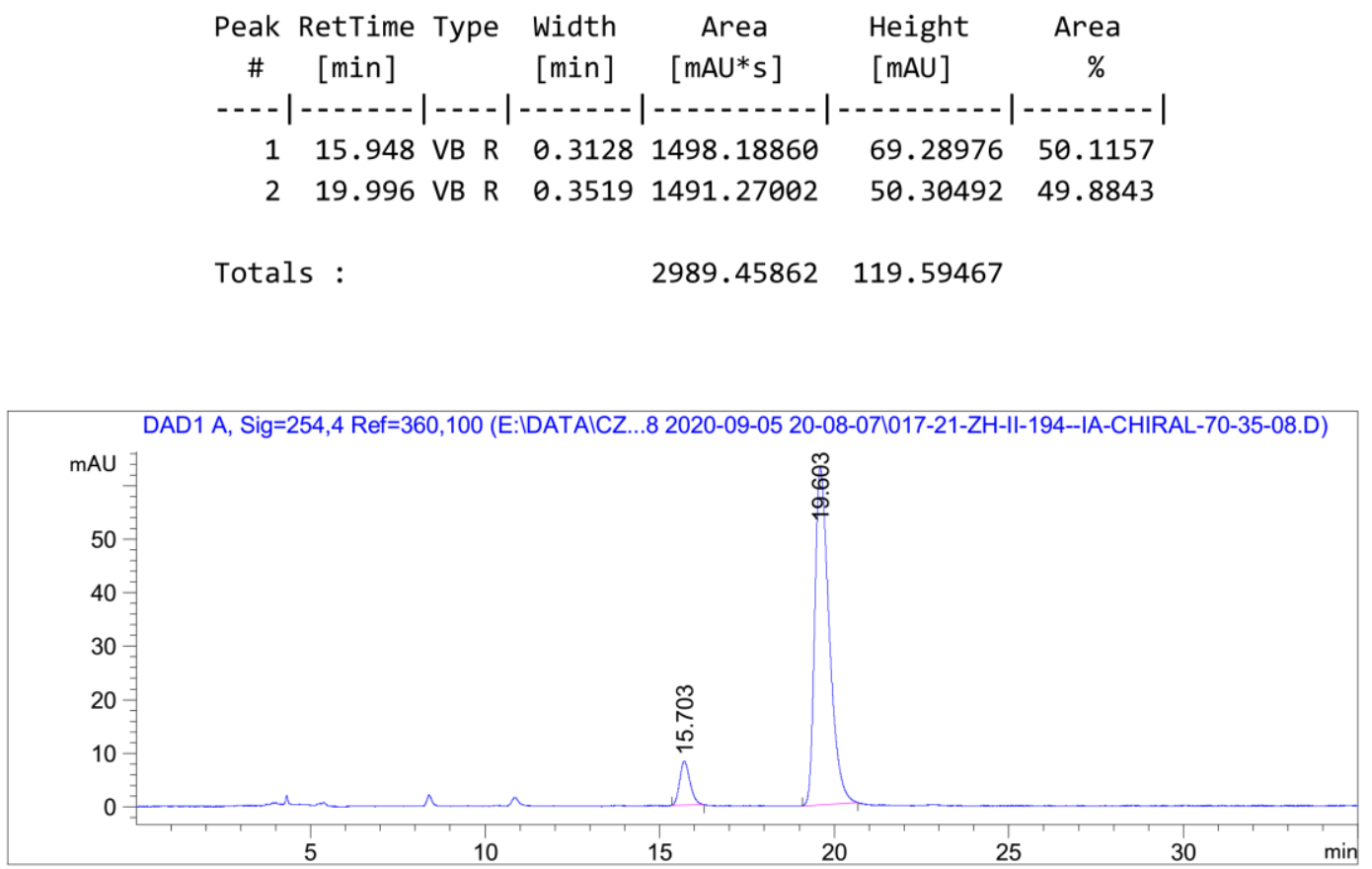

Signal 1: DAD1 A, Sig=254,4 Ref=360,100

\begin{tabular}{|c|c|c|c|c|c|c|}
\hline $\begin{array}{c}\text { Peak } \\
\#\end{array}$ & $\begin{array}{c}\text { RetTime } \\
\text { [min] }\end{array}$ & Type & $\begin{array}{l}\text { Width } \\
\text { [min] }\end{array}$ & $\begin{array}{c}\text { Area } \\
{\left[\mathrm{mAU}^{*} \mathrm{~s}\right]}\end{array}$ & $\begin{array}{l}\text { Height } \\
{[\mathrm{mAU}]}\end{array}$ & $\begin{array}{c}\text { Area } \\
\%\end{array}$ \\
\hline- & 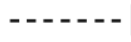 & 1 & & - & | & 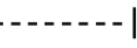 \\
\hline 1 & 15.703 & BV $R$ & 0.2449 & 170.29576 & 8.21413 & 8.5753 \\
\hline 2 & 19.603 & BB & 0.3520 & 1815.59424 & 62.94141 & 91.4247 \\
\hline 0 & : & & & 1985.89000 & 71.15554 & \\
\hline
\end{tabular}


<smiles>CC(=O)CC[C@]1(C#N)CCc2oc(=O)c3ccccc3c2C1=O</smiles>

2n

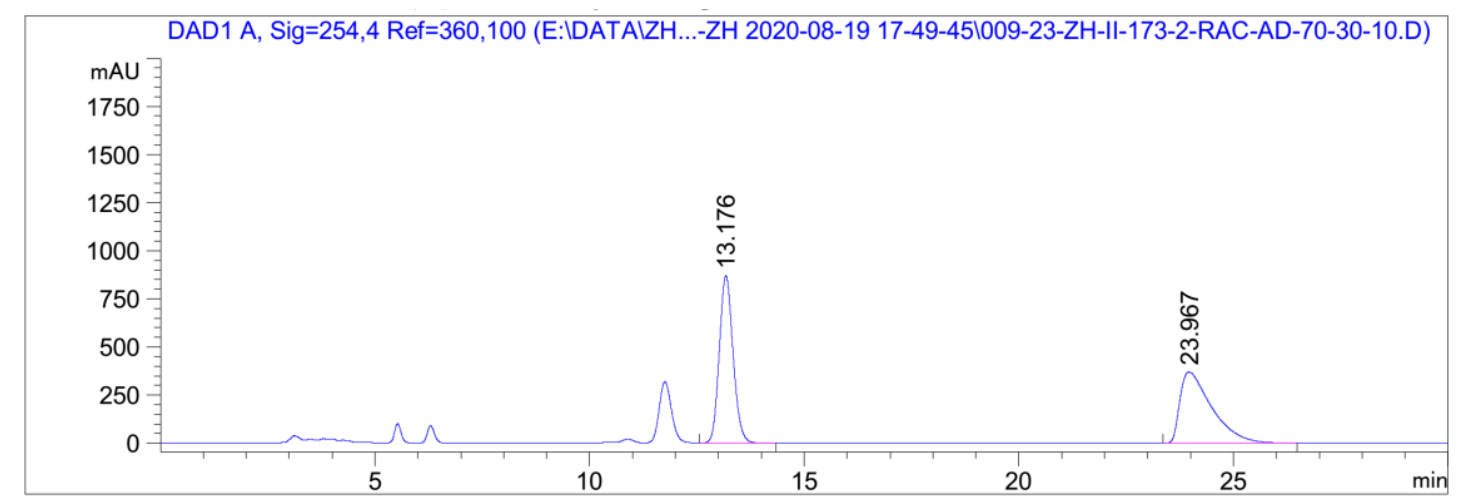

Signal 1: DAD1 A, Sig=254,4 Ref=360,100

\begin{tabular}{|c|c|c|c|c|c|c|}
\hline $\begin{array}{c}\text { Peak } \\
\quad \#\end{array}$ & $\begin{array}{c}\text { RetTime } \\
\text { [min] }\end{array}$ & Type & $\begin{array}{l}\text { Width } \\
\text { [min] }\end{array}$ & $\begin{array}{c}\text { Area } \\
{\left[\mathrm{mAU}^{*} \mathrm{~s}\right]}\end{array}$ & $\begin{array}{l}\text { Height } \\
{[\mathrm{mAU}]}\end{array}$ & $\begin{array}{c}\text { Area } \\
\%\end{array}$ \\
\hline & & & & $1-$ & -- & --- \\
\hline 1 & 13.176 & BB & 0.3439 & $1.94148 \mathrm{e} 4$ & 869.99915 & 50.5265 \\
\hline 2 & 23.967 & BB & 0.7099 & $1.90102 \mathrm{e} 4$ & 369.68668 & 49.4735 \\
\hline To & . & & & $3.84250 \mathrm{e} 4$ & 68582 & \\
\hline
\end{tabular}

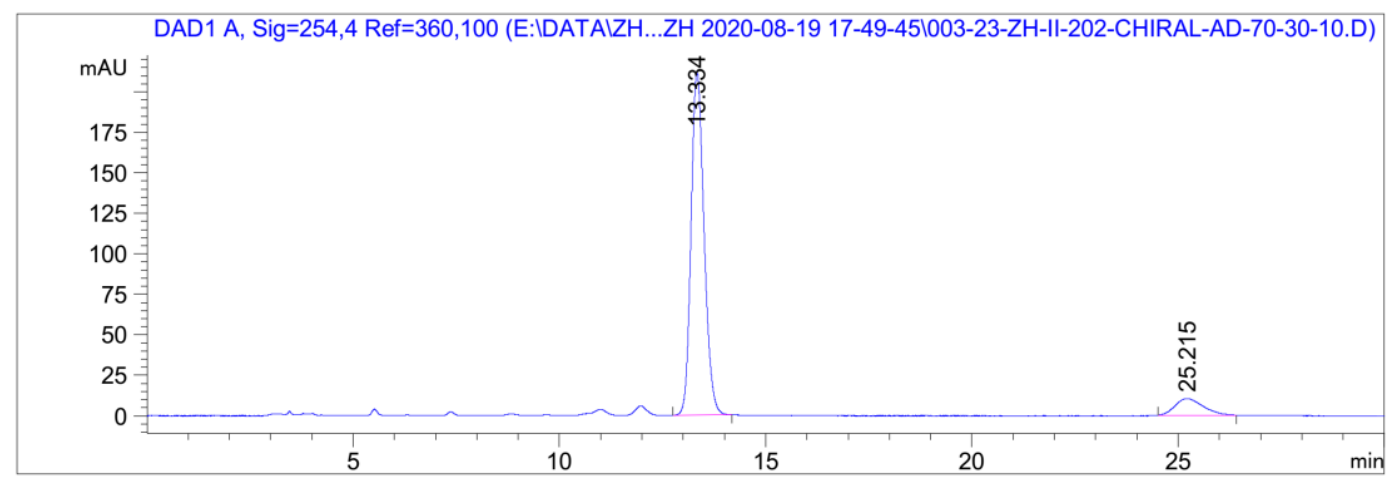

Signal 1: DAD1 A, Sig=254,4 Ref=360,100

\begin{tabular}{|c|c|c|c|c|c|c|}
\hline $\begin{array}{c}\text { Peak } \\
\quad \#\end{array}$ & $\begin{array}{c}\text { RetTime } \\
\text { [min] }\end{array}$ & Type & $\begin{array}{l}\text { Width } \\
\text { [min] }\end{array}$ & $\begin{array}{c}\text { Area } \\
{\left[\mathrm{mAU}^{*} \mathrm{~s}\right]}\end{array}$ & $\begin{array}{l}\text { Height } \\
{[\mathrm{mAU}]}\end{array}$ & $\begin{array}{c}\text { Area } \\
\%\end{array}$ \\
\hline & & & & -- & $=-$ & ---8 \\
\hline 1 & 13.334 & BB & 0.3363 & 4647.85010 & 211.97200 & 90.4854 \\
\hline 2 & 25.215 & BB & 0.5571 & 488.72659 & 10.27228 & 9.5146 \\
\hline To & 15 . & & & 5136.57669 & 222.2 & \\
\hline
\end{tabular}


<smiles>C[C@@]1(CCC#N)CCc2oc(=O)c3ccccc3c2C1=O</smiles>

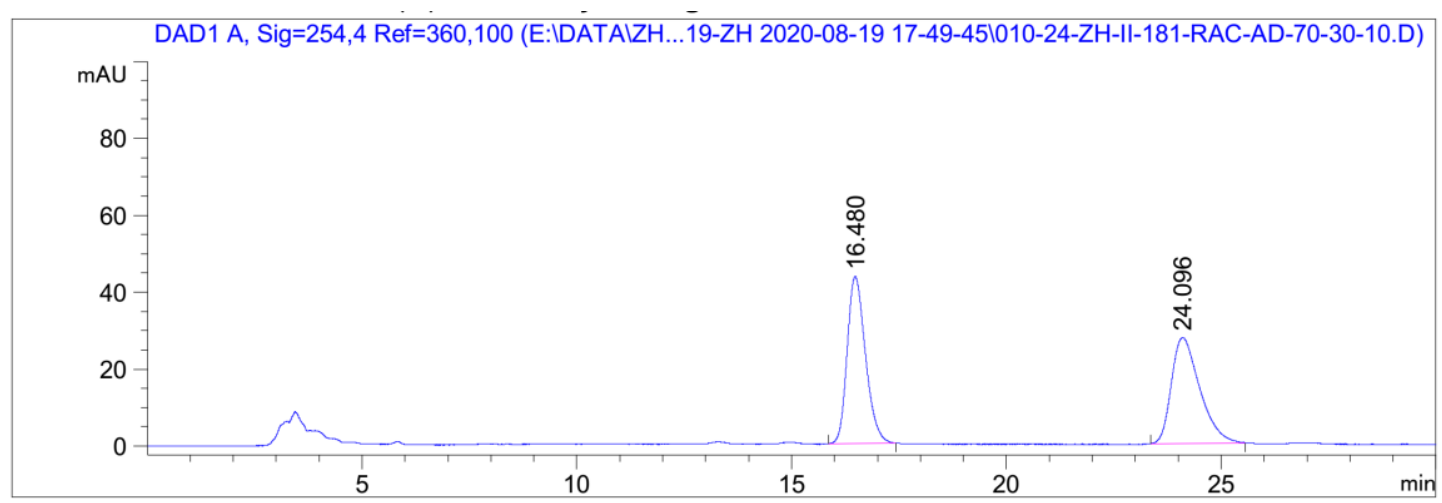

Signal 1: DAD1 A, Sig=254,4 Ref $=360,100$

\begin{tabular}{|c|c|c|c|c|c|c|}
\hline $\begin{array}{c}\text { Peak } \\
\#\end{array}$ & $\begin{array}{c}\text { RetTime } \\
\text { [min] }\end{array}$ & Type & $\begin{array}{l}\text { Width } \\
\text { [min] }\end{array}$ & $\begin{array}{c}\text { Area } \\
{\left[\mathrm{mAU}^{*} \mathrm{~s}\right]}\end{array}$ & $\begin{array}{l}\text { Height } \\
{[\mathrm{mAU}]}\end{array}$ & $\begin{array}{c}\text { Area } \\
\%\end{array}$ \\
\hline 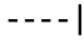 & & & & -- & -- & - \\
\hline 1 & 16.4 & BB & 27 & 1285.18274 & 43.43372 & 50.4620 \\
\hline 2 & 24.096 & BB & .5379 & 1261.65088 & 27.51744 & 49.5380 \\
\hline
\end{tabular}

Totals : $\quad 2546.83362 \quad 70.95115$

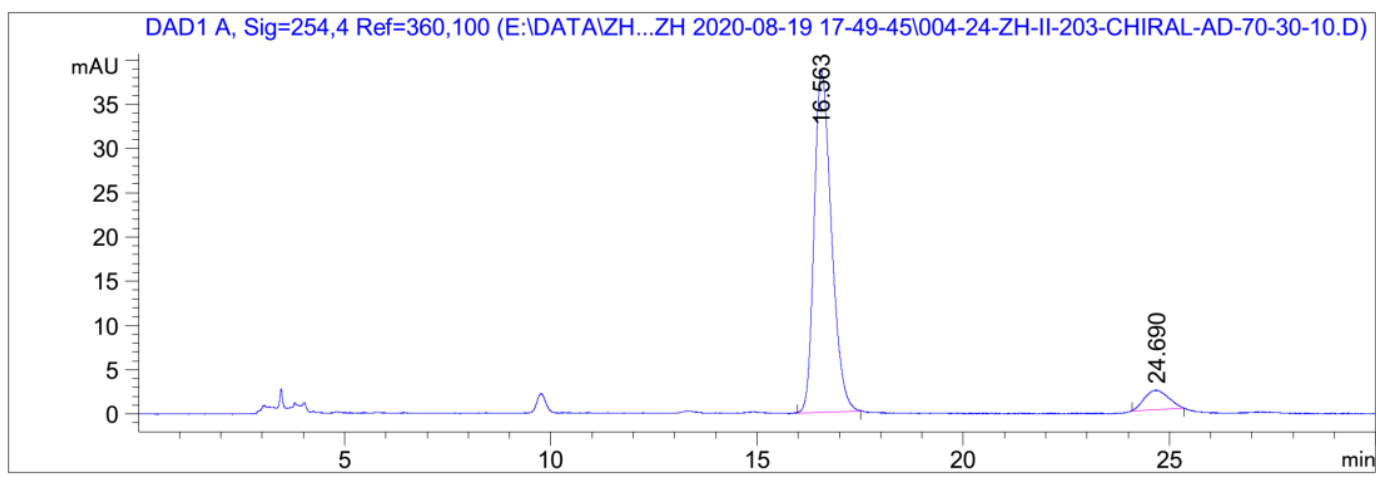

Signal 1: DAD1 A, Sig=254,4 Ref=360,100

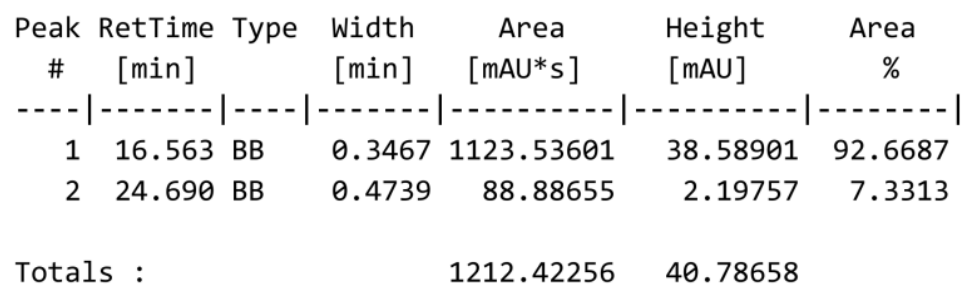



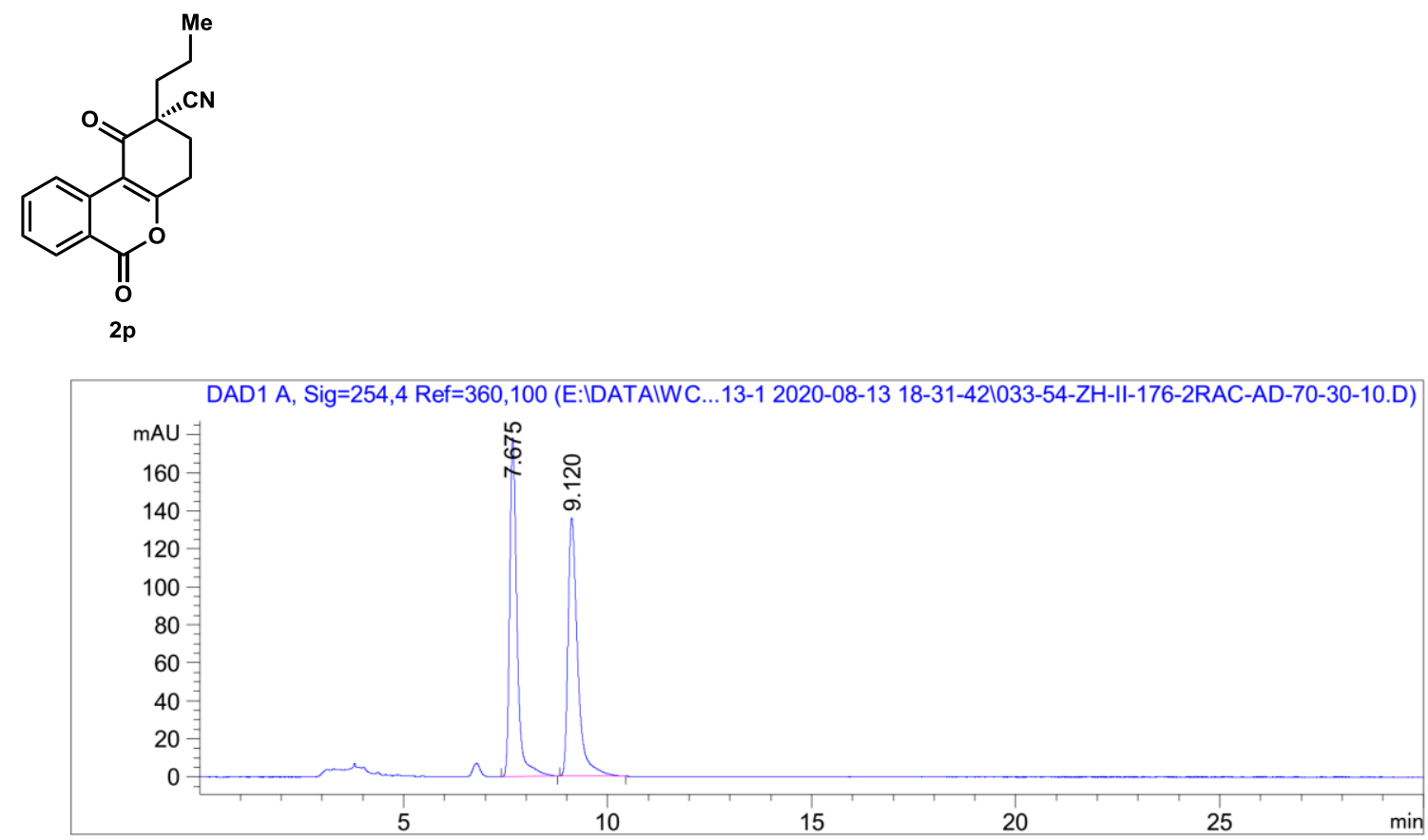

Signal 1: DAD1 A, Sig=254,4 Ref $=360,100$

\begin{tabular}{|c|c|c|c|c|c|c|}
\hline $\begin{array}{c}\text { Peak } \\
\#\end{array}$ & $\begin{array}{c}\text { RetTime } \\
\text { [min] }\end{array}$ & Type & $\begin{array}{l}\text { Width } \\
\text { [min] }\end{array}$ & $\begin{array}{c}\text { Area } \\
{\left[\mathrm{mAU}^{*} \mathrm{~s}\right]}\end{array}$ & $\begin{array}{l}\text { Height } \\
\text { [mAU] }\end{array}$ & $\begin{array}{c}\text { Area } \\
\%\end{array}$ \\
\hline---1 &.--- & & -- & |------- & - & | \\
\hline 1 & 7.675 & BB & 0.1881 & 2232.34766 & 178.27304 & 50.0362 \\
\hline 2 & 9.120 & BB & 0.2387 & 2229.11743 & 135.98341 & 49.9638 \\
\hline Tota] & $:$ & & & 4461.46509 & 314.25645 & \\
\hline
\end{tabular}

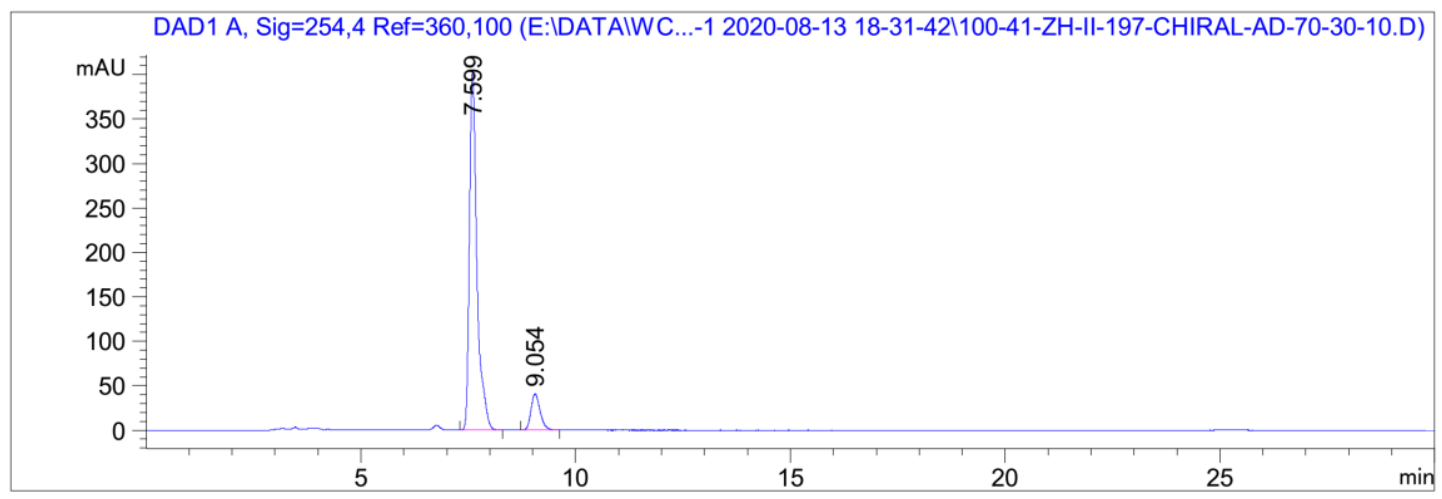

Signal 1: DAD1 A, Sig=254,4 Ref=360,100

\begin{tabular}{|c|c|c|c|c|c|c|}
\hline $\begin{array}{c}\text { Peak } \\
\#\end{array}$ & $\begin{array}{c}\text { RetTime } \\
\text { [min] }\end{array}$ & Type & $\begin{array}{l}\text { Width } \\
\text { [min] }\end{array}$ & $\begin{array}{c}\text { Area } \\
{\left[\mathrm{mAU}^{*} \mathrm{~s}\right]}\end{array}$ & $\begin{array}{l}\text { Height } \\
{[\mathrm{mAU}]}\end{array}$ & $\begin{array}{c}\text { Area } \\
\%\end{array}$ \\
\hline & & & & & & \\
\hline 1 & 7.5 & B & 352 & 4939.94482 & 402.38495 & 89.1443 \\
\hline 2 & 9.054 & BB & 2191 & 601.56891 & 40.77797 & 10.8557 \\
\hline
\end{tabular}

Totals :

$5541.51373 \quad 443.16292$ 
<smiles>C=CCC1(C)CCc2oc(=O)c3ccccc3c2C1=O</smiles>

$2 q$

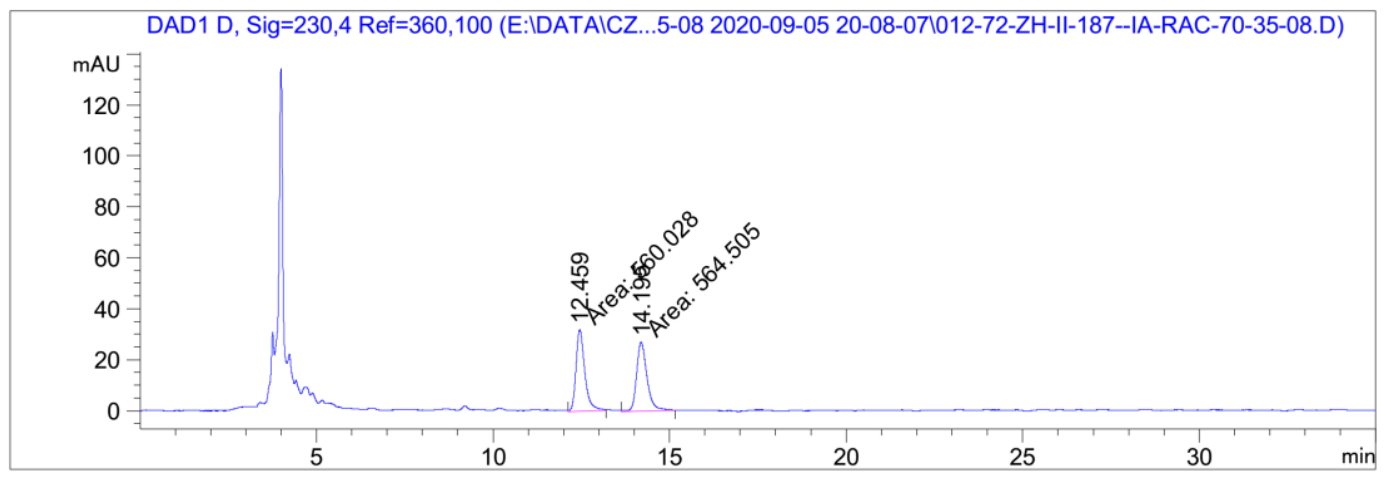

Signal 1: DAD1 D, Sig=230,4 Ref $=360,100$

\begin{tabular}{|c|c|c|c|c|c|c|}
\hline $\begin{array}{c}\text { Peak } \\
\#\end{array}$ & $\begin{array}{l}\text { RetTime } \\
\text { [min] }\end{array}$ & Type & $\begin{array}{l}\text { Width } \\
\text { [min] }\end{array}$ & $\begin{array}{c}\text { Area } \\
{\left[\mathrm{mAU}^{*} \mathrm{~s}\right]}\end{array}$ & $\begin{array}{l}\text { Height } \\
{[\mathrm{mAU}]}\end{array}$ & $\begin{array}{c}\text { Area } \\
\%\end{array}$ \\
\hline -- & & & & --- & & - \\
\hline 1 & 12.459 & MM & 0.2907 & 560.02771 & 32.10292 & 49.8009 \\
\hline 2 & 14.195 & MM & 0.3463 & 564.50494 & 27.16977 & 50.1991 \\
\hline otc & : & & & 1124.53265 & 59.2 & \\
\hline
\end{tabular}

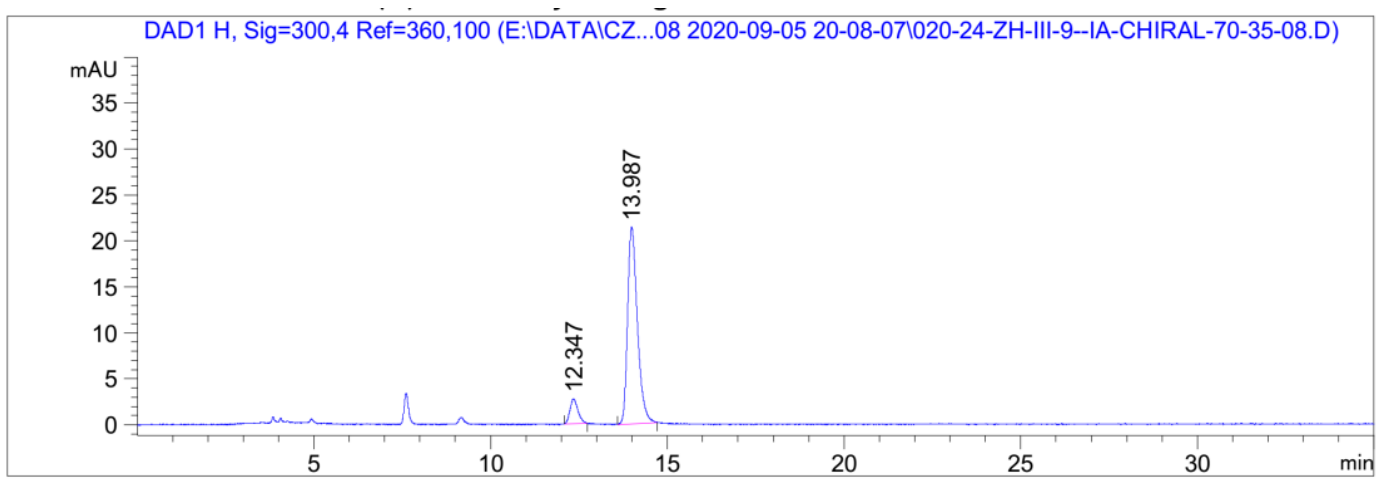

Signal 1: DAD1 H, Sig=300,4 Ref=360,100

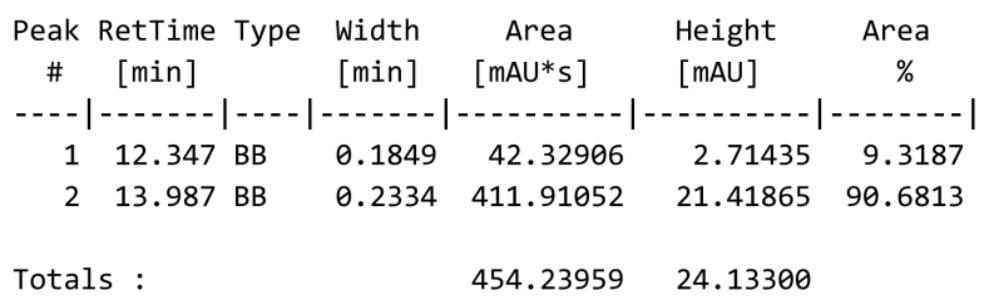



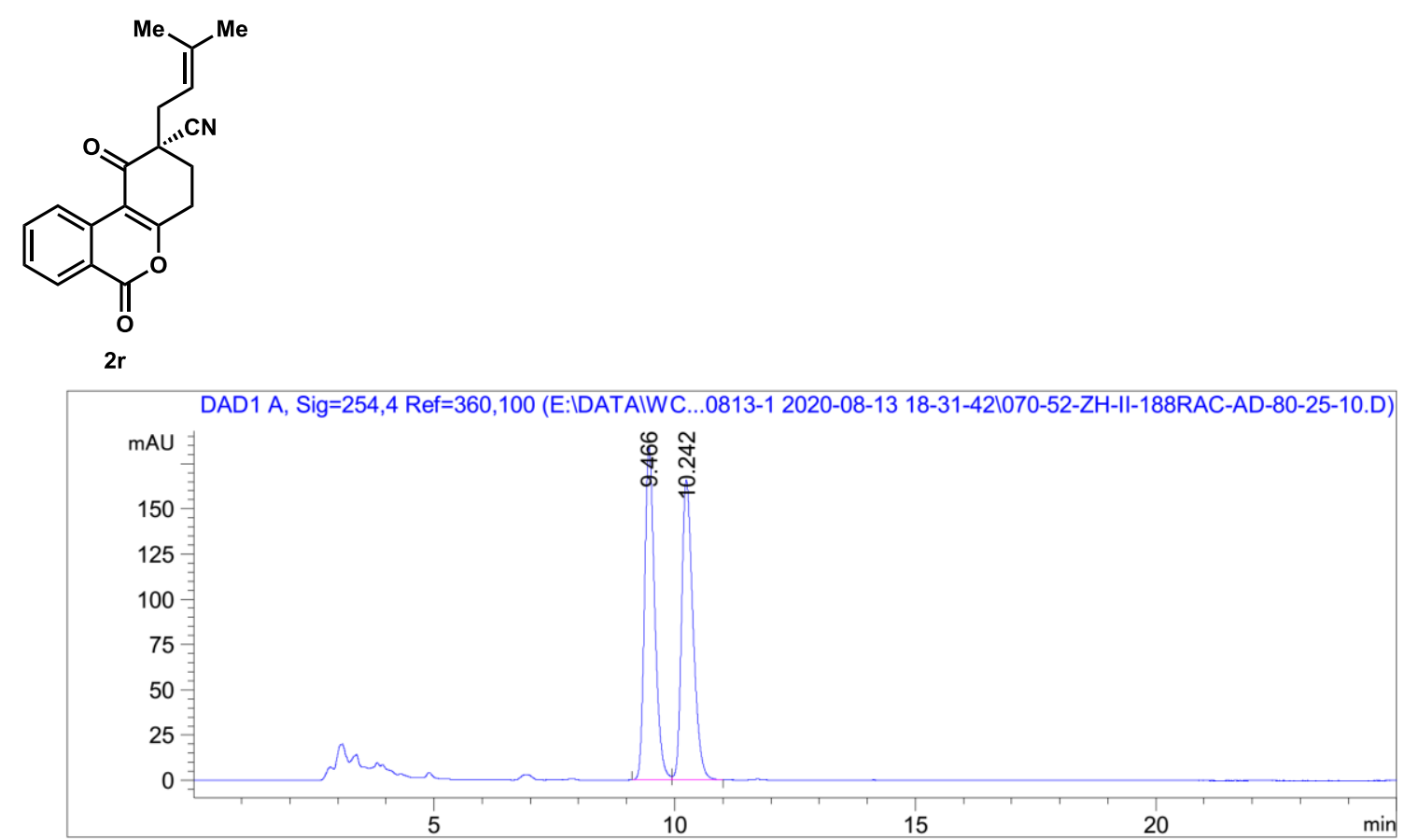

Signal 1: DAD1 A, Sig=254, 4 Ref $=360,100$

\begin{tabular}{|c|c|c|c|c|c|c|}
\hline $\begin{array}{c}\text { Peak } \\
\#\end{array}$ & $\begin{array}{c}\text { RetTime } \\
\text { [min] }\end{array}$ & Type & $\begin{array}{l}\text { Width } \\
\text { [min] }\end{array}$ & $\begin{array}{c}\text { Area } \\
{[\mathrm{mAU} * \mathrm{~s}]}\end{array}$ & $\begin{array}{l}\text { Height } \\
\text { [mAU] }\end{array}$ & $\begin{array}{c}\text { Area } \\
\%\end{array}$ \\
\hline - & -- & & -- & $\mid--1$ & 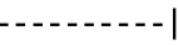 & | \\
\hline 1 & 9.466 & BV & 0.2238 & 2706.51929 & 183.76343 & 49.8953 \\
\hline 2 & 10.242 & VB & 0.2504 & 2717.88184 & 165.66042 & 50.1047 \\
\hline ced & : & & & 5424.40112 & 349.42384 & \\
\hline
\end{tabular}

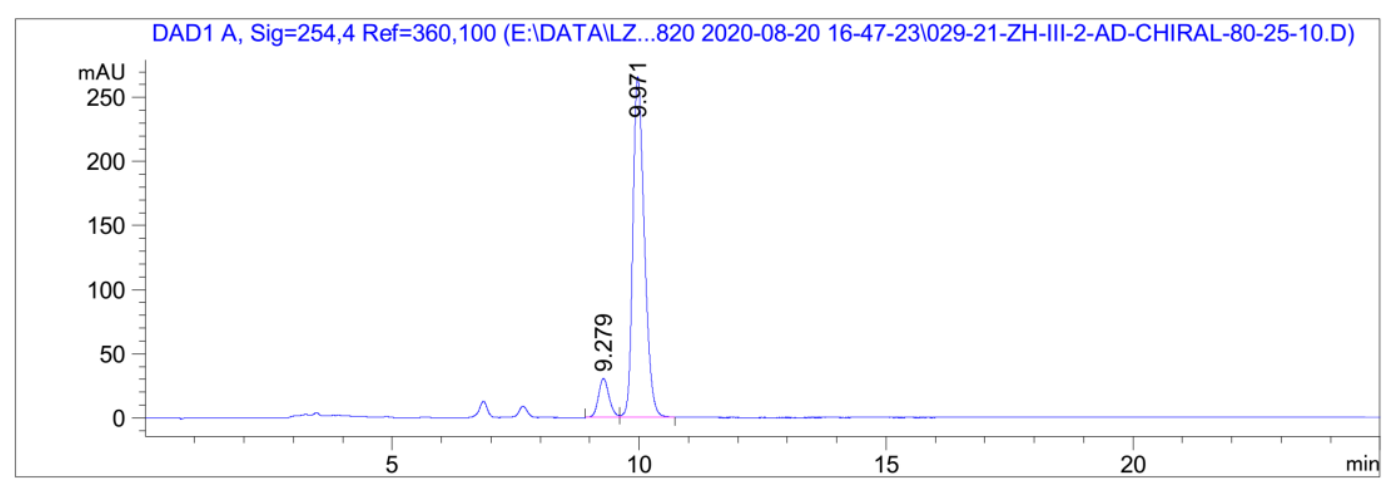

Signal 1: DAD1 A, Sig $=254,4$ Ref $=360,100$

\begin{tabular}{|c|c|c|c|c|c|c|}
\hline $\begin{array}{c}\text { Peak } \\
\#\end{array}$ & $\begin{array}{c}\text { RetTime } \\
\text { [min] }\end{array}$ & Type & $\begin{array}{l}\text { Width } \\
\text { [min] }\end{array}$ & $\begin{array}{c}\text { Area } \\
{\left[\mathrm{mAU}^{*} \mathrm{~s}\right]}\end{array}$ & $\begin{array}{l}\text { Height } \\
{[\mathrm{mAU}]}\end{array}$ & $\begin{array}{c}\text { Area } \\
\%\end{array}$ \\
\hline 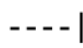 & 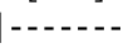 & & - & |-.... & 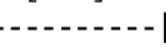 & - \\
\hline 1 & 9.279 & BV & 0.2110 & 457.75220 & 30.51668 & 9.6155 \\
\hline 2 & 9.971 & VB & 0.2453 & 4302.79346 & 265.92203 & 90.3845 \\
\hline Total & Ls: & & & 4760.54565 & 296.43870 & \\
\hline
\end{tabular}


<smiles>N#C[C@@]1(Br)CCCc2oc(=O)c3ccccc3c2C1=O</smiles>

2s

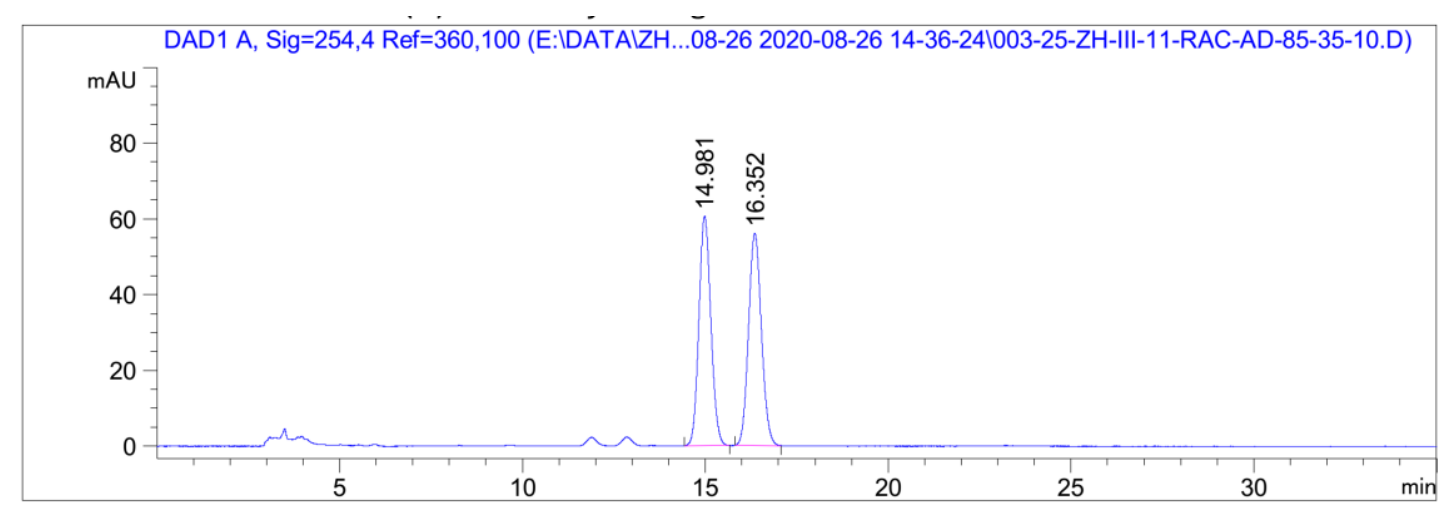

Signal 1: DAD1 A, Sig=254,4 Ref=360,100

Peak RetTime Type Width Area Height Area

\# [min] [min] [mAU*s $] \quad[\mathrm{mAU}] \%$

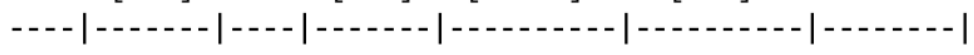

$\begin{array}{lllllll}1 & 14.981 & \text { BB } & 0.2886 & 1377.38745 & 60.61434 & 50.1020 \\ 2 & 16.352 & \text { BB } & 0.3204 & 1371.78149 & 56.08198 & 49.8980\end{array}$

Totals :

$2749.16895 \quad 116.69632$

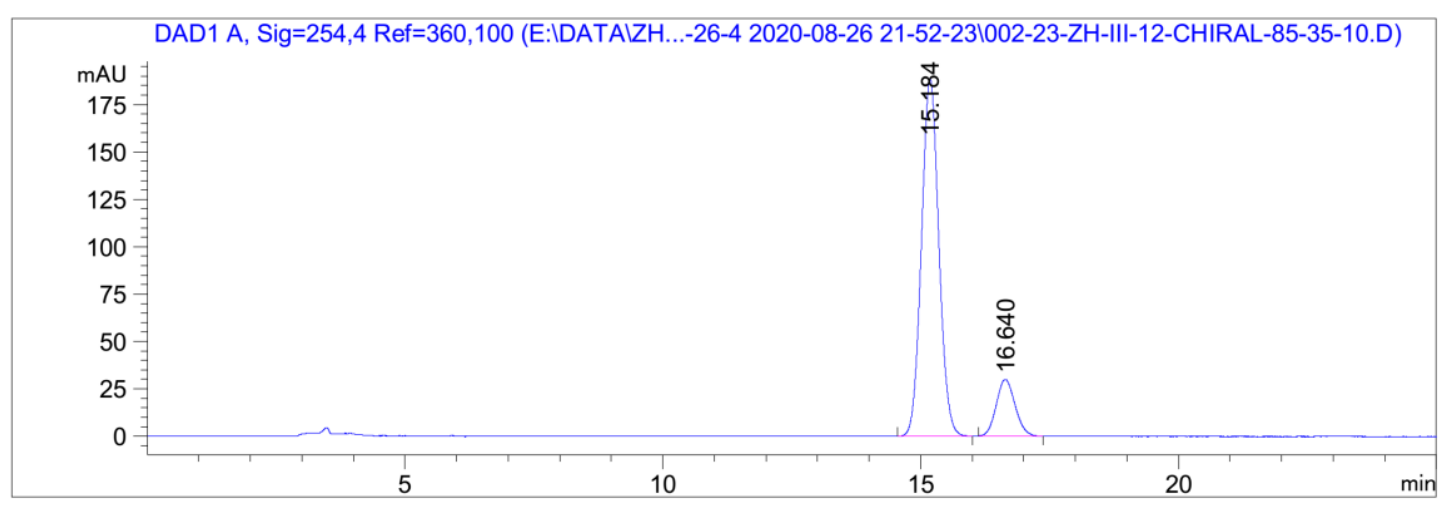

Signal 1: DAD1 A, Sig=254,4 Ref=360,100

\begin{tabular}{|c|c|c|c|c|c|c|}
\hline $\begin{array}{c}\text { Peak } \\
\#\end{array}$ & $\begin{array}{c}\text { RetTime } \\
\text { [min] }\end{array}$ & Type & $\begin{array}{l}\text { Width } \\
\text { [min] }\end{array}$ & $\begin{array}{c}\text { Area } \\
{\left[\mathrm{mAU}^{*} \mathrm{~s}\right]}\end{array}$ & $\begin{array}{l}\text { Height } \\
{[\mathrm{mAU}]}\end{array}$ & $\begin{array}{c}\text { Area } \\
\%\end{array}$ \\
\hline & & & & |-- & -- & --- \\
\hline 1 & 15.184 & BB & 0.3485 & 4412.54199 & 188.56168 & 85.3942 \\
\hline 2 & 16.640 & BB & 0.3020 & 754.71924 & 29.84479 & 14.6058 \\
\hline Total & : & & & 5167.26123 & 218.40646 & \\
\hline
\end{tabular}




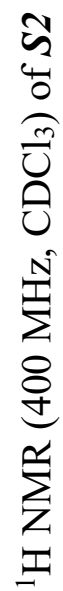

$190^{\circ} \varepsilon$

$6 \angle 0^{\circ} \varepsilon$

$860^{\circ} \varepsilon$

เE์'

$6 \nabla \varepsilon^{\circ} \varepsilon$

$89 \varepsilon^{\circ} \varepsilon$

$976^{\circ} \varepsilon-$

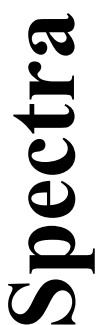

09Z L

乙EE $\angle$

GE ' $L$

ISE $L$

$\nabla G \mathcal{E}^{\circ} L$

$0 \angle \varepsilon^{\circ} \angle$

$\varepsilon \angle \varepsilon^{\circ} L$

9Zち $L$

$0 \varepsilon \nabla^{\circ} L$

Stt' $L$

$6 \nabla \nabla^{\circ} L$

† $9 \nabla^{\circ} L$

$\angle 9 \nabla^{\circ} \angle$

$\angle \forall G^{\circ} \angle$

IGG 2

$\angle 99^{\circ} \mathrm{L}$

$0 \angle G^{\circ} \angle$

$868^{\circ} \mathrm{L}$

$206^{\circ} \mathrm{L}$

$816 . \mathrm{L}$

ZZ6 L

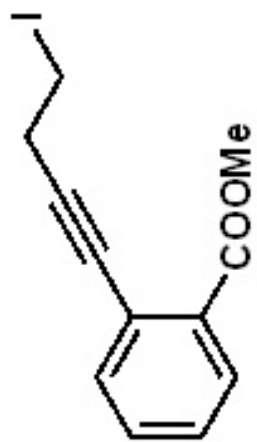




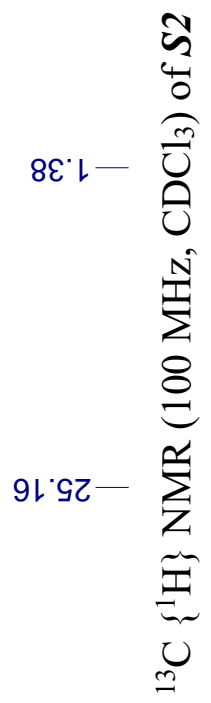

9ع'ح९-

$78.9 L$

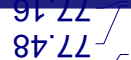

OL.

88' $86-$

98'ย乙レ

$88^{\circ} \angle Z L$

乙† $0 \varepsilon \downarrow$

$08 \cdot L L$

00'乙E

$0 S^{\circ} \nabla \varepsilon L$

†8.99l-
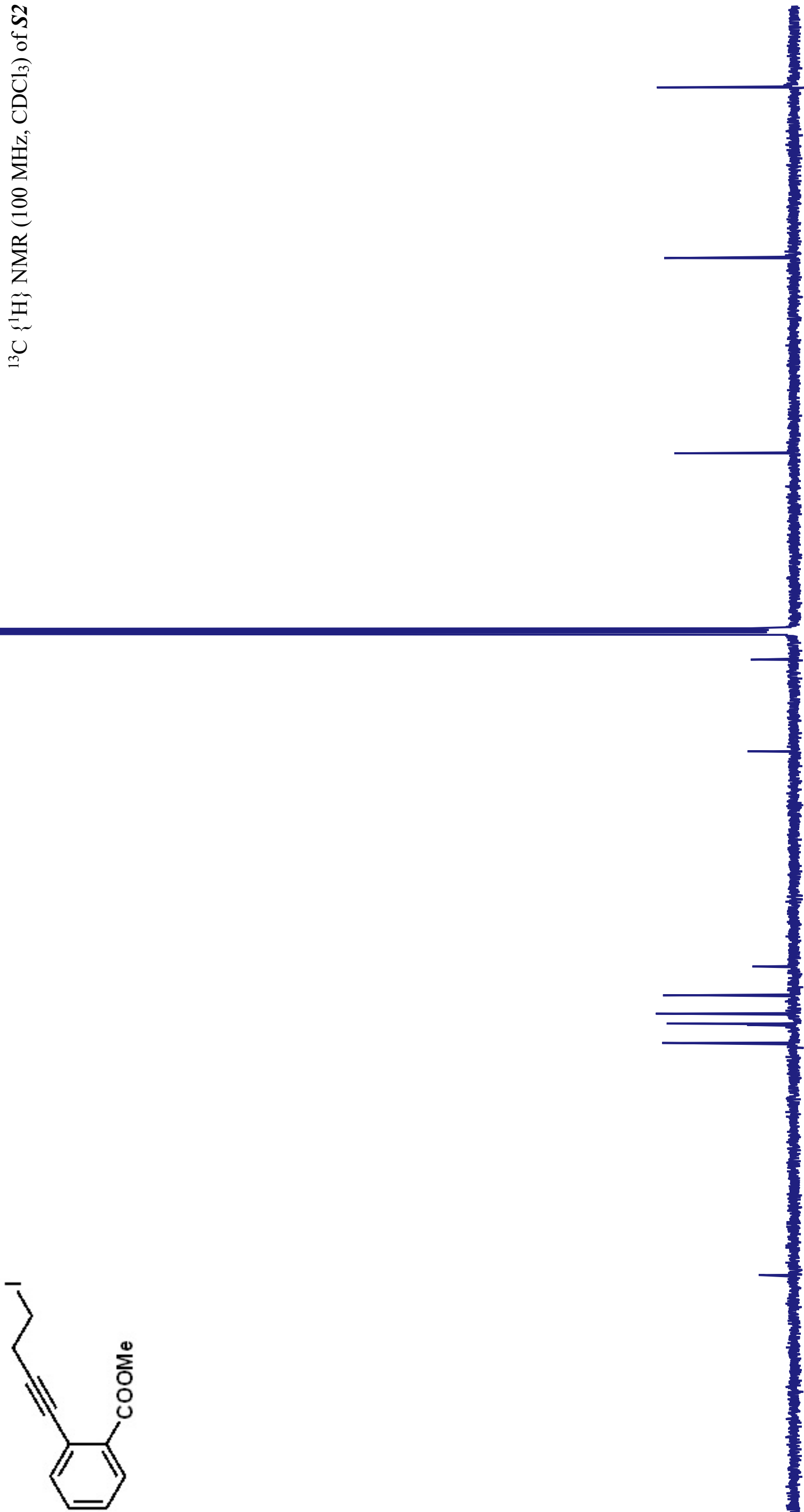
9عย'乙

$092 \angle$ 99E L $09 \varepsilon^{\circ} \angle$ $\neg L E^{\circ} L$ $8 L \varepsilon^{\circ} L$ $\varepsilon 6 \varepsilon^{\circ} L$ $\angle 6 \varepsilon^{\circ} \angle$ $\nabla S \nabla^{\circ} L$ $\angle G D^{\circ} L$ $\varepsilon \angle \nabla^{\circ} L$ $\angle L D^{\circ} L$ $16 t^{\circ} L$ $\checkmark 67^{\circ} L$ $009^{\circ} \mathrm{L}$ $\checkmark 09^{\circ} \mathrm{L}$ $6 \mathrm{IS}^{\circ} \mathrm{L}$ $\nabla Z 9^{\circ} \mathrm{L}$ $6766^{\circ} \mathrm{L}$ ES6 $L$ $696^{\circ} \mathrm{L}$ ZL6 L
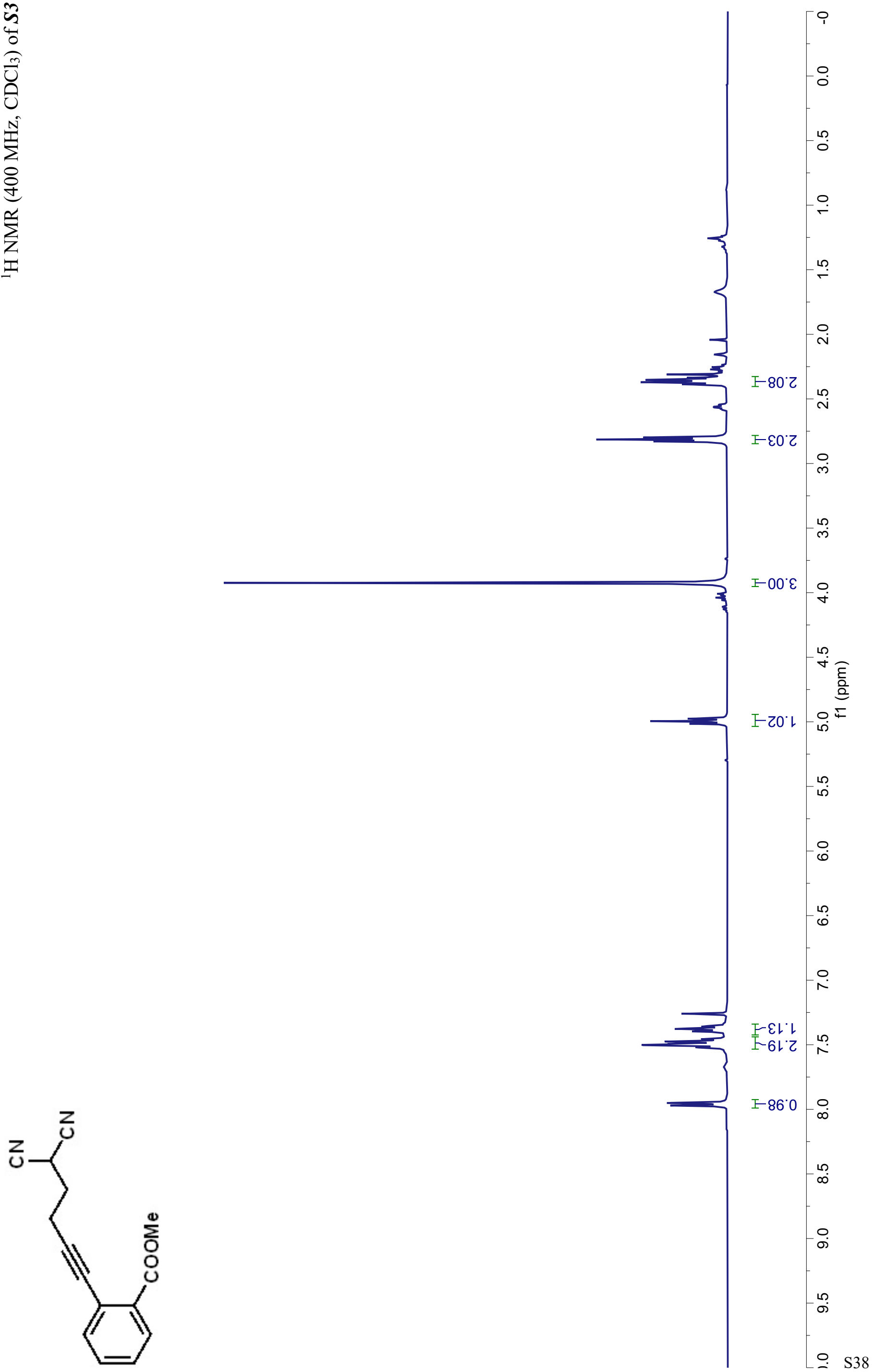
st.LL-

OS'ZS-

$78.9 L$

$9 L^{\circ} L L$

งเ'E8-

เย.06-

ยเยレー

99'E乙

乙Z $8 Z$ L

89.0हL

GL'LL

†レ乙EL

$\varepsilon 0^{\circ} \nabla \varepsilon\llcorner-$
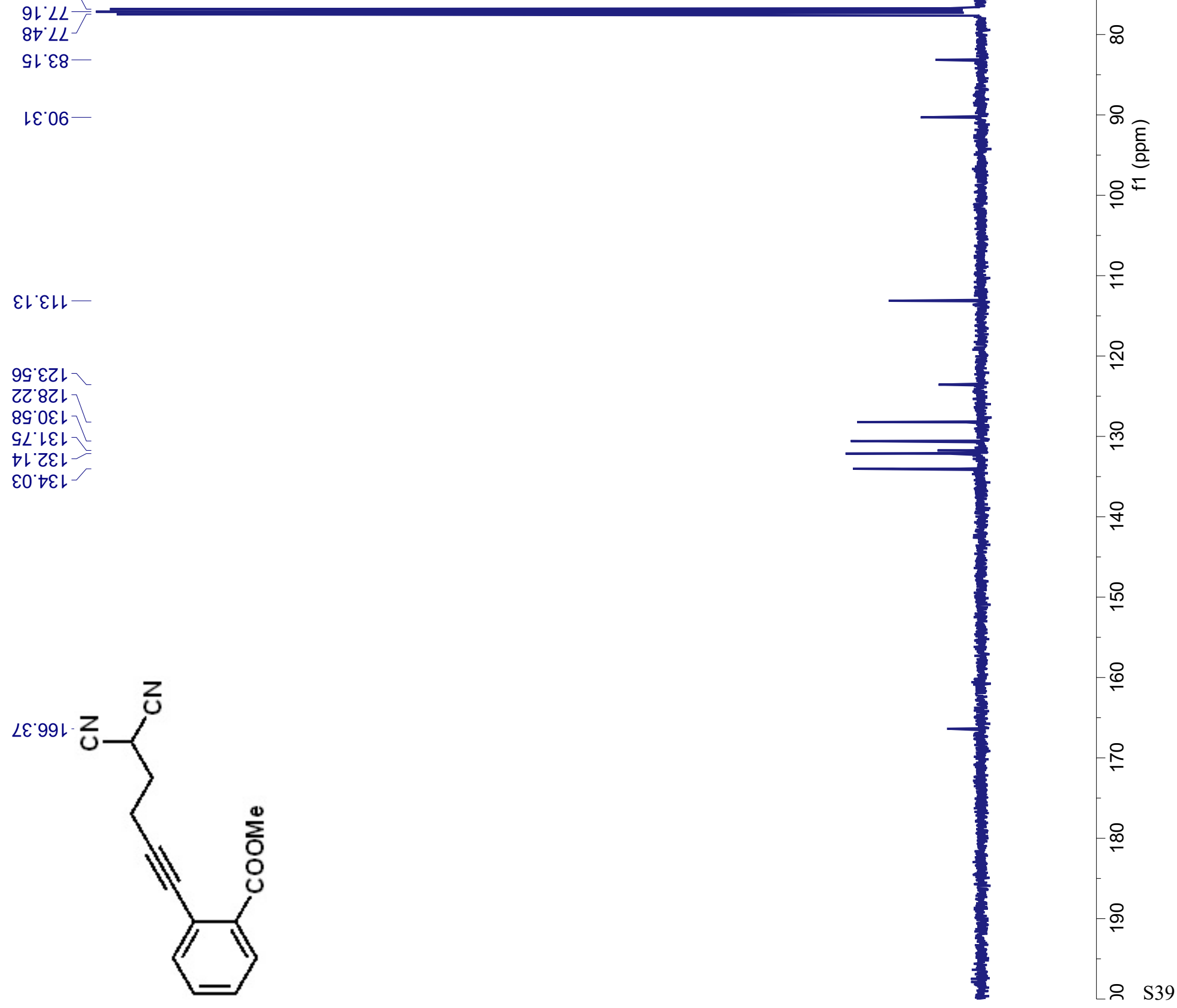


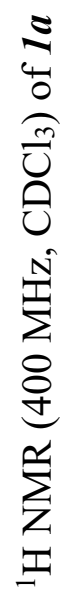

งย次

ธ๑ย'乙

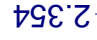

$89 \varepsilon^{\prime} \mathrm{Z}$

$99 \varepsilon$ '

$\nabla \angle \varepsilon^{\prime} Z$

$906^{\circ} \mathrm{Z}$

126'Z

926 Z

ฤ $6^{\circ} 乙$

$90 \varepsilon^{\circ} \varepsilon$

$106^{\circ} \varepsilon$

$09 Z^{\circ} L$

$\nabla \varepsilon \varepsilon^{\circ} L$

8E ' $L$

ESE $\angle$

$\angle G E \cdot L$

乙LE' $\angle$

$9 \angle E^{\circ} L$

OLt $L$

8Lt L

LEt $L$

SEt $L$

OSt $L$

$\nabla G t^{\circ} L$

$69 t^{\circ} \mathrm{L}$

$\downarrow \varepsilon G^{\circ} L$

8EG $L]$

$\checkmark G G^{\circ} L$

$\angle G G^{\circ} L$

$\angle 06^{\circ} \mathrm{L}$

OL6'
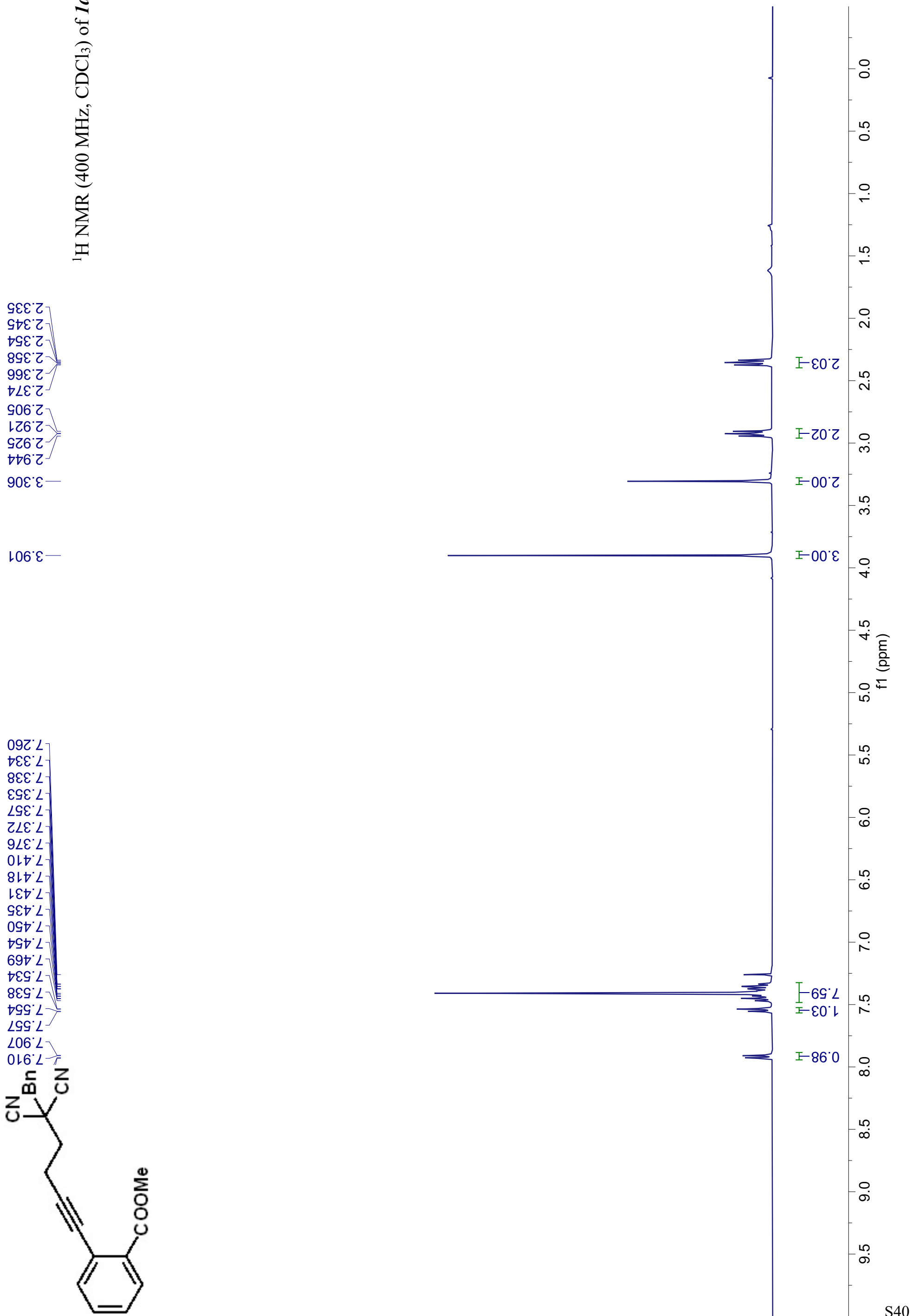
$96.91-\underbrace{8}$

$8279-$

$78 \cdot 9 L$

$9 L^{\circ} L L$

$87^{\circ} \angle L$

$\mathrm{S}^{\circ} 18^{-}$

$98.06-$

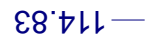

99'E乙L

L $0.82 \mathrm{~L}$

$70.6 \mathrm{LL}$

ㄴ.62L

レナ0Eレ

$\neg 8^{\circ}\llcorner\varepsilon L$

$\angle 8^{\circ} L E L$

乙o'乙ह

$9 \varepsilon^{\circ} \nabla \varepsilon\llcorner$
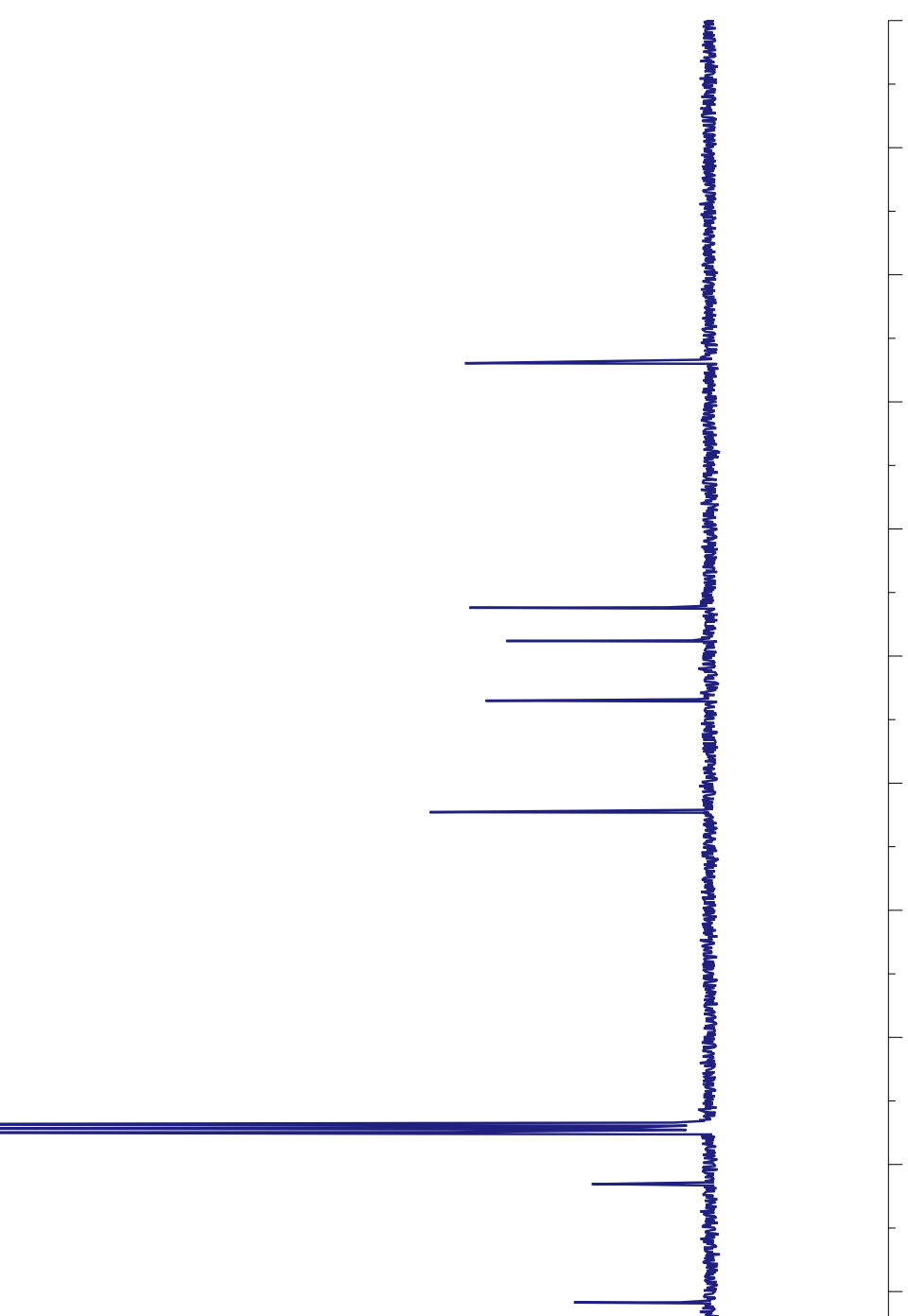

$\infty$

요

ํㅡㄹ

음

읃

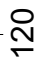

$\stackrel{n}{\circ}$

운

욤

$\stackrel{\circ}{\circ}$

$09.991-2 \frac{1}{0}$ 


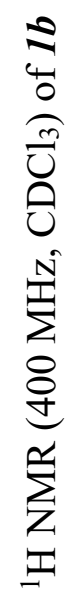

8乙ย'

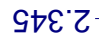

$\angle \nabla \varepsilon ' Z$

LSE'

$\angle 9 \varepsilon$ '

$G \angle E^{\prime} Z$

$268^{\circ} \mathrm{Z}$

$006^{\circ} \mathrm{Z}$

$806^{\circ} \mathrm{Z}$

2เ6 2

จเ6 2

乙Z6 乙

เE6 2

$0 \mathcal{E}^{\circ} \varepsilon^{-}$

乙68 ${ }^{\circ}$

$\angle D Z L$

$09 Z 2$

ISZ $L$

$09 Z^{\circ} \mathrm{L}$

$+9 Z^{\circ} \mathrm{L}$

$99 Z^{\circ} \mathrm{L}$

$69 Z^{\circ} L$

ILZ' $L$

$\angle 6 \varepsilon^{\circ} \angle$

$\varepsilon 0 t^{\circ} \mathrm{L}$

$80 t^{\circ} L$

8เV'L

$8 \varepsilon \nabla^{\circ} L$

$\varepsilon Z L L$

GZL L

9ZL L L

$0 \varepsilon L L$

IEL L $L$
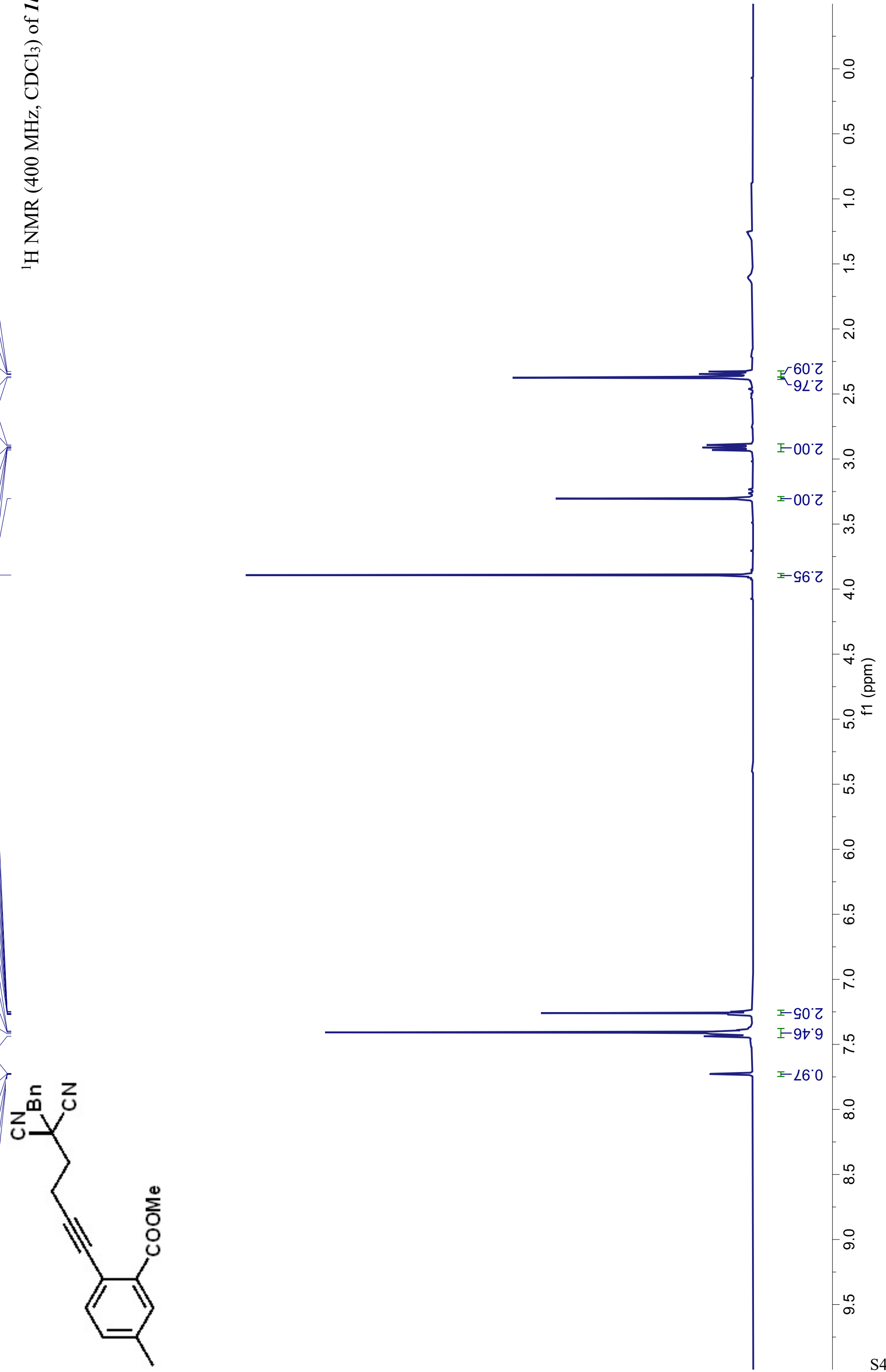


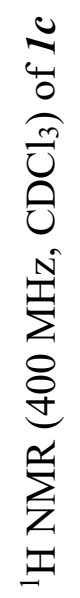

8हع 乙

99ع 乙

$\angle L \mathcal{E}^{\prime} \mathrm{C}^{-}$

$668 \mathrm{Z}$

6เ6'

$6 \varepsilon 6^{\circ} 乙$

๑๐ $\mathcal{E}^{\circ} \varepsilon-$

$8 \angle 8^{\circ} \varepsilon-$

9tr. L

$092 \mathrm{~L}$

$69 \varepsilon^{\circ} L$

$60 t^{\circ} \mathrm{L}$

$028^{\circ} L$
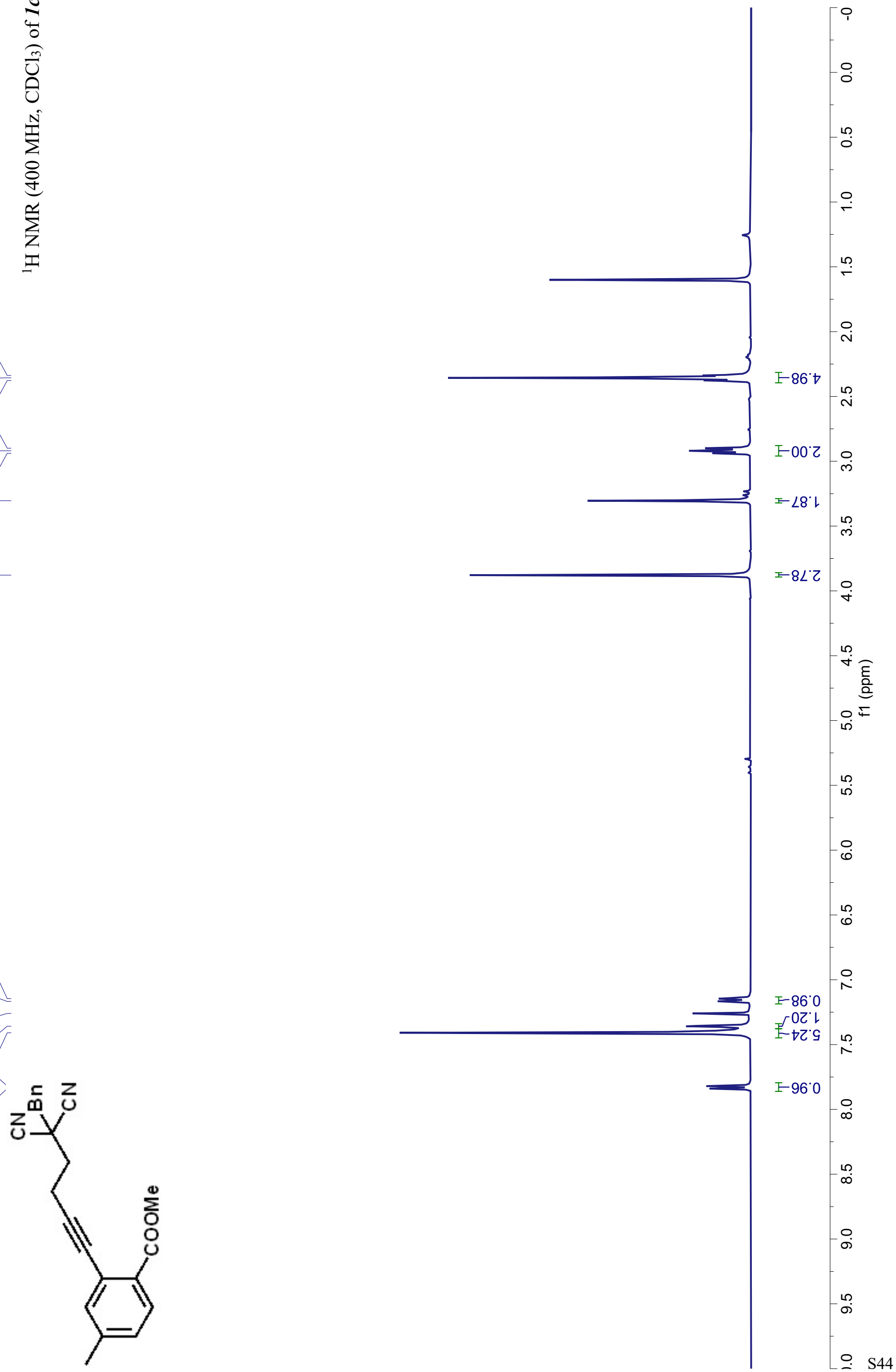


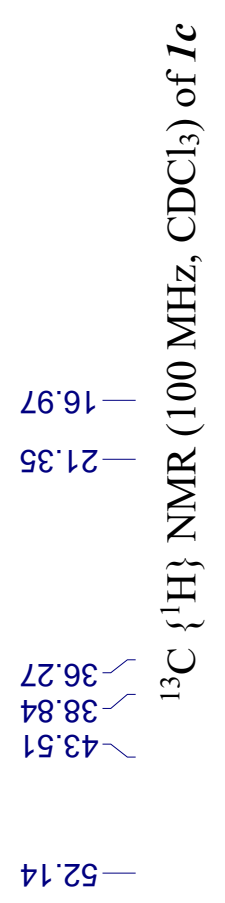




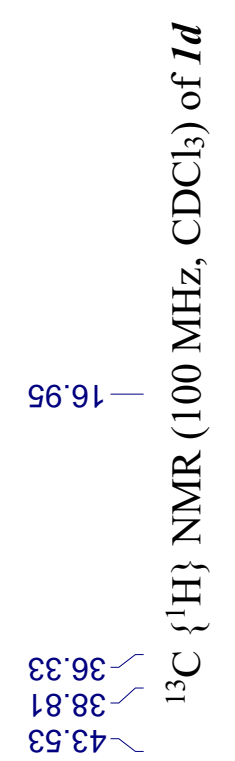

t8. $9 L$

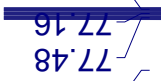

$8 \mathcal{C}^{\circ} \mathrm{L}$

$16.88-$

88・レレ

乙レGレ

乙LGレ

$6 \varepsilon^{\circ} 8$ เ

70 6乙L

$\angle L^{\circ} 62 L$

$\varepsilon \nabla^{\circ} 0 \varepsilon \downarrow$

68'LL

$\neg \varepsilon^{\prime} \varepsilon \varepsilon\llcorner$

99. $9 \varepsilon$ -
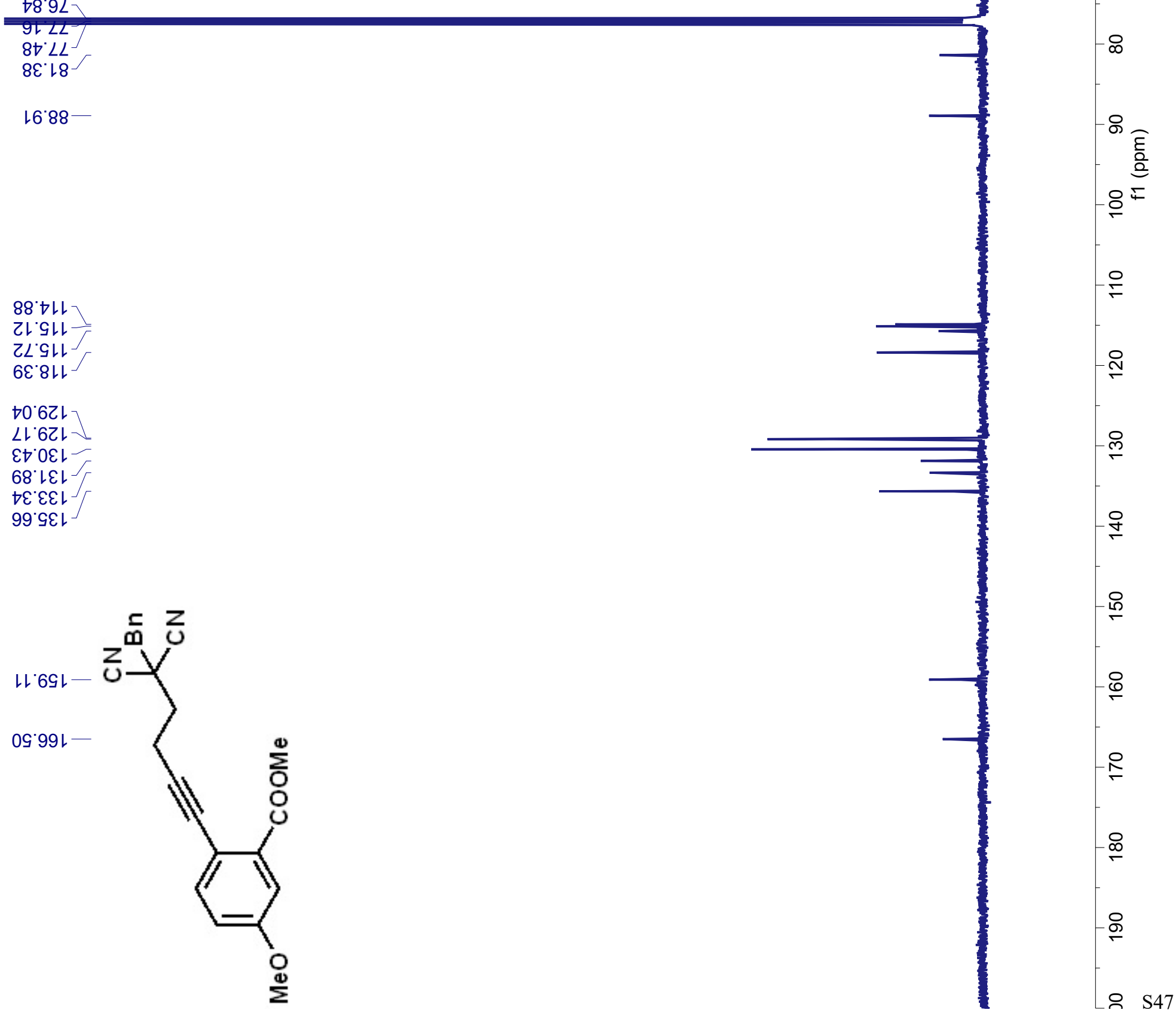

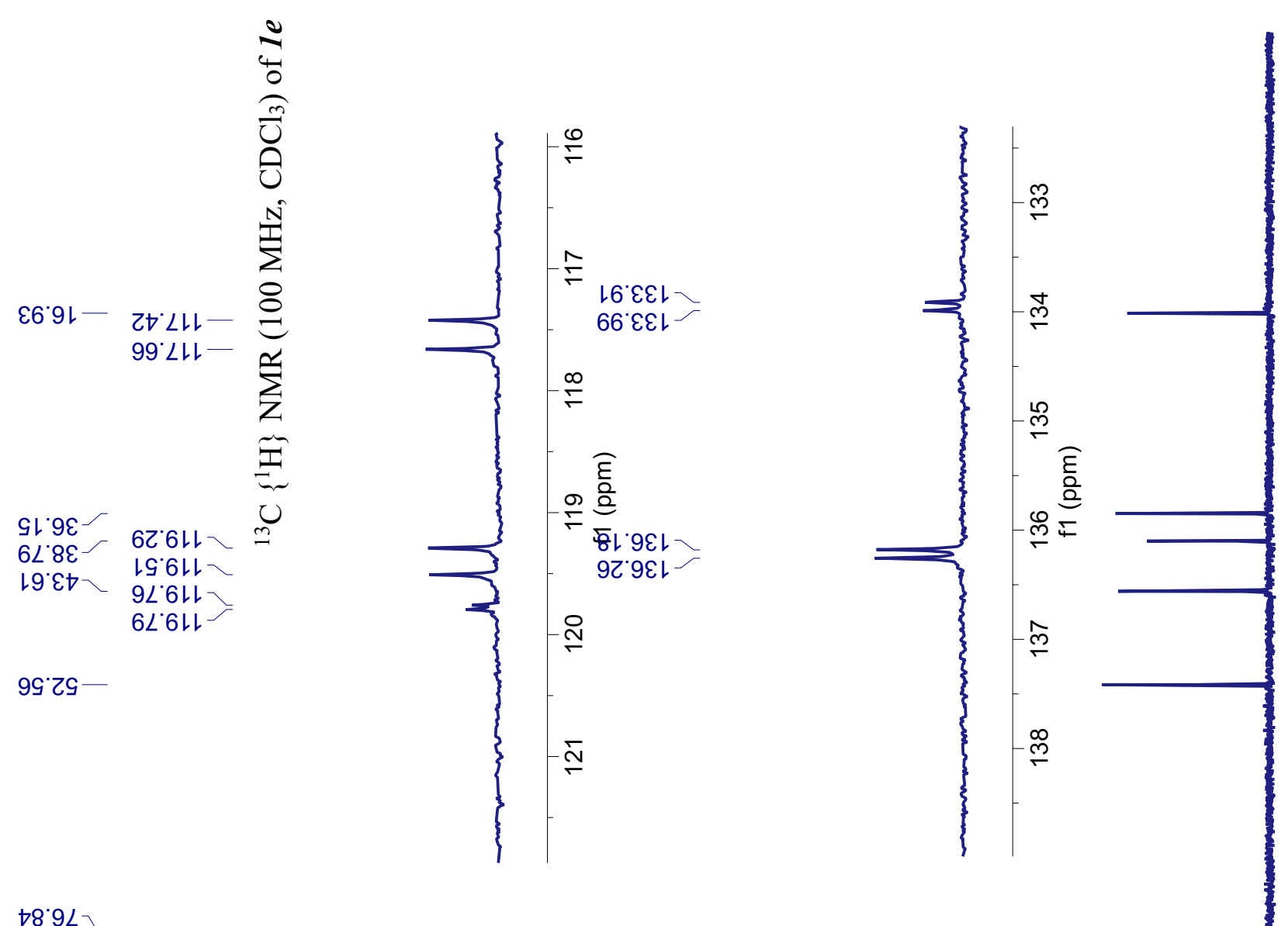

$78 \cdot 9<$

$87^{\circ} \angle L$

19.08

$\angle 9^{\circ} 06$

$69^{\circ} 06^{-}$

†8ヤル

乙大 $\angle L$

$99^{\circ} \mathrm{LLL}$

626

IG'6L

9L6レ

6L6L

606ZL

02 6Zレ

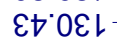

L८LEL

เ 6 हEᄂ

$66^{\circ} \varepsilon \varepsilon\llcorner$

8L' $9 \varepsilon L$

9Z. $9 \varepsilon \downarrow$

乙ナ0 09 -

Z7.09

Lt $99 \mathrm{~L}$

हt 991
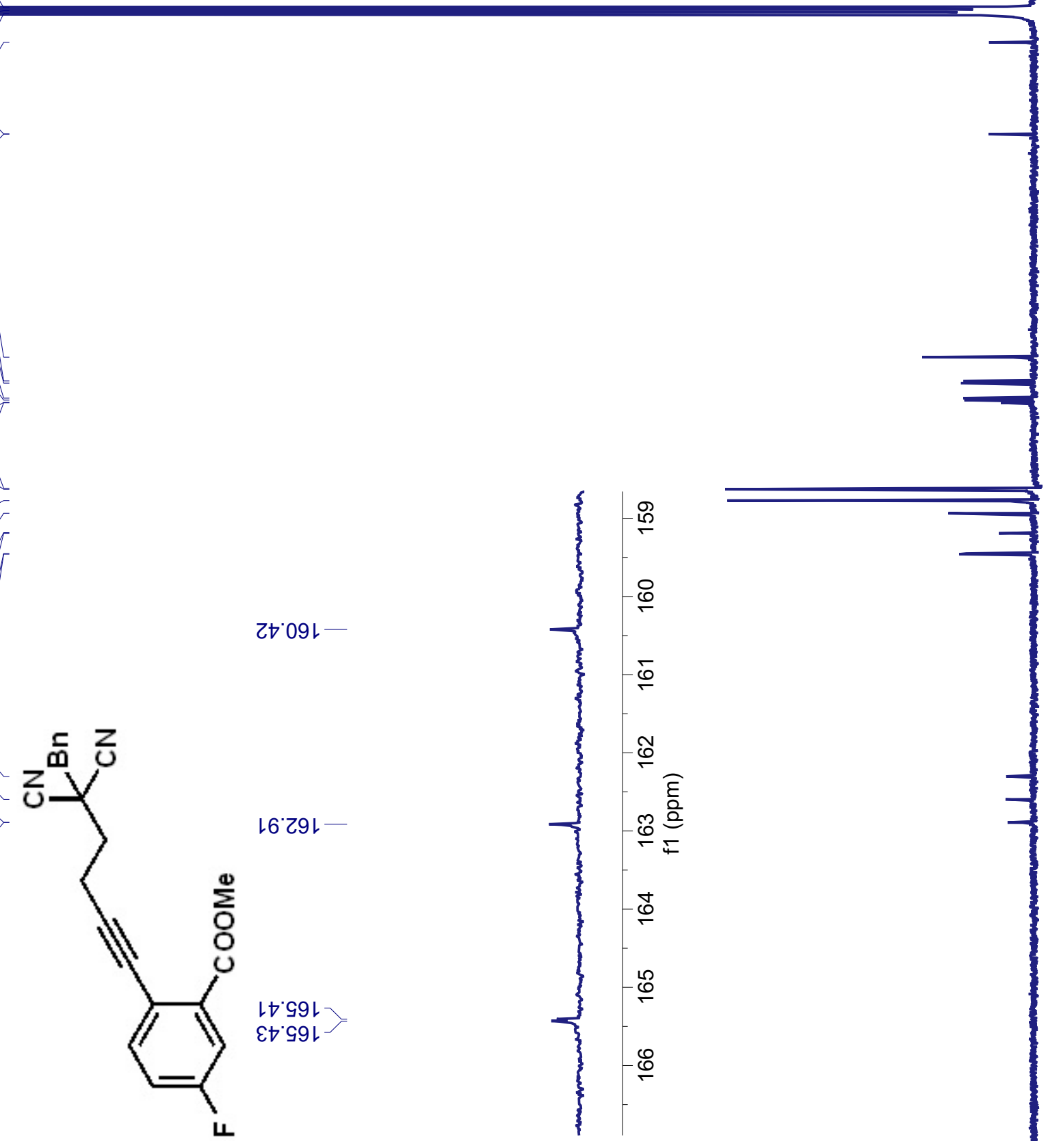

$\infty$

요

$\frac{\bar{\varepsilon}}{2}$
$8^{\frac{2}{2}}$

웉

$\stackrel{\text { ิ }}{\sim}$

$\stackrel{\text { m }}{\stackrel{2}{ }}$

움

-옴

$\stackrel{8}{6}$

옫

$\stackrel{\infty}{\circ}$

옹 


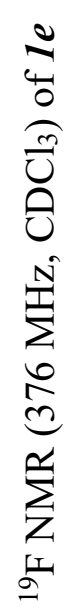

8L9'0ルー-

†99.0

899.0レ-

†G 0 LL-

EtGㅇㄴ-

6E9ㅇㄴ

†ESㅇㄴ-

6เ9ㅇ-

6ISㅇ-
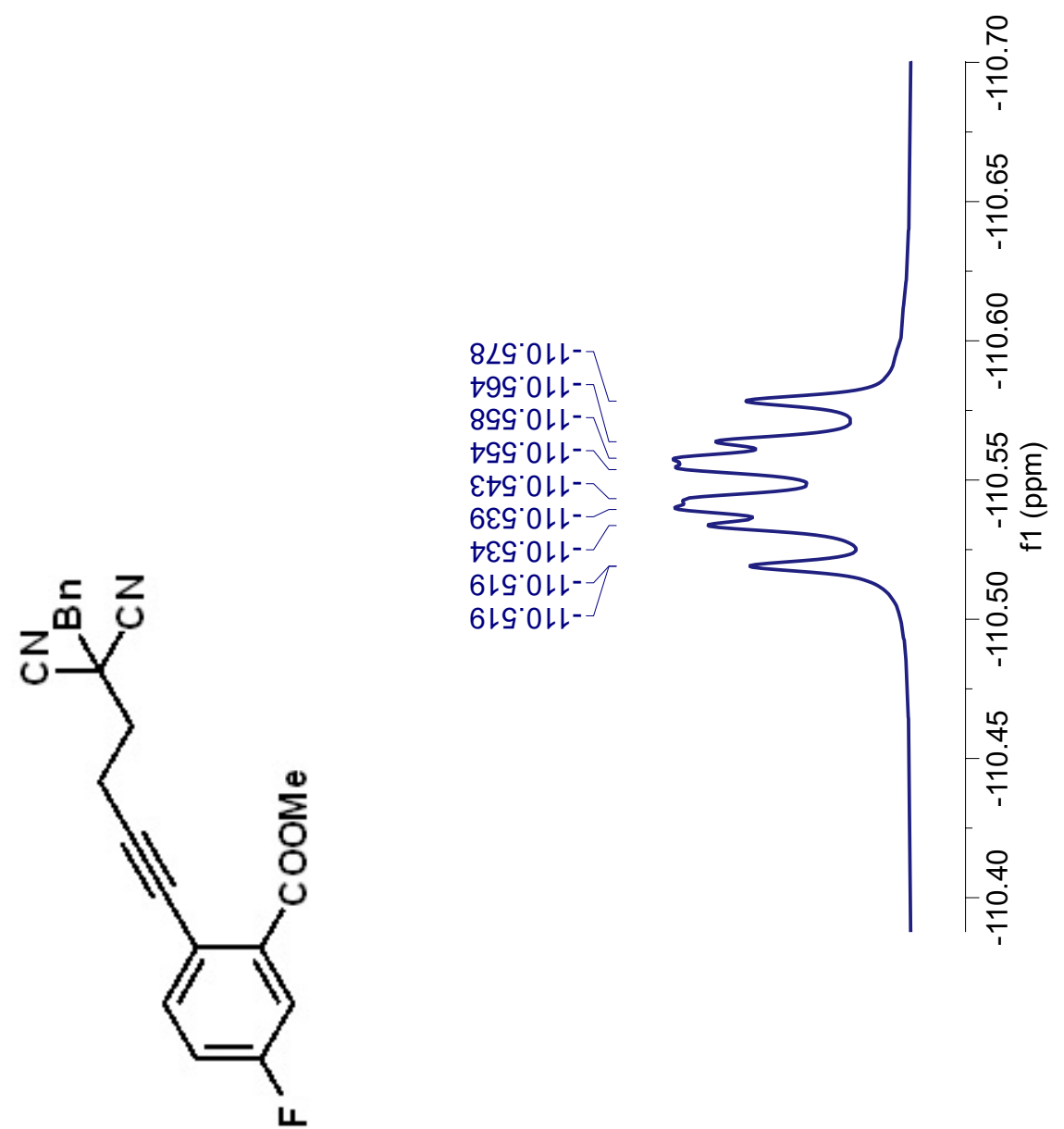

$\infty$

-

8

누

우

오

ำ

$-\frac{0}{1}$

-

-

으 


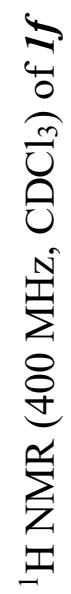

เ乙E'乙

0๑ย'乙-

L68 乙

916'Z-

ง\&6 ${ }^{\circ}$

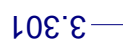

$906^{\circ} \varepsilon-$

$09 Z^{\circ} L$

$06 \varepsilon^{\circ} L$

$900^{\circ} \mathrm{L}$

$60 t^{\circ} \mathrm{L}$

9Zt $L$

ZE० L

$887^{\circ} \mathrm{L}$

$006^{\circ} \mathrm{L}$

$906^{\circ} \mathrm{L}$
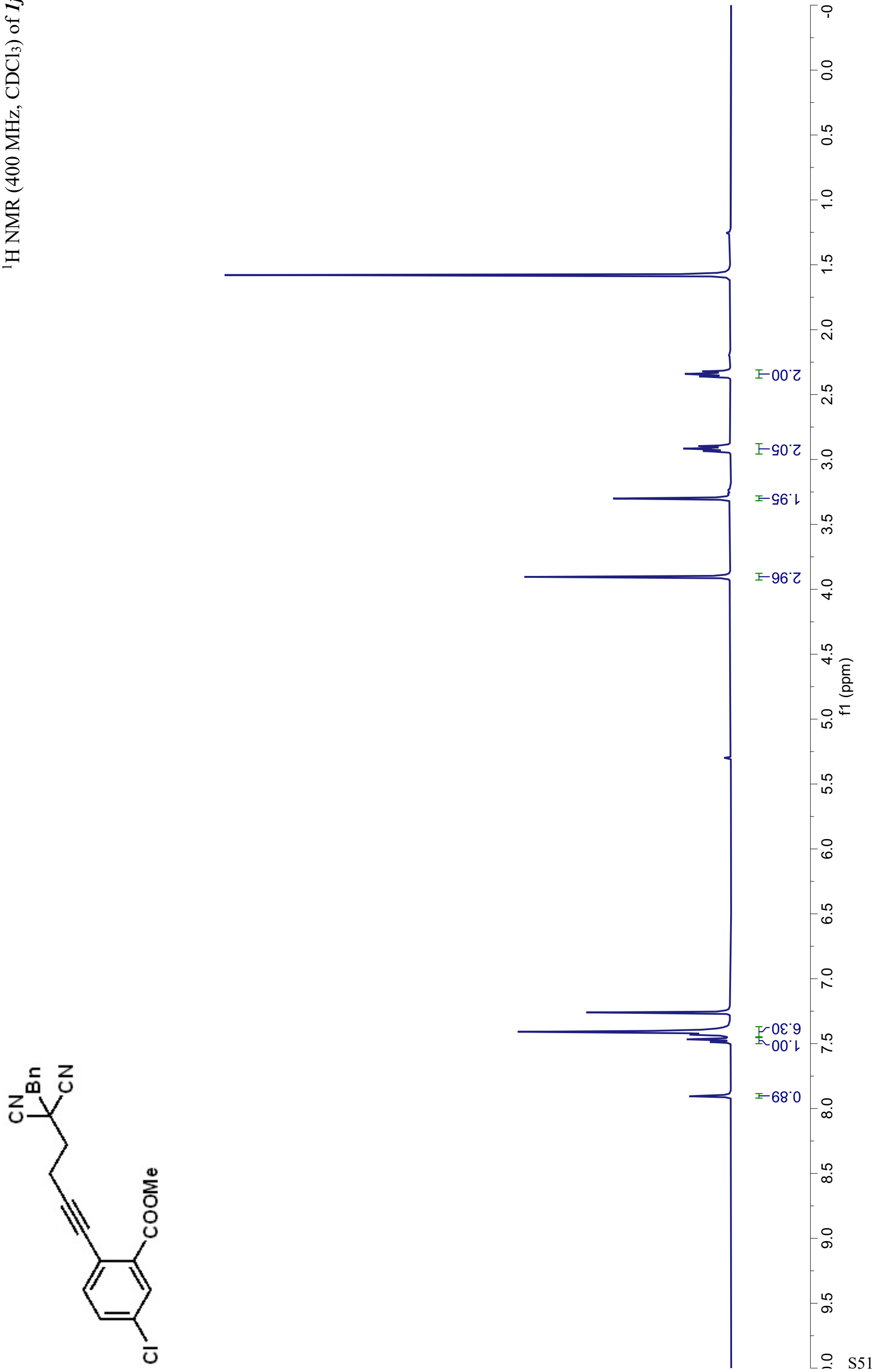


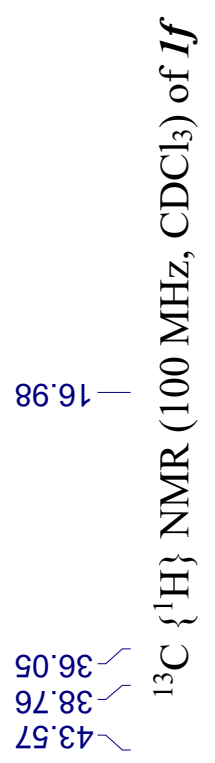

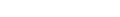

99'29-

†8: $9 L$

$9 L^{\circ} L L J$

$\angle 9^{\circ} 08^{\circ}$

96:เ6-

เ8๋レー

80ててレ -

80 6ZL

6L62L-

ᄂナ0EL

GG $0 \varepsilon L$

$8 L L E L$

G० ZEL

$\varepsilon \varepsilon^{\prime} \varepsilon \varepsilon\llcorner-$

ง० $\nabla \varepsilon\llcorner$

8t $G \varepsilon L$
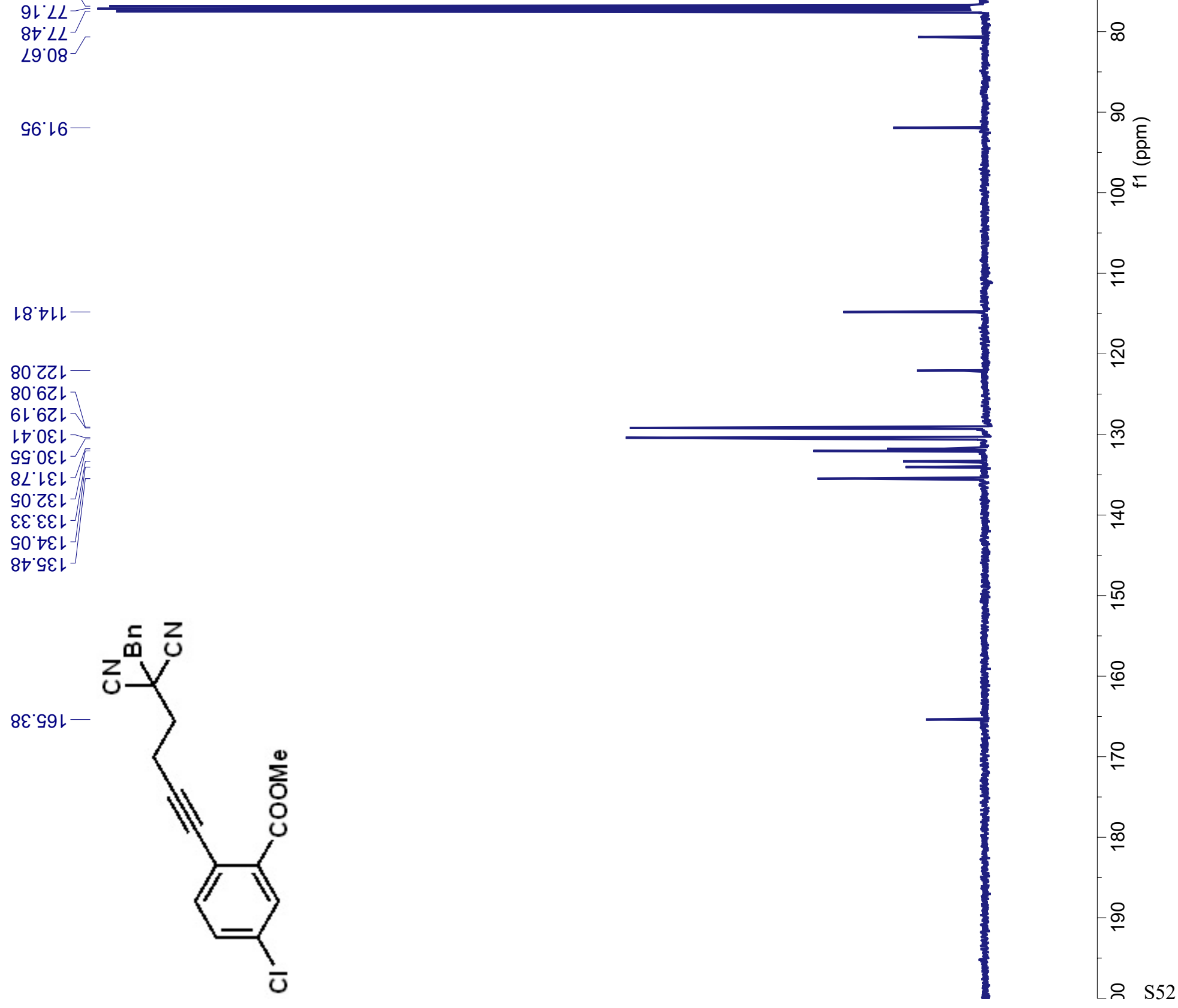


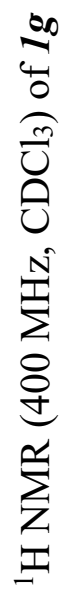

8धย乙

89\& 乙

$\angle L E^{\circ}{ }^{-}$

$186{ }^{\circ} \mathrm{C}$

0962

$0 \angle 6^{\circ} \mathrm{Z}^{-}$

$80 \varepsilon^{\circ} \varepsilon$

$\varepsilon \varepsilon 6^{\circ} \varepsilon-$

692

E9Z $L$

ยเซ L

Z99. $L$

ZL9 $\angle$

$189^{\circ} \mathrm{L}$

$989^{\circ} \mathrm{L}-$

LOL $L$

06! 8 -
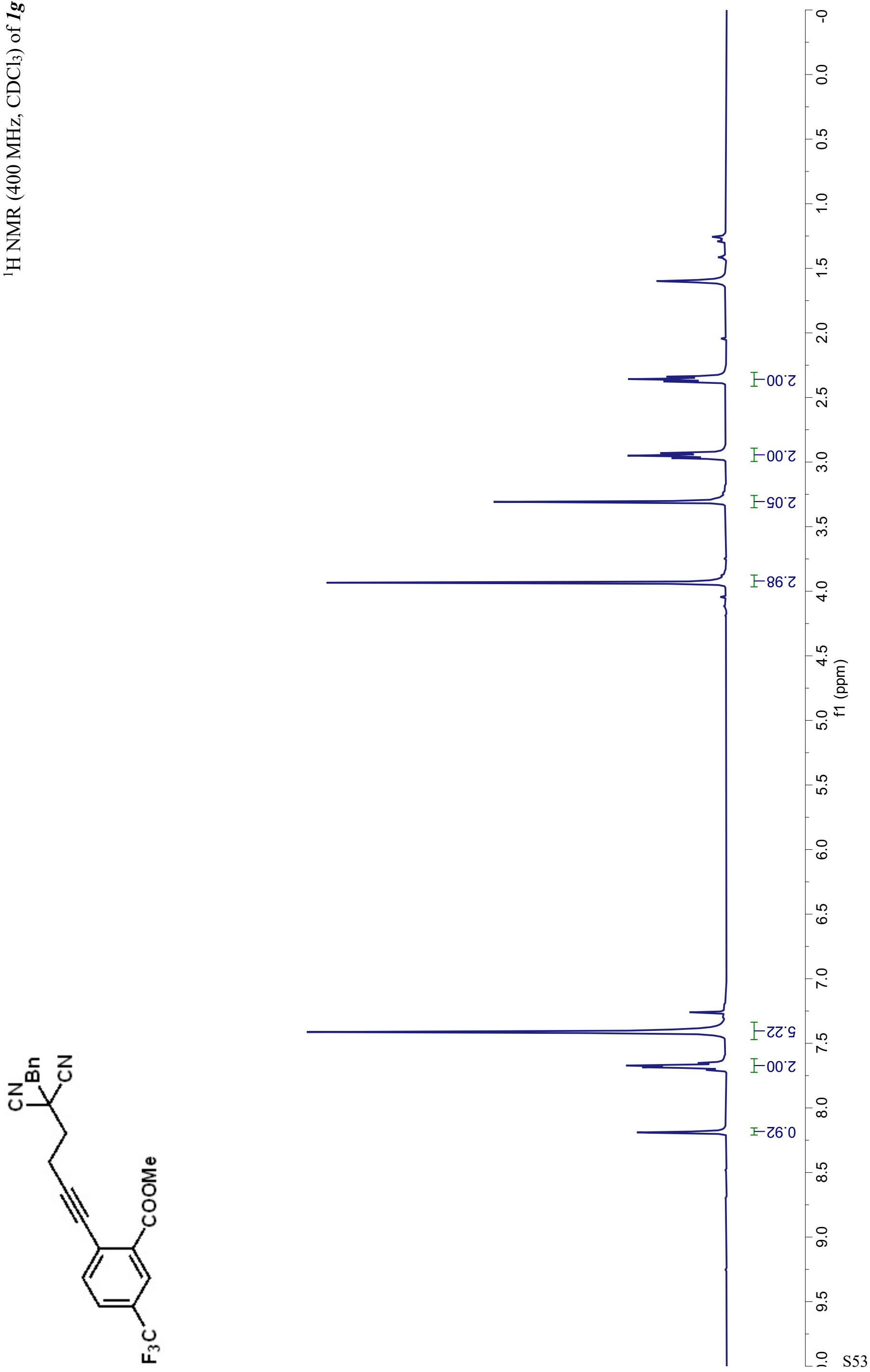


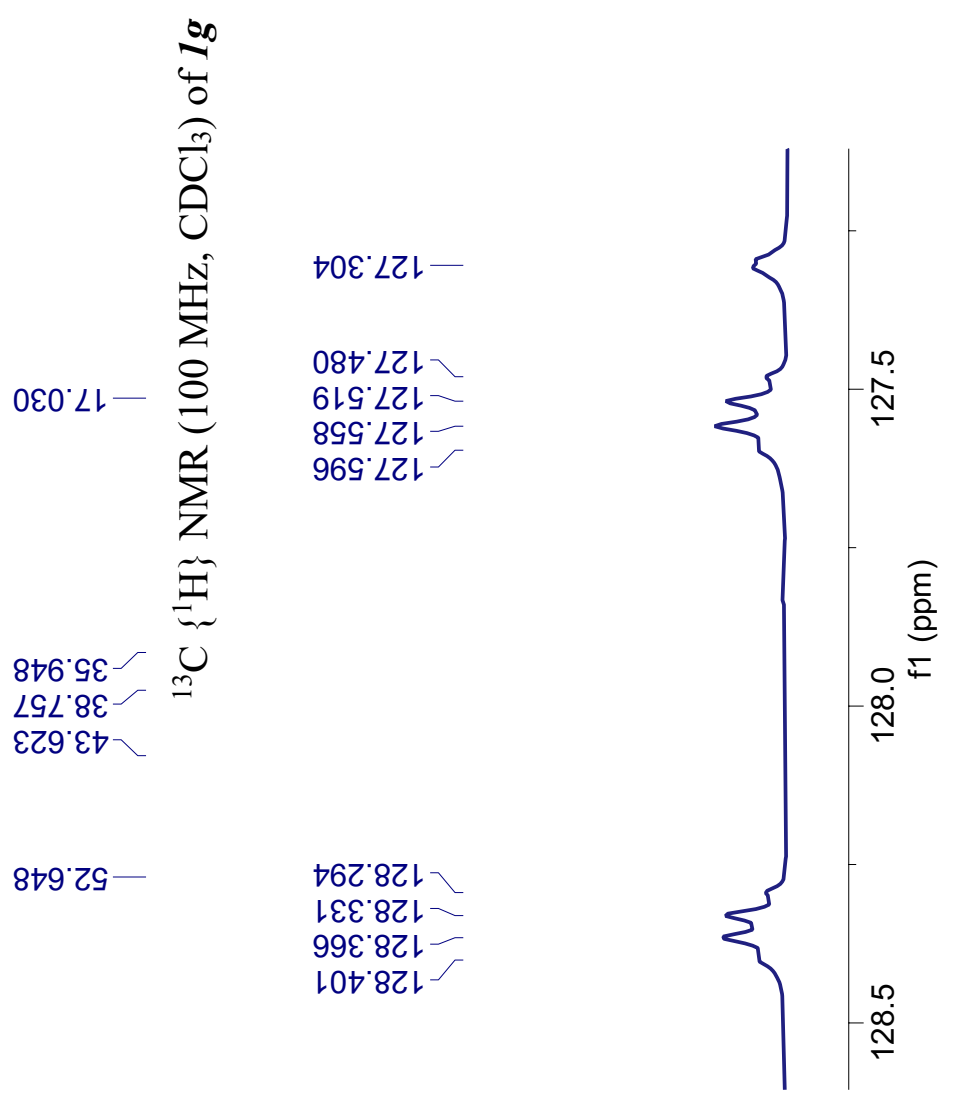

$978.9 L$

791' $\mathrm{LZ}$

$187^{\circ} \angle L$

$\angle \angle 9^{\circ} 08$

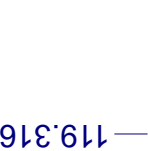

9L6 $86-$

98L゚サレー

9عฑ 6レ

カヤレててレ

๕98 $\bullet 乙 レ$

$\neg 0 \varepsilon^{\circ} \angle Z L$

08t LZL

6IS'LZL

8G9'LZL

969'LZL

†6乙 8ZL

เยE'8ZL

998.8ZL

LOt8ZL

8ルL6Z

乙レで6て

LZ8.6ZL

ZGL'0EL

GE७ $0 \varepsilon L$

$\nabla \varepsilon L \cdot L E L$

OLG'ZEL

$068{ }^{\circ} \nabla \varepsilon L$

LEL'十ZL

Z8L 'LZL

6SE $\angle Z L$

$86 \varepsilon^{\circ} \angle Z L$

8Et LZL -

$G \angle D^{\circ} \angle Z L$

ELL'8ZL

60Z'8ZL

†७乙'8ZL-

08Z 8Z

866 $8 Z$ L

S十0 6ZL

L6062L-

$00 \angle 6 Z$ L

LEO OहL

EG乙 $0 \varepsilon L$

L6Z'OEt

૬૬ع.0ع

$z^{\stackrel{c}{0}}$

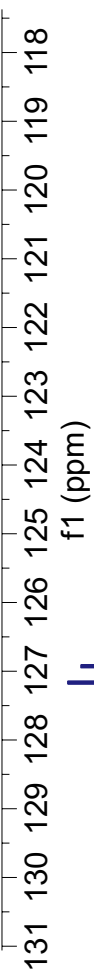

$\infty$

요

흘

으우

운

ํำ

오

$\stackrel{ }{ }$

옴

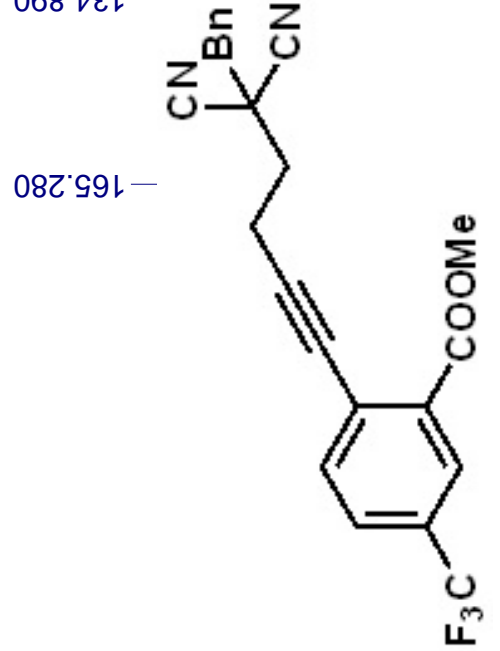

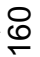

$\stackrel{2}{ }$

$\stackrel{\infty}{=}$

욤 


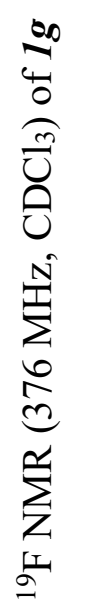

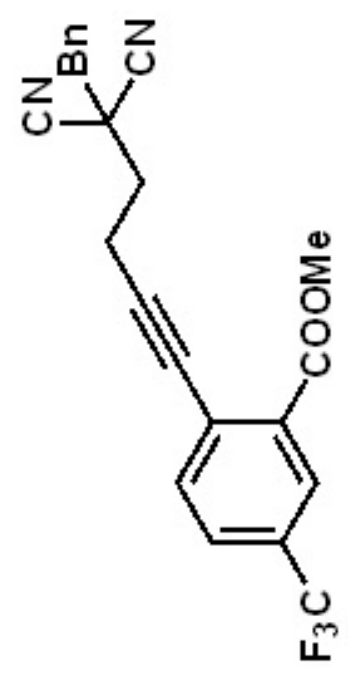




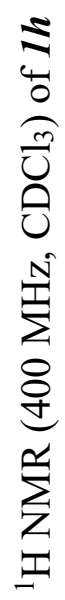

乙†ย'乙

乙9\&'乙-

$18 \varepsilon^{\circ} 乙$

1862

$096^{\circ} \mathrm{Z}$

$0 \angle 6^{\circ} \mathrm{C}^{\circ}$

$90 \varepsilon^{\circ} \varepsilon$

$926^{\circ} \varepsilon$

8\&6 $\varepsilon$

092 $L$

$\neg \angle E^{\circ} L$

$68 \varepsilon^{\circ} L$

ธิ

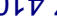

209.

$279^{\circ} \mathrm{L}$

180.8

980.8

$201^{-} 8^{-}$

9018

9L9'8-
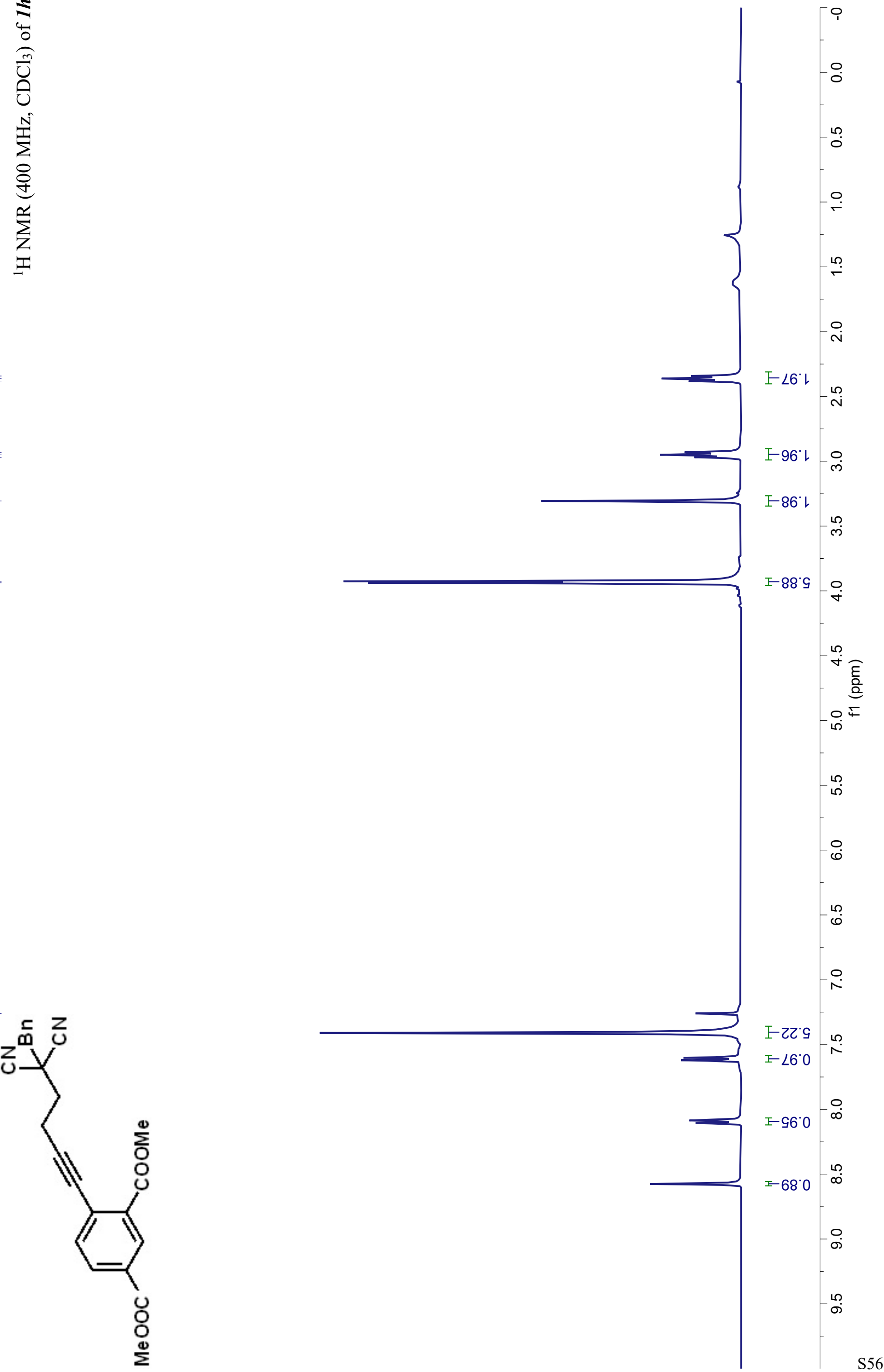


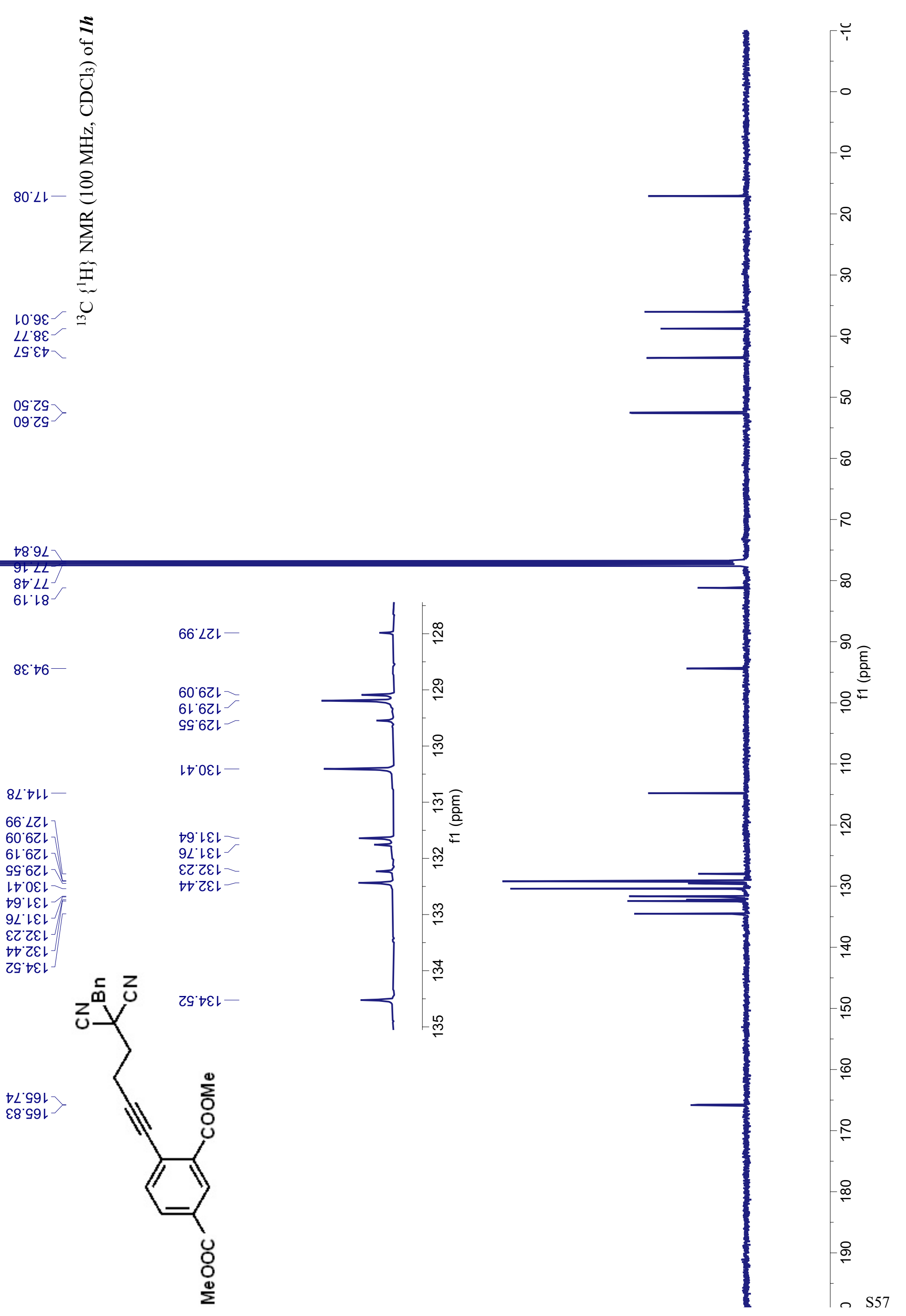




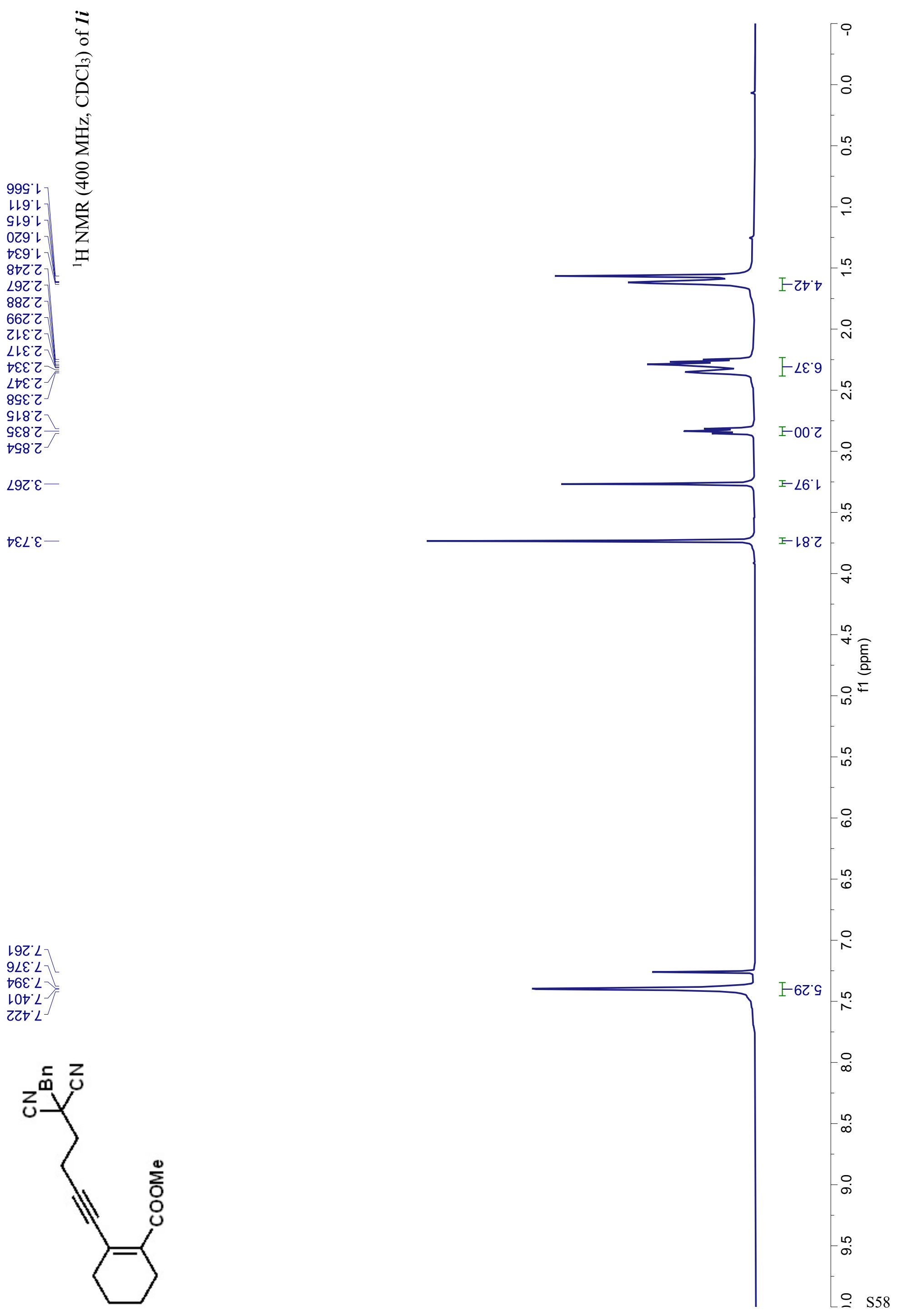


$70 \angle L-Ð$

$\varepsilon<\cdot L Z>$

18.12
$62.92-\sum$

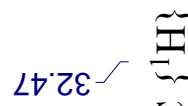

$\angle Z 9 E-U^{\prime}$

$08^{\circ} 8 \varepsilon$

IS' $\varepsilon$

LLIS-

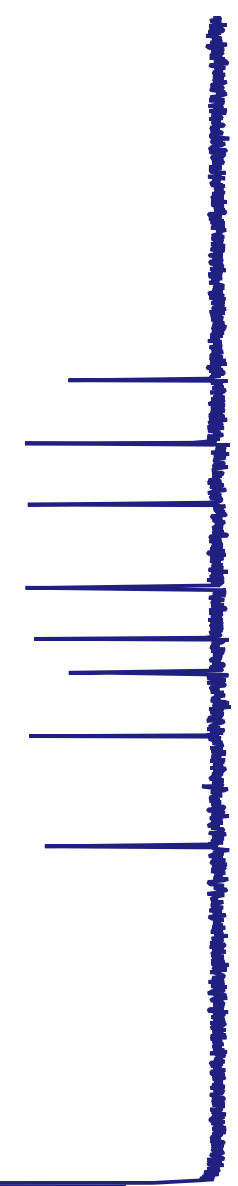

으

우

요

우

-요

8

웃

$78.9 L$

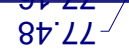

เ० $88-$

เ6. 26

08・ルーー

98.8ZL

$90^{\circ} 6 Z \mathrm{~L}$

$8 L^{\circ} 6 \mathrm{LL}$

เT $0 \varepsilon \downarrow$

S8'LEL

O๋๋ $\downarrow \varepsilon$
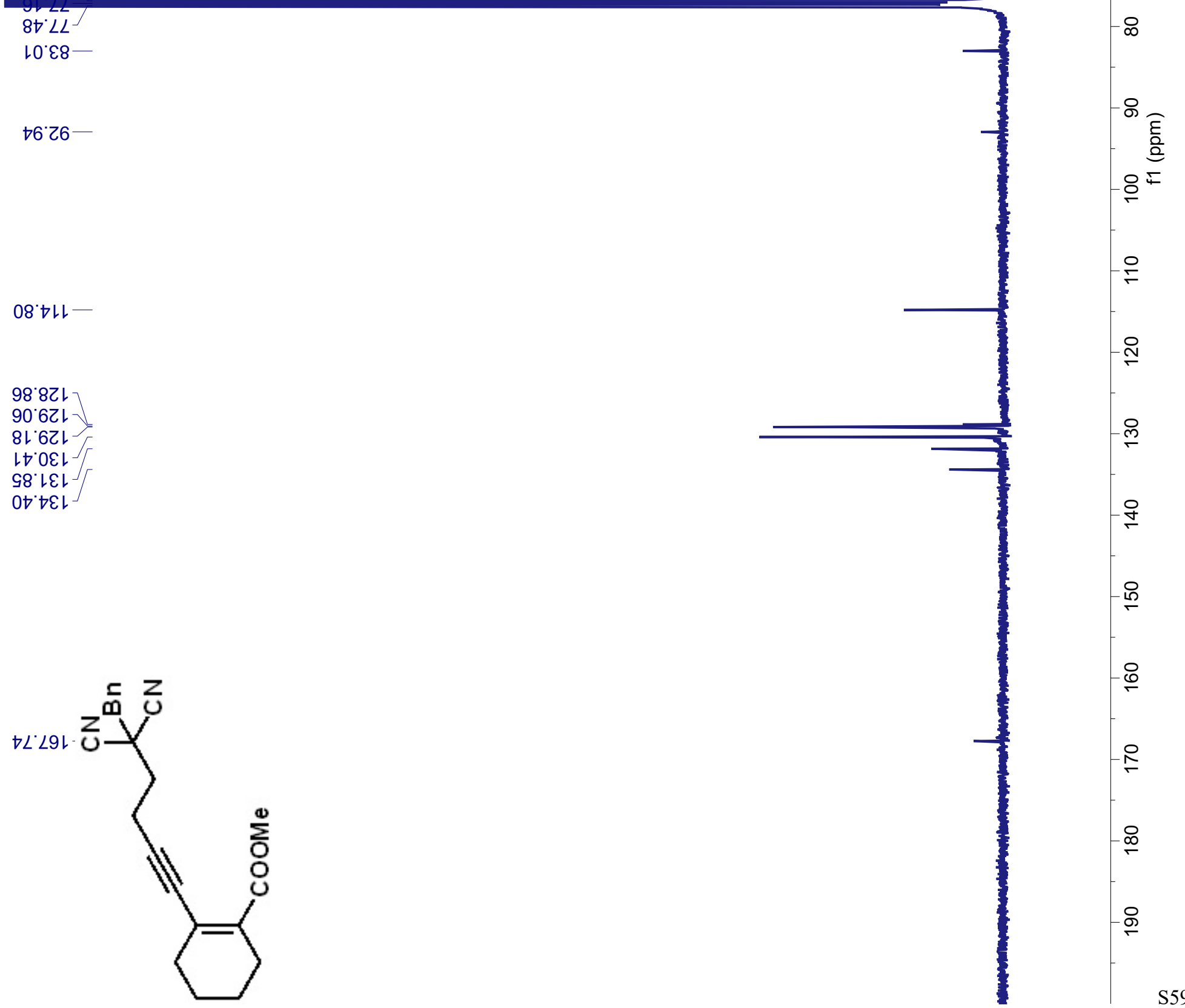


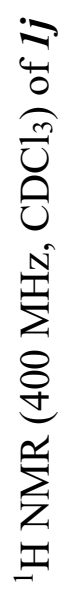

S68' -

๕乙ย'

งฤย'て

乙9ย'乙

$298^{\circ} \mathrm{Z}$

$188^{\circ} \mathrm{Z}$

$106{ }^{\circ}$

$\angle L 6^{\circ} \varepsilon-$

$6 \varepsilon \varepsilon^{\circ} L$

$\angle G \mathcal{E}^{\circ} \angle$

$\angle L E^{\circ} L$

$8 \varepsilon t^{\circ} L$

8St $L$

$9 \angle t^{\circ} L$

GES L $L$

ESG $L$

IL6 $\mathrm{L}$

LE6 $L$
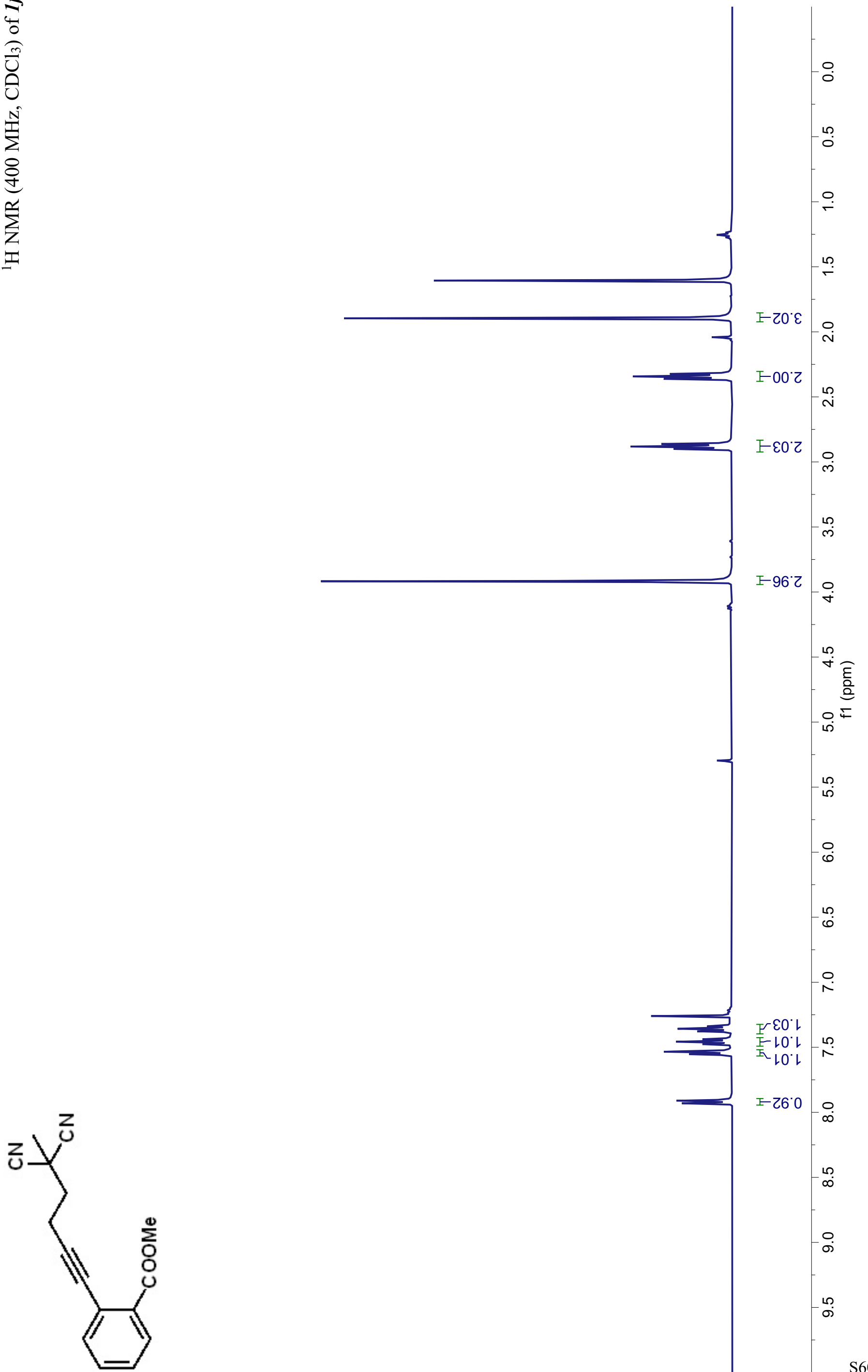


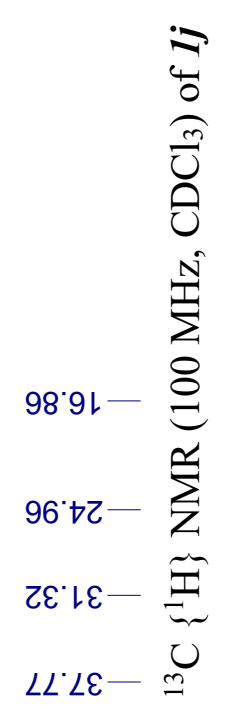

乙ย'ฉ९-

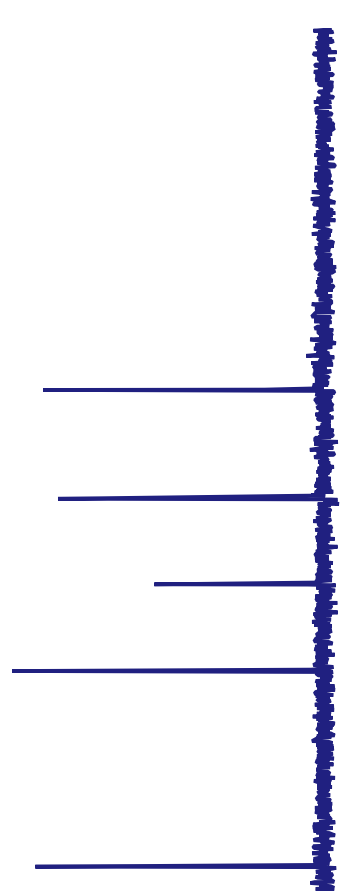

$78.9 L$

9.LLJ

$99^{\circ} 18$

LLO06-

SL・Gルー

69'ย乙レ

ह0.8ZL

† $0 \varepsilon L$

L6. LE

$\angle 6 \cdot L L$

$8 \varepsilon^{\circ} \downarrow \varepsilon\llcorner$
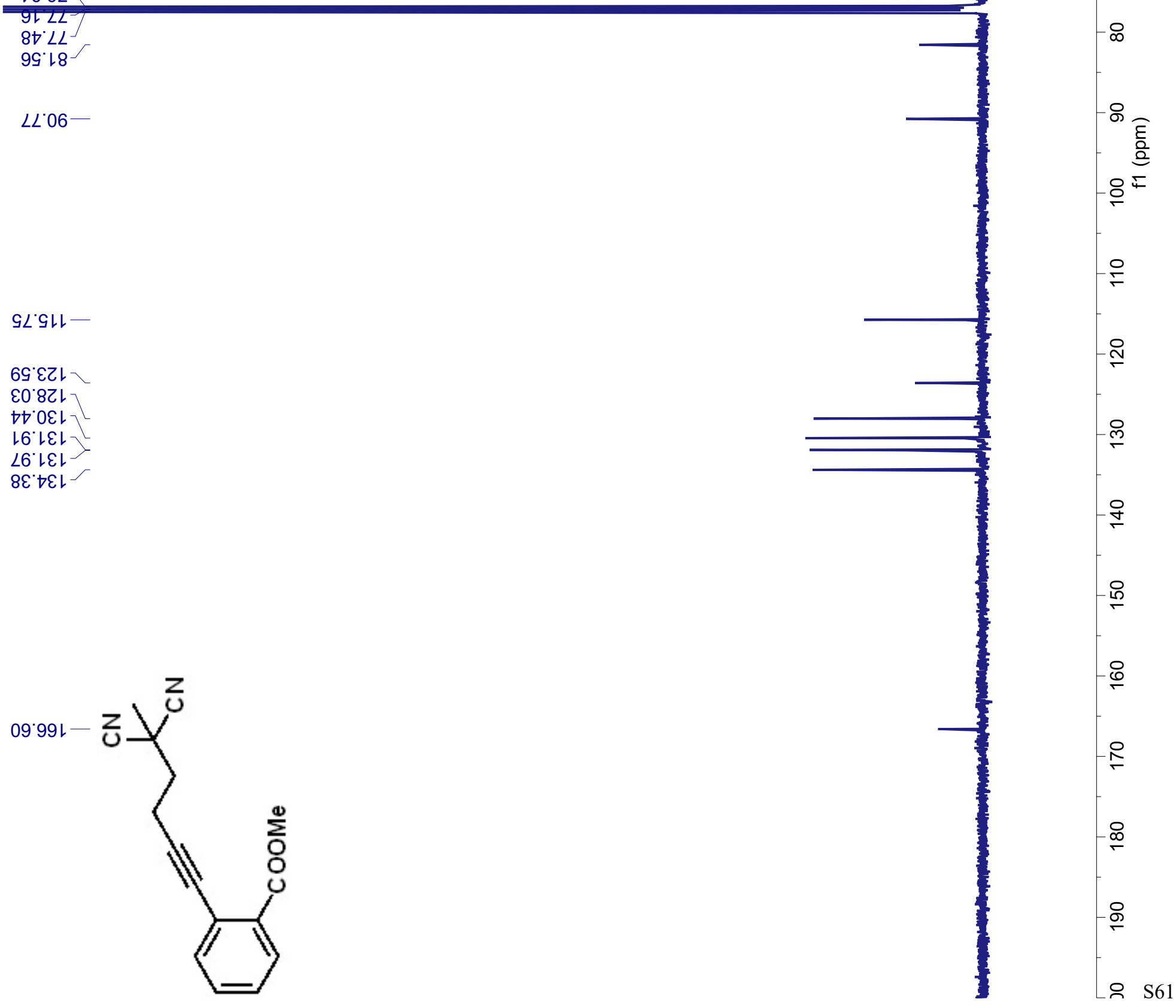


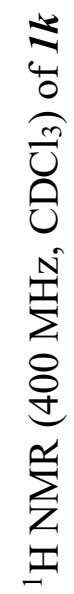

1092

$\varepsilon 09^{\circ} \mathrm{Z}$

เ19 2

$619 \mathrm{C}$

ZZ9'

$\angle 29{ }^{\circ} \mathrm{Z}$

५\&9 Z

$1+9 \cdot 2$

$\angle 9 L$ 乙

$\nabla L L ' Z$

$18 L 2$

$\angle 8 L Z$

$06 \angle Z$

908 $\mathrm{Z}$

$808^{\circ} \mathrm{Z}$

$868^{\circ} \varepsilon$

092 $L$

$\checkmark Z \varepsilon^{\circ} L$

$\angle Z E^{\prime} \angle$

$\varepsilon \succsim \varepsilon^{\circ} L$

$9 \nabla \varepsilon^{\circ} L$

乙9\& $\angle$

G $9 \varepsilon^{\circ} L$

6เt L

ย乙ち L

8Et $L$

Zt大 $L$

$\angle G D^{\circ} \angle$

$19 t^{\circ} \mathrm{L}$

$\varepsilon \angle t^{\circ} L$

$9 \angle \nabla^{\circ} L$

$180^{\circ} \mathrm{L}$

$\angle 87^{\circ} \angle$

$167^{\circ} \mathrm{L}$

96t $\mathrm{L}$

LOS' $\mathrm{L}$

$809^{\circ} \mathrm{L}$

GIS'L

IZG' $L$

9Z9' $L$

$0 \varepsilon S^{\circ} L$

EES $\angle$

$\angle E G^{\circ} L$

$689^{\circ} \mathrm{L}$

$969^{\circ} L$

$009^{\circ} \mathrm{L}$

$709^{\circ} \mathrm{L}$

II ${ }^{\circ} \mathrm{L}$

$919^{\circ} \mathrm{L}$

$029 \angle$

G68. $\mathrm{L}$

$668^{\circ} \mathrm{L}$

SL6. $\mathrm{L}$

눙

$616^{\circ} \mathrm{L}$
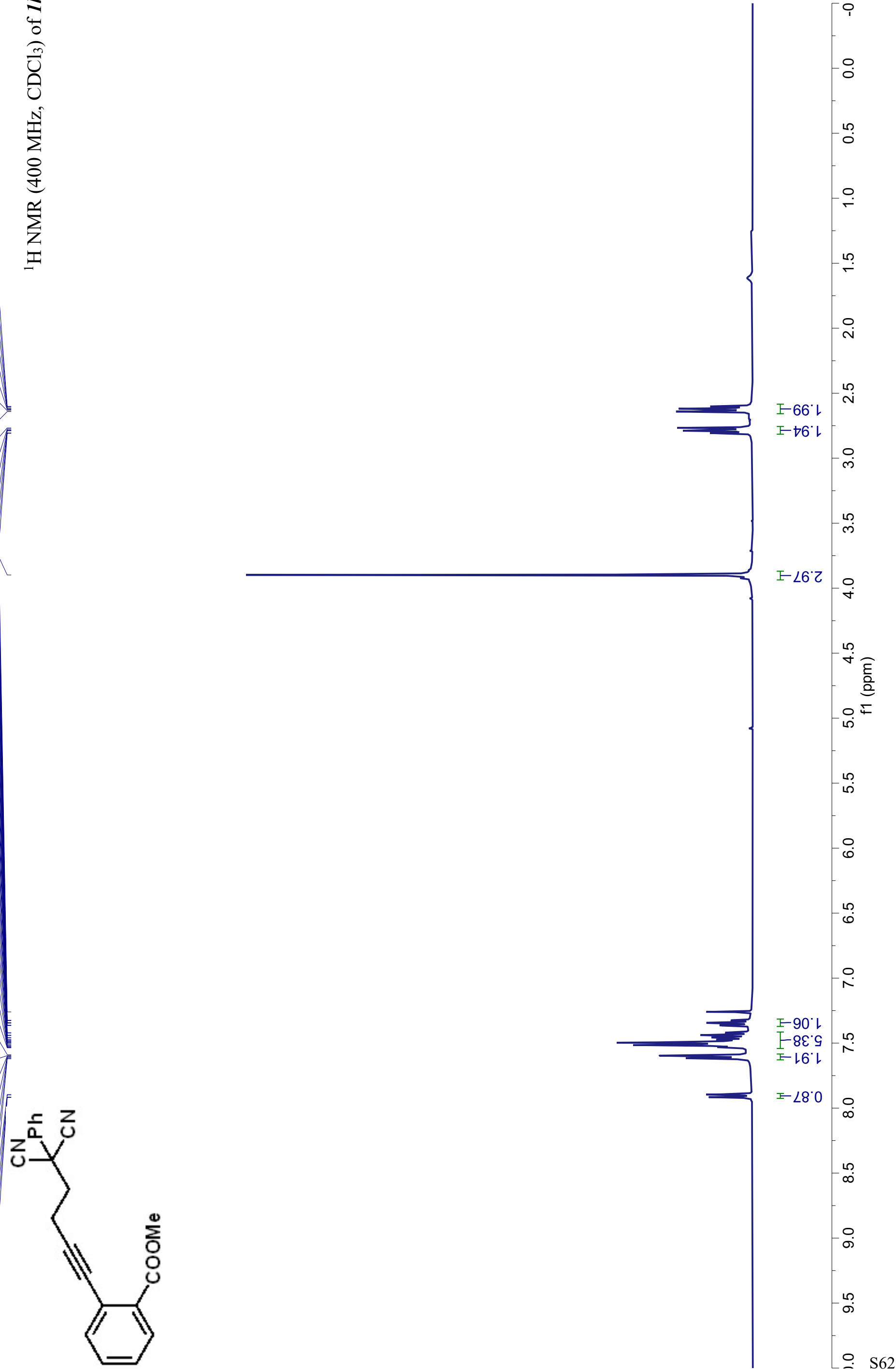
s8.91-

E0.เ
S9.เ

เฉ'
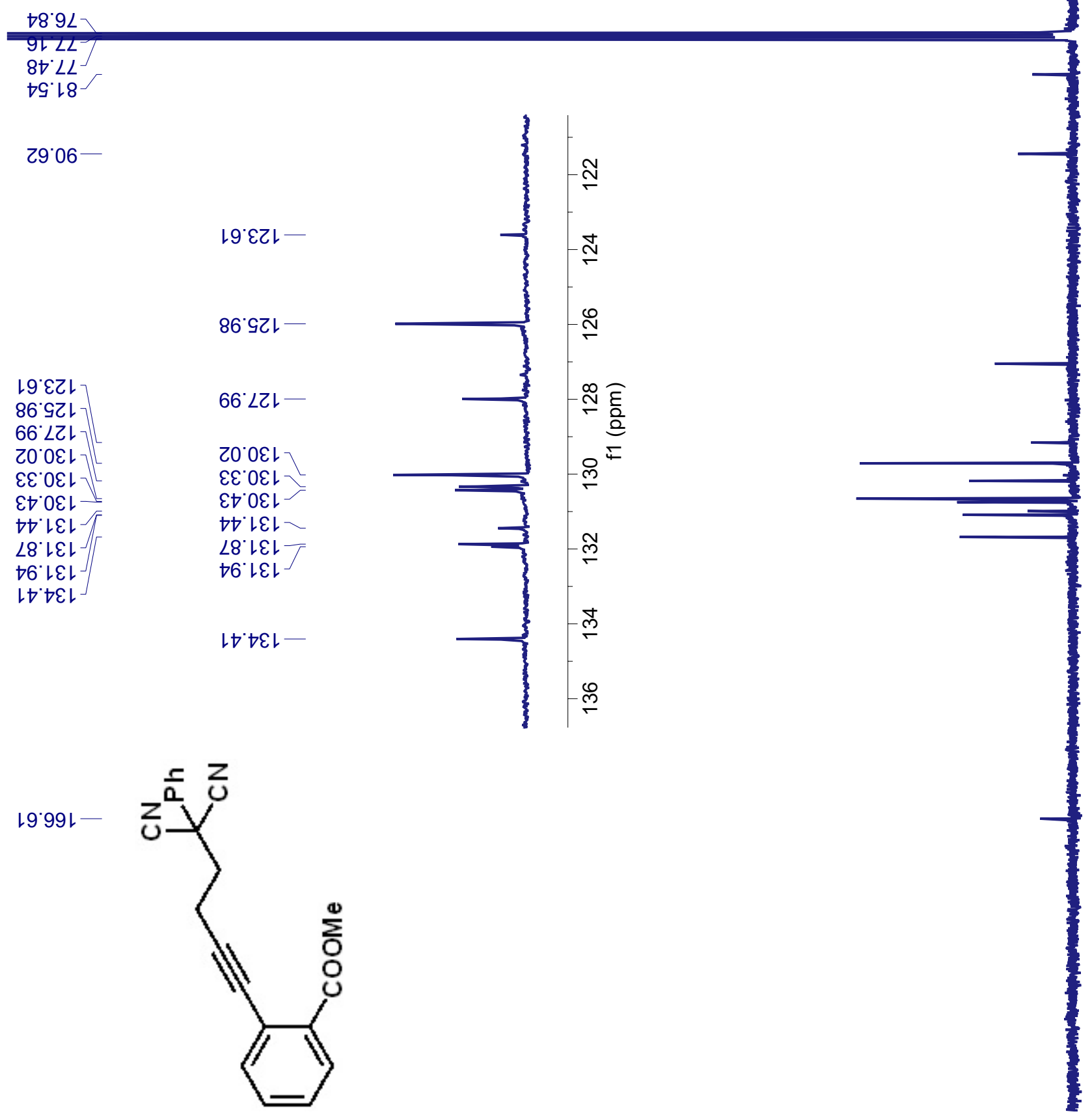

$\infty$

- 요

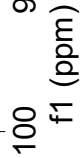

을

ํㅗ

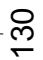

움

옴

8

$-6$

온

$\stackrel{\infty}{-}$

욤 
$906^{\circ} \mathrm{L}$

$806^{\circ} \mathrm{L}$ SZ6 $\mathrm{L}$ 826.2

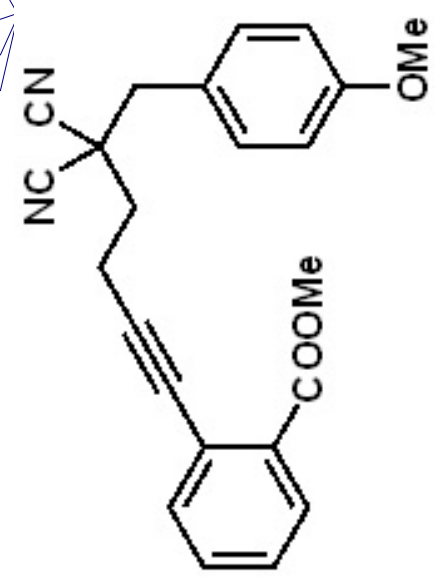




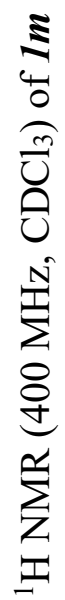

$\angle L E^{\prime} Z$

$\angle Z \varepsilon^{\prime} Z$

$\nabla \varepsilon \varepsilon ' 乙$

$9 \varepsilon \varepsilon^{\circ} Z$

$0 \downarrow \varepsilon ' Z$

$\angle \forall \varepsilon^{\prime} Z$

$99 \varepsilon^{\circ} Z$

$S \angle \varepsilon^{\prime} Z$

$888^{\circ} \mathrm{Z}$

$\angle 68^{\circ} \mathrm{Z}$

†06 $\mathrm{Z}$

$806^{\circ} \mathrm{Z}$

$\angle 16{ }^{\circ} \mathrm{Z}$

$\angle Z 6^{\circ} Z^{\circ}$

$\varepsilon \varepsilon \varepsilon^{-}$

$606^{\circ} \varepsilon-$

$10 t^{\circ} 9$

$90 t^{\circ} 9$

ott. 9

เレナ9

$0 \angle t \cdot 9$

ZLt' 9

8Lt'9-

$087^{\circ} 9$

$09 Z^{\circ} L$

$\neg \varepsilon \varepsilon^{\circ} L$

$8 \varepsilon \varepsilon^{\circ} L$

$\varepsilon \subseteq \varepsilon^{\circ} L$

$\angle S \varepsilon^{\circ} L$

$\varepsilon L \varepsilon^{\circ} L$

$9 \angle E^{\circ} \angle$

LEt $L$

SEt $L$

LGt $\angle$

$\forall G t^{\circ}<$

$9 S t^{\circ} L$

$69 t^{\circ} L$

$19 t^{\circ} L$

$69 t^{\circ} \angle 7$

$\varepsilon \angle t^{\circ} L$

$\angle E S^{\circ} \angle$

$0 t S^{\circ} L$

9SG $L$

$099^{\circ} \mathrm{L}$

$906^{\circ} \mathrm{L}$

606.

926.

$6266^{\circ} \mathrm{L}$
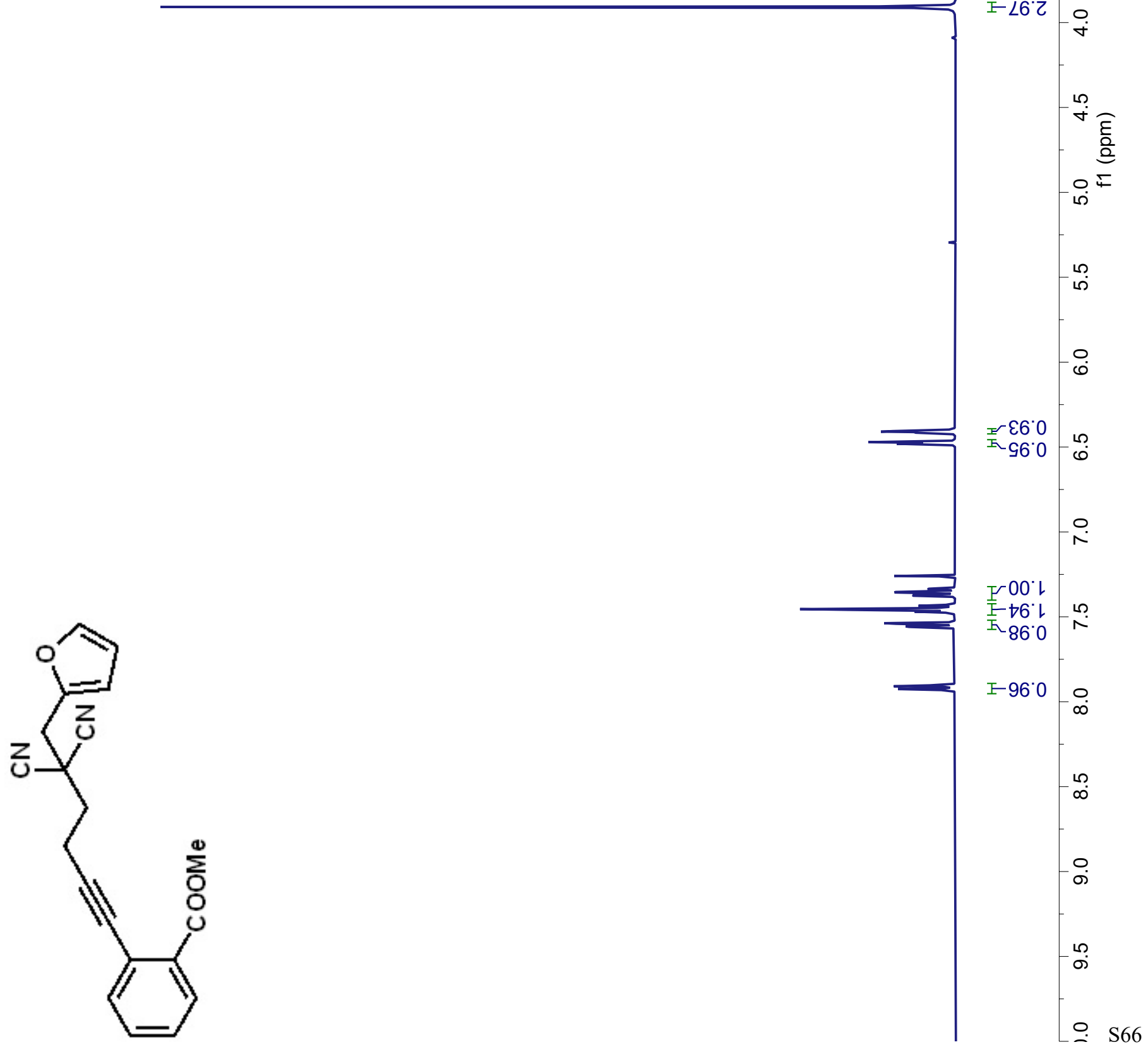
$88.91-\underbrace{\sum_{0}^{ \pm}}$

$8 Z^{\circ} \mathrm{ZG}$

t8. $9 L$

$9 I^{\circ} L L-$

$87^{\circ} \angle L$

$19^{\circ} 18^{-}$

$\varepsilon L \cdot 06-$

ELเUL

8L.ᄂL

เ9เルL

$\varepsilon \mathcal{~}^{\circ} \varepsilon\llcorner$

Z0.8ZL

$\varepsilon \rightarrow 0 \varepsilon L$

$\angle 8$ LEL

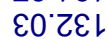

$\angle \varepsilon^{\circ} \downarrow \varepsilon L$

เ8ยทレ -

t0.9tレ
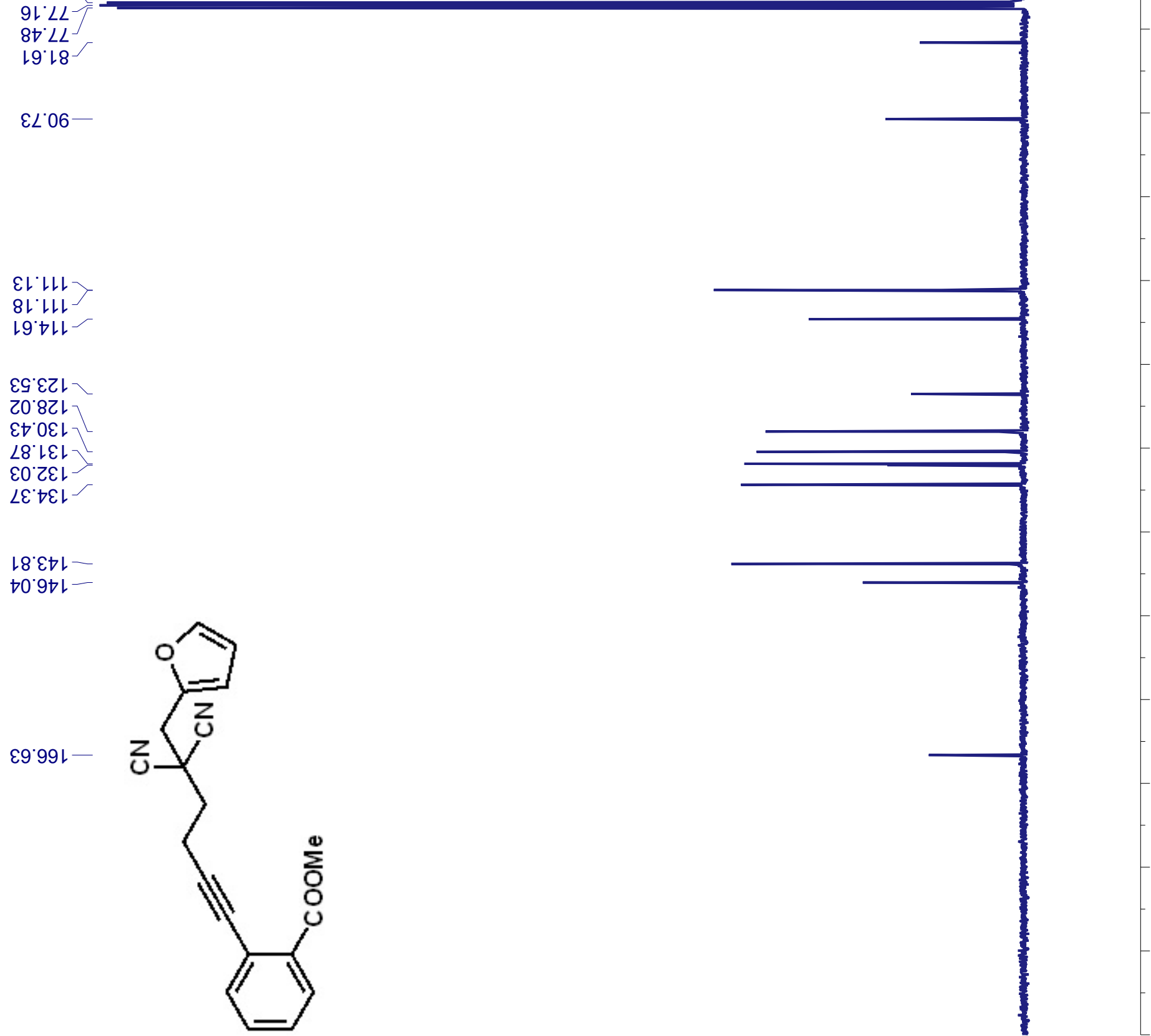

$\infty$

8)

읕

$\stackrel{\text { ำ }}{-}$

오

움

움

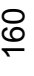

온

$\stackrel{\infty}{\circ}$

옴 


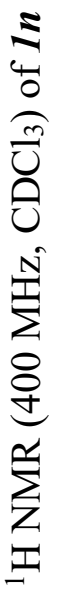

乙Ъ乙

8LE'

乙ยะ'乙

$9 \varepsilon \varepsilon \cdot 乙$

เทย'乙

IGE乙

$99 \varepsilon$ '

L $\angle \mathcal{} \cdot Z$

$898^{\circ} \mathrm{Z}$

$\downarrow \angle 8^{\circ} \mathrm{Z}$

E68 $乙-$

$\angle 06^{\circ} Z^{-}$

2L6

$916^{\circ} \varepsilon-$

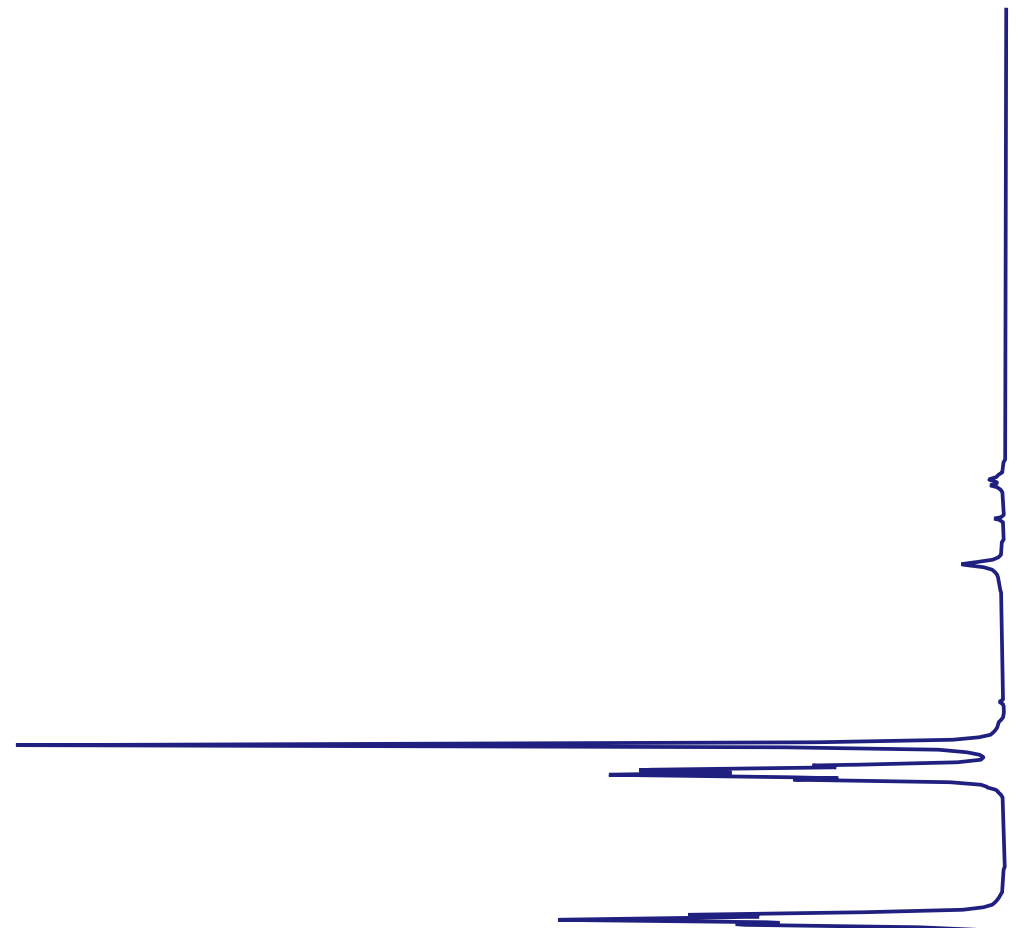

\section{8}

$09 Z^{\prime} L$

$8 \varepsilon \varepsilon^{\circ} L$

$\angle G \mathcal{L}^{\circ} \angle$

$\angle L E^{\circ} \angle$

0 0t 2

8St'L

$8 \angle t \angle$

OtG' $L$

$6 S^{\circ} L$
LG $L$

IE6 $L$

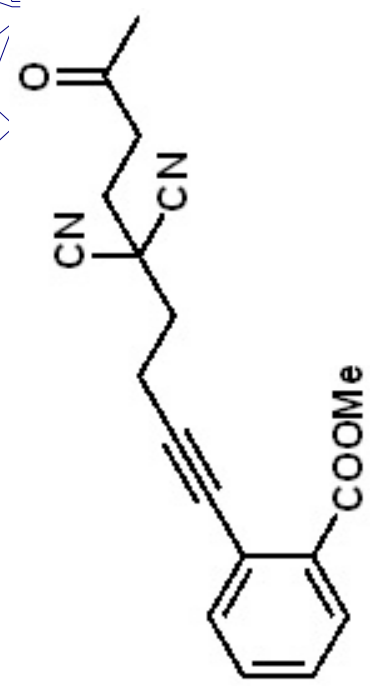




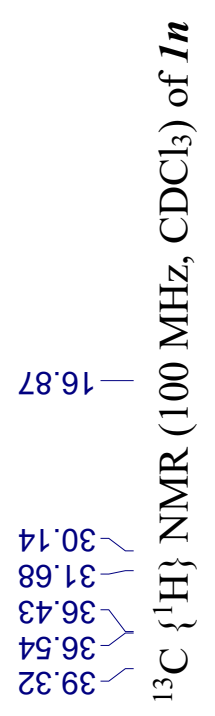

LE'ZS-

$\succ 8 \cdot 9 L$

$9 L^{\circ} L L$

$87^{\circ} \angle L$

$0 L^{\circ} 18$

$29^{\circ} 06-$

6ட゚サルー

† 'E乙

S0.8ZL

七๐०عL

06. LEL

66 เEL

$8 \varepsilon^{\prime} \nabla \varepsilon L$
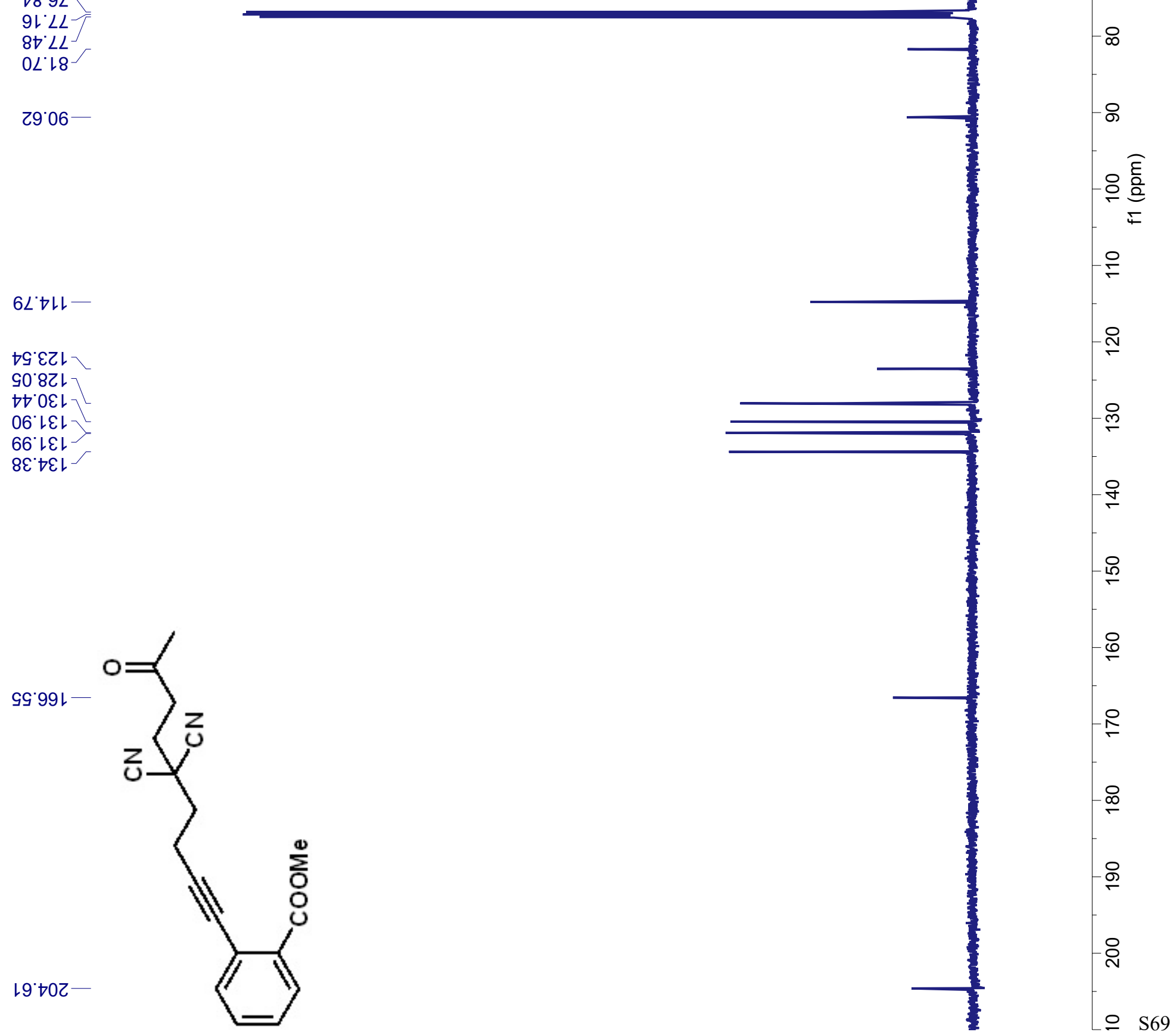


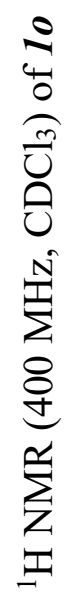

$\varepsilon 6 \varepsilon^{\prime} Z_{7}$

แเ๋

6Zt 2

$\nabla \nabla G \cdot Z$

E99' 2

$799^{\circ} Z$

$789^{\circ} Z$

S6L' 2

$\angle 08 \cdot z$

t1 8 ' $\mathrm{Z}$

$818 \mathrm{Z}$

งะ8' $\mathrm{Z}$

$606{ }^{\circ} \mathrm{Z}$

$926{ }^{\circ} \mathrm{Z}$

$9762^{\circ}$

$\downarrow 乙 6^{\circ} \varepsilon-$
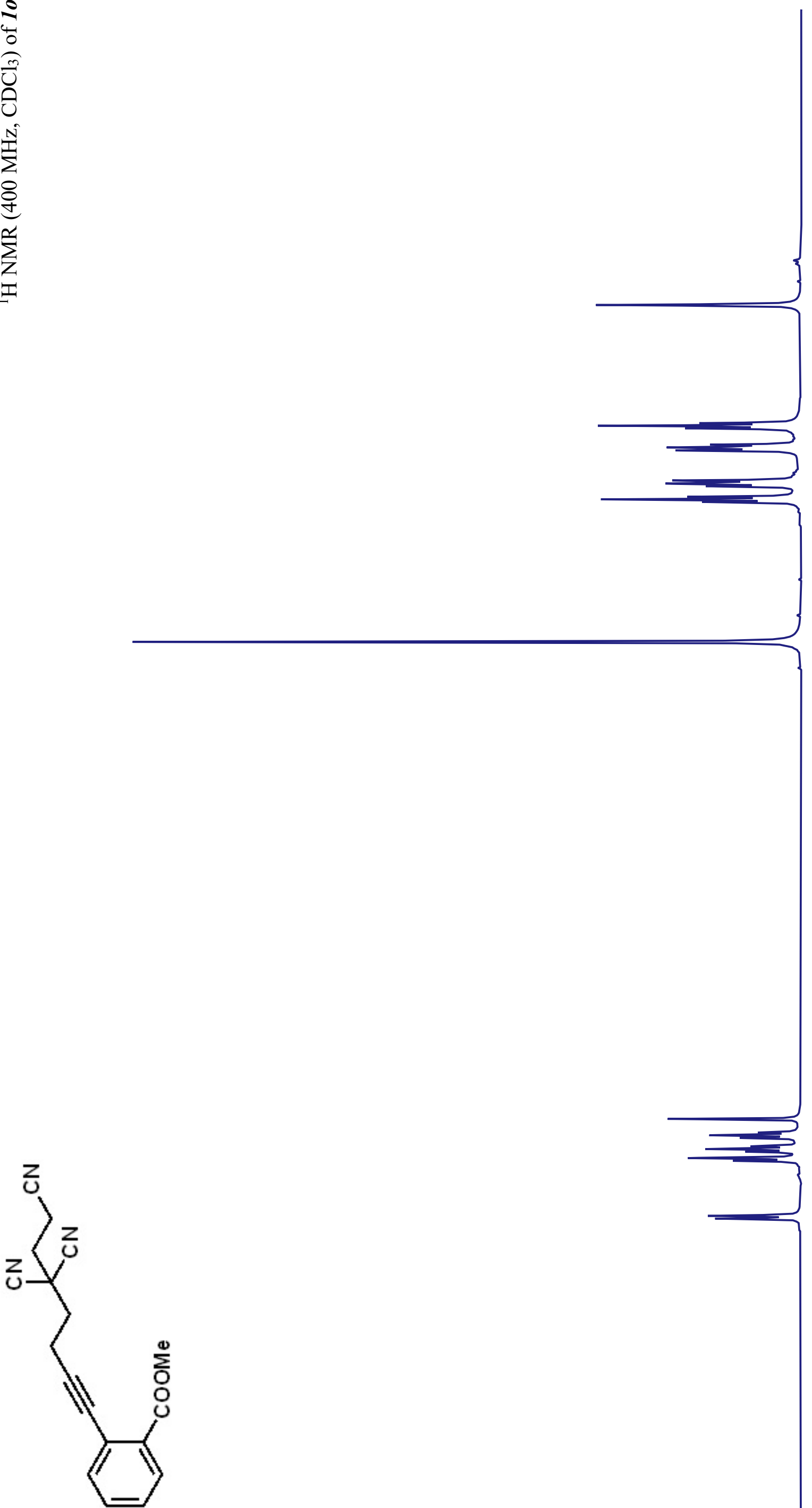

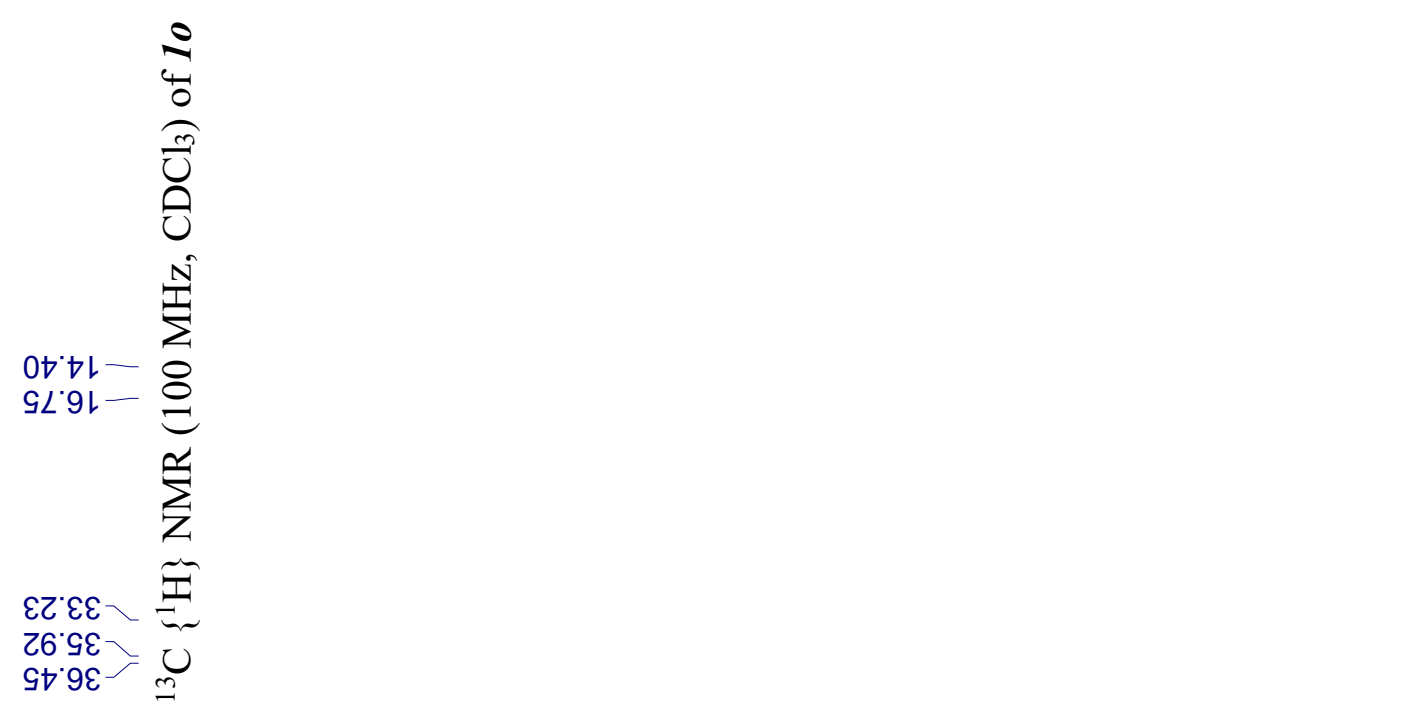

0จฉง-

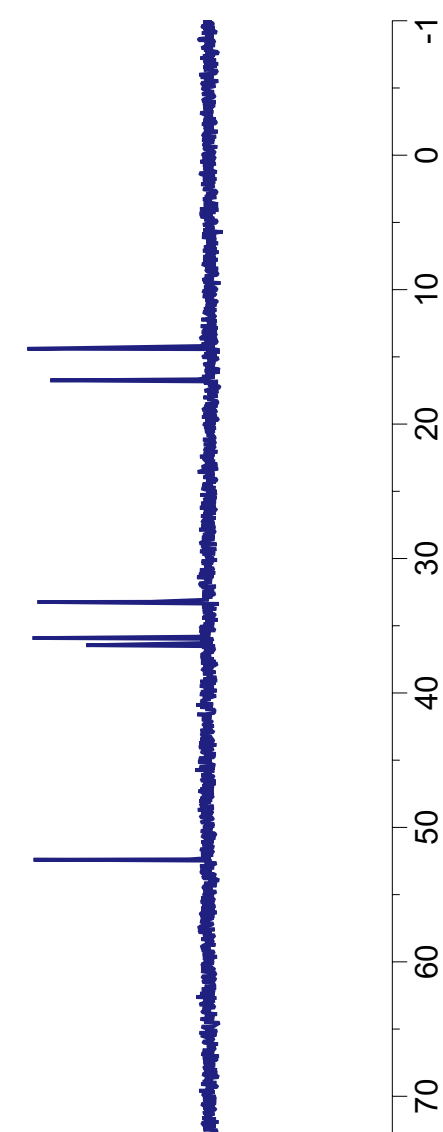

G8. $9 L$

8० $\angle L$

$1 \varepsilon 28$

Sเ:06-

9Lยレー

69.9ルー -

乙๐とてレ

† $8 Z$ L

$\angle G^{\circ} 0 \varepsilon L$

98 เEL

乙0 乙E

ยナ゙
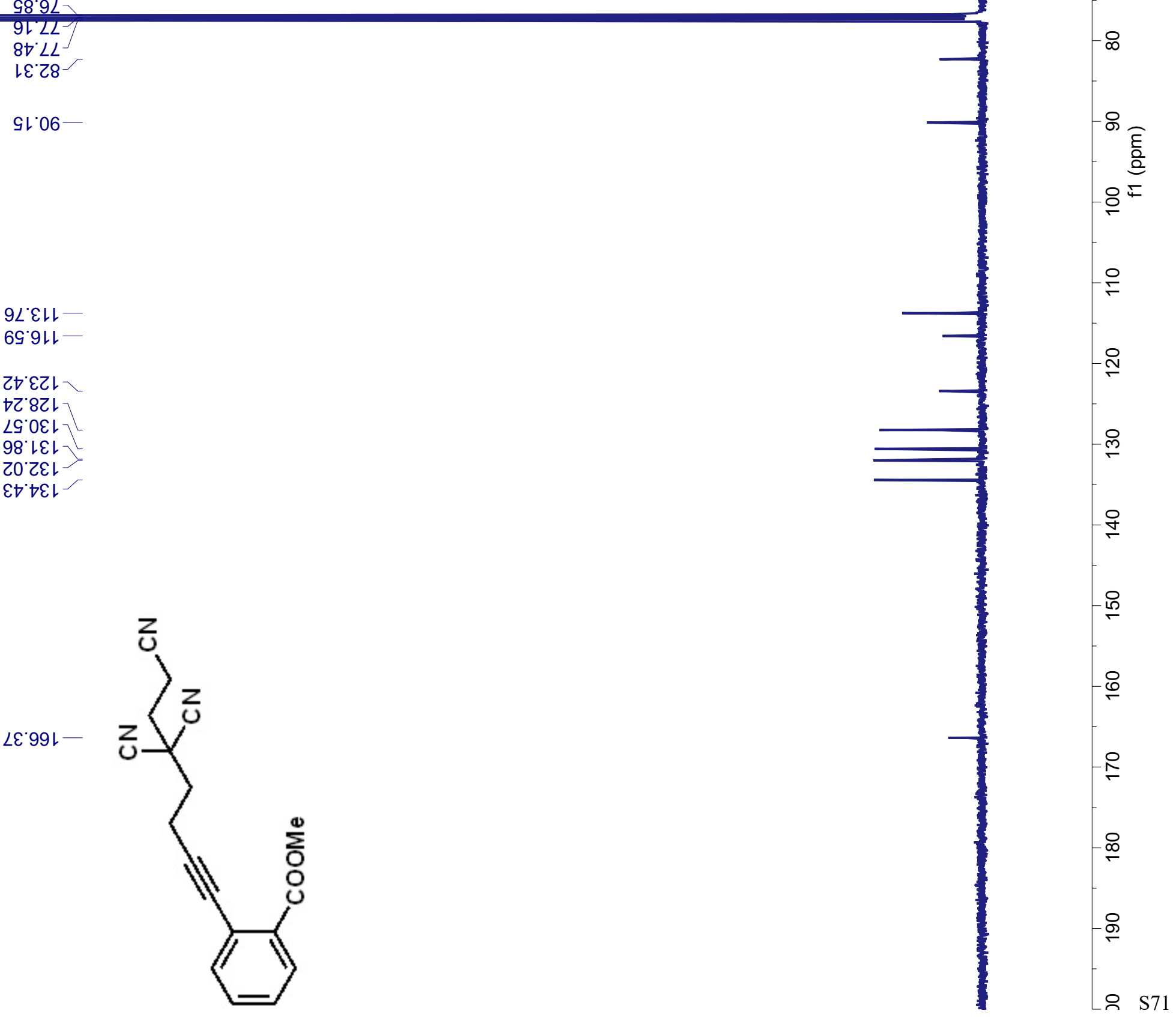


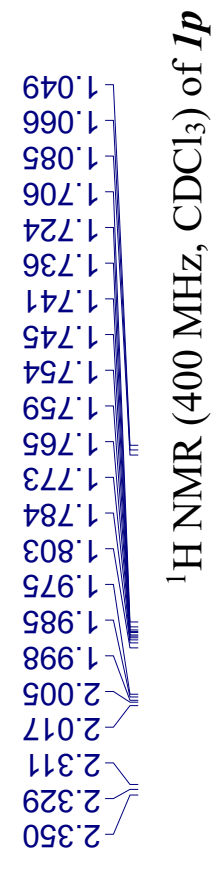

$898^{\circ} \mathrm{Z}$

$888^{\prime} \mathrm{Z}$

$806^{\circ} \mathrm{Z}^{-}$

6เ6 $\varepsilon$

092 L

$6 \varepsilon \varepsilon^{\circ} L$

6SE $L$

$8 \angle \varepsilon^{\circ} L$

$6 \varepsilon t^{\circ} L$

8St $L$

$\angle L D^{\circ} L$

9EG $L-$

SSG. $L$

EL6 $L$

$\varepsilon \varepsilon 6^{\circ} L$

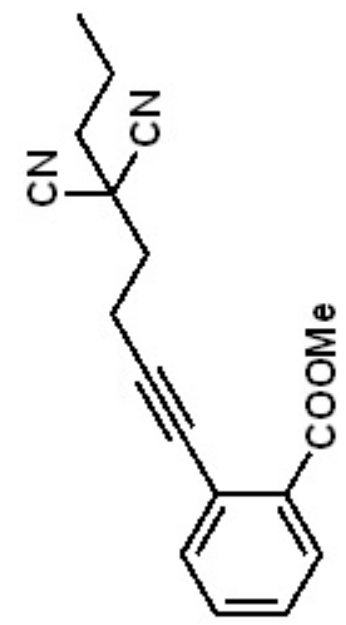

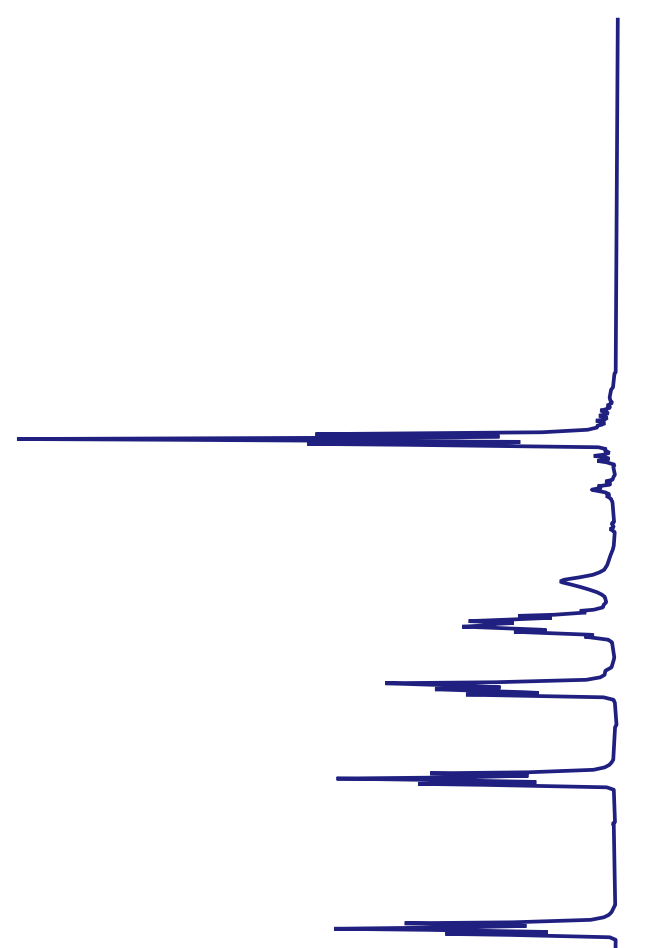

$$
\text { . }
$$

$\left[\begin{array}{c}1 \\ 0 \\ 0 \\ 0\end{array}\right.$

F $96.7 \stackrel{\circ}{\circ}$

F $66^{\circ} \mathrm{L}$

ก

F $86^{\circ} \mathrm{L}$

ले

ก)

$00^{\circ} \varepsilon$

$\stackrel{0}{+}$

ᄂ?

हิ

$0 \leftleftarrows$

(0)

م)

웅

0
0

웅.

$N$

T $06^{\circ} 0$

F96.0

镸 6

N

工 $88^{\circ} 0$

0 

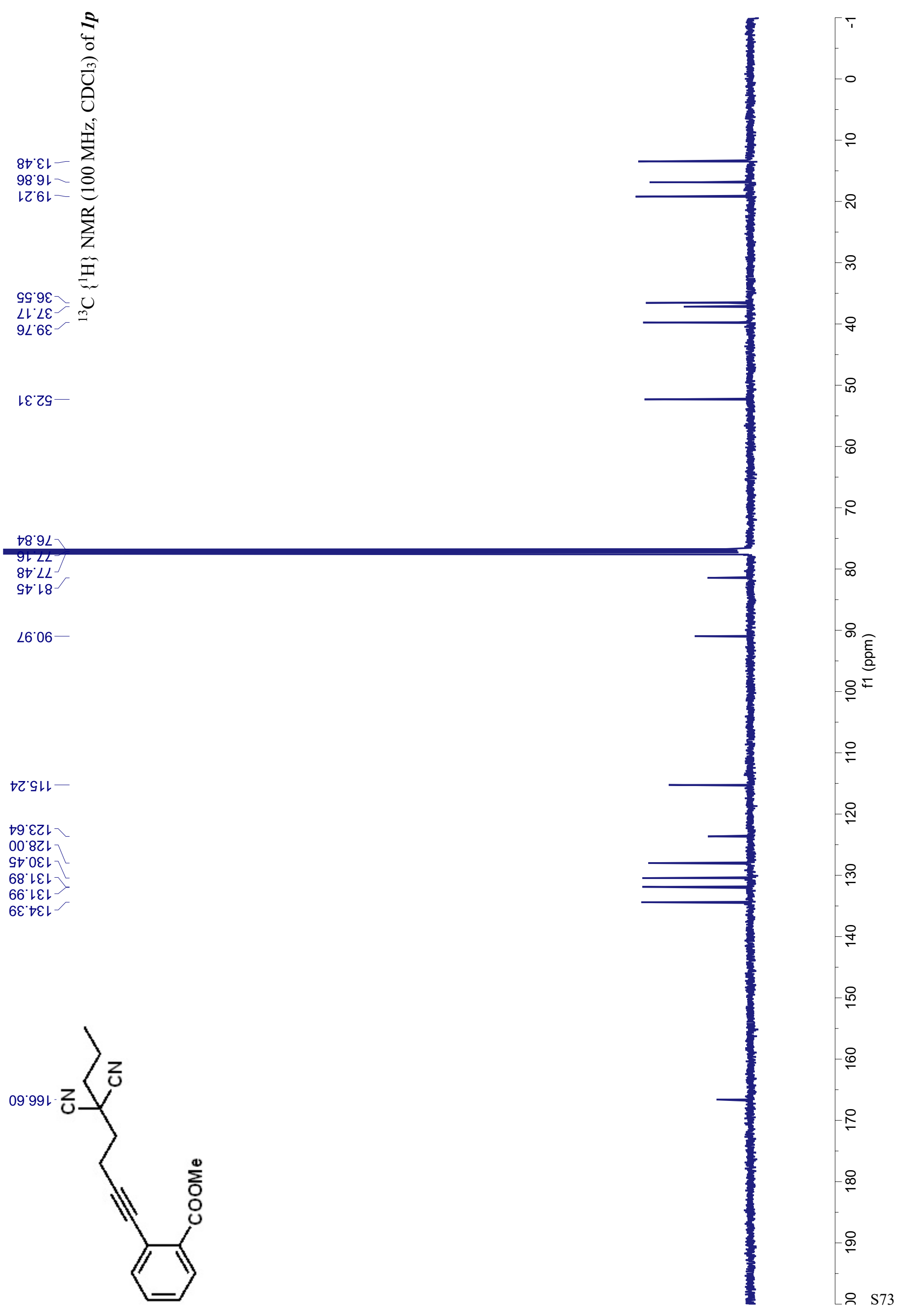


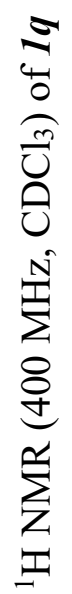

与०ย'乙

$80 \varepsilon^{\prime} Z$

†८ะ

8Z६ 乙

$\downarrow \downarrow \varepsilon 乙$

$8 \nabla \varepsilon^{\prime} 乙$

$\varepsilon L L ' Z$

$Z 6 L^{\circ} Z$

$\nabla \angle 8^{\circ} \mathrm{Z}$

$\angle \angle 8^{\circ} Z$

$968^{\circ} \mathrm{Z}$

Eเ6 $\mathrm{Z}$

$\angle L 6^{\circ} Z$

จเ6 ${ }^{\circ} \varepsilon$

$\left.\neg 8 \varepsilon^{\circ} G\right]$

จレナ

8Lt'

OZt'

Stt 9

9St 9

$69 t 9$

297 9

$0 \angle \nabla^{\circ} 9$

$698^{\circ} 9$

$1 \angle 8^{\circ} \mathrm{G}$

SL8 9

$688^{\circ} 9$

268 9

$\angle 68^{\circ} \mathrm{G}^{\circ}$

$\angle 06^{\circ} 9$

Sเ6. 9

टह6 9

$\angle E 6^{\circ} \mathrm{G}$

096.9

$\angle 96^{\circ} 9$

096 9

SL6'S

6L6 ${ }^{\circ}$

$09 Z^{\circ} \mathrm{L}$

$\checkmark 9 Z^{\circ} \mathrm{L}$

$\neg \varepsilon \varepsilon^{\circ} L$

$8 \varepsilon \varepsilon^{\circ} L$

ESE $L$

$\angle G \mathcal{E}^{\circ} \angle$

$9 \angle E^{\circ} L$

$9 \angle E^{\circ} L$

EEt $\angle$

$\angle E \nabla^{\circ} L$

ZSt $L$

99t $L$

$\nabla \angle t^{\circ} \angle$

$G \angle D^{\circ} \angle$

8ES $L$

$\angle S S^{\circ} L$

$606^{\circ} \mathrm{L}$

$626^{\circ} \mathrm{L}$
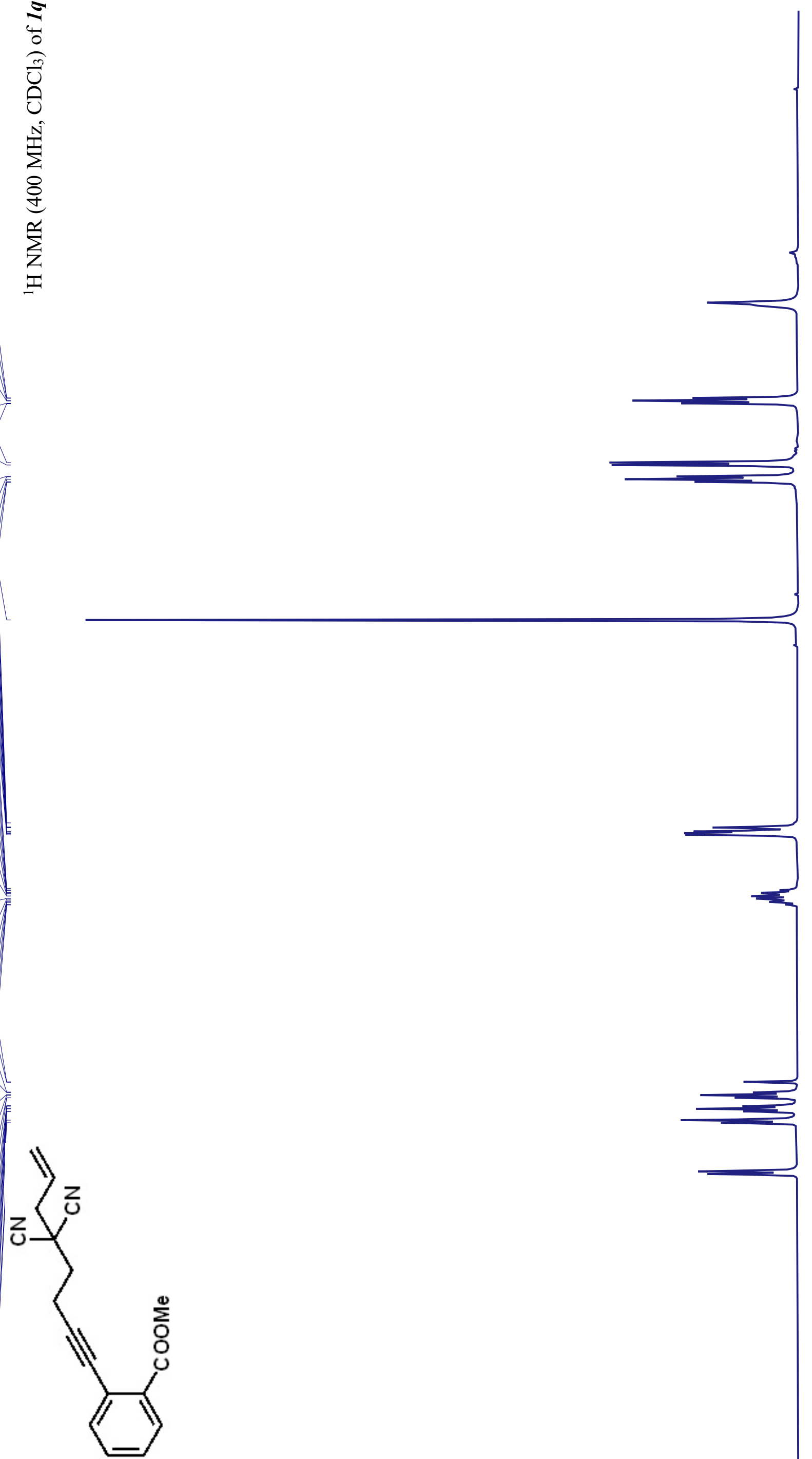


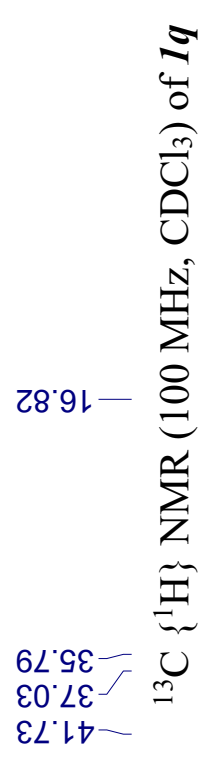

०ะ २९-

$78 \cdot 9 L$

$9 L^{\circ} L L$

8t $\angle L$

$\mathrm{S}^{\circ} 18$

$18.06-$

8ட゙จレー

$\angle G^{\circ} \varepsilon Z L$

L $' E Z L$

Z० 8Z

ह† 8ZL

$\varepsilon \nabla^{\circ} 0 \varepsilon L-$

88. LL -

เ० ZEL

$8 \varepsilon^{\circ} \downarrow \varepsilon\llcorner$
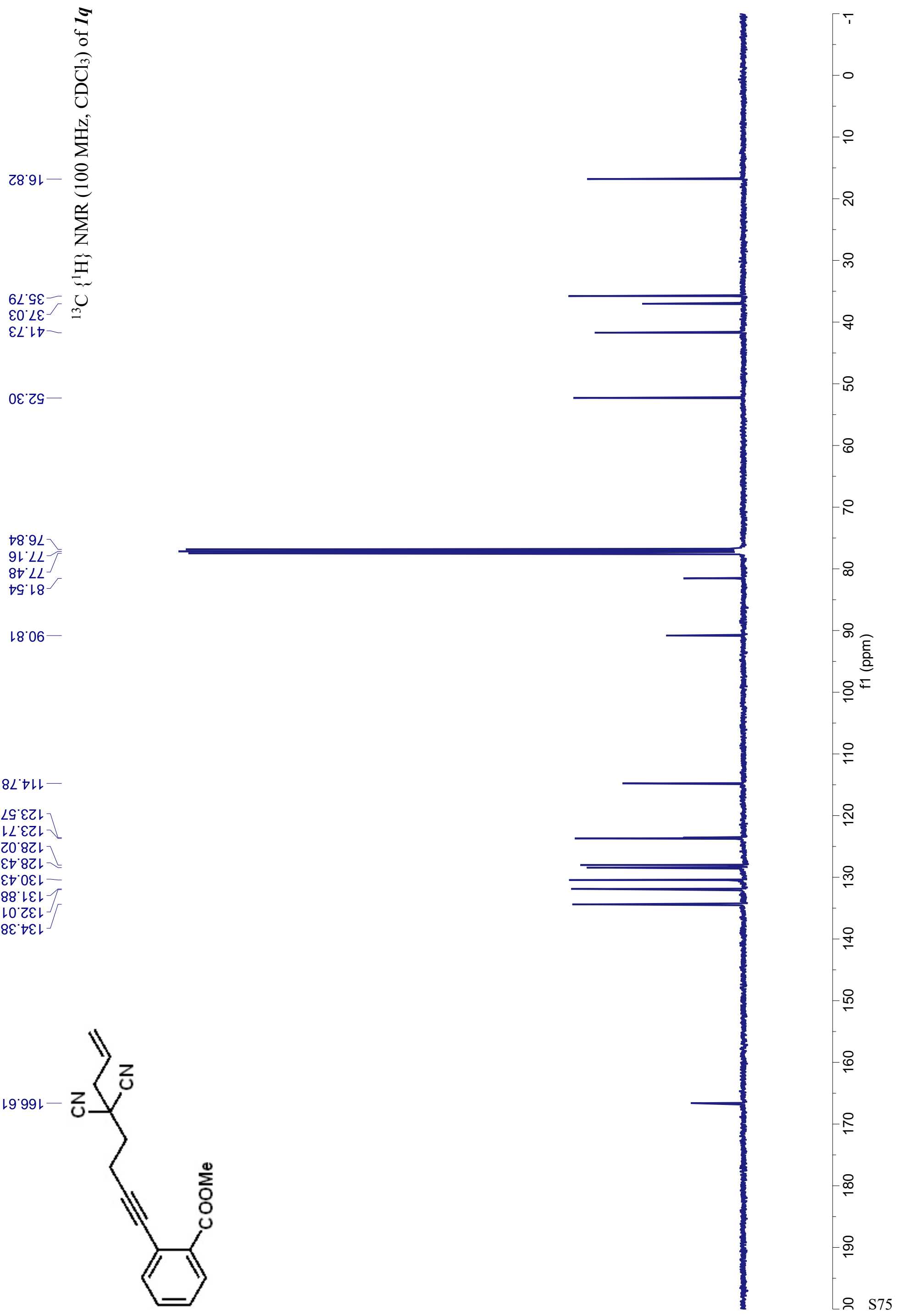


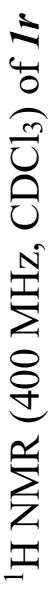

$\angle E L L-$

928 '

乙6乙 乙

ㄴㄷ

†เE'

乙ย६'

$8 \mathrm{D} \cdot \mathrm{Z}$

$\angle 9 L Z$

Z98 $\mathrm{Z}$

Z88'

$106^{\circ}{ }^{\circ}$

งเ6 $6^{\circ}-$

$682^{\circ} 9$

$80 \varepsilon^{\circ} \mathrm{G}$

$8 Z \varepsilon^{\circ} \mathrm{G}$

$09 Z^{\circ} L$

SEE $L$

$\neg G \mathcal{E}^{\circ} \mathrm{L}$

$69 \varepsilon^{\circ} \angle$

$\varepsilon \angle \varepsilon^{\circ} L$

00t $L$

$\nabla \varepsilon \nabla^{\circ} L$

$\nabla S \nabla^{\circ} L$

$\varepsilon \angle \nabla^{\circ} L$

6ES $L$

89. $L$

$\angle 06^{\circ} \mathrm{L}$

$\angle Z 6^{\circ} \mathrm{L}$
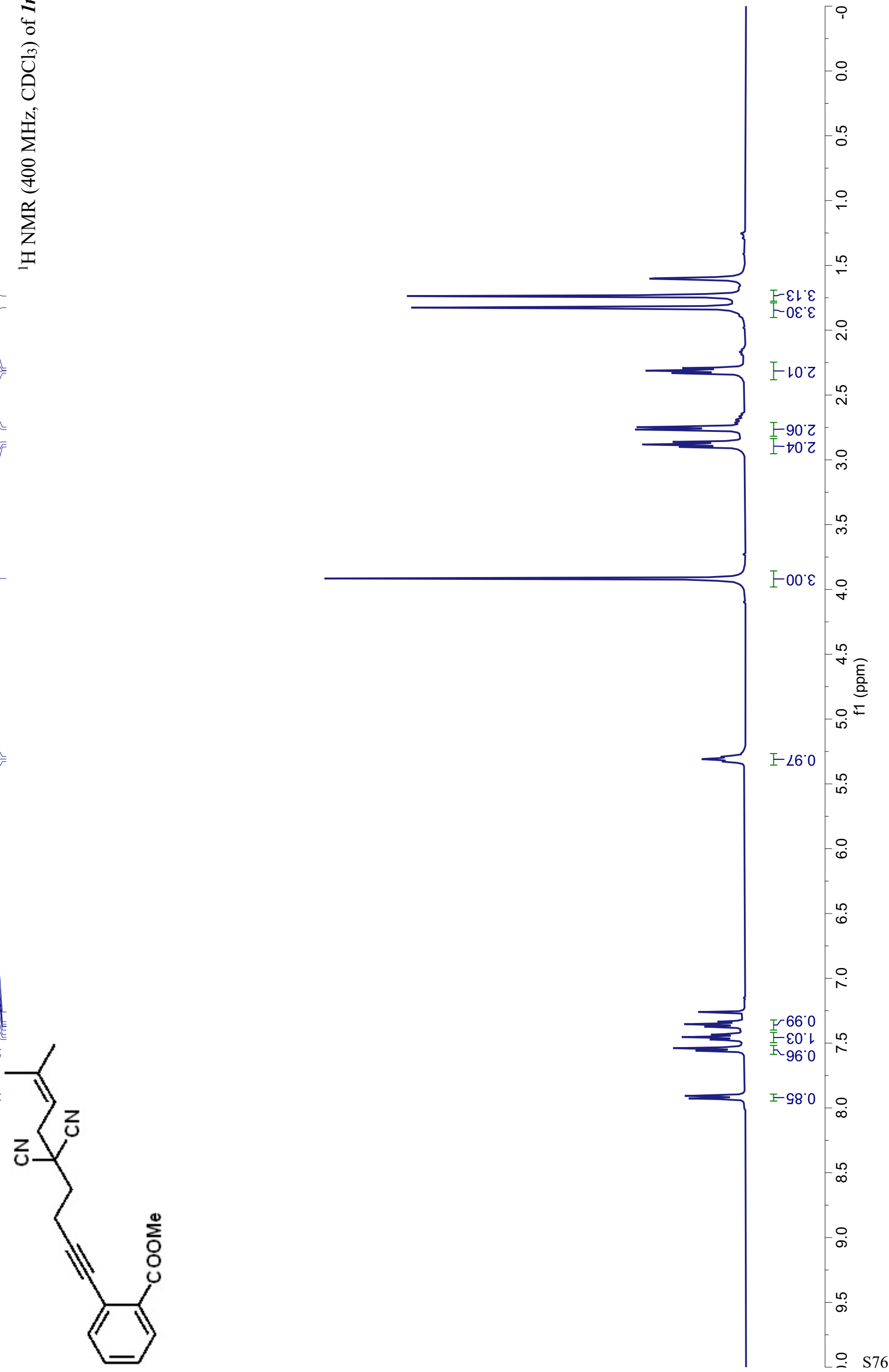


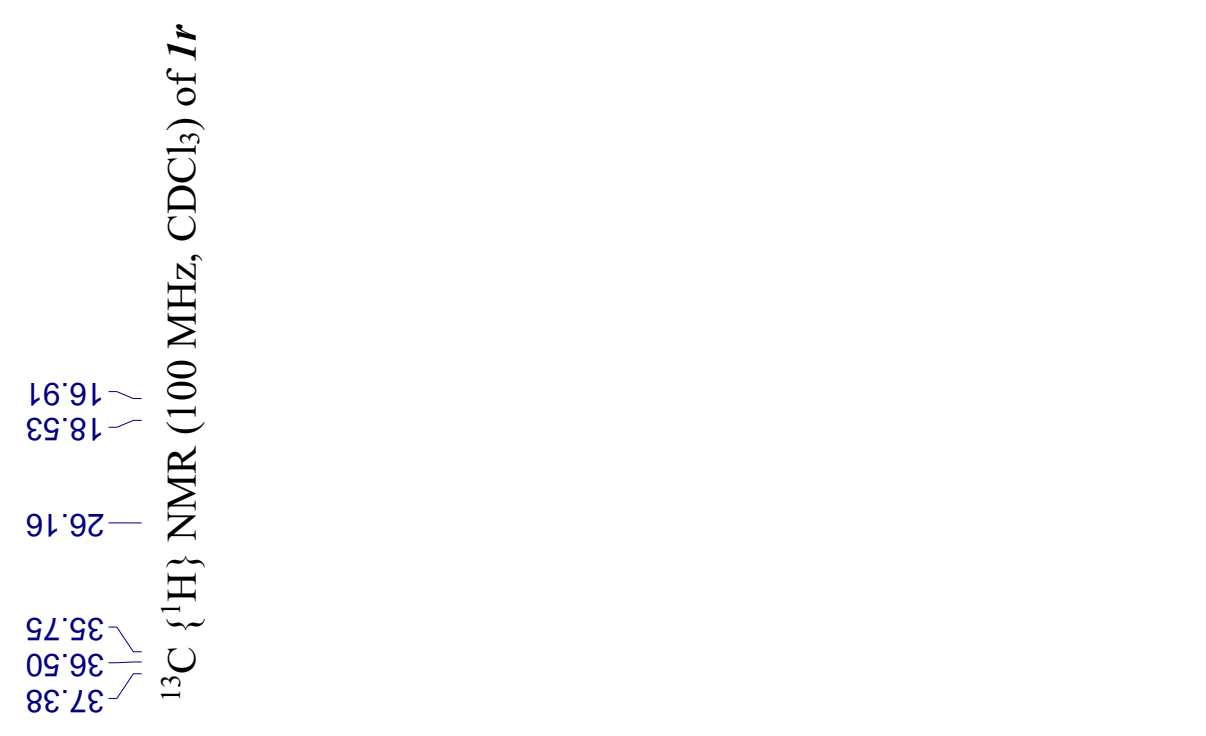

8२'२9-

78.92

$9 L^{\circ} L L$

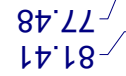

t0. 6

8ヤ゙เレ

†乙งル

† ${ }^{\circ} \varepsilon 乙 レ$

$86^{\circ} \mathrm{LCL}$

E७ OEL

L8'LEL

ᄂ० ZEL

$8 \varepsilon^{\circ} \downarrow \varepsilon\llcorner$

0てレャー
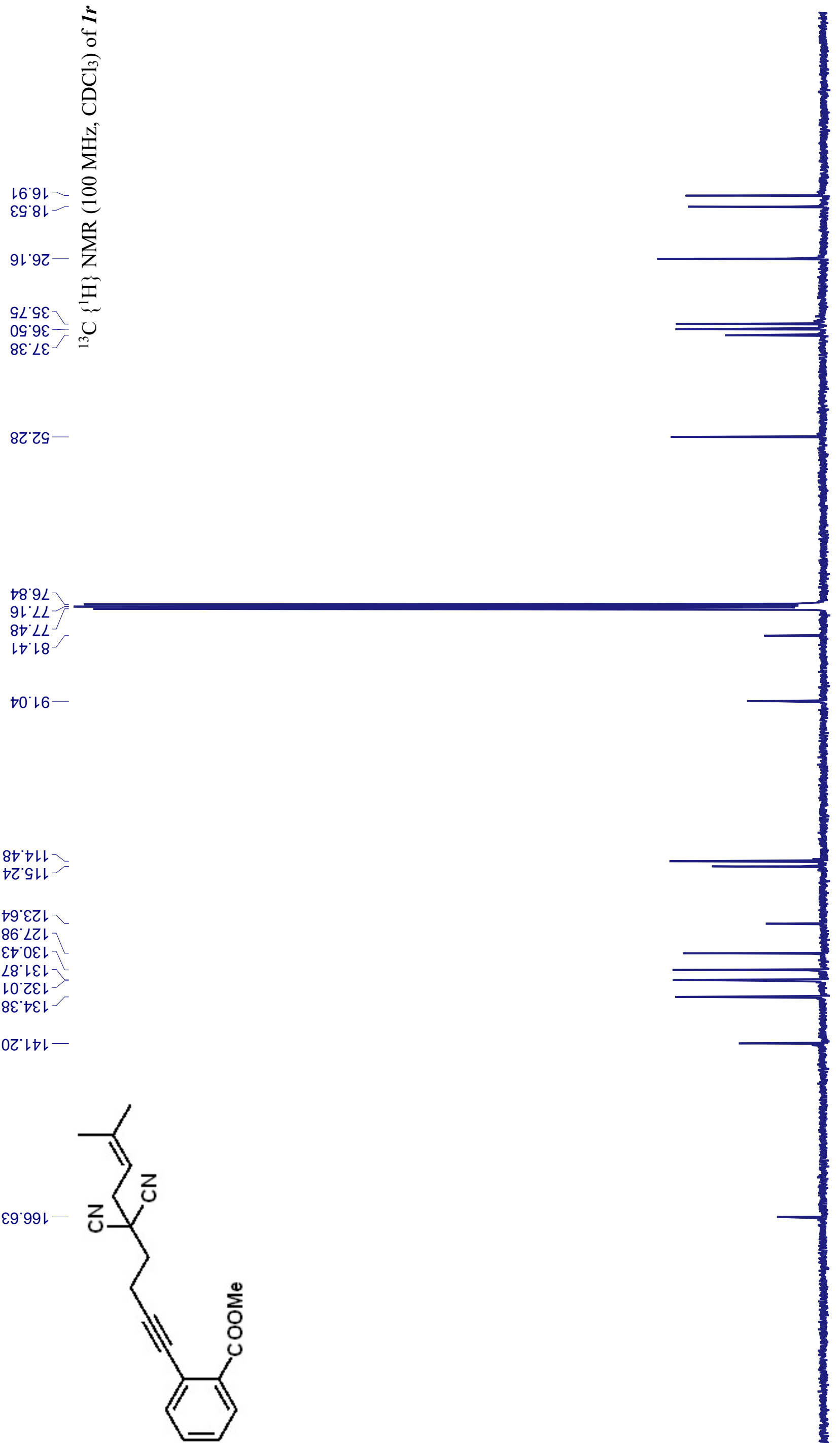


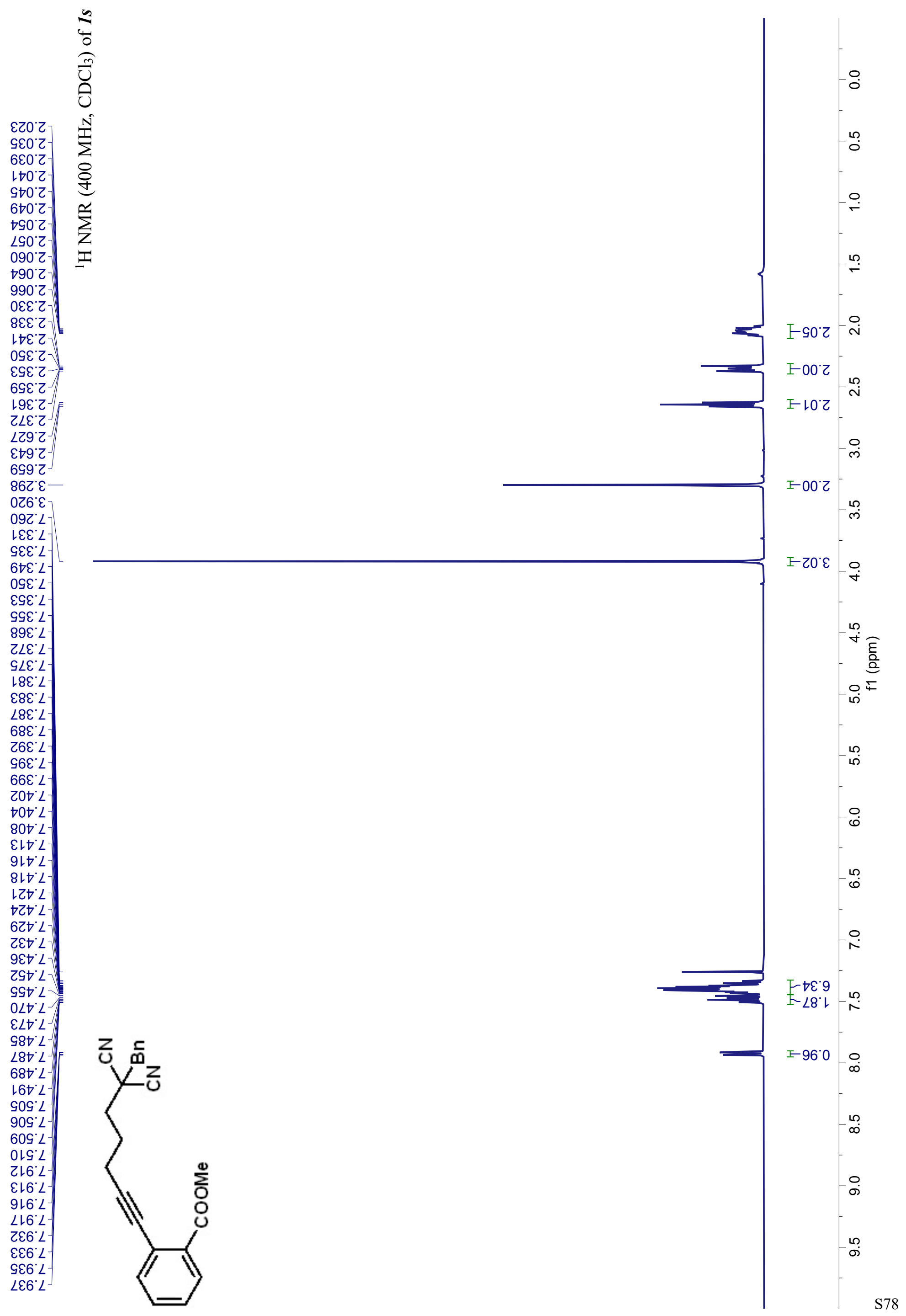



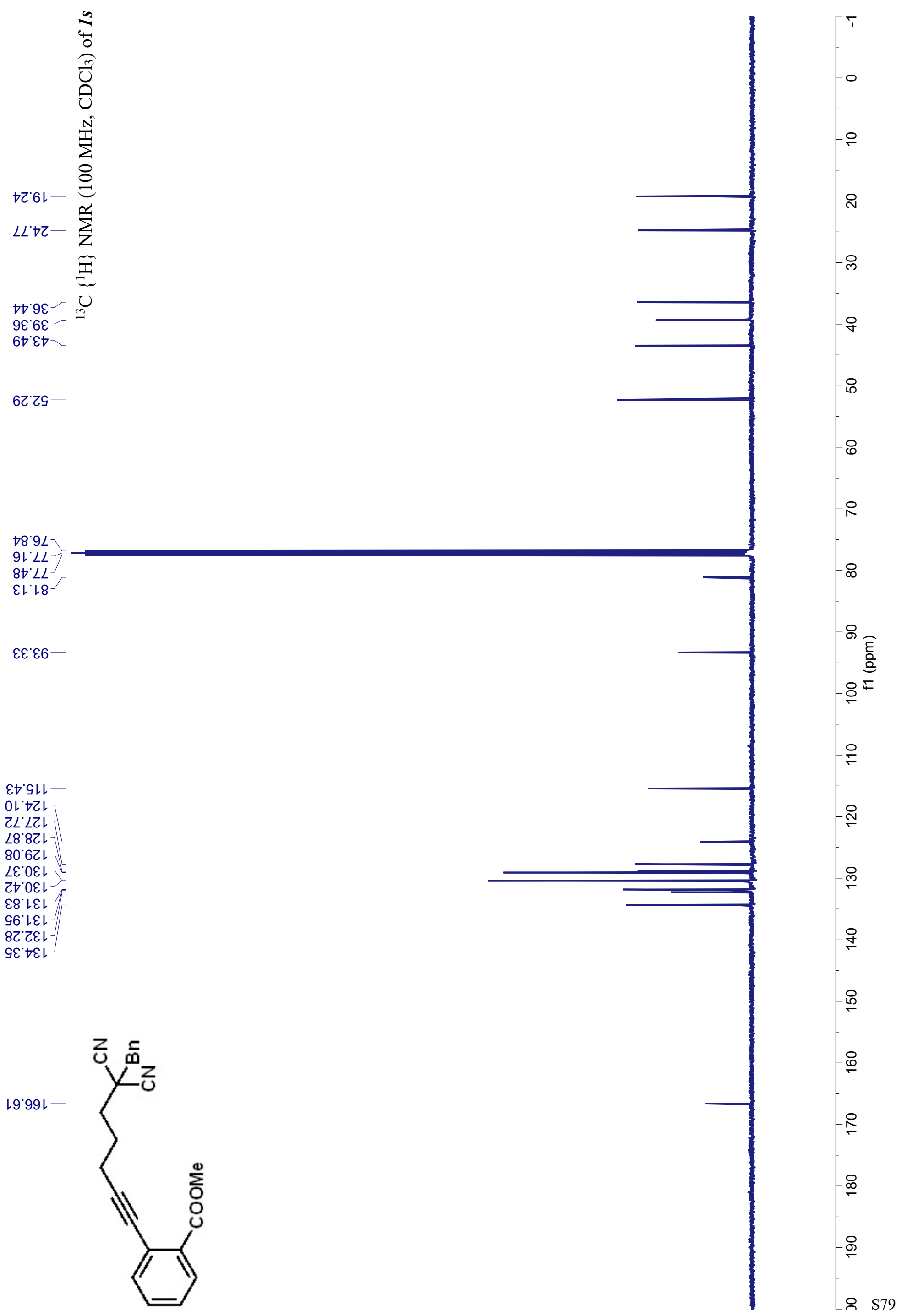


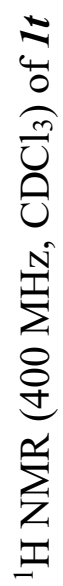

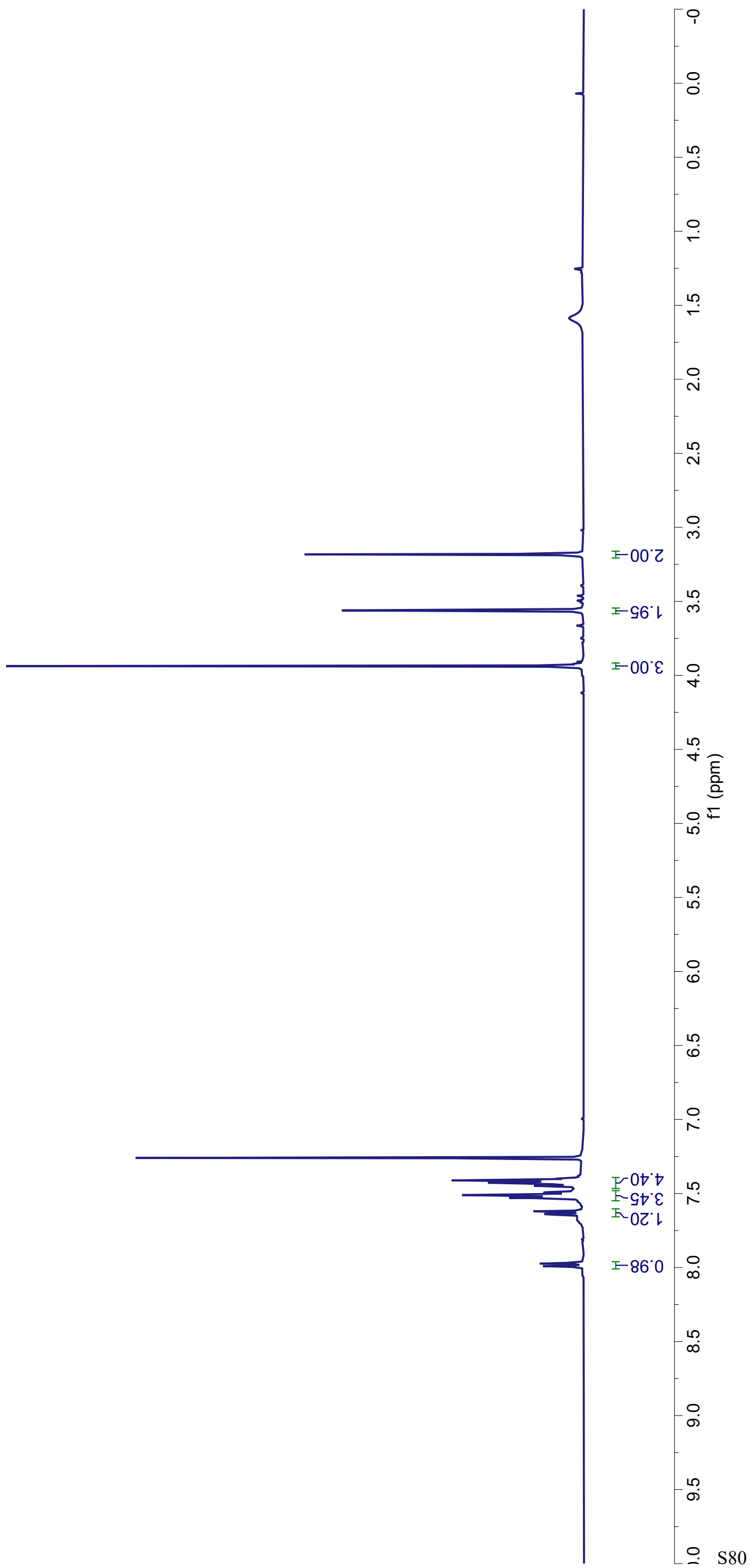



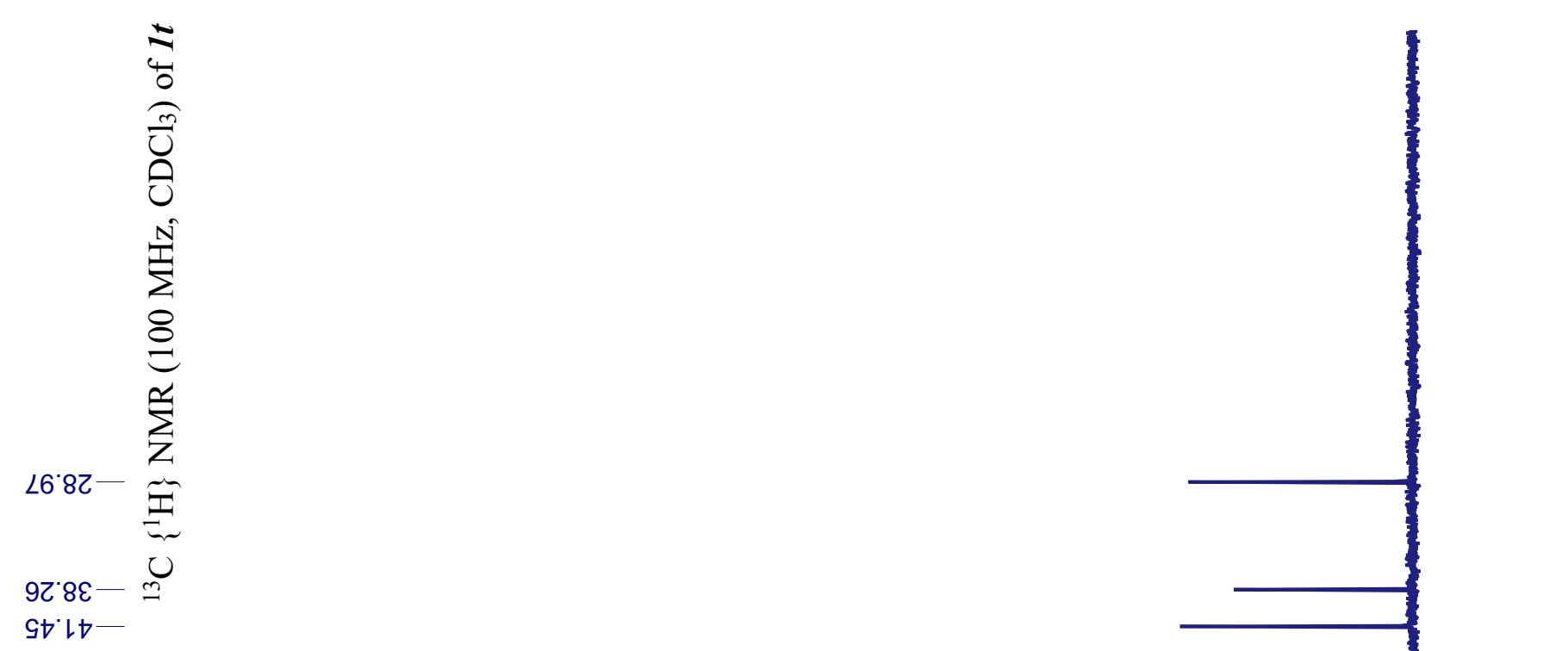

Z9'29-

$78 \cdot 9 L$

$9 L^{\circ} L L$

$26{ }^{\circ} \mathrm{\circ} 8$

00.98

GLナルレ -

99'ててレ-

18.8Z

$00^{\circ} 62$

9l.6ZL

$\mathcal{S}^{\circ} \mathrm{OEL}$

$09^{\circ} 0 \varepsilon L$

乙6'Lレ

เ० ZEL

¿乙'乙E

$08^{\circ} \downarrow \varepsilon L$

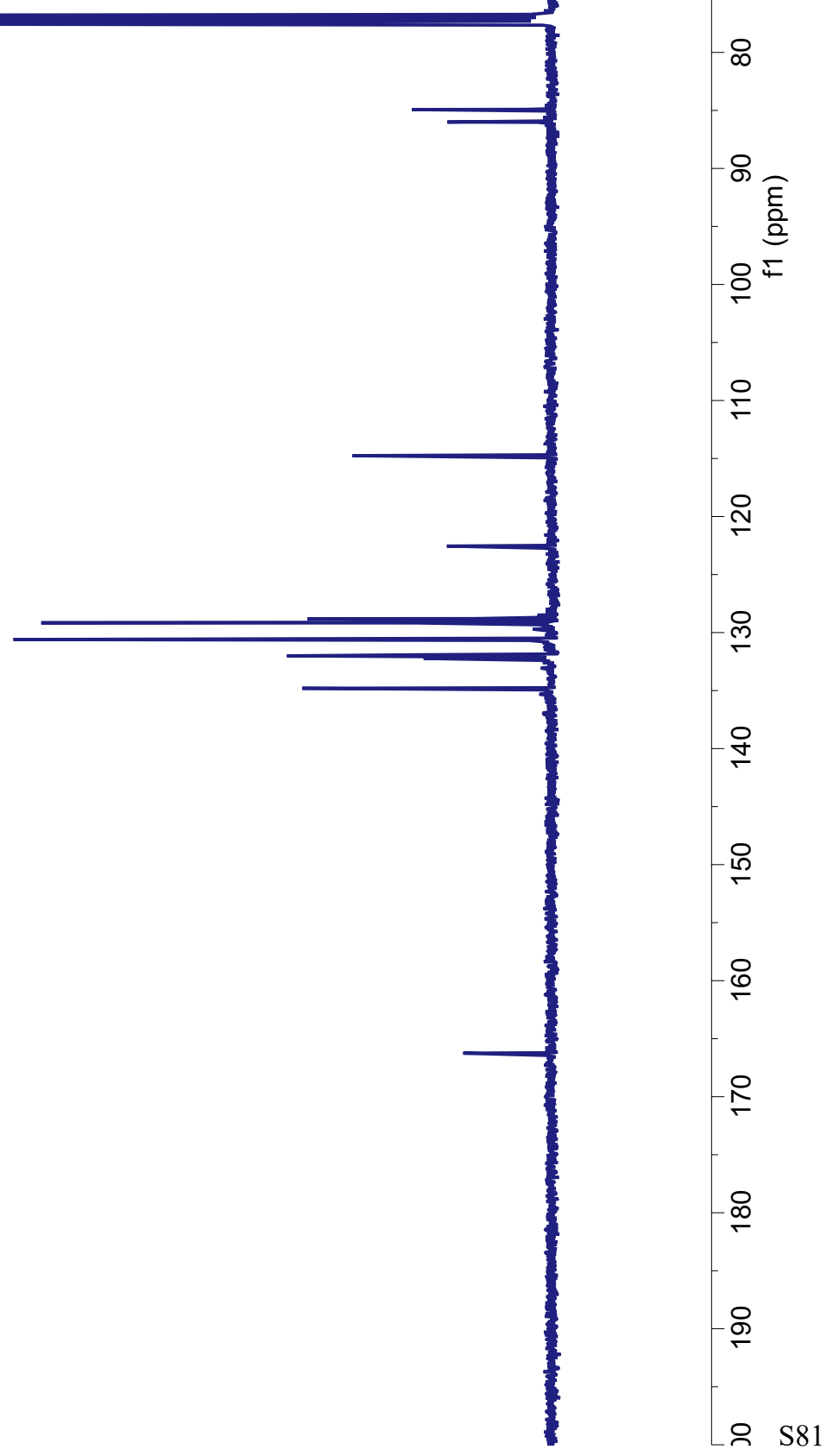




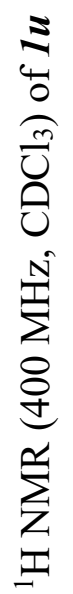

6ระ 乙

$6 \angle \varepsilon^{\prime} 乙$

00t 2

ESt乙

$\angle \nabla 6^{\circ} Z$

$\angle 966^{\circ} \mathrm{Z}$

$\angle 86^{\circ} \mathrm{C}^{-}$

๑๐ $\mathcal{\varepsilon}^{\circ} \varepsilon-$

$88^{\circ} \varepsilon-$

IZZ L

$1 \nabla Z \angle$

$09 Z^{\circ} L$

$19 \varepsilon^{\circ} L$

$08 \varepsilon^{\circ} L$

$807^{\circ} \mathrm{L}$

$869^{\circ} \mathrm{L}$

$8 \mathrm{~L} L \mathrm{~L}$
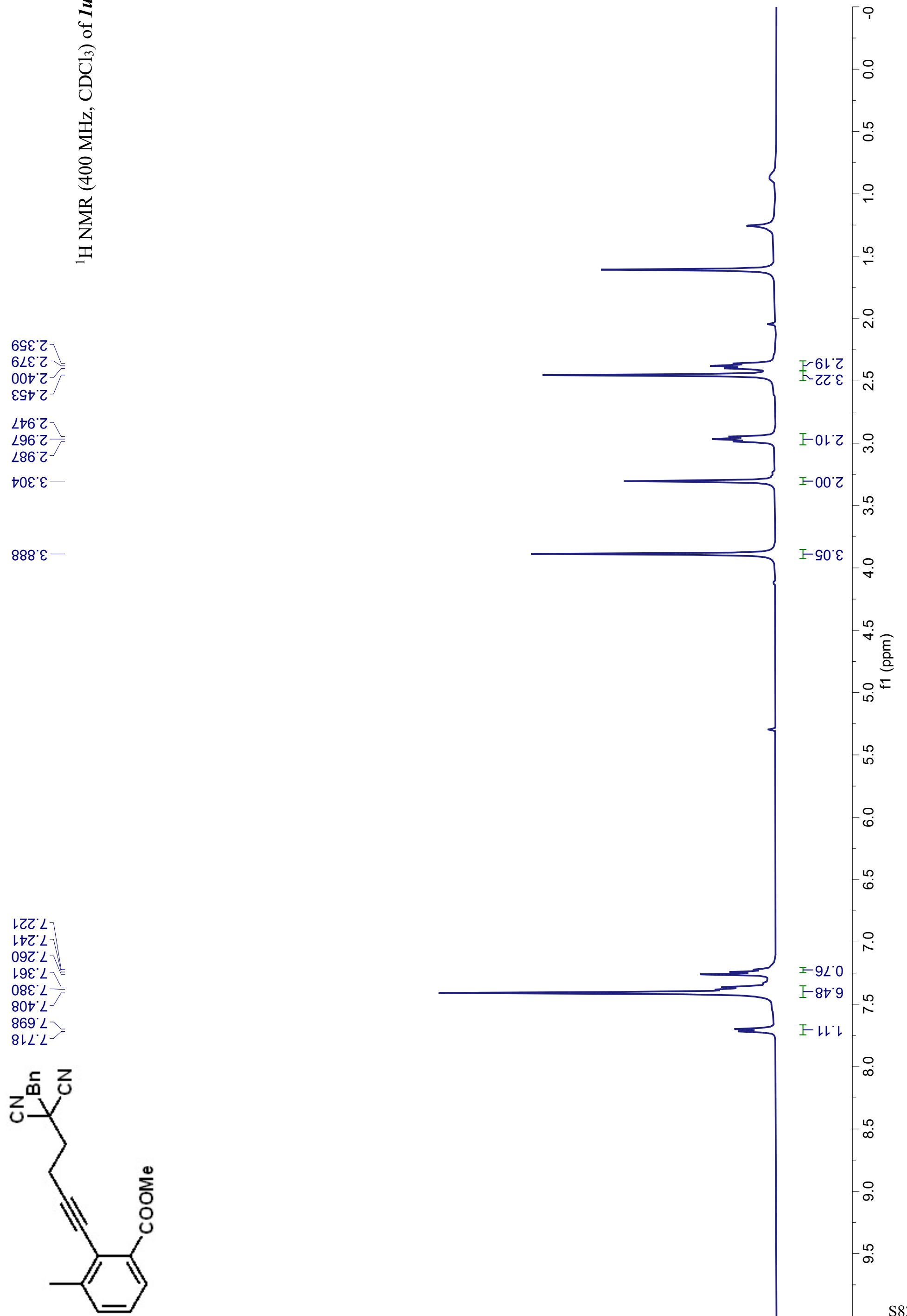


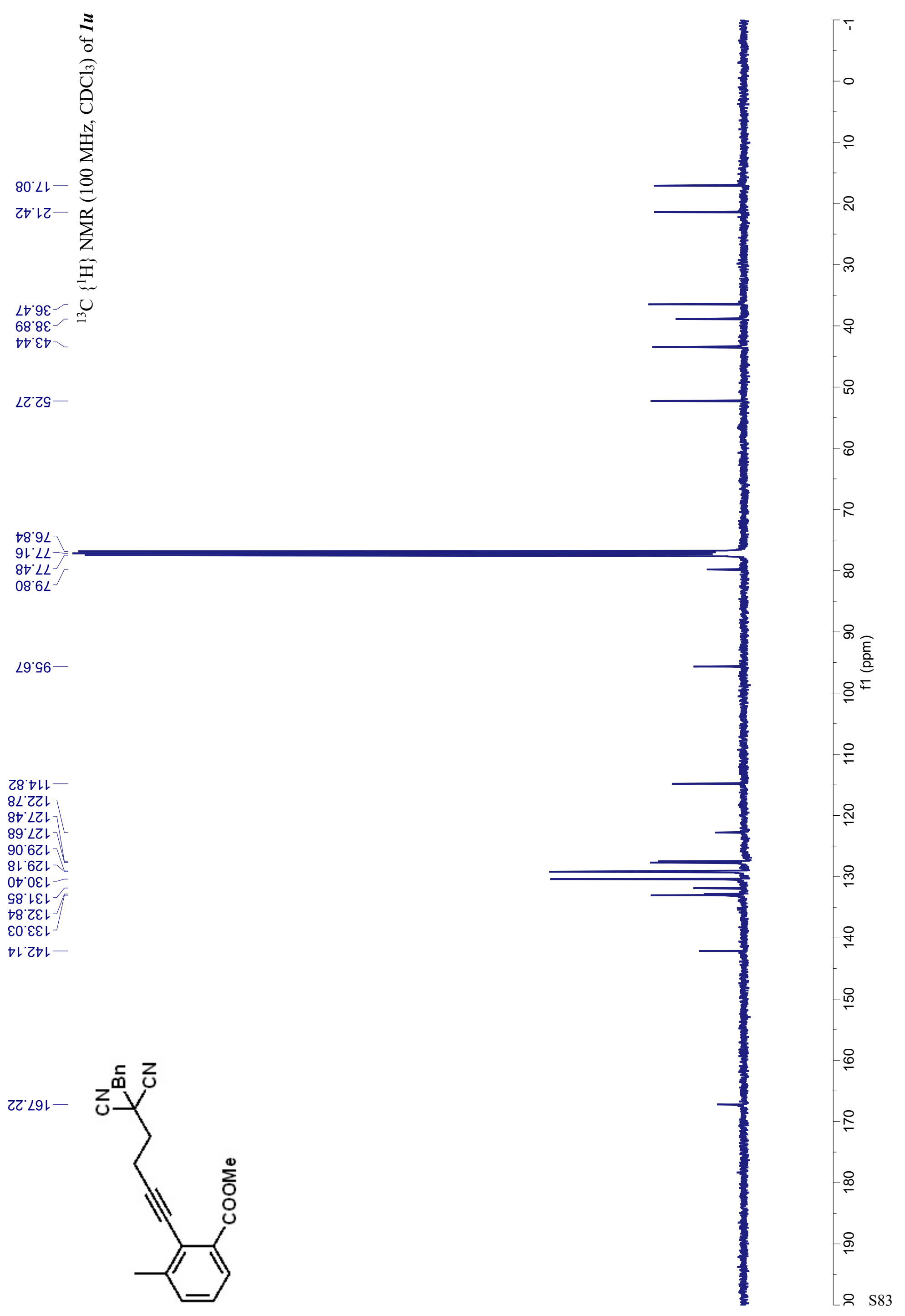




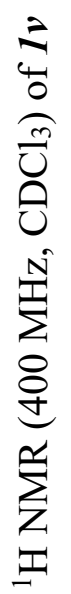

0927

$0 \angle Z ' Z$

68乙 乙

$乙 \varepsilon^{\prime} 乙$

6เ8 2

6ย8 乙-

$698^{\circ} 乙^{-}$

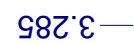

$206^{\circ} \varepsilon-$

OSL $L$

$69 \mathrm{~L} L$

$6 \mathrm{LC} L$

$8 \varepsilon Z^{\circ} L$

$092 \mathrm{~L}$

$88 Z^{\circ} \mathrm{L}$

$\angle O E^{\circ} \angle$

$80 t^{\circ} \mathrm{L}$
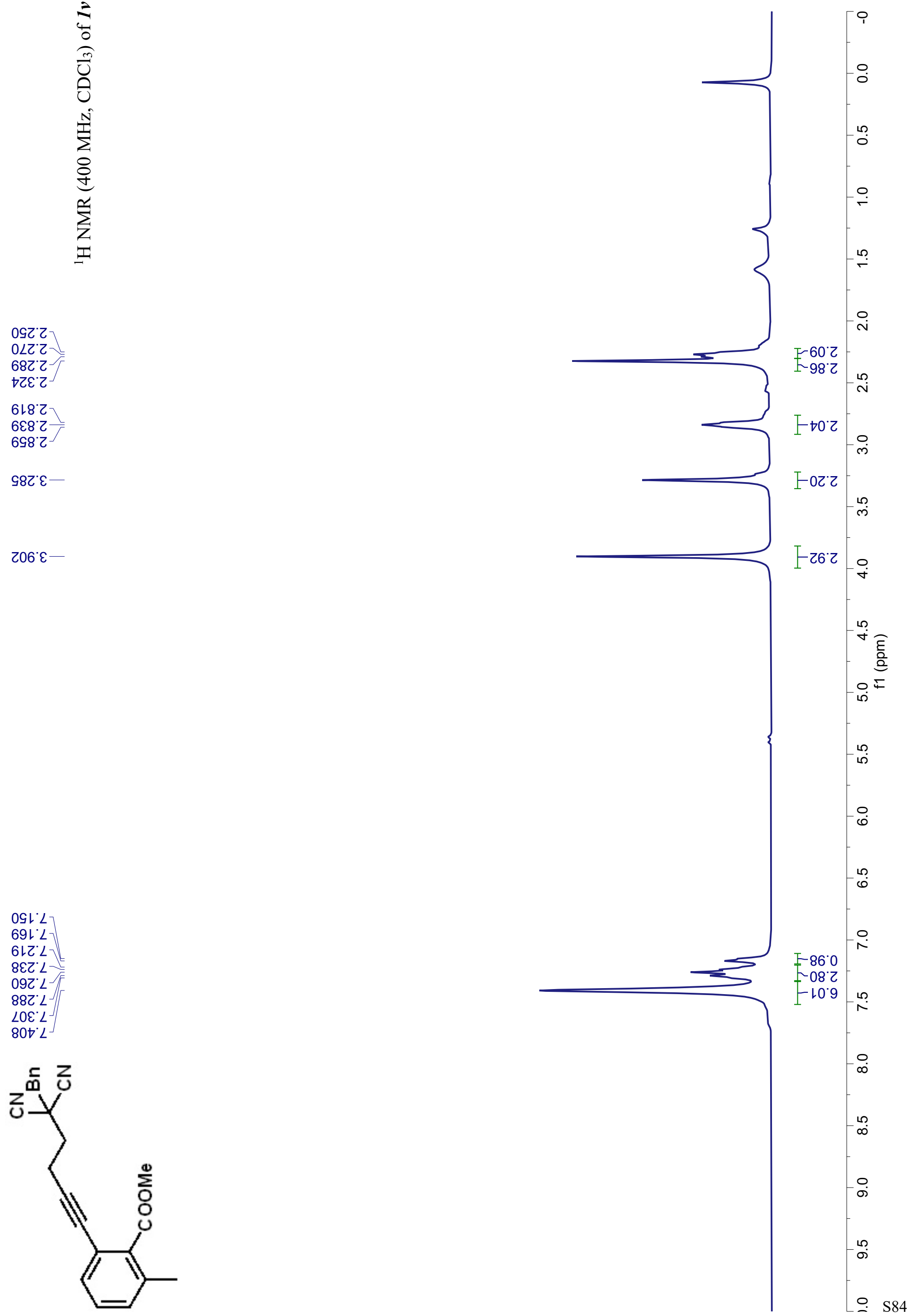


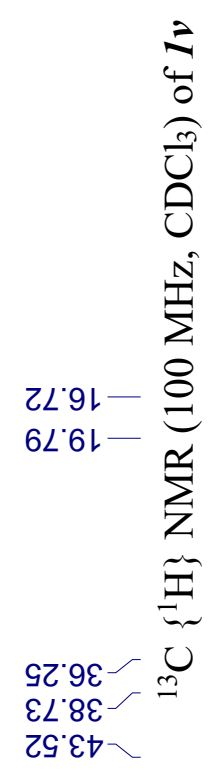

E

$\varepsilon L \cdot 8 \varepsilon$

$8 \mathcal{8}^{\circ} \mathrm{CS}$

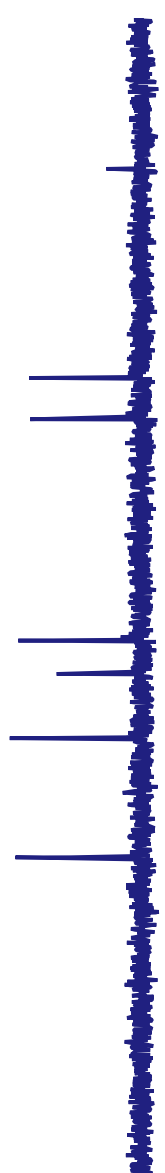

$78^{\circ} 9 L$

$87^{\circ} L L$

$89^{\circ} 08^{\circ}$

†8'88-

U6ZL

乙Z 6ZL

87 6ZL

LO $0 \varepsilon L$

$\angle \varepsilon^{\circ} 0 \varepsilon L$

レ๐०とレ

$\varepsilon L I E L$

GG'GEL
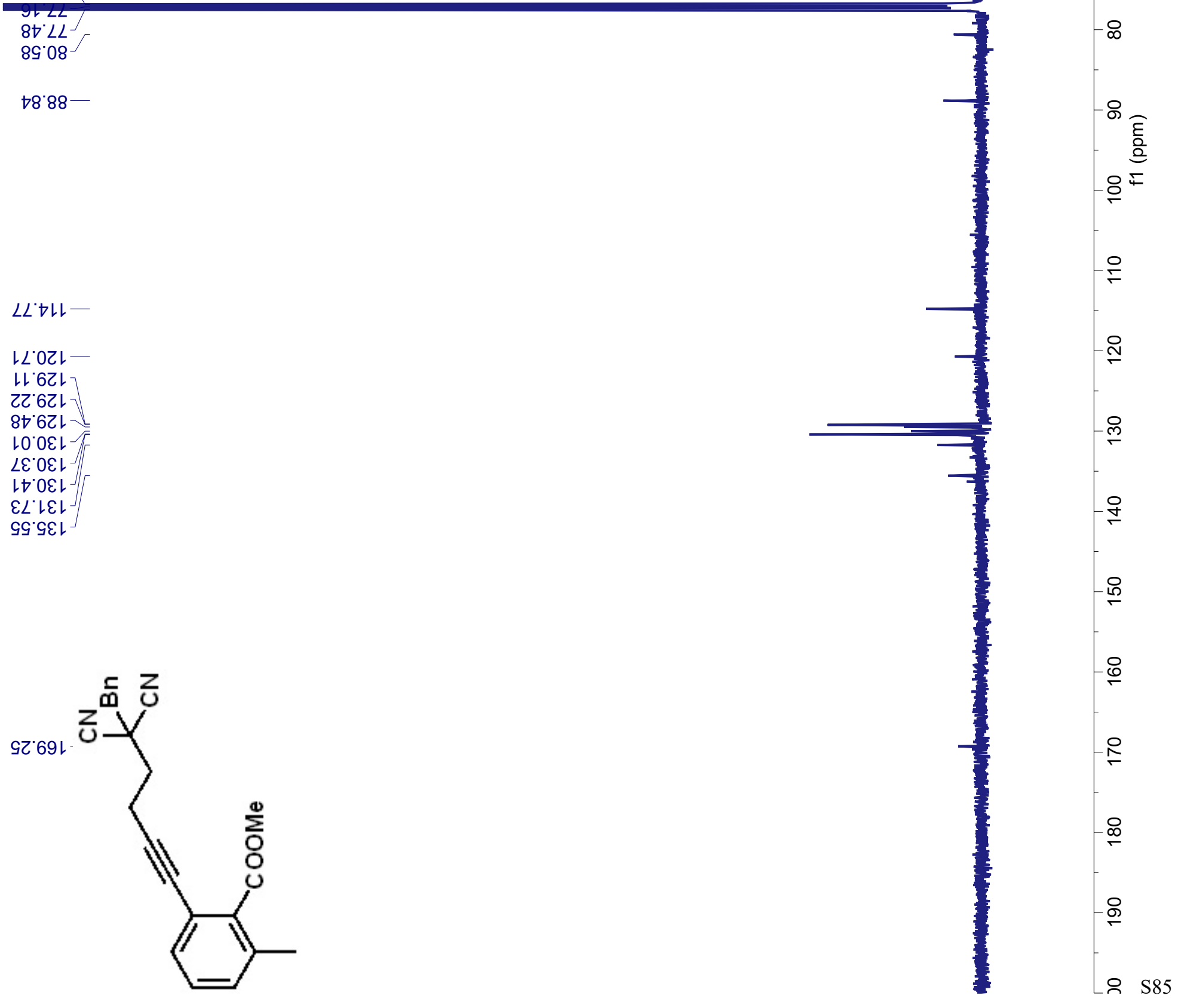

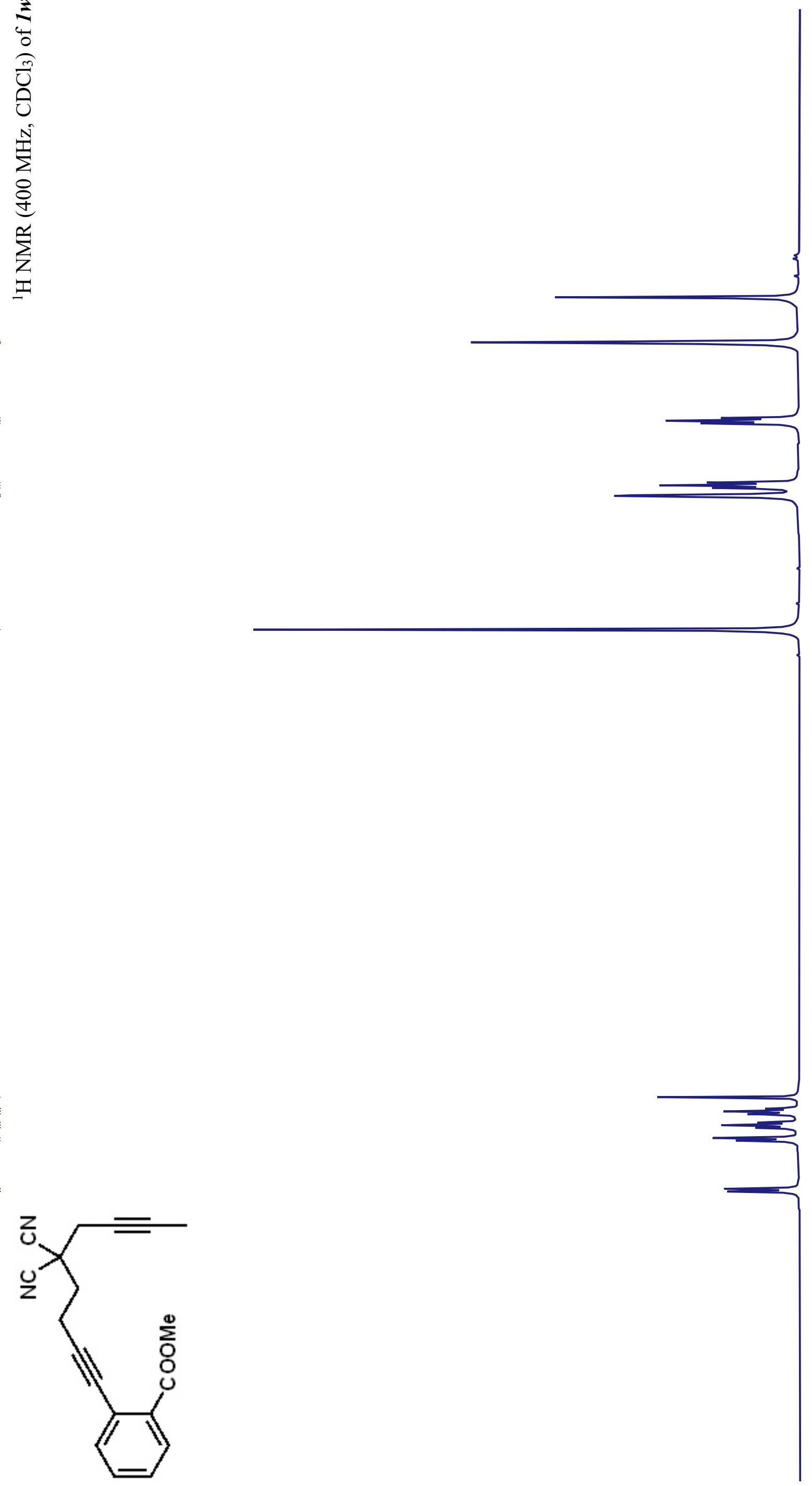

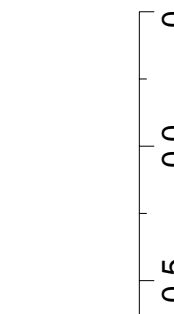
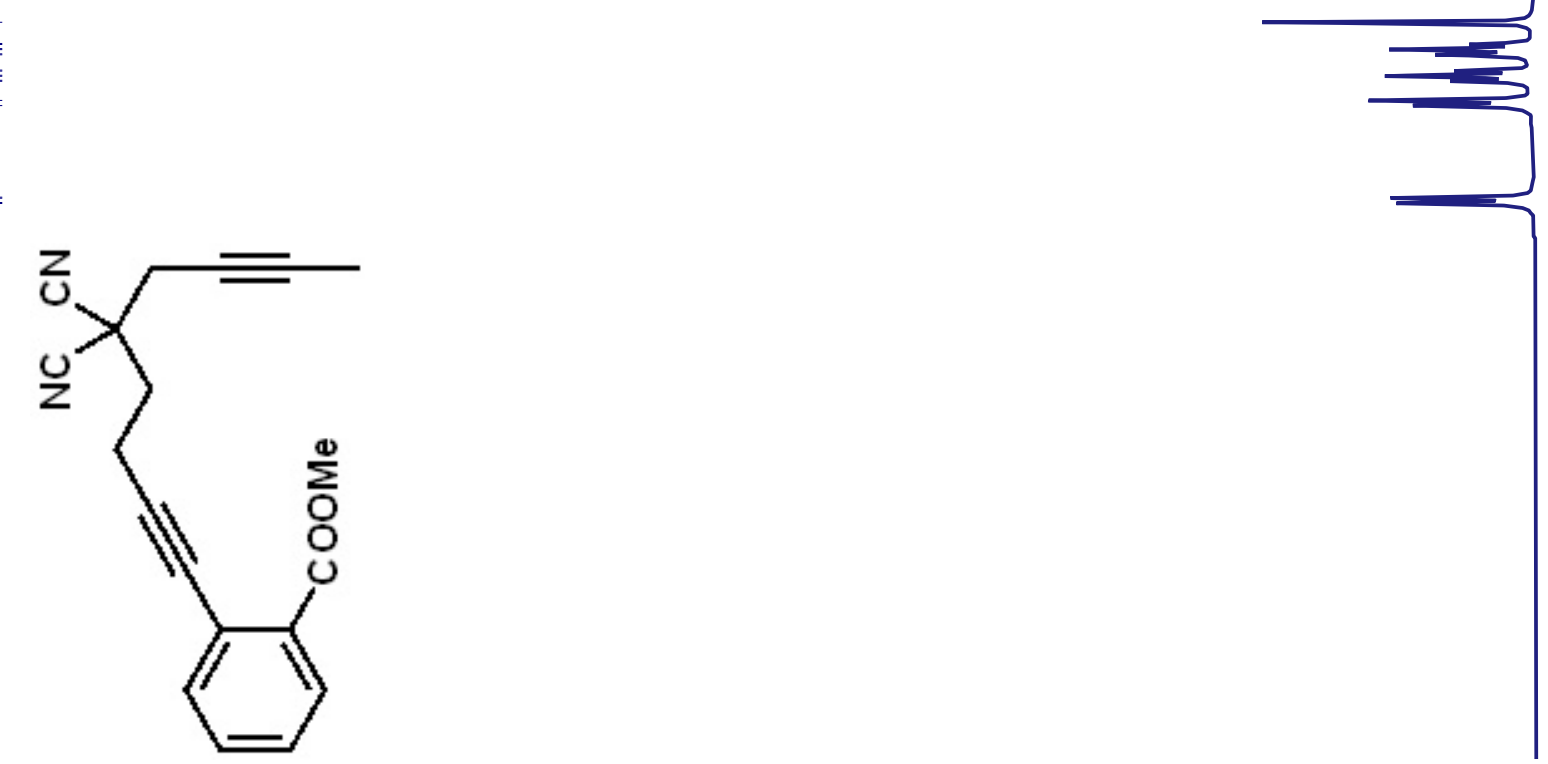


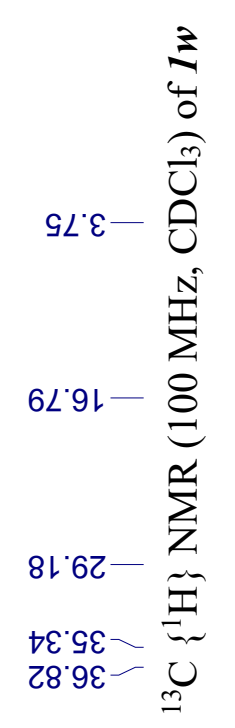

$\left\llcorner\mathcal{E}^{\prime} Z \mathrm{G}-\right.$

09.69-

†8.9L

8t $2 L$

E9. 18

$0{ }^{\circ} \varepsilon 8$

乙L'06-

89`ャレー

89. ๕てレ

$70.8 \mathrm{~L}$

St.0ع

$06 . L E L$

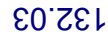

レナ゙とレ

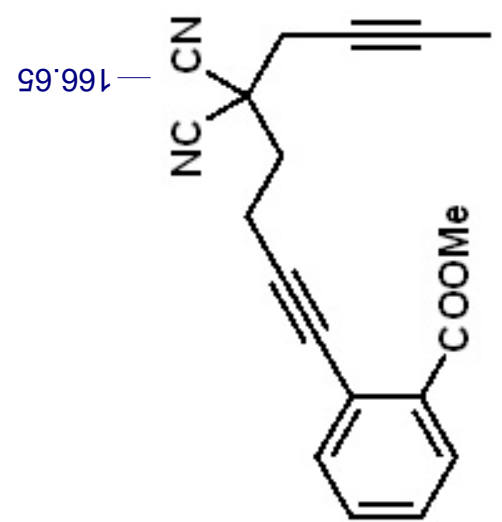




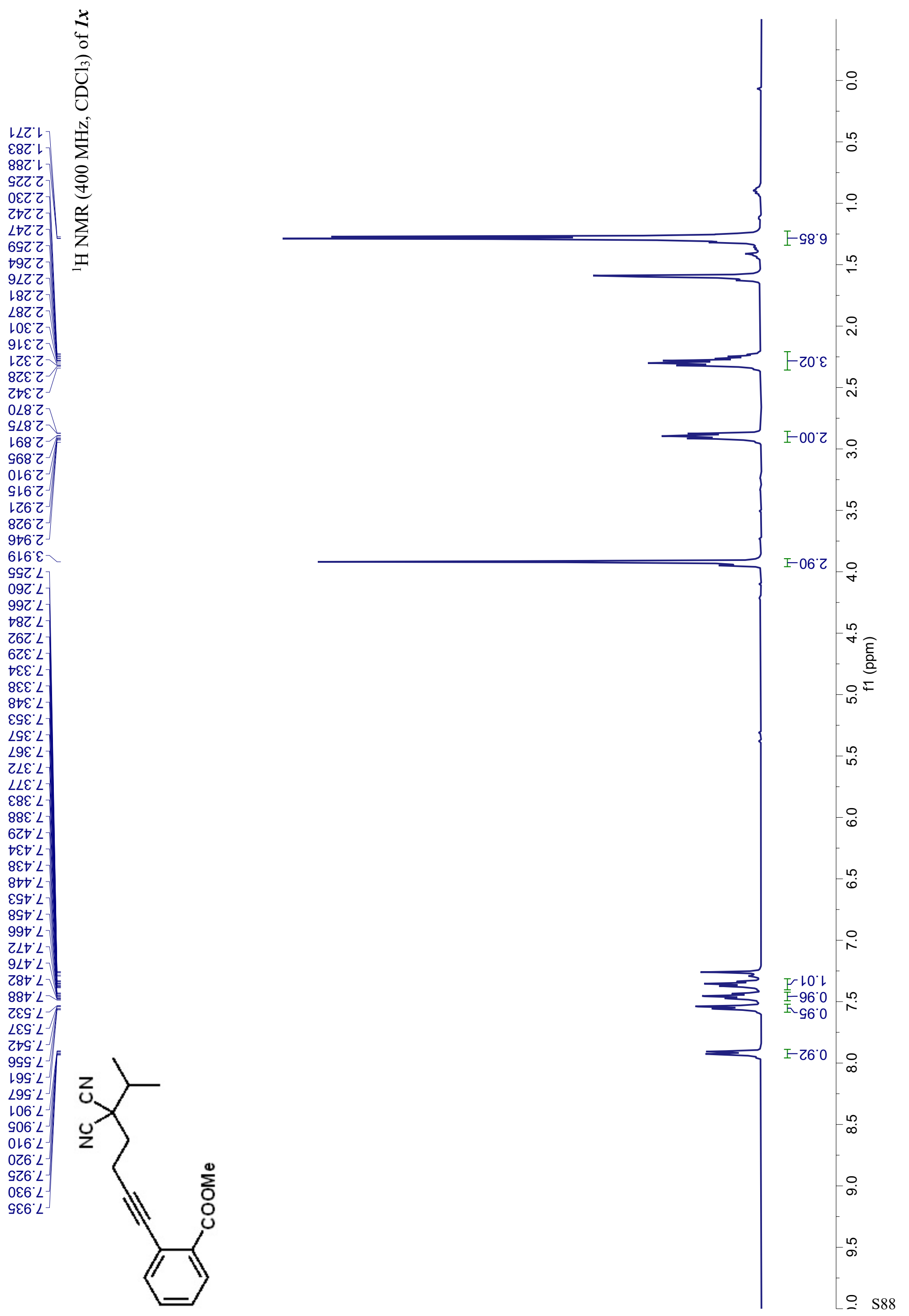




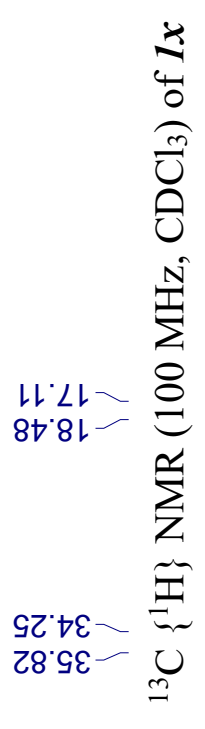

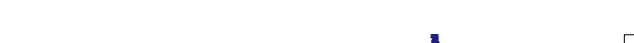

०๕`๕ฤ-

เE'Zs-

t8. $9 L$

$9 l^{\circ} \angle L-$

$8 D^{\circ} \angle L$

$\angle E^{\prime} 18$

$80 \cdot 16-$

ง9・เル-

ย9.๕Zレ

$00.8 Z 1$

t๑० $0 \varepsilon L$

$88^{\circ} L E L$

$\varepsilon 0$ 'Zع

เナ๋ยレー
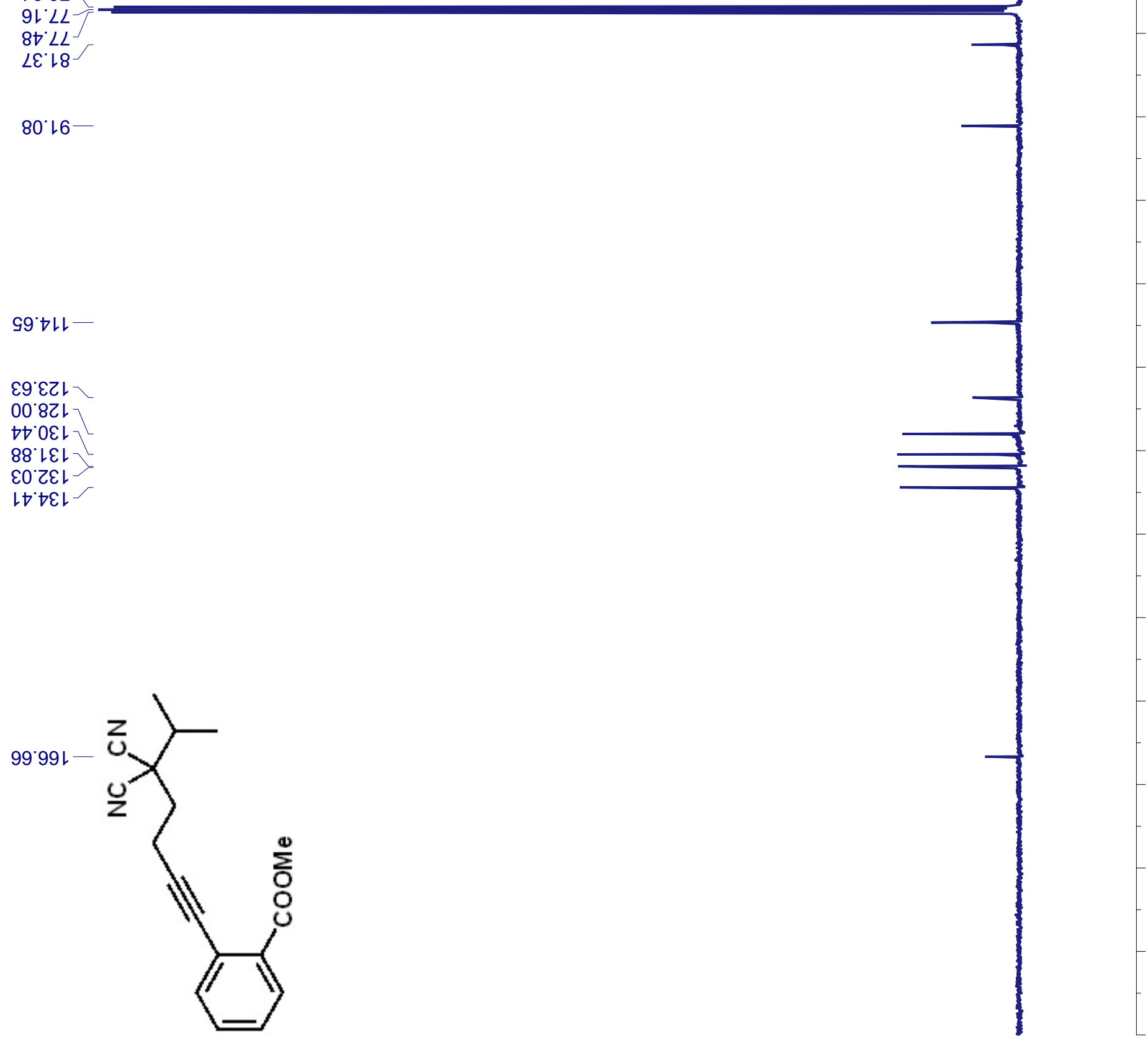

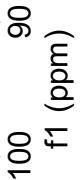

음

ิㅗ

오

움

움

r

8

옹

$\stackrel{\infty}{-}$

옴 


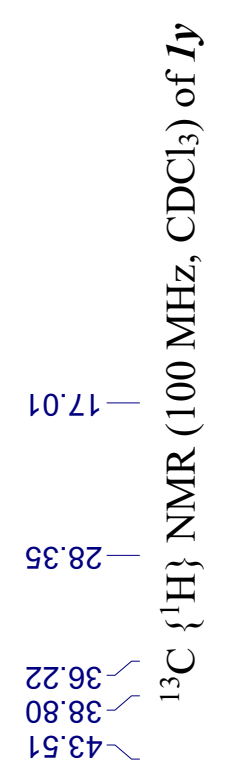

$78 \cdot 9 L$

$9 L^{\circ} L L$

$9 \varepsilon \angle L T$

$8 D^{\circ} L L$

E9. $18-$

ว8. 18

$\varepsilon)^{\circ} 06-$

て8・レレー

घ6 ZZレ-

96 LZL

$7062 \mathrm{~L}$

$\angle L^{\circ} 62 L$

$8 L^{\circ} 0 \varepsilon L$

เナ0EL

乙乙 LEL

8L'LEL-

$\neg 0^{\circ} \nabla \varepsilon L$

$\angle Z \nabla \varepsilon L$
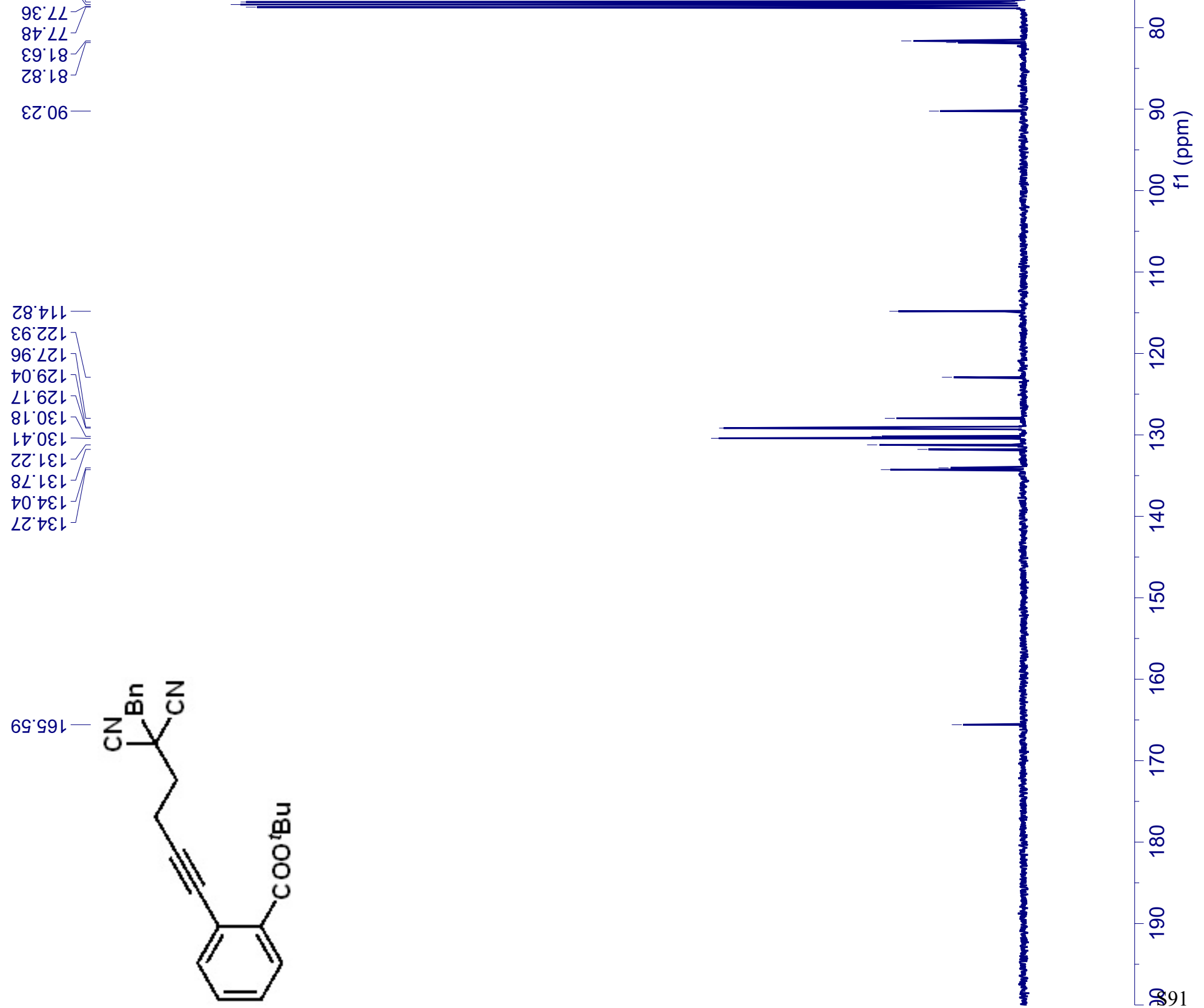
† $\mathcal{E}^{\circ} \varepsilon-$

$\nabla \nabla \varepsilon^{\circ} \dagger$

Z9E'

$08 \varepsilon^{\circ} \circ$

$86 \varepsilon^{\circ} \triangleright$

092 L

$\nabla \varepsilon \varepsilon^{\circ} L$

$\angle E \varepsilon^{\circ} \angle$

ESE $\angle$

$99 \varepsilon^{\circ} L$

ZLE' $\angle$

$9 \angle \varepsilon^{\circ} L$

$06 \varepsilon^{\circ} L$

$60 t^{\circ} \mathrm{L}$

8Lt L

$\checkmark Z \nabla^{\circ} L$

$\angle Z \nabla^{\circ} L$

乙๐た L

$\varepsilon \nabla \nabla^{\circ} L$

$9 \nabla t \angle$

$197^{\circ} \mathrm{L}$

G9t $L$

$\varepsilon \angle \nabla^{\circ} L$

$\varepsilon 8 \nabla^{\circ} L$

06t $\mathrm{L}$

$6 Z 9^{\circ} \mathrm{L}$

EES $L$

$6 \nabla G^{\circ} L$

ZSG' $L$

$016{ }^{\circ} \mathrm{L}$

EL6 L

6Z6 L $L$
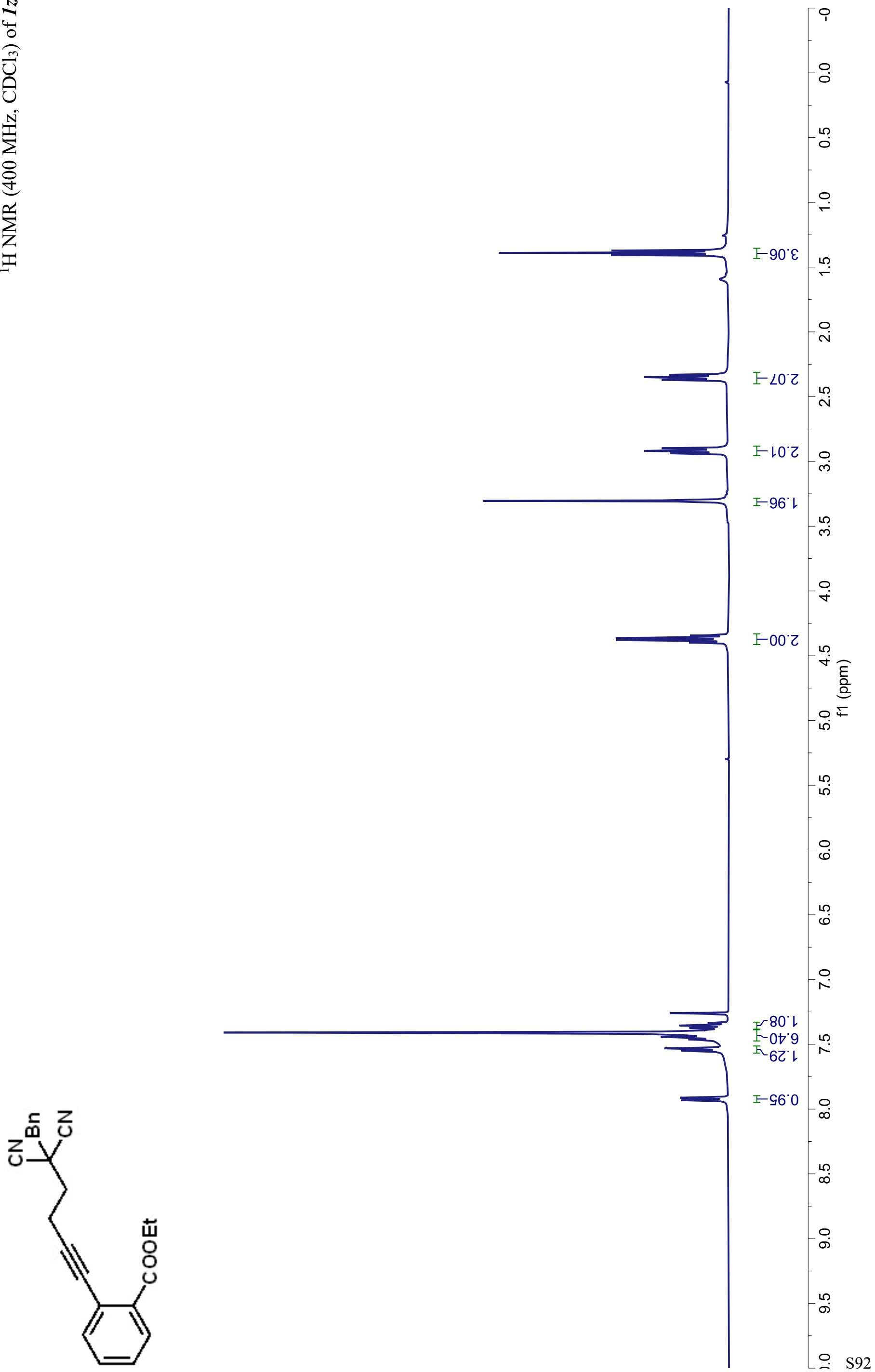

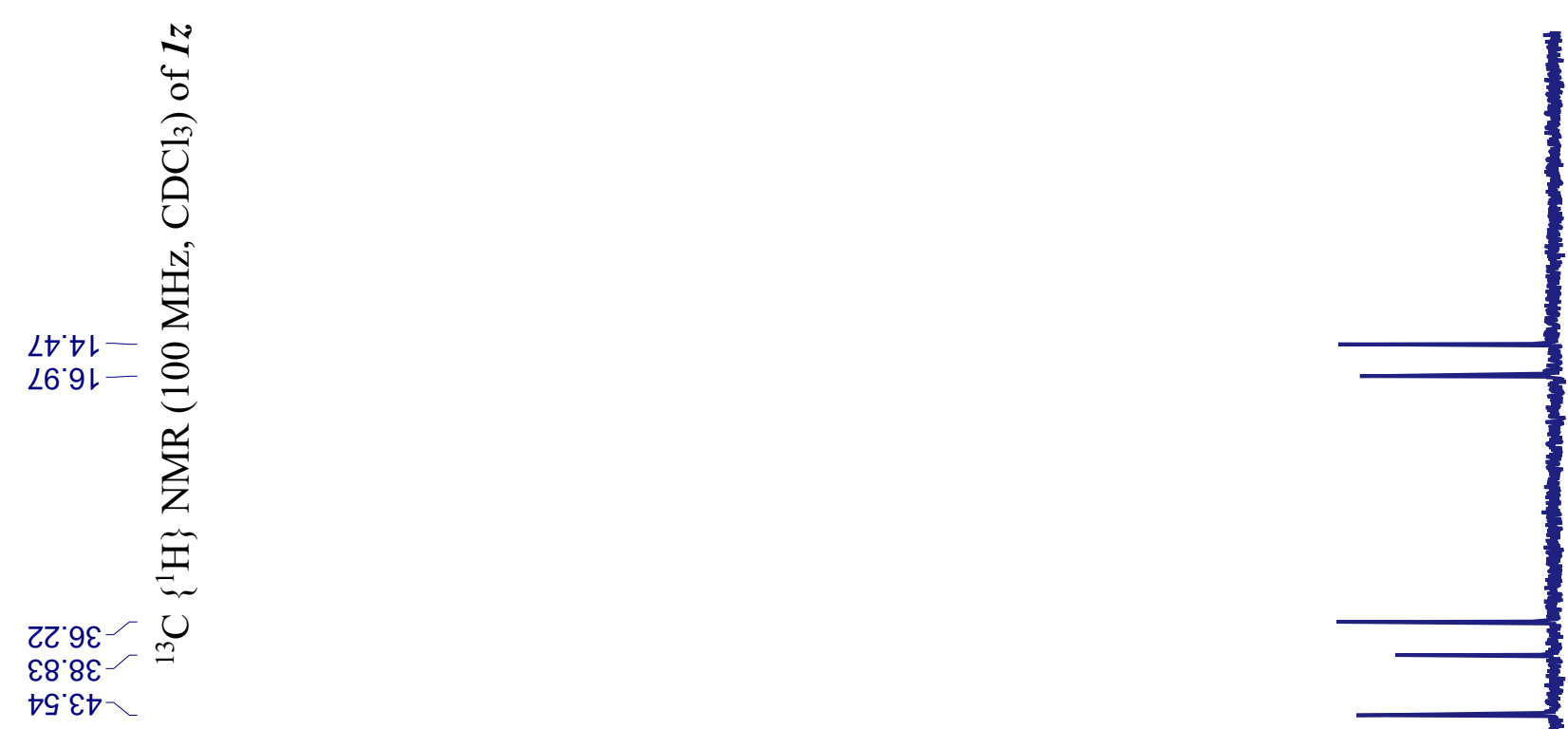

เว:19-

$78.9 L$
$9 L^{\circ} L L$

$87^{\circ} L L$

29.18

$\varepsilon\llcorner 06-$

†8・レレー

9† ย乙レ

L $0.82 \mathrm{~L}$

$90^{\circ} 6 \mathrm{ZL}$

8L6ZL

$8 \varepsilon^{\circ} 0 \varepsilon\llcorner$

$\varepsilon \nabla^{\circ} 0 \varepsilon\llcorner$

EL'LEL-

$\neg 8$ 'LEL-

O† ZEL

$9 \varepsilon^{\prime} \nabla \varepsilon\llcorner$

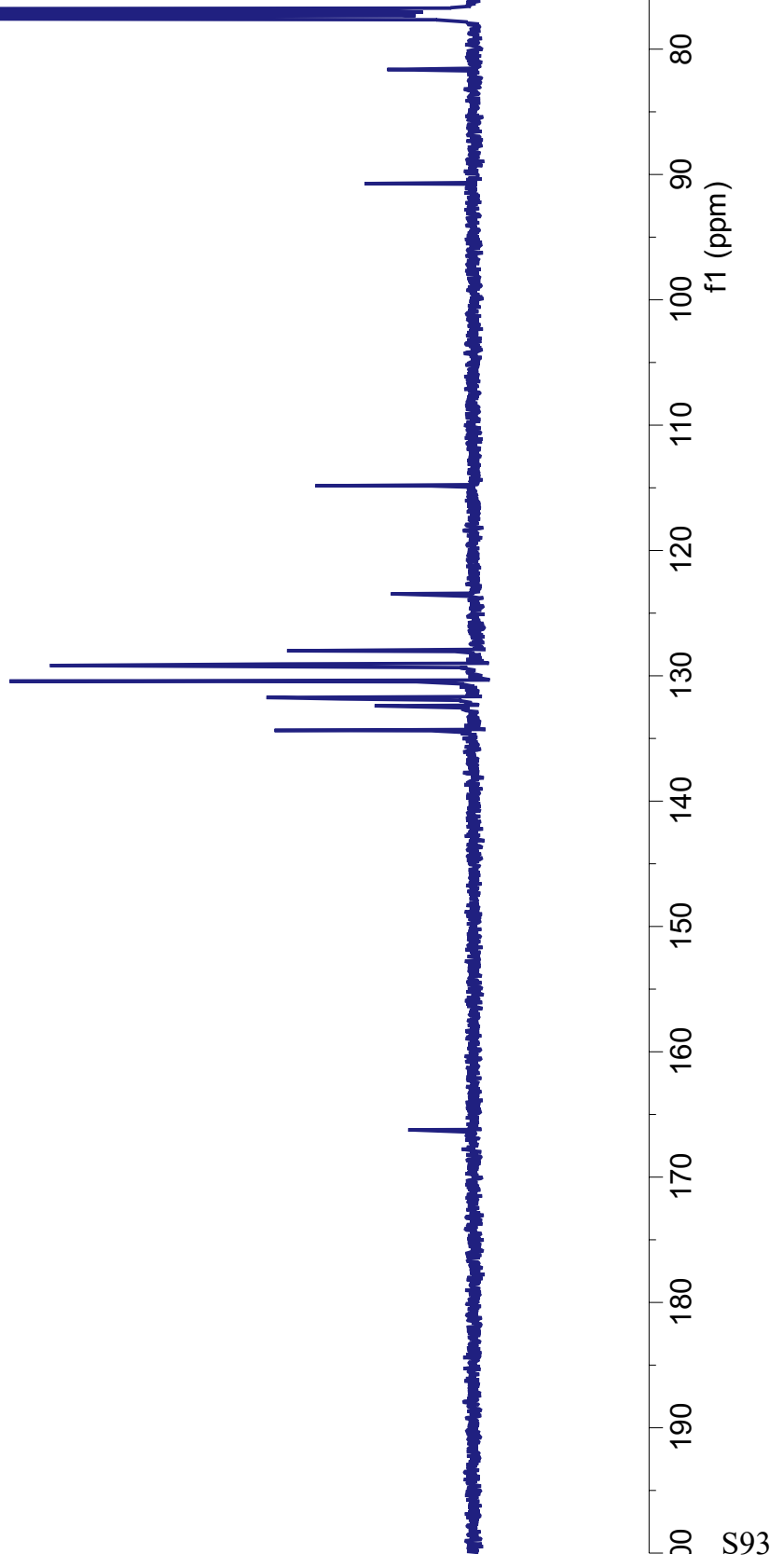




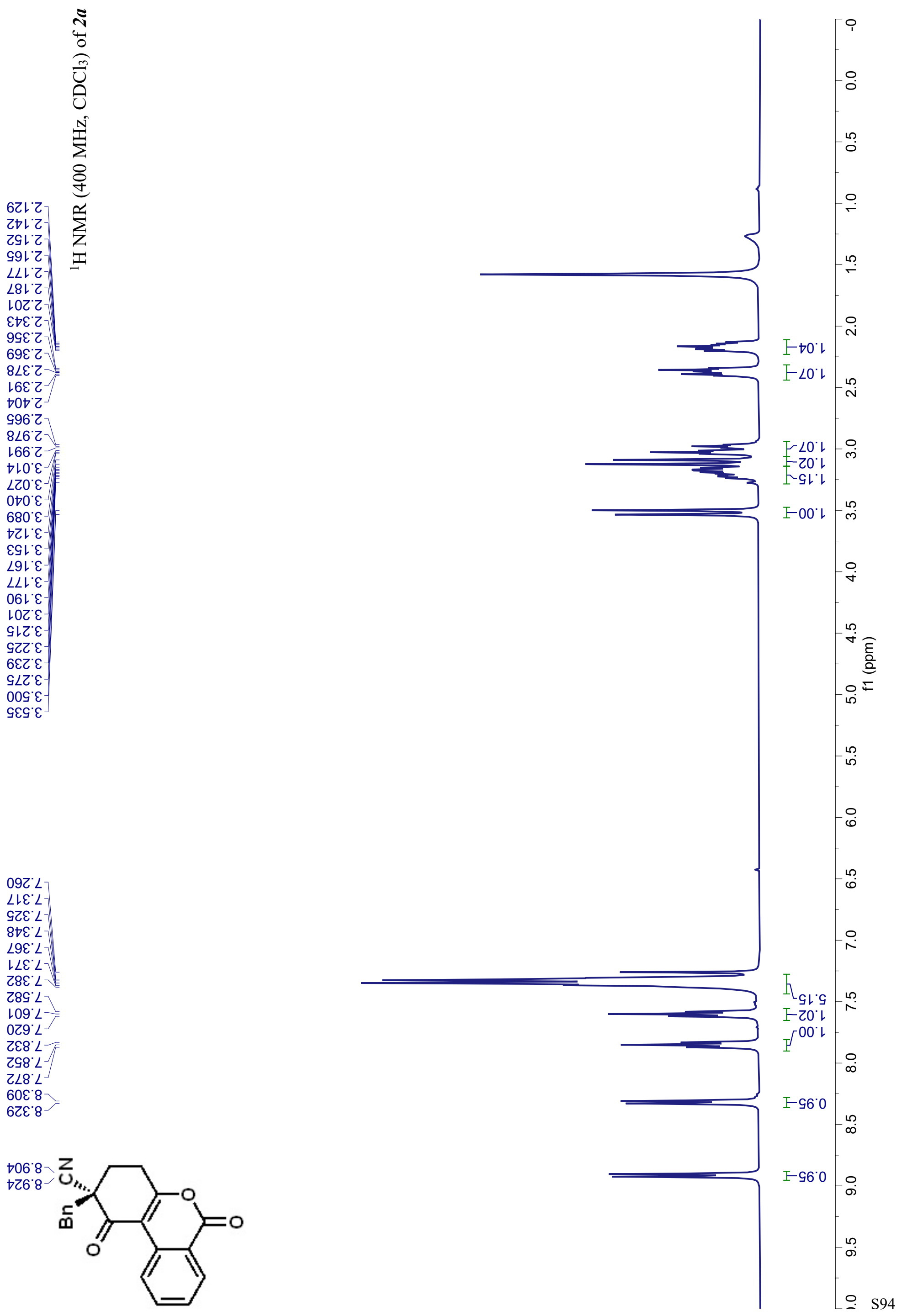




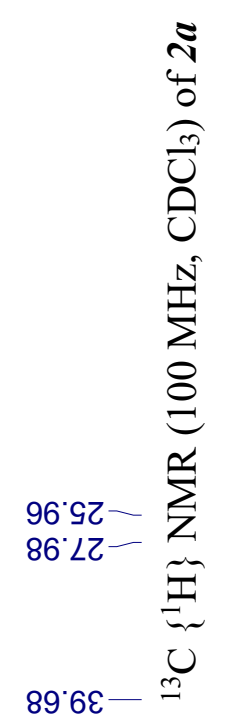

$78^{\circ} 9 L$

98ㅇㄴ -

82.8ル

10.0ZL

98'G乙L

EL $8 Z$ L

96.8ZL

$\neg \varepsilon^{\prime} 6 Z L$

GLOEL

LG'0EL

$\varepsilon \varepsilon$ $\varepsilon \varepsilon\llcorner-$

ह8 $\varepsilon \varepsilon\llcorner$

$6 L^{\circ} 9 \varepsilon \mathrm{L}$

L969L-

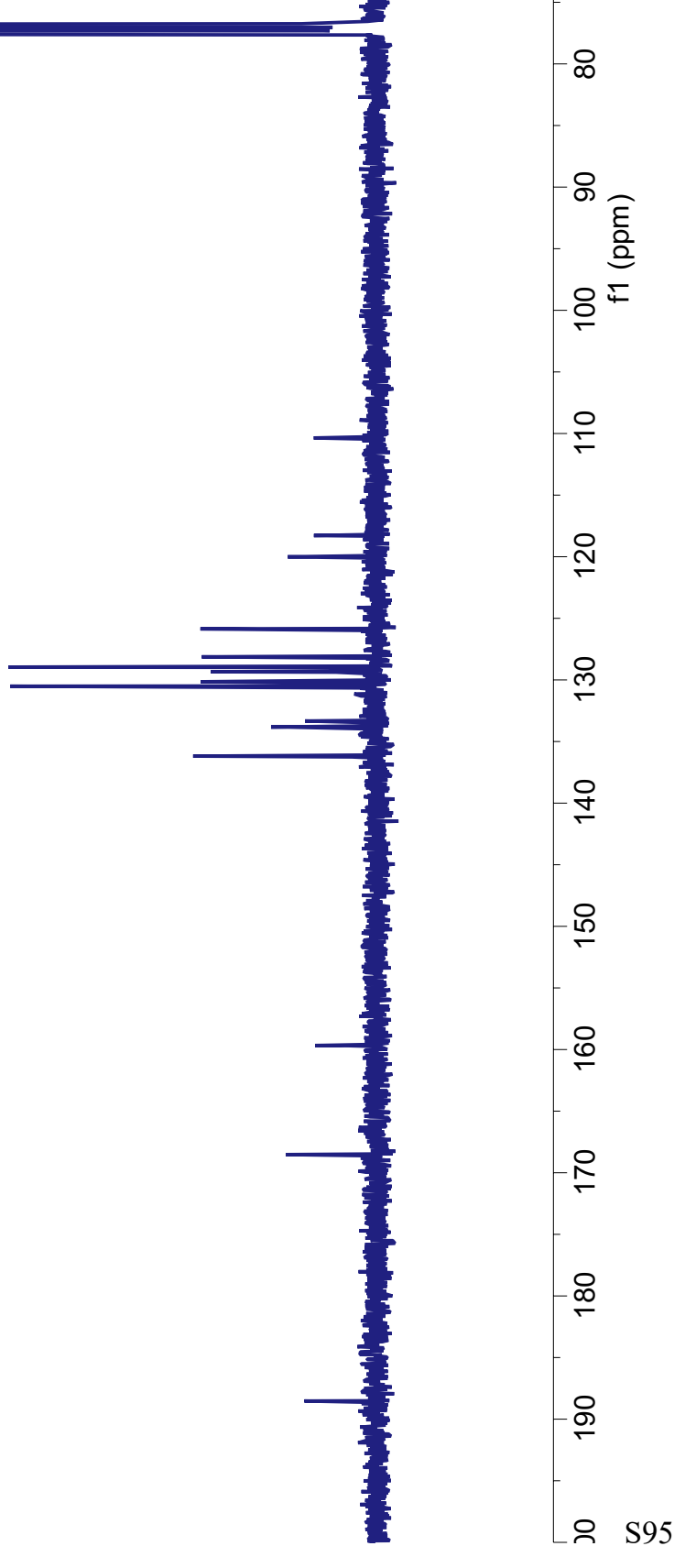

Z9.891-

EG'88 -

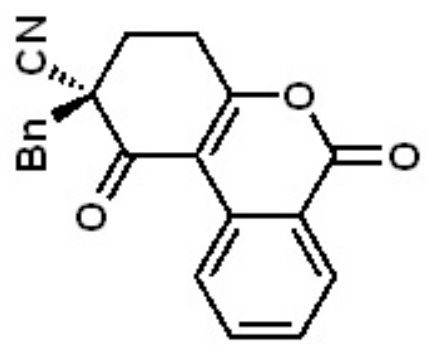




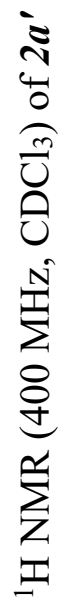

$\angle L \varepsilon^{\prime} Z$

$16 \varepsilon^{\circ} \mathrm{Z}$

$\angle 6 \varepsilon^{\prime} \mathrm{Z}$

†0七

டレナ乙

$8 \varepsilon 6^{\circ} \mathrm{Z}$

IS6 2

$896^{\circ} \mathrm{Z}$

†96 $\mathrm{Z}$

$8 \angle 6^{\circ} \mathrm{Z}$

$\angle L Z^{\circ} \varepsilon-$
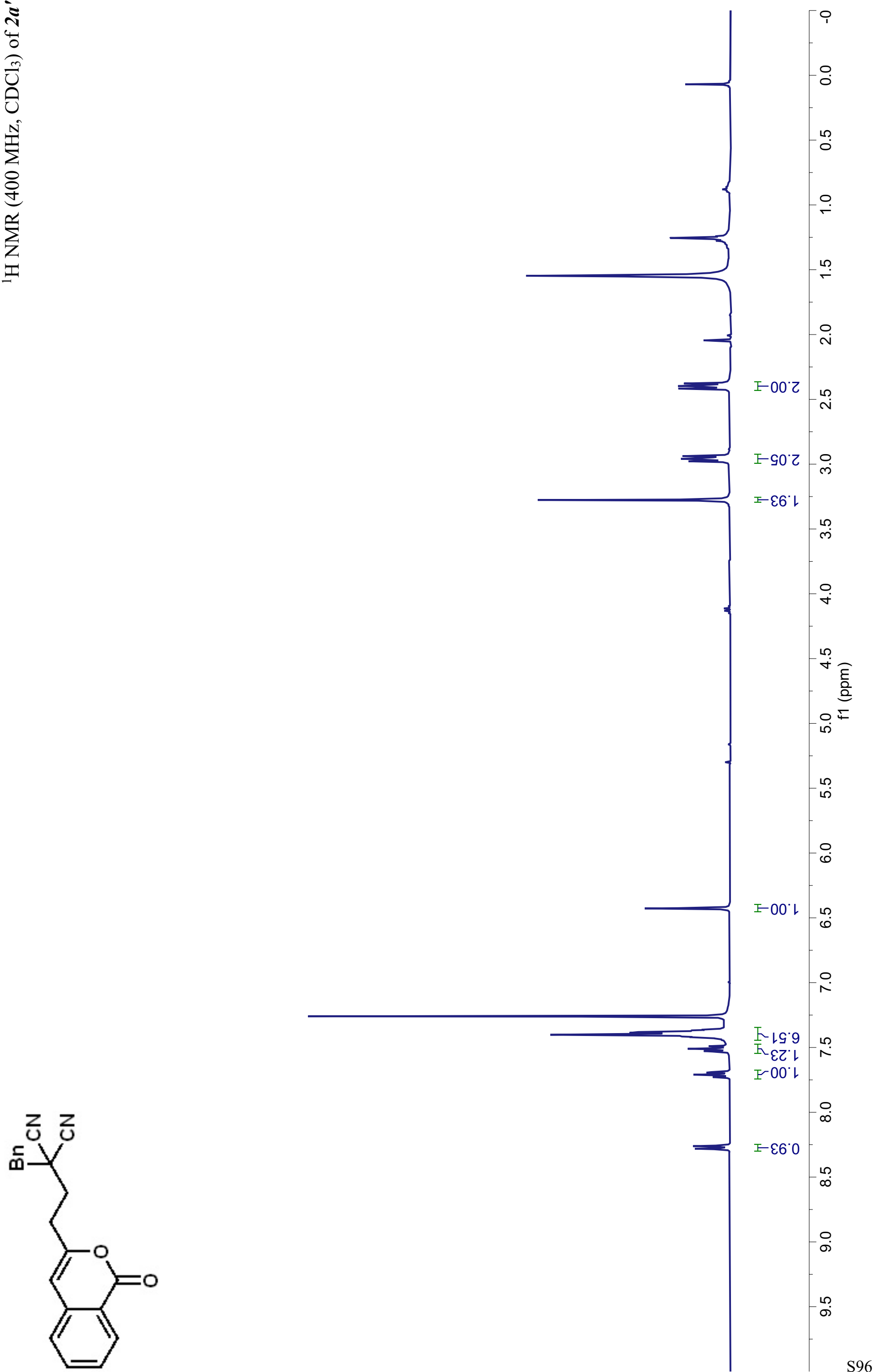


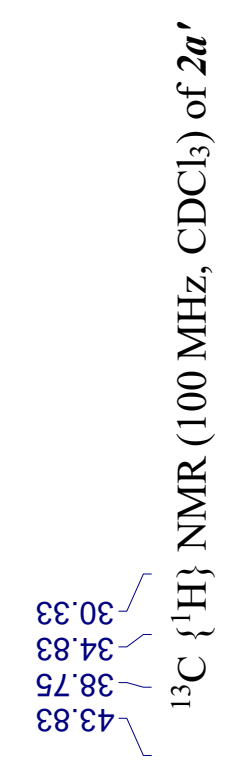

เย.9Oเ-

8ட゚レレー

6S.0ZL

09.9ZL

Z9.8ZL

9l6Zl

งZ'6ZL

98.6ZL

$6 \varepsilon^{\circ} 0 \varepsilon\llcorner$

$09^{\circ}\llcorner\varepsilon L$

OZ $G E L$

L8 $9 \varepsilon$ L
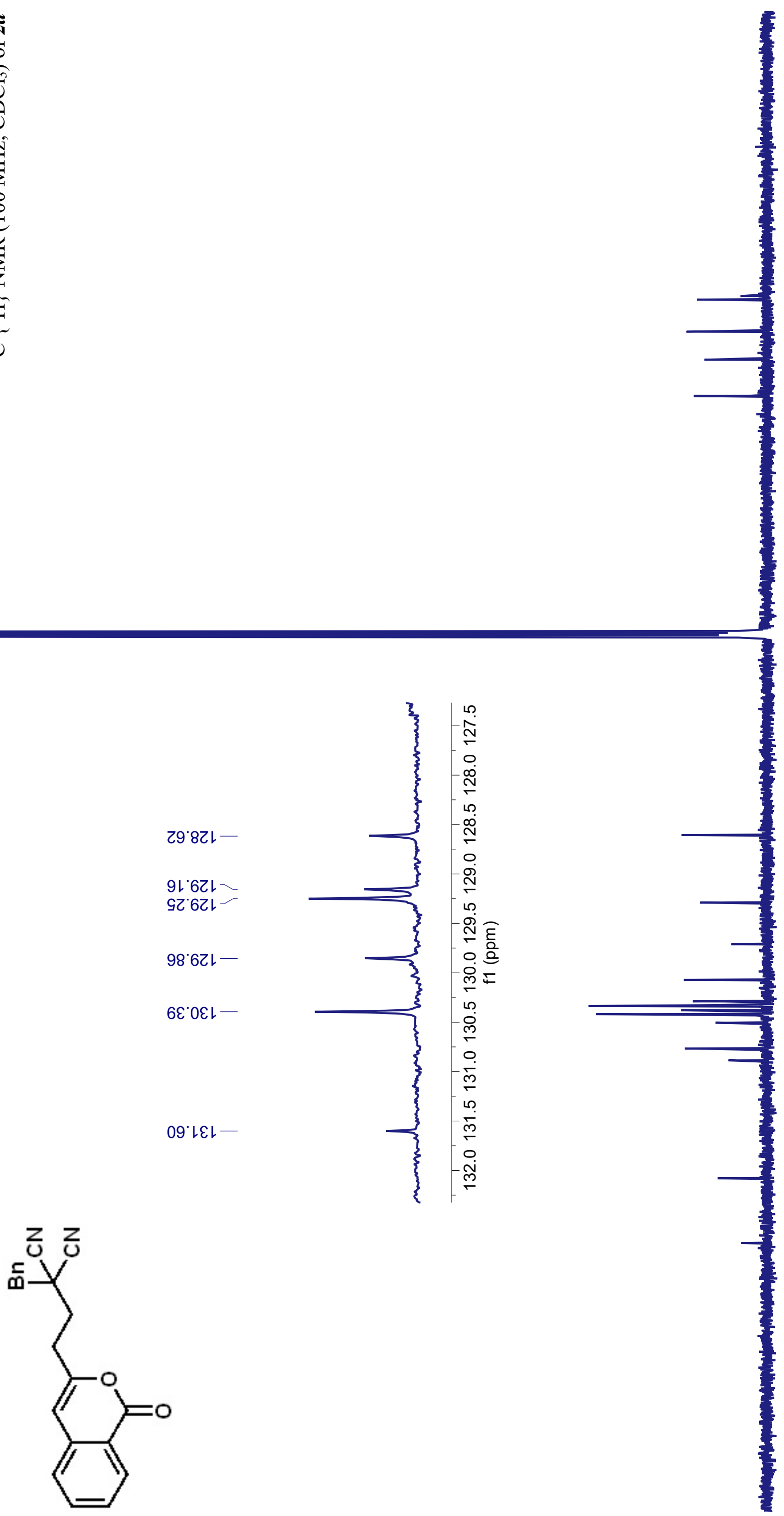

เカว9レー 


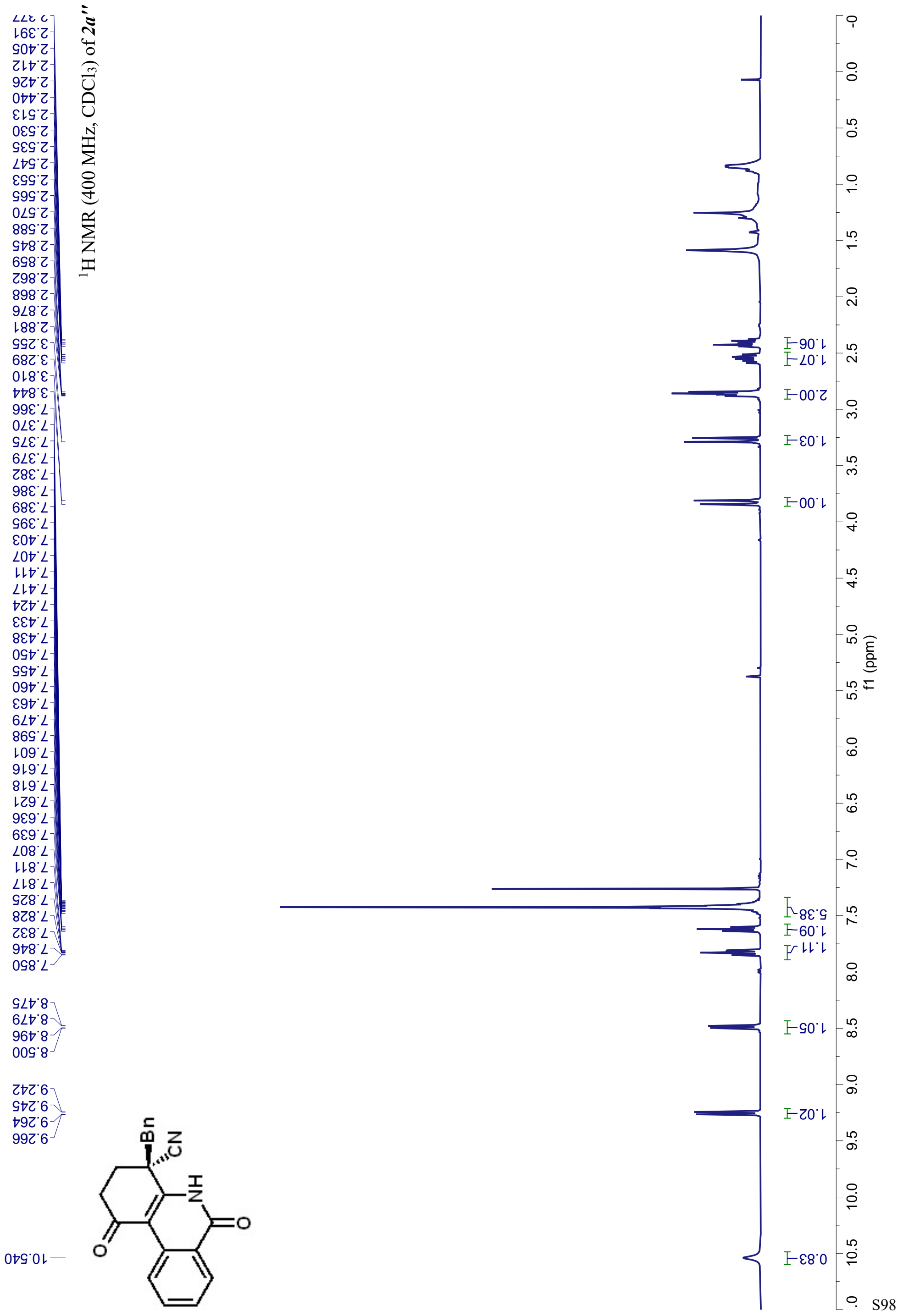




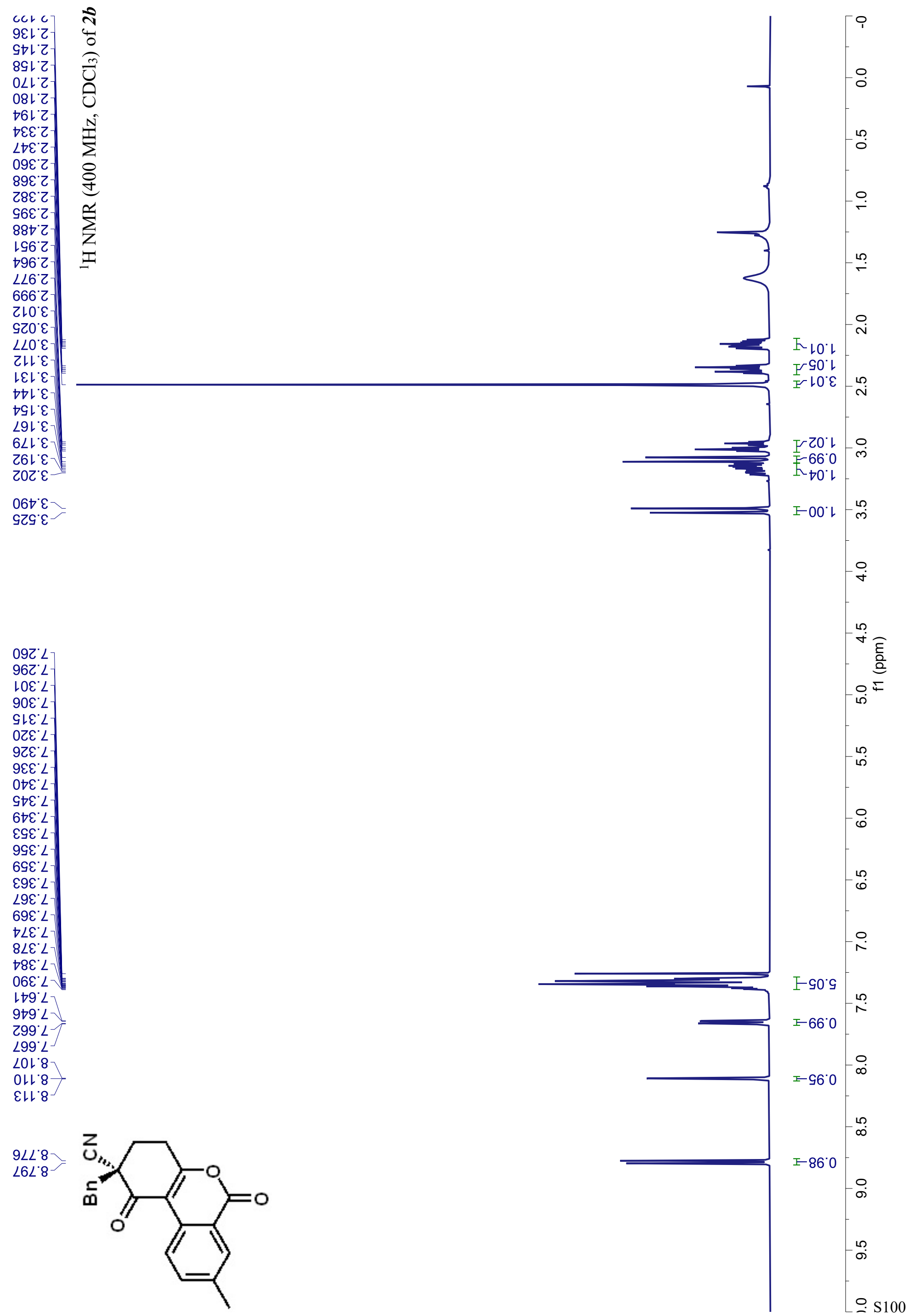




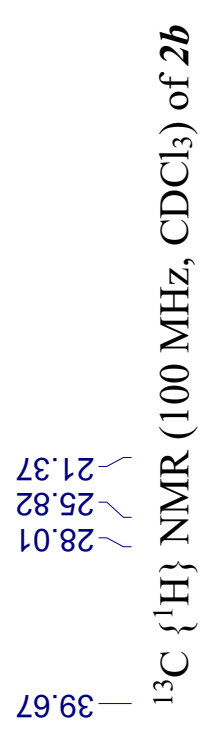

$\varepsilon 0^{\circ} 6 t-$
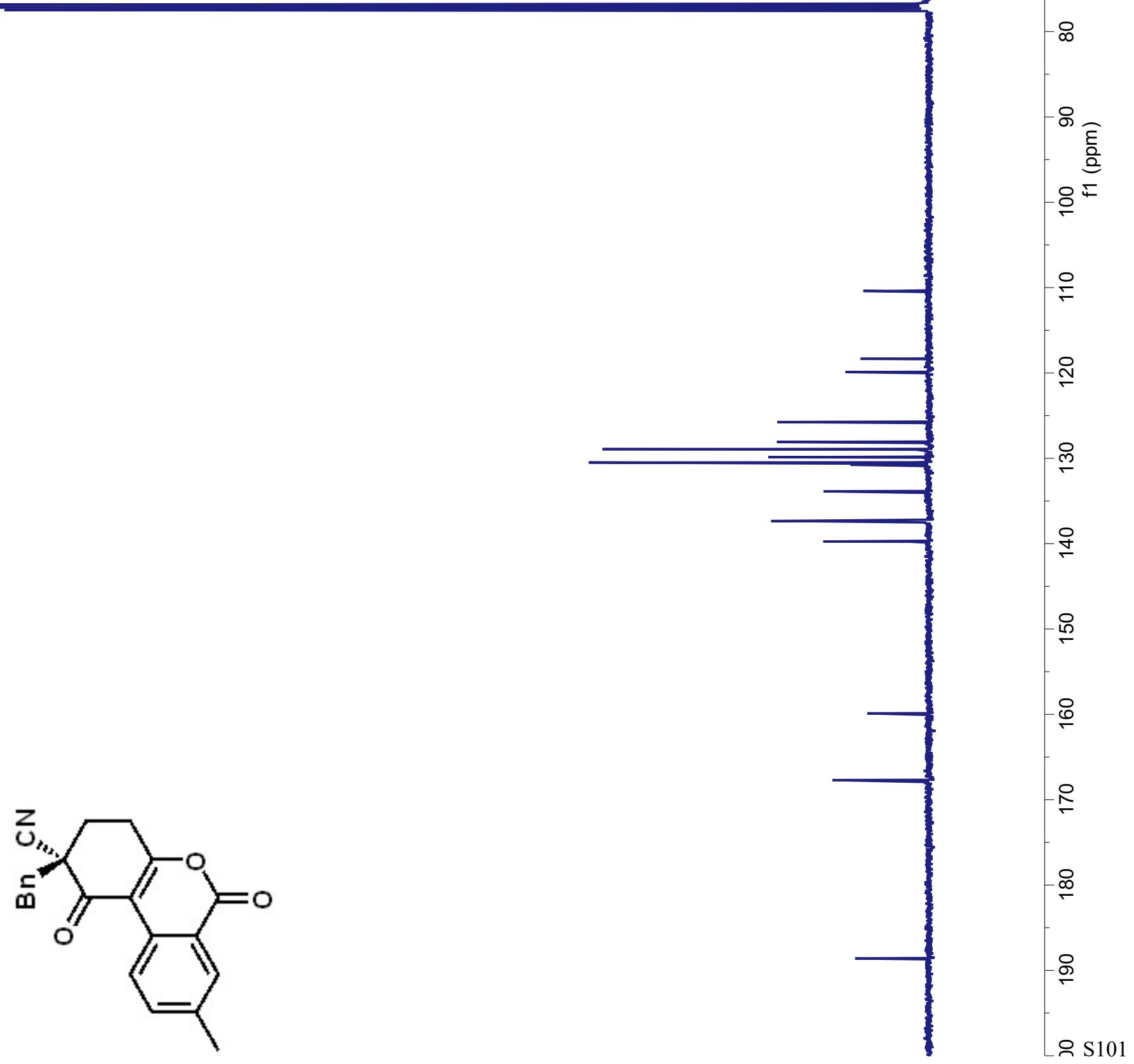


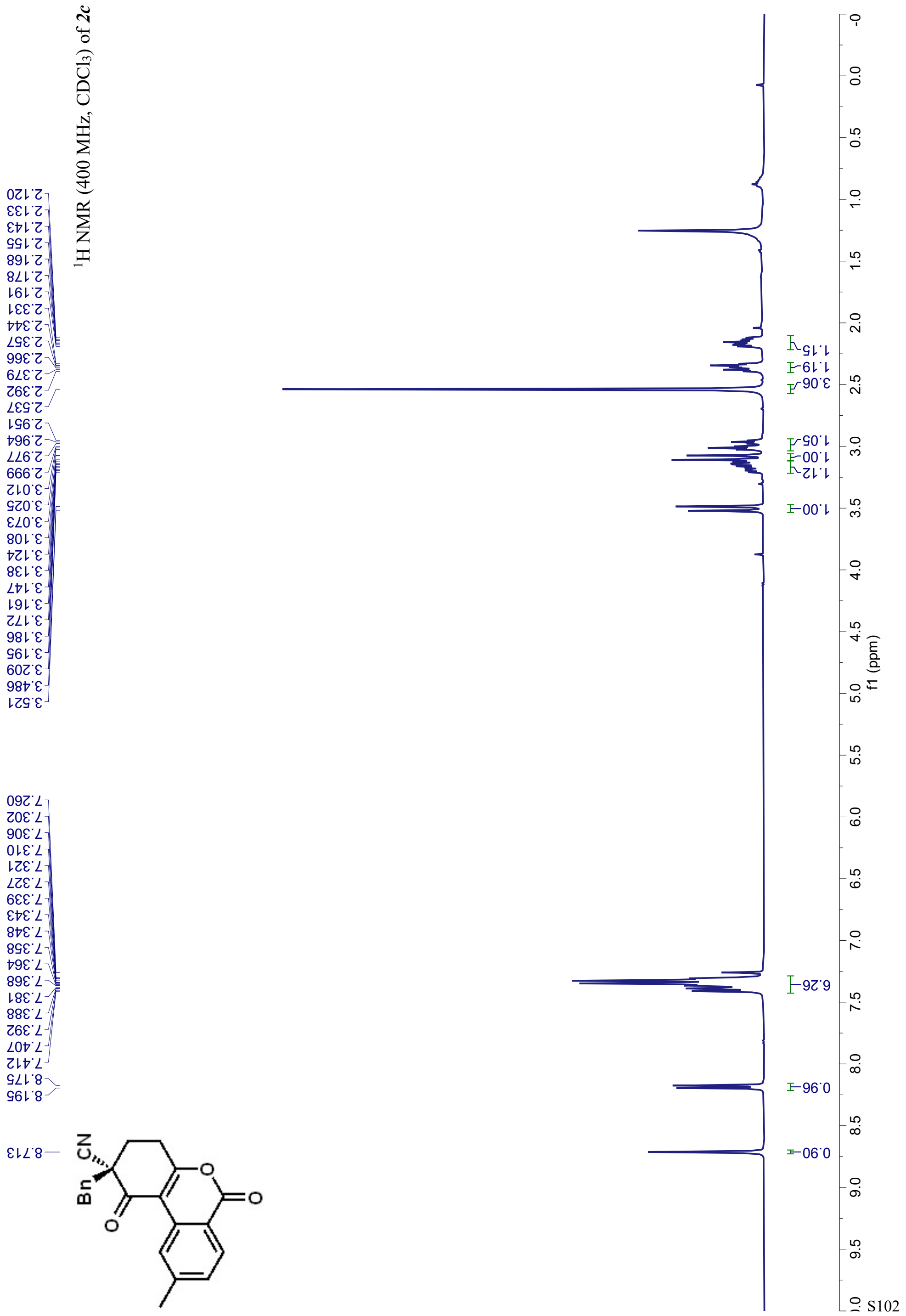




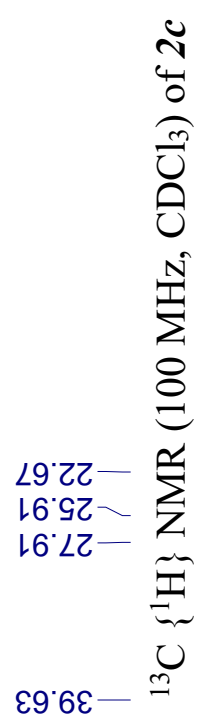

$80 \cdot 67-$

$78^{\circ} 9 L$

$9 L^{\circ} L L \frac{}{5}$

เZ゚Oレー

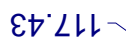

เE8เレ

เ6.92L

60.8Z -

Z6.8ZL

$600^{\circ} 0 \varepsilon \mathrm{L}$

8t.0عL

$\angle 乙 \cdot \varepsilon \varepsilon\llcorner$

$\varepsilon 8^{\circ} \varepsilon \varepsilon\llcorner$

99くャレー

69.881

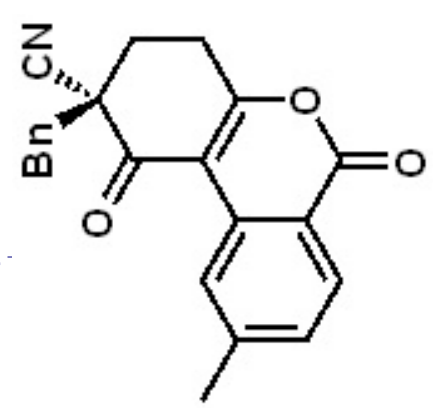

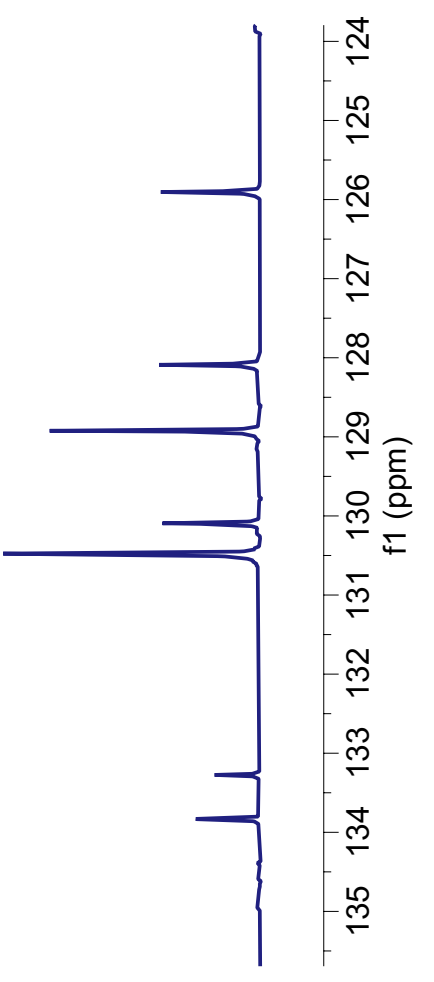


$1 \varepsilon^{\circ} \varepsilon-$

$09 Z^{\circ} L$

$86 Z^{\prime} L$

$\varepsilon 0 \varepsilon^{\circ} L$

$80 \varepsilon^{\prime} L-$

$\angle L E \angle$

ZZE' $\angle$

$6 Z \varepsilon^{\circ} L$

$6 \varepsilon \varepsilon^{\circ} \angle-$

$\varepsilon \sqcup \varepsilon$

$8 \nabla \varepsilon^{\prime} L$

$\varepsilon \subseteq \varepsilon^{\circ} L$

$09 \varepsilon^{\circ} L$

Z9E $L$

$\angle 9 \varepsilon^{\circ} \angle$

ZLE $L$

$\angle L \varepsilon^{\circ} \angle$

乙8६ $\angle$

$88 \varepsilon^{\circ} L$

$96 \varepsilon^{\circ} L$

$\rightarrow 0 t^{\circ} \mathrm{L}$

6 Lt L

$9 Z+\angle$

OZL $L$

$\angle Z L L$

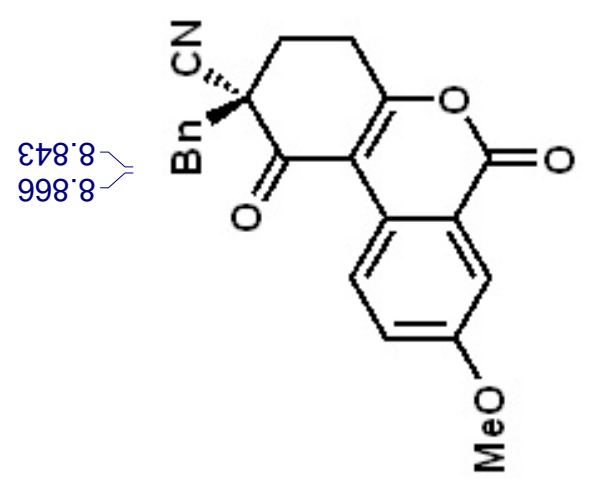

$\stackrel{\circ}{ }$

r

F+6.9

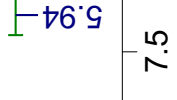

I $68^{\circ} 0$

$\stackrel{\circ}{\infty}$

$\stackrel{\infty}{\infty}$

I Z6. 0 


$78^{\circ} 9 L$
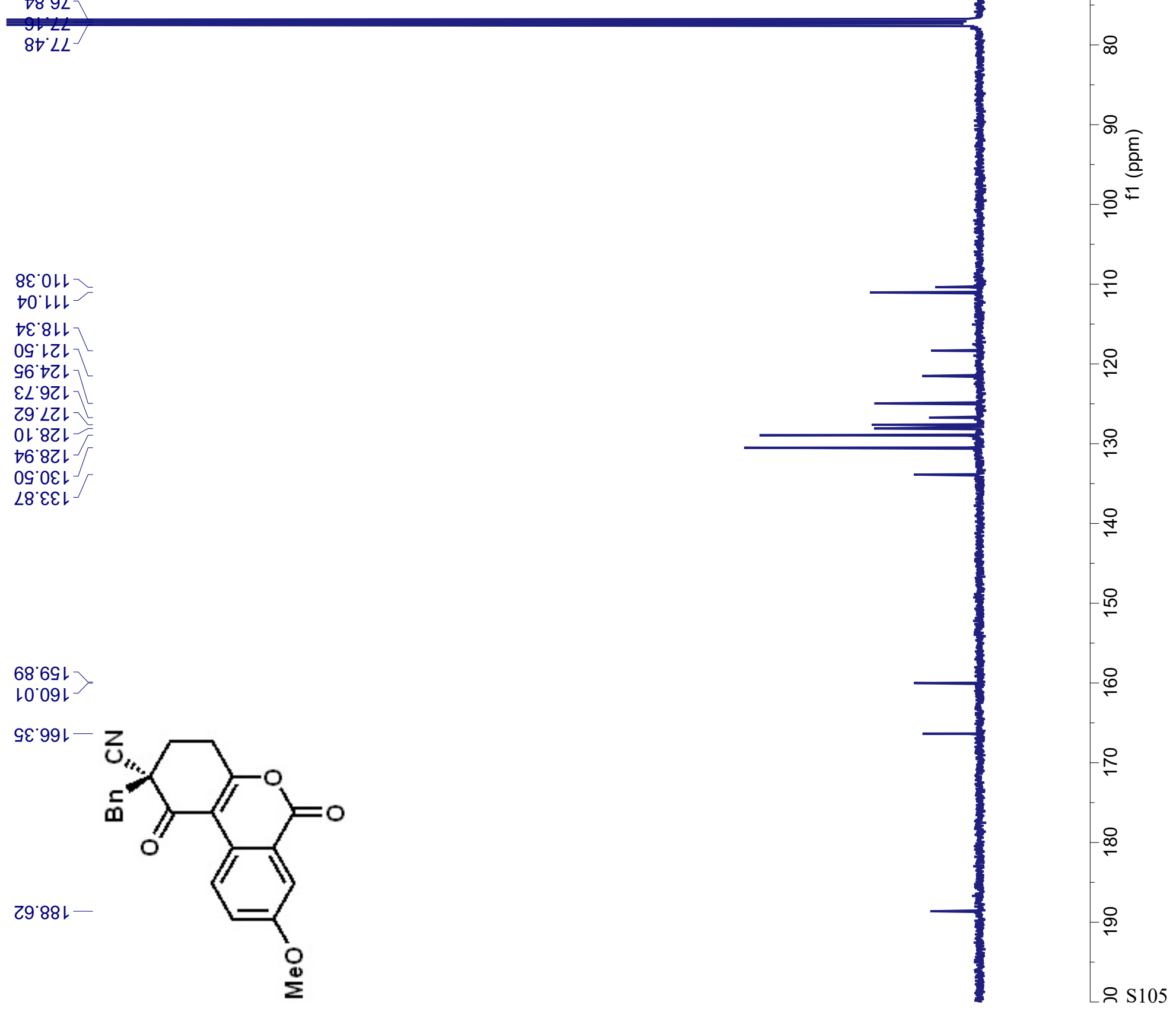


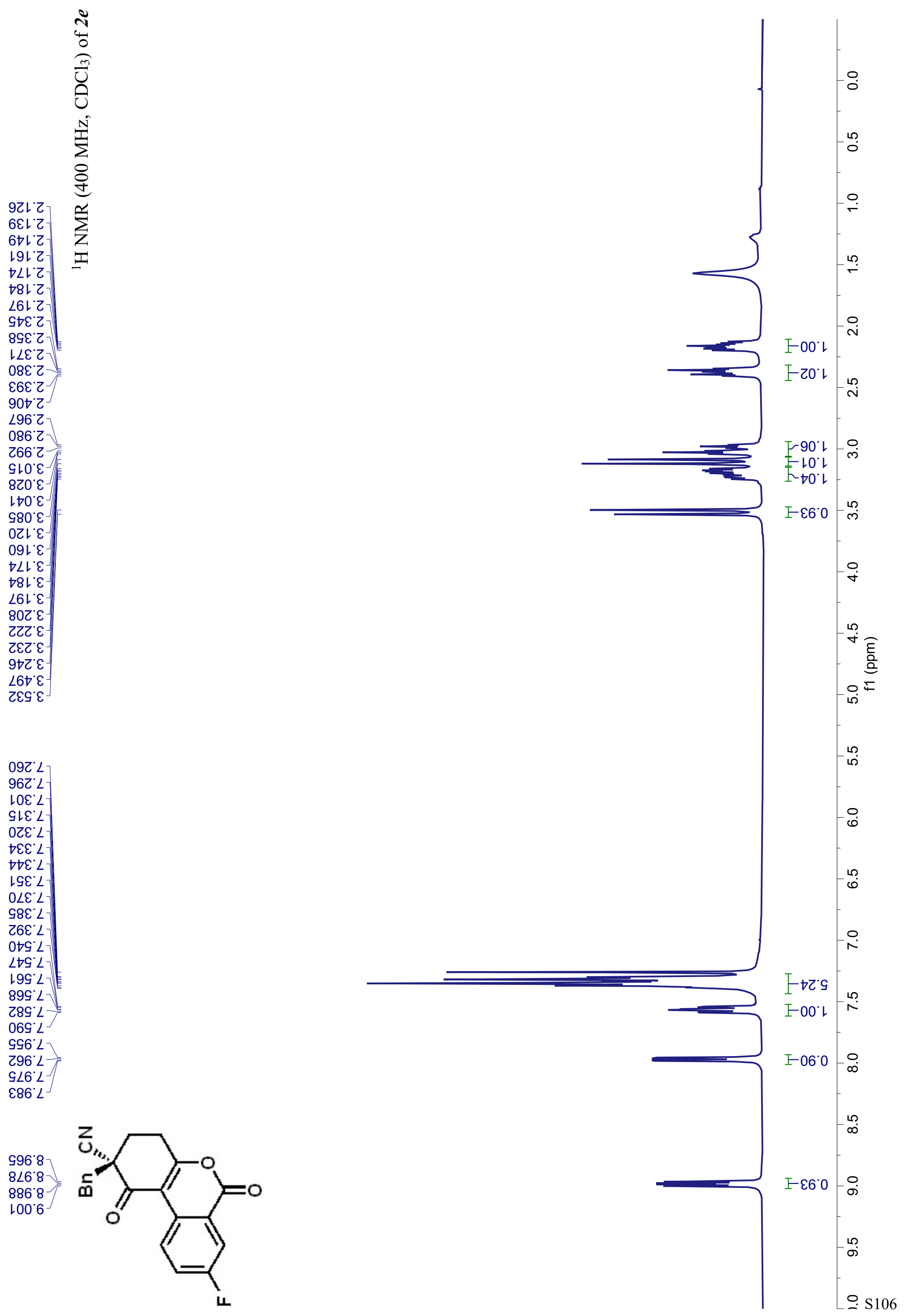




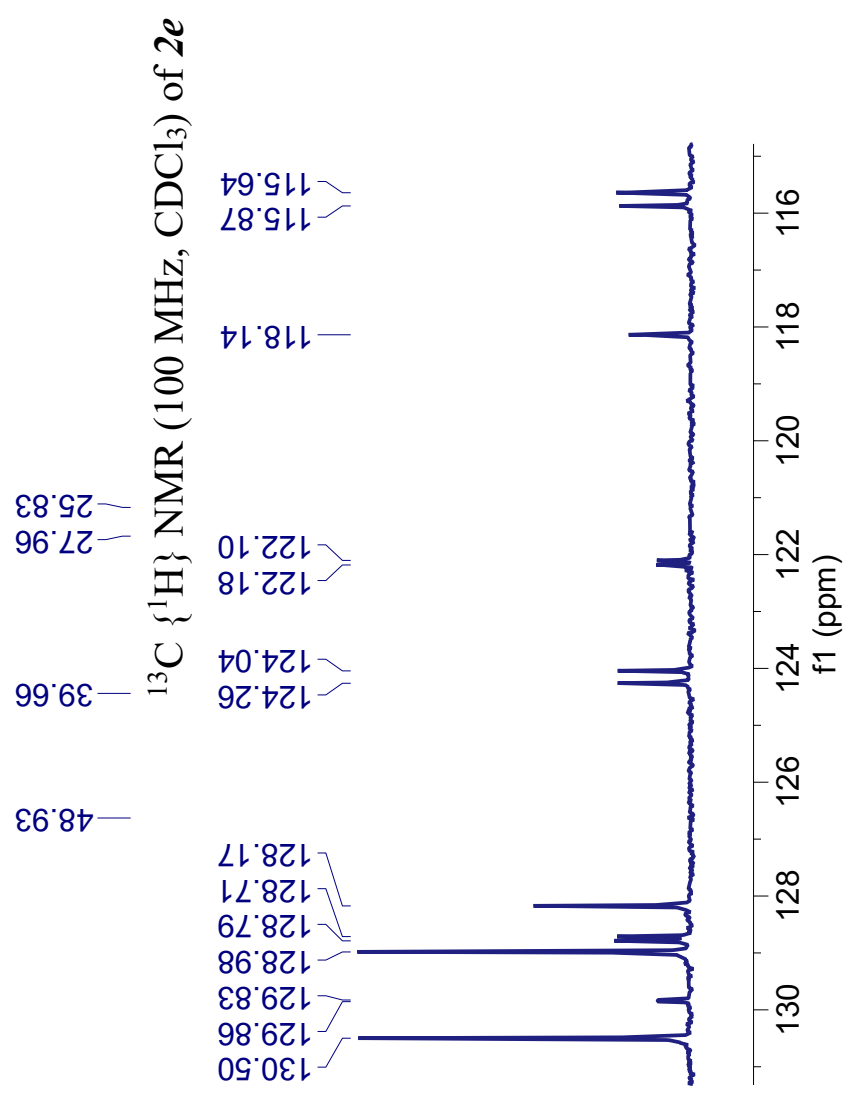

96.60L†9.9L L8GIㄴํレー 0レてZL

8レててレ $\checkmark 0^{\circ} \nabla C L$

$9 \nabla^{\circ} \nabla Z L$

$\angle L 8 Z L$

LL'8ZL

6L $8 Z L$

86.8ZL

ع8 6 Z

98.6Z

OS.0EL

L $\angle E \mathcal{E}$.

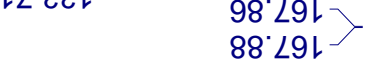

LL'8GL

08 .85

90 เ9L

$\angle G^{\prime} \varepsilon 9 \mathrm{~L}$

$98^{\circ} \mathrm{Lgl}$

88 ' 291

$97^{\circ} 881-$

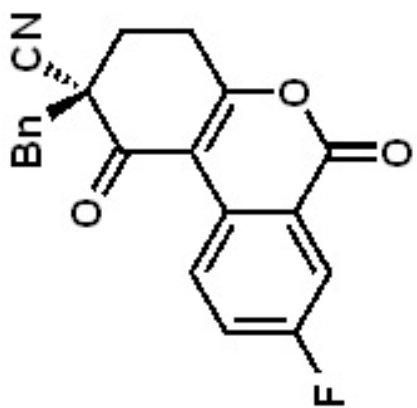

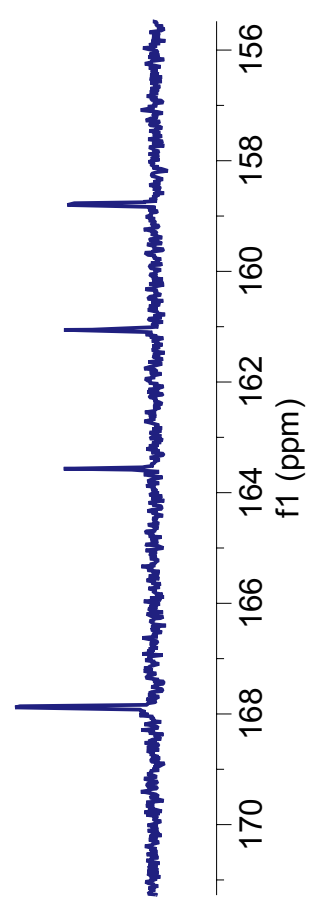

$-\infty$

옹

$\frac{\hat{\varepsilon}}{2}$
을 $^{\frac{2}{2}}$

은

ํำ

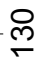

움

움

$\stackrel{8}{\circ}$

$-6$

온

$\stackrel{\infty}{\circ}$

옴 


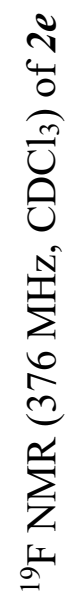

9ZL $80 \mathrm{~L}$

ZLL'80L-

$\mathrm{D} 0 \mathrm{~L}^{\circ} \mathrm{BO} \mathrm{L}$

$069^{\circ} 80 \mathrm{~L}$

$\varepsilon 89.80 \mathrm{~L}-$

$699^{\circ} 80$ L-
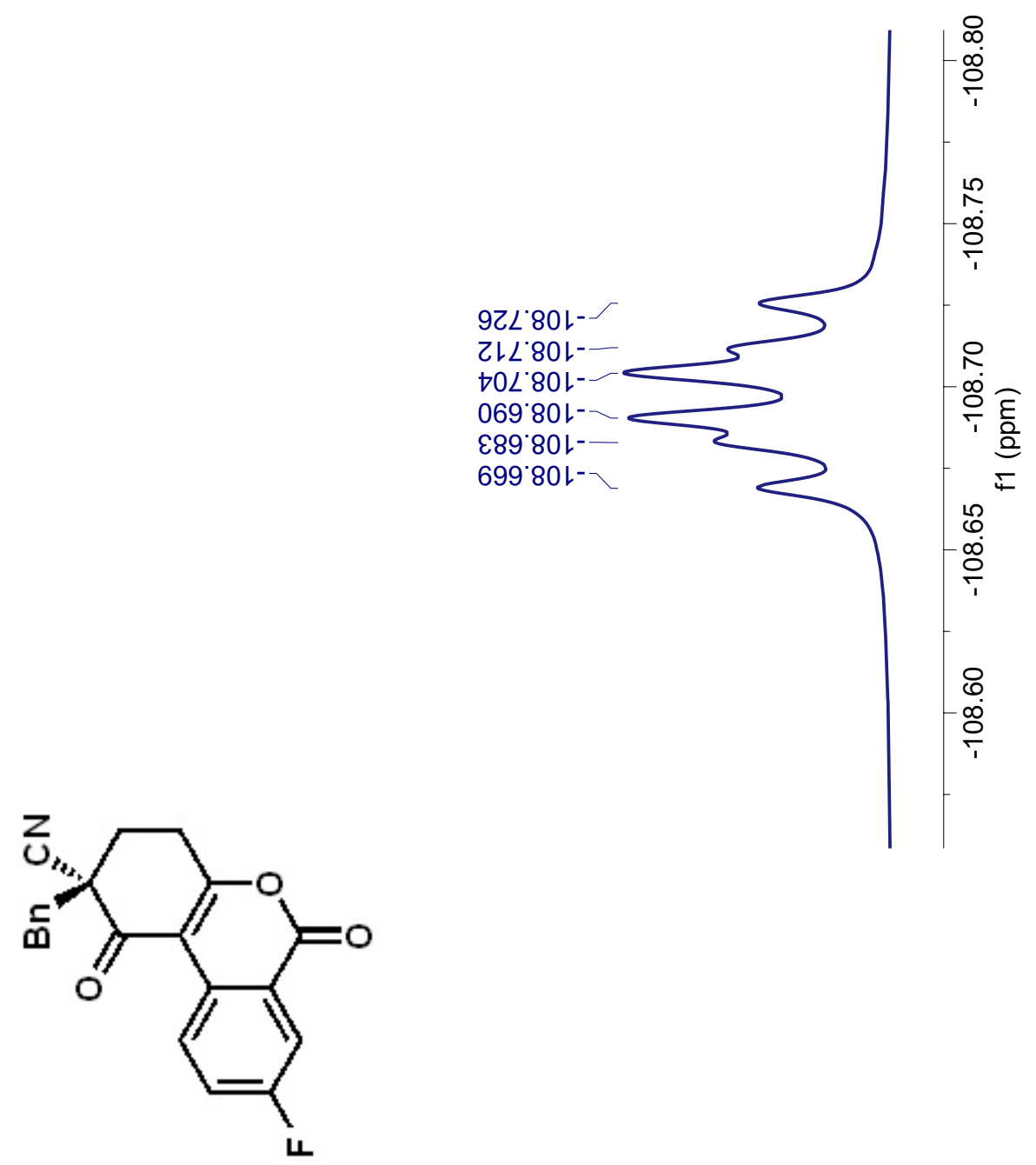


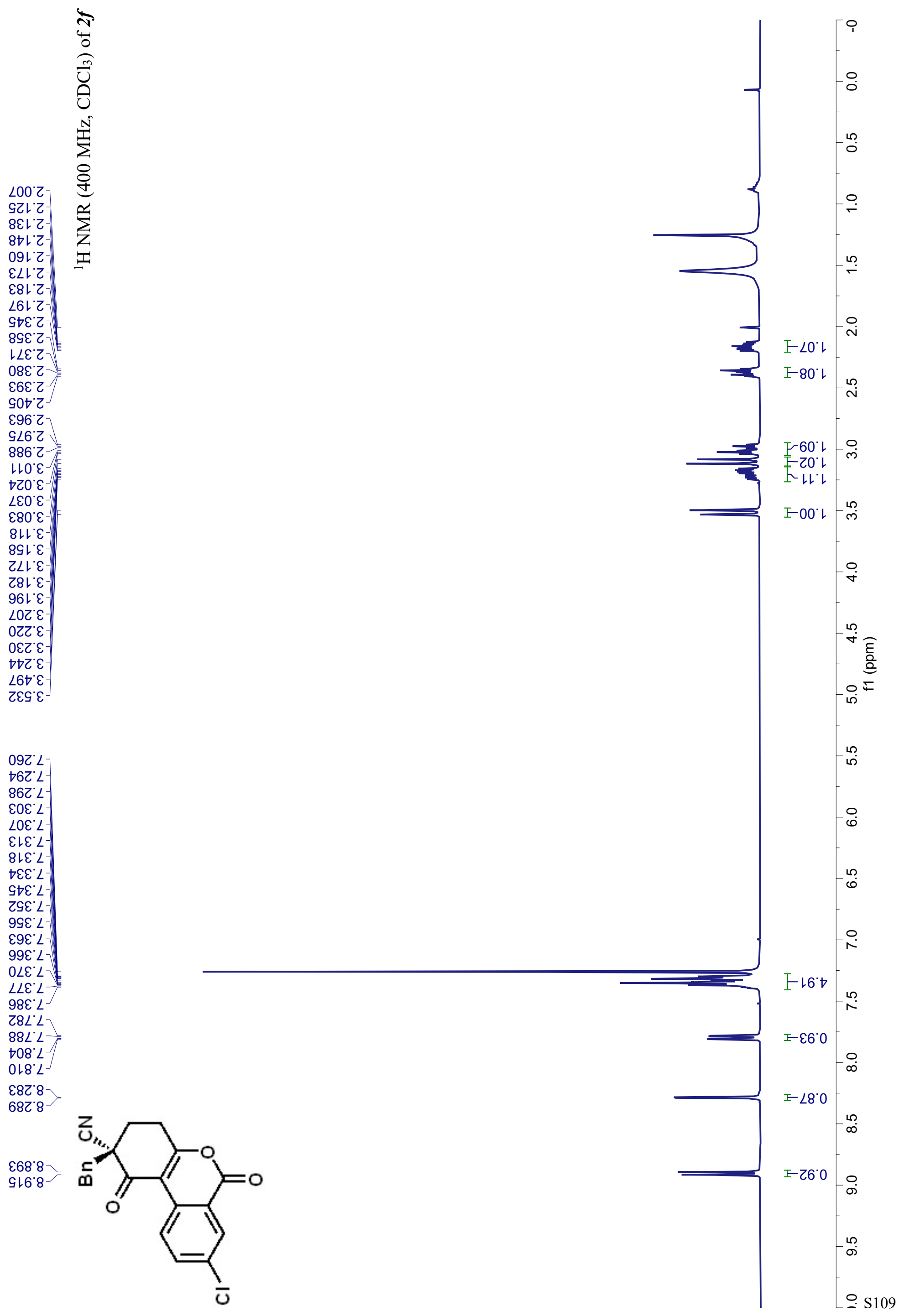




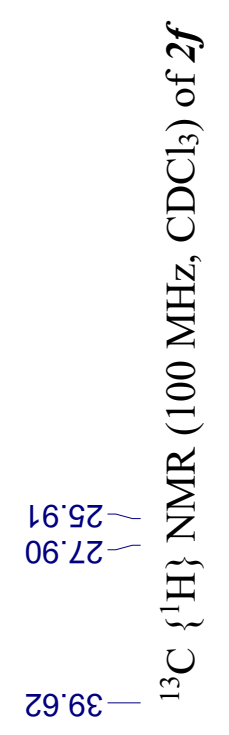

1

$76.87-$

$78{ }^{\circ} 9 L$

$9 L^{\circ} L L$

68.60 -

608ルー

97゙ルレー

$09^{\circ} \angle Z L$

$\angle L 8 Z L$

$86.8 Z$ L

$87^{\circ} 6 \mathrm{Z}$

$87^{\circ} 0 \varepsilon t$

$0 L$ ' $E L$

$\angle 9^{\circ} \varepsilon \varepsilon L$

St $G \varepsilon L$

$\nabla \varepsilon \cdot 9 \varepsilon\llcorner$

†G'8GL -
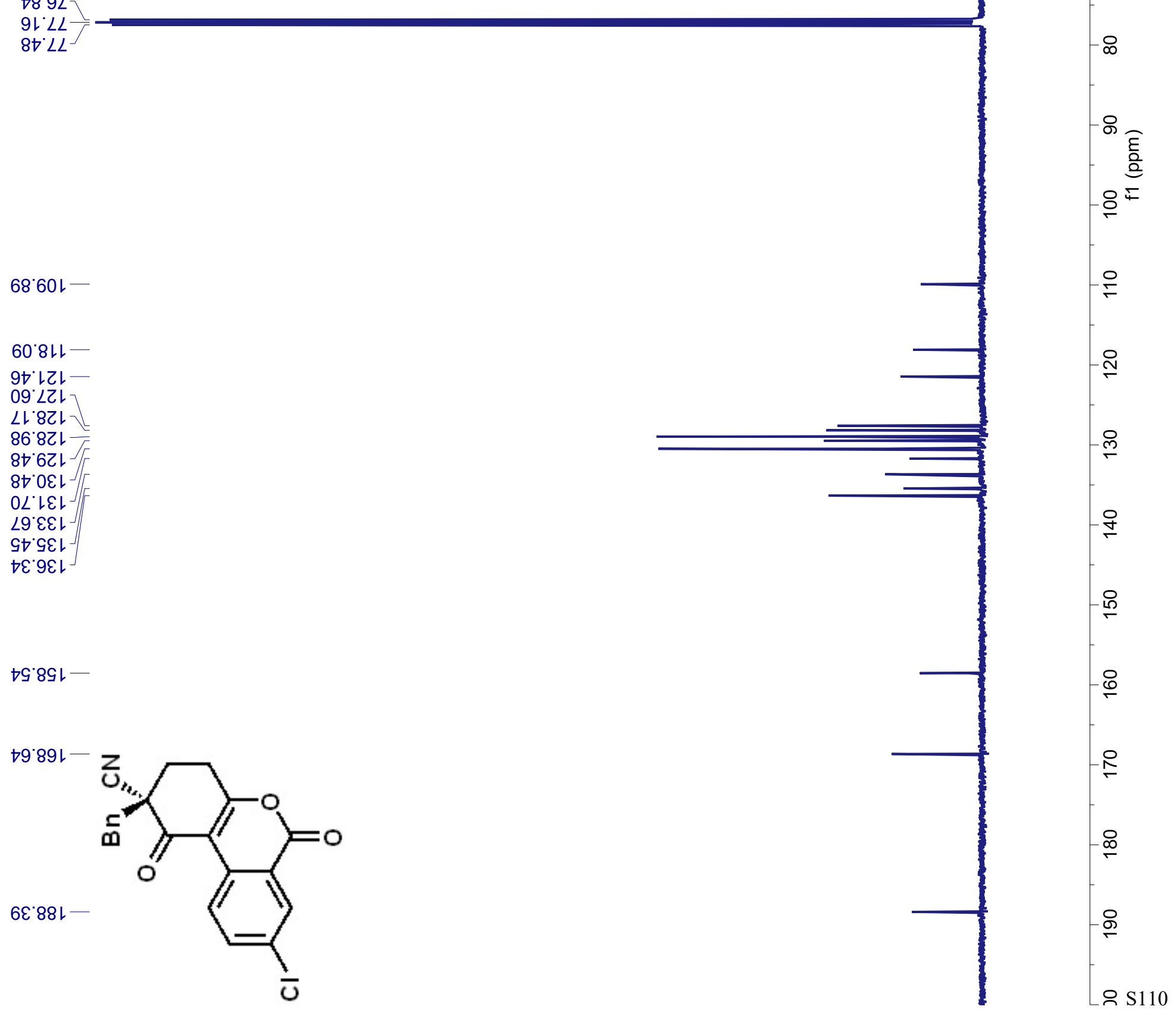


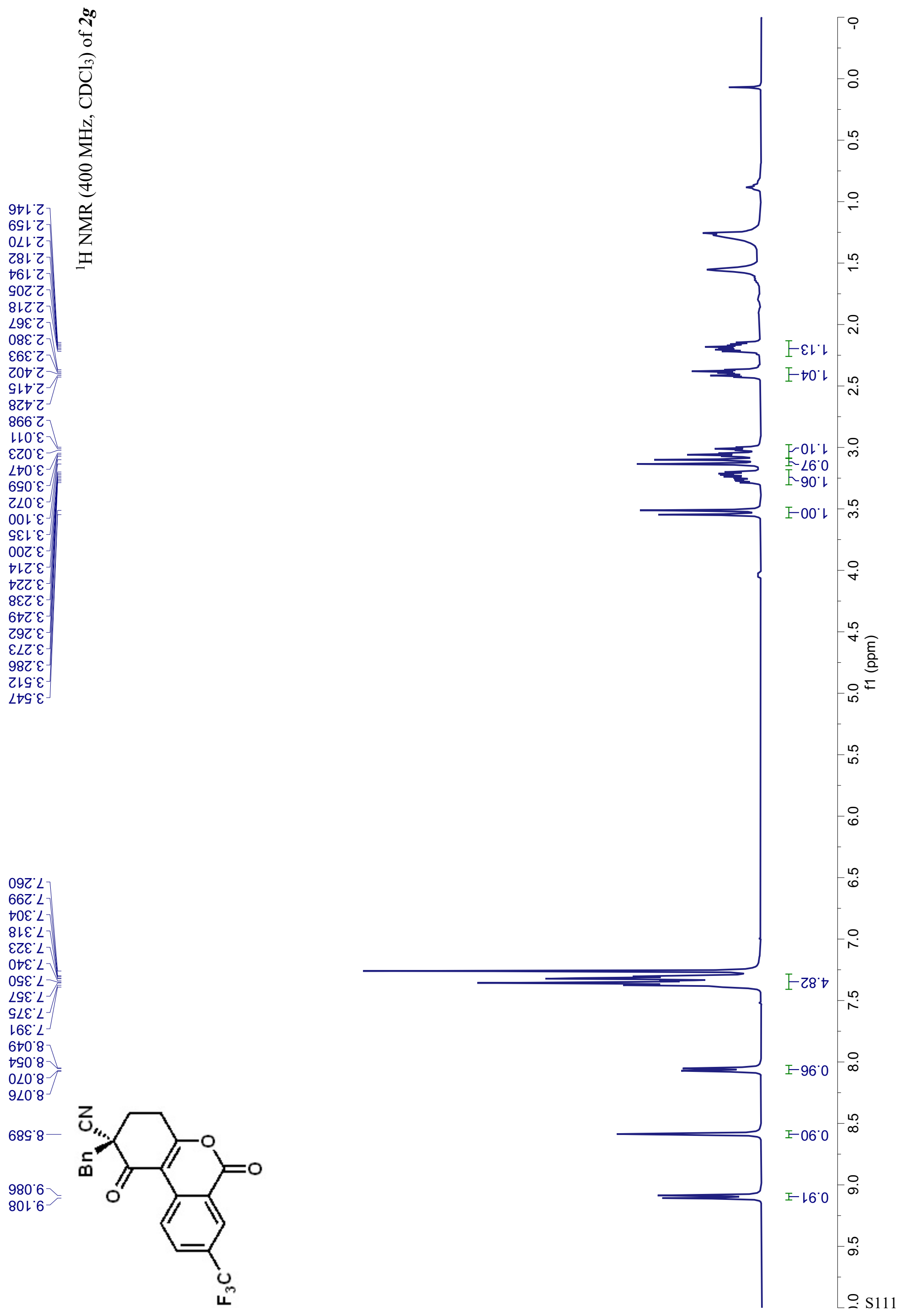



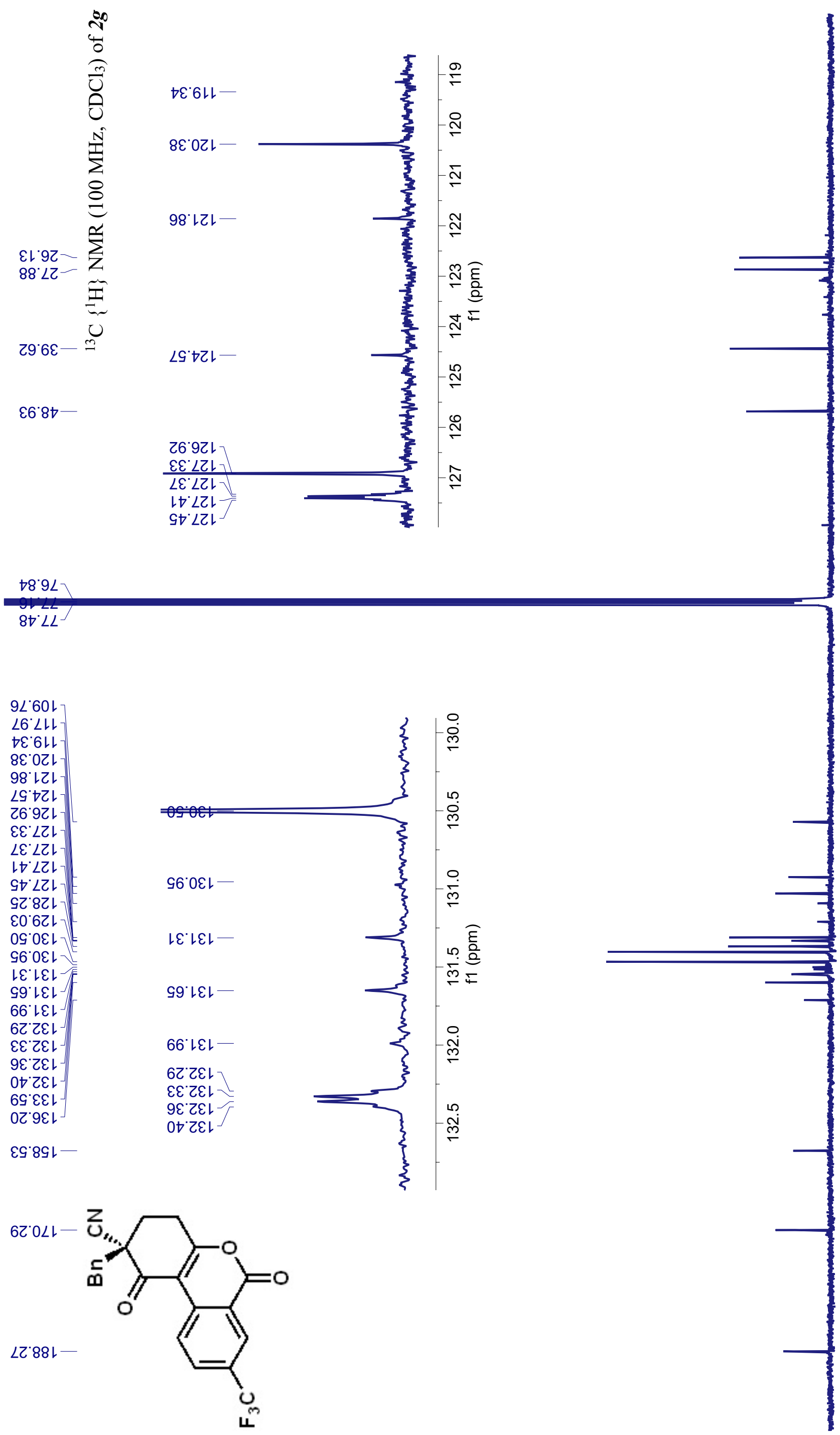


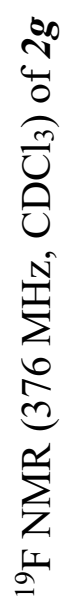

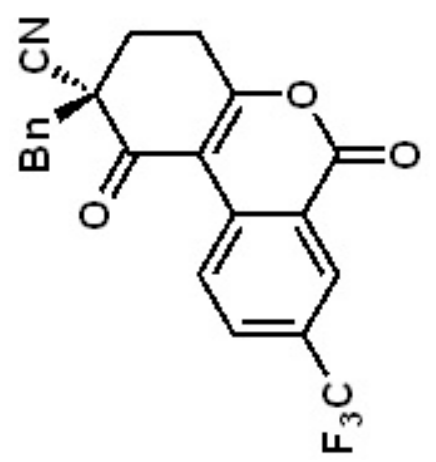



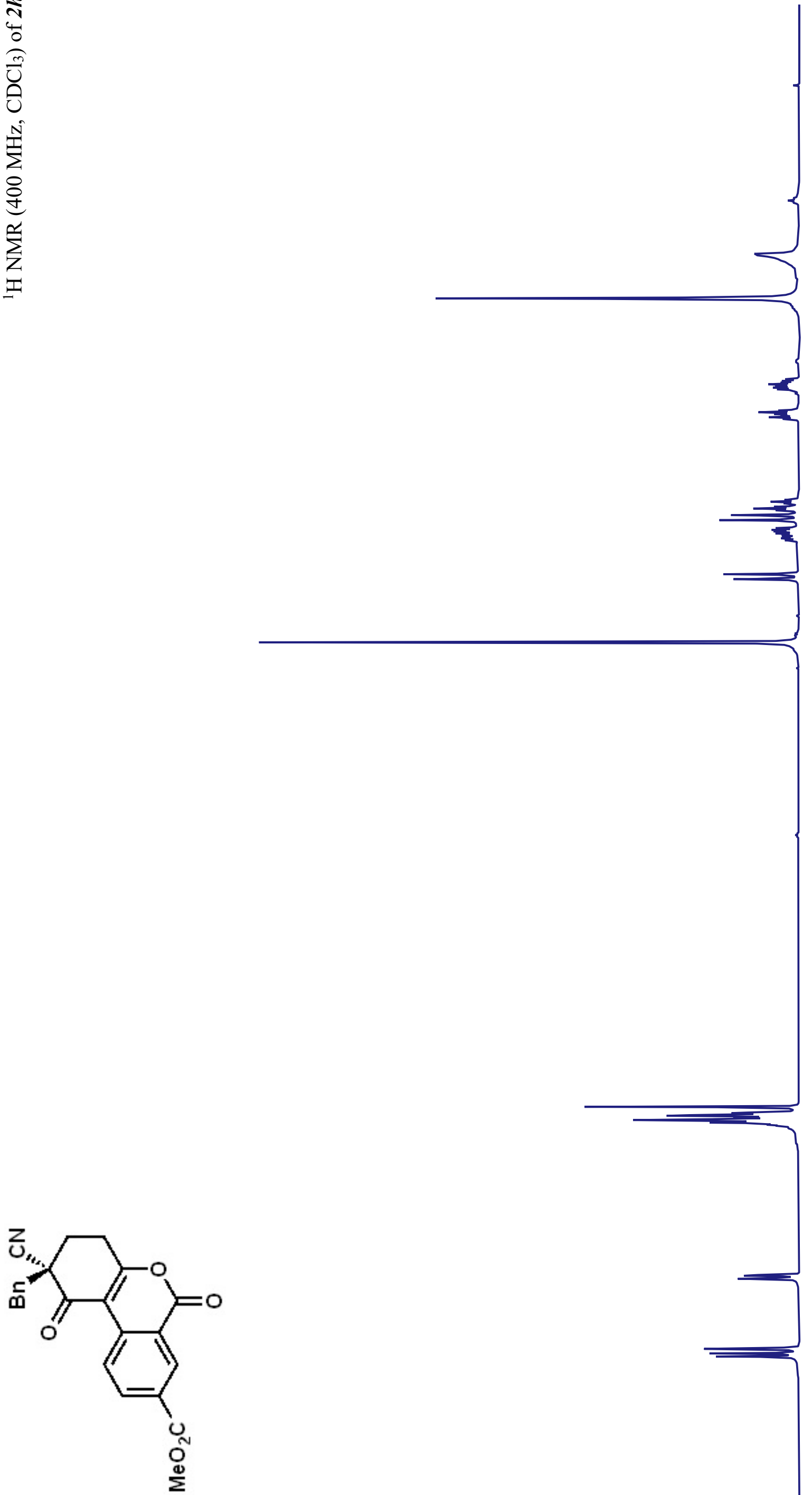


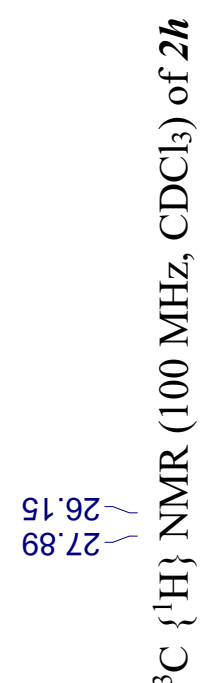

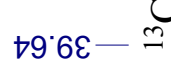

$96.87-$

$18.29-$

$\neg 8^{\circ} 9 L$

$80^{\circ} L L$

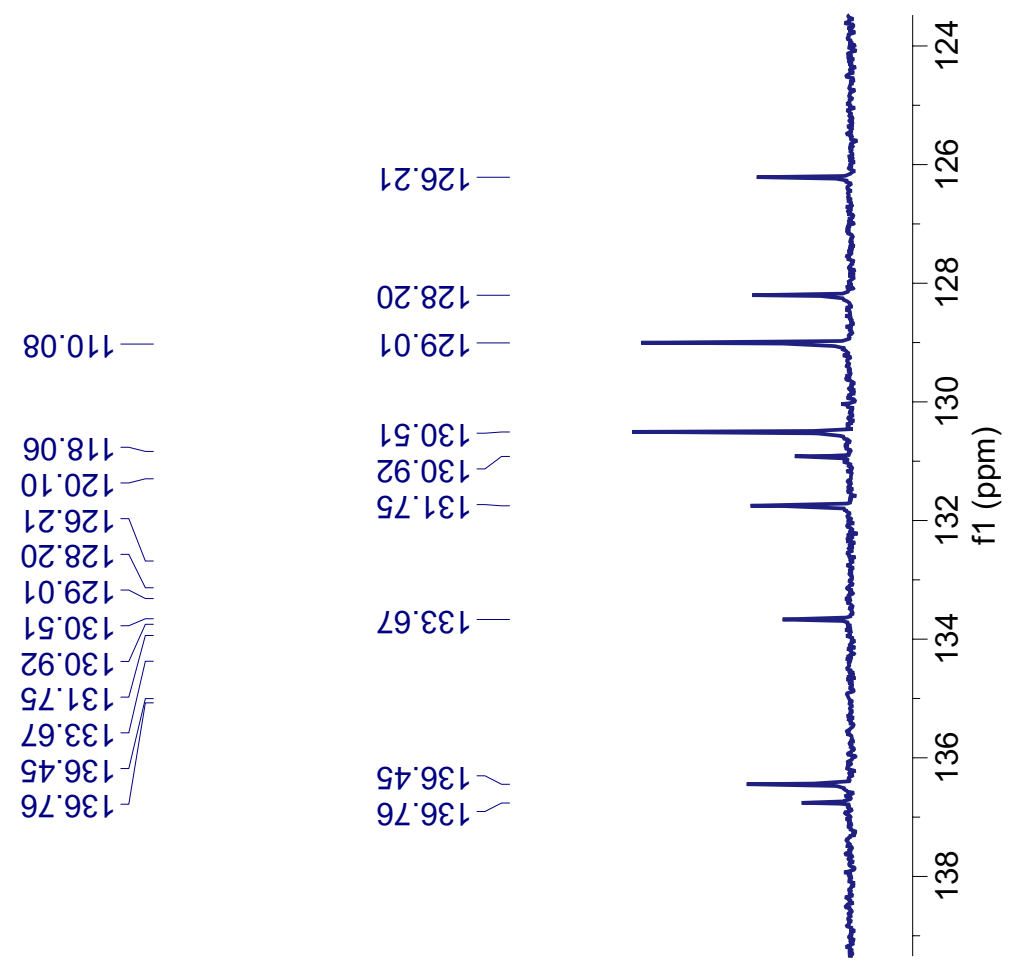

98.89レ-
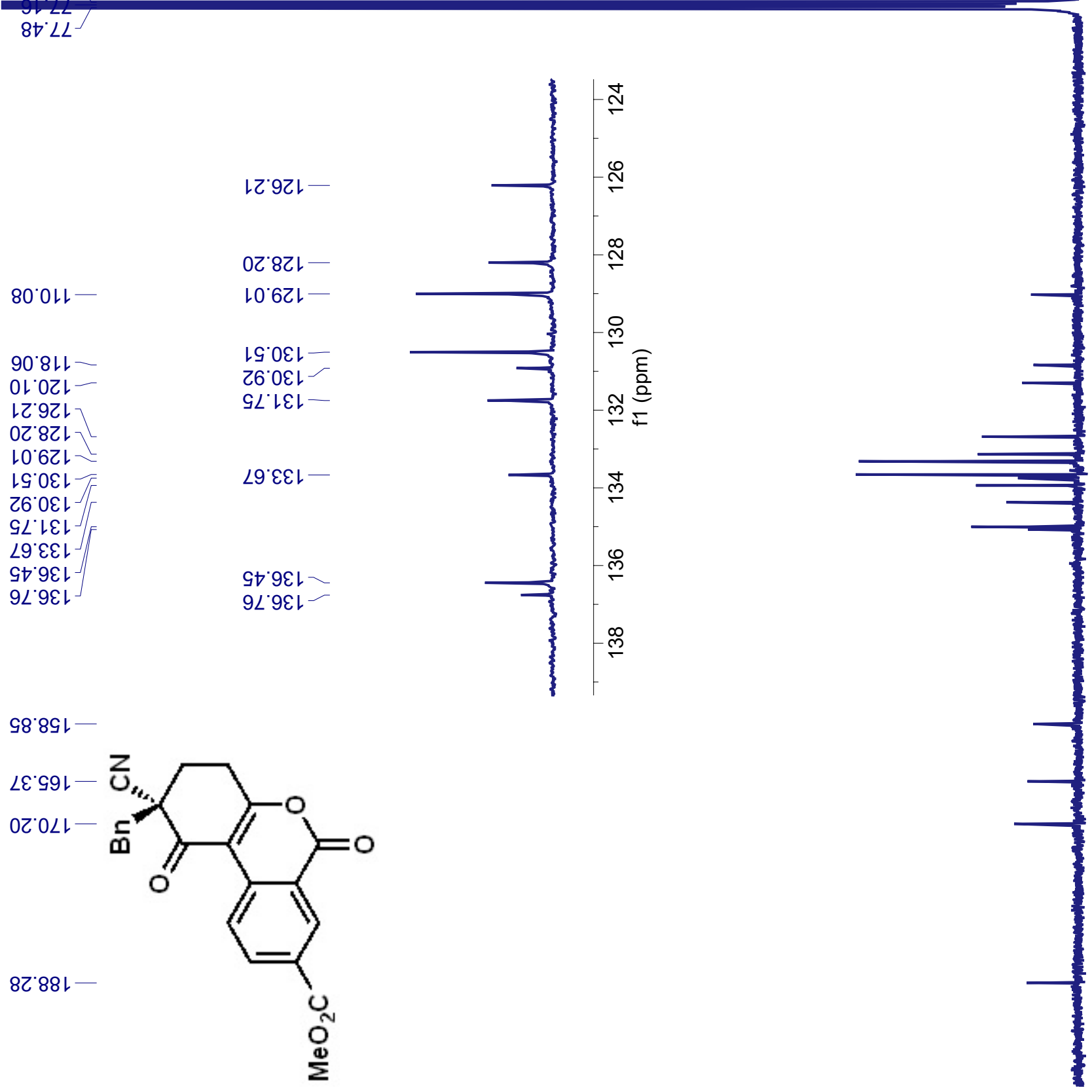

$\infty$

오

है
$8^{\frac{2}{2}}$

읃

온

$\stackrel{m}{\leftarrow}$

온

윰

8

$\frac{1}{2}$

움

욤 


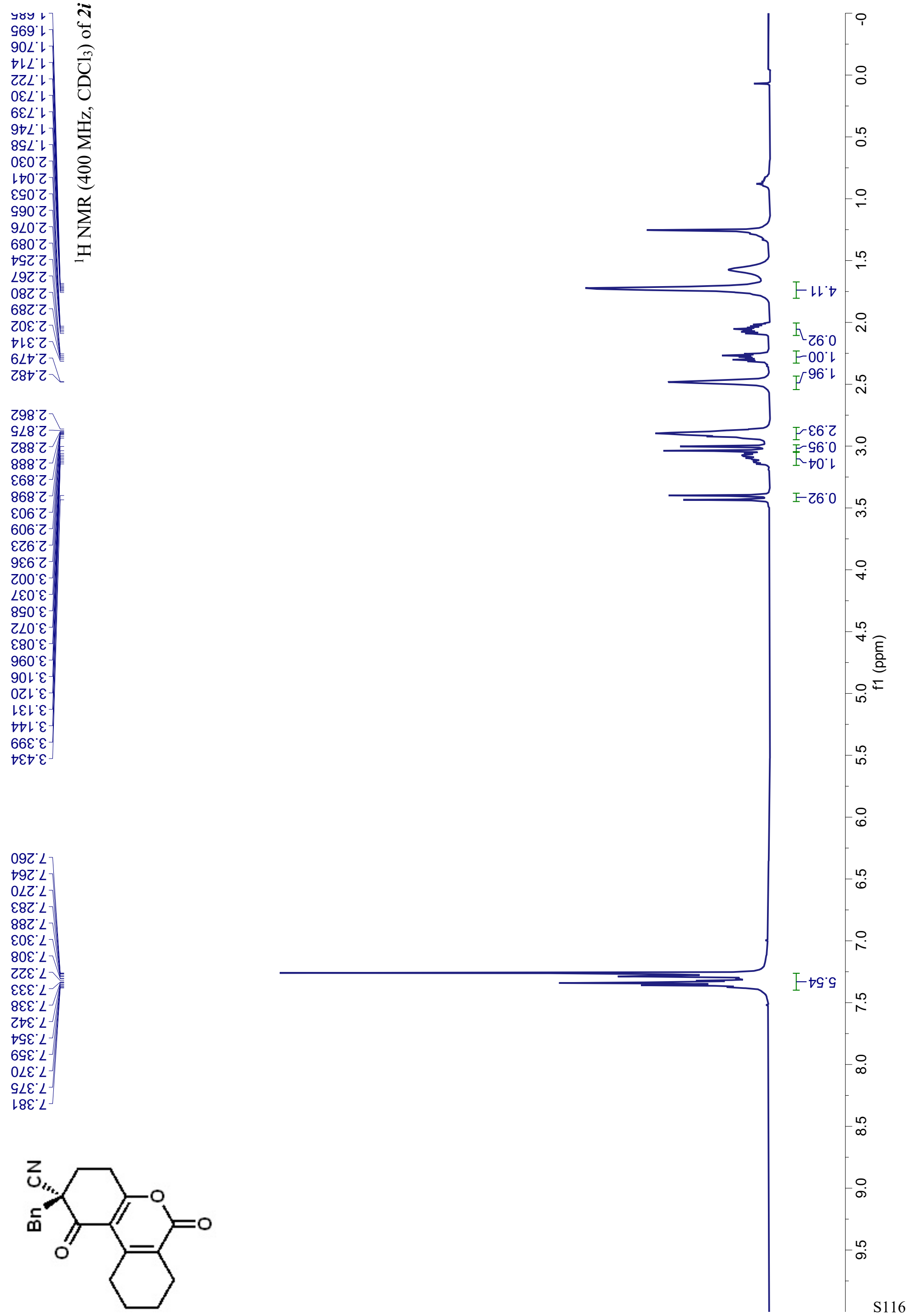



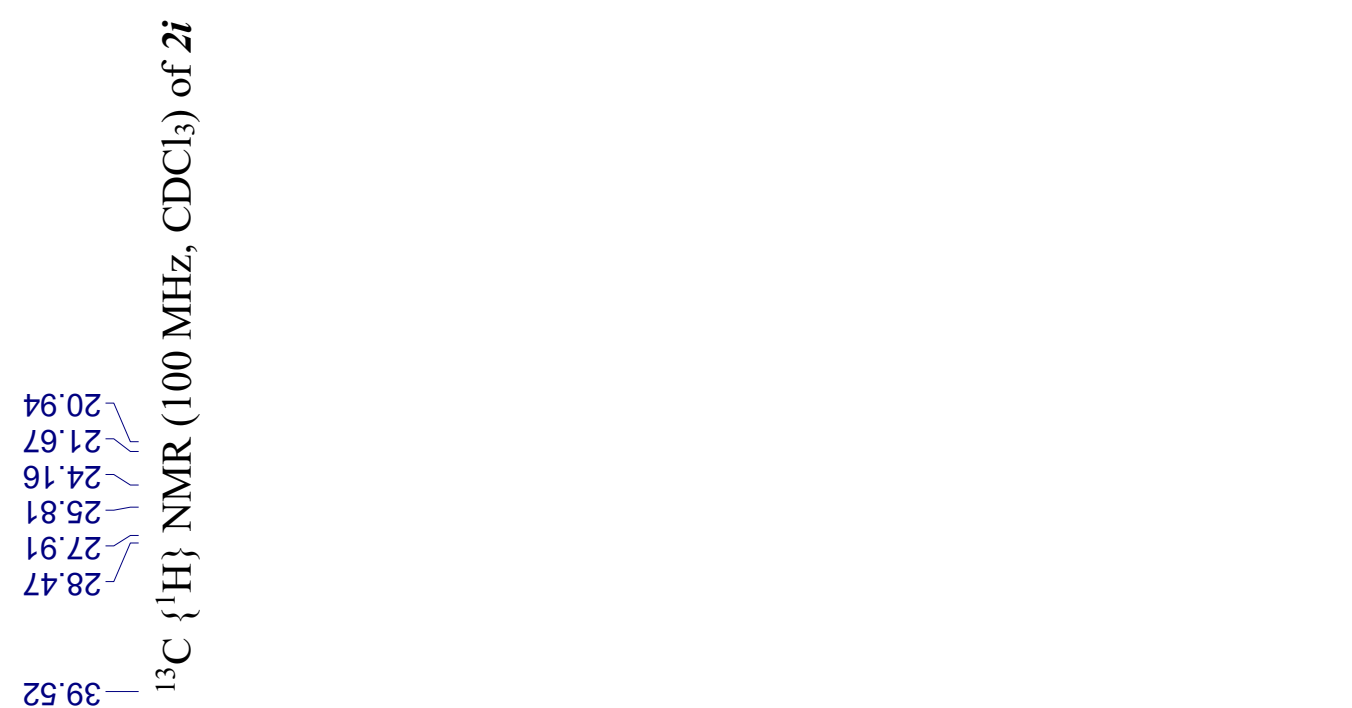

$76.87-$

$78: 9 L$

9L'LL

เย'ยレ

LC8L -

乙L乙Zレ

60.8ZL

Z6.8Z

S† $0 \varepsilon L$

乙8' ${ }^{\circ}$

†S.OSL -

†6.69 -

96.69L-

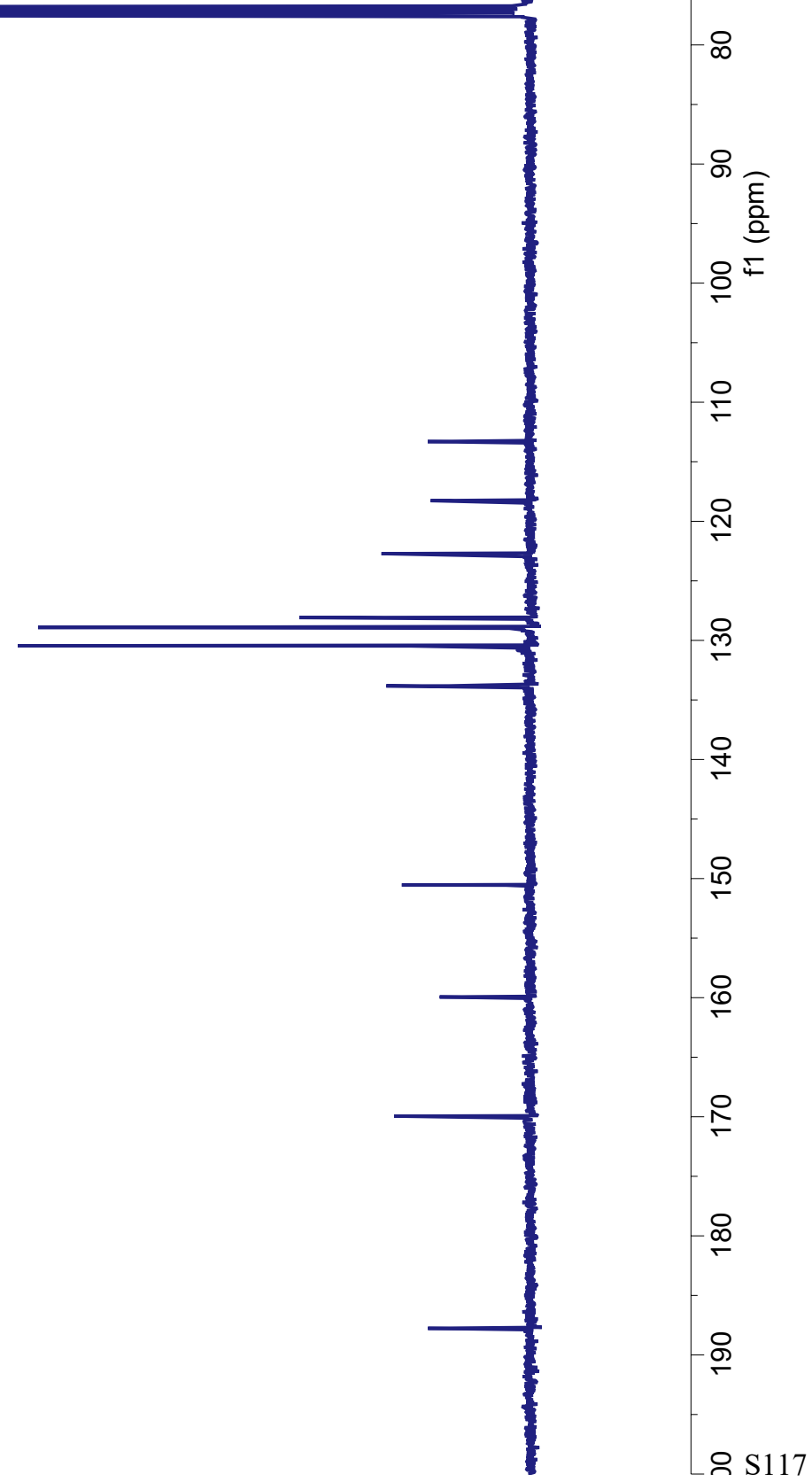




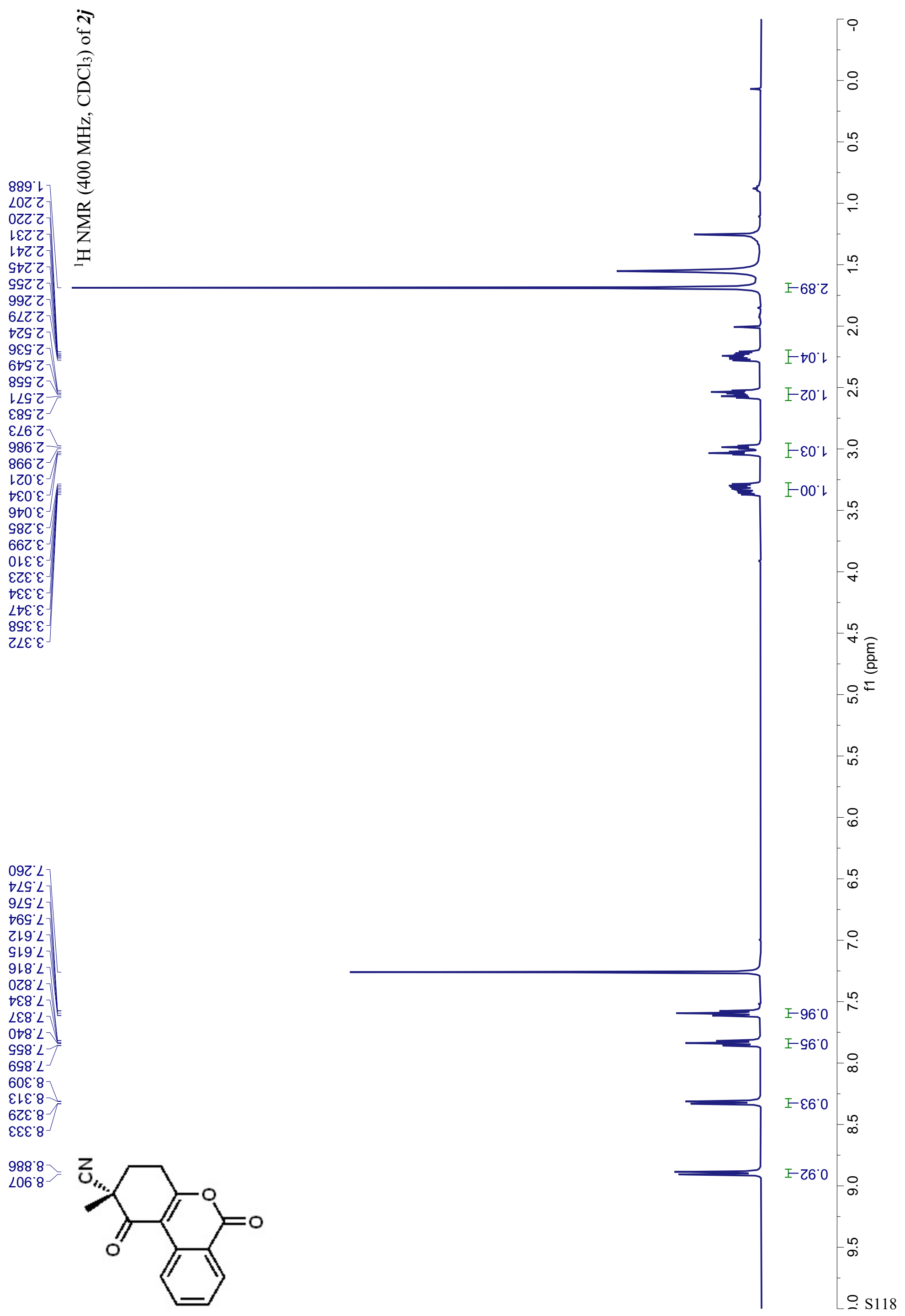




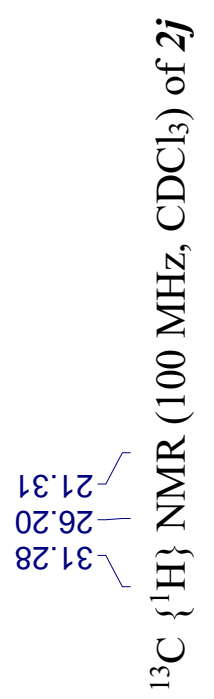

9เ๋๖ー

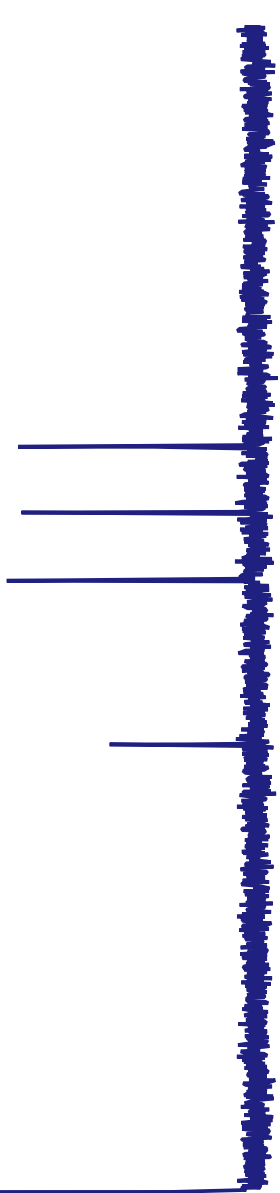

$78.9 L$

$87^{\circ} \angle L$

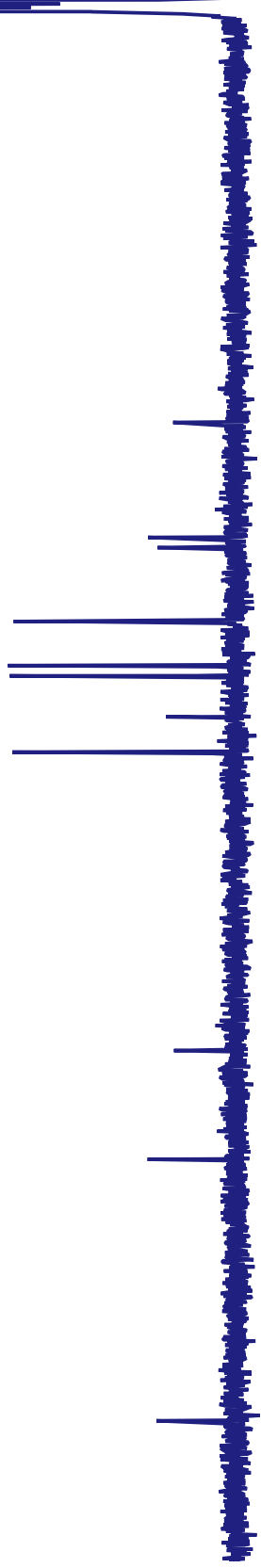

$\infty$

- 요

ルLOLー

6L6L

$00^{\circ} 021$

๕8'ตZL-

乙ย'6ZL

$\varepsilon L^{\circ} 0 \varepsilon \downarrow$

$\angle \mathcal{E} \varepsilon \varepsilon-$

Sเ'9el-

EL'6GL-

乙ย.89เ-

L6.88L-

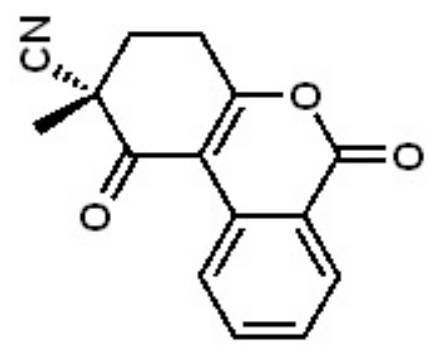




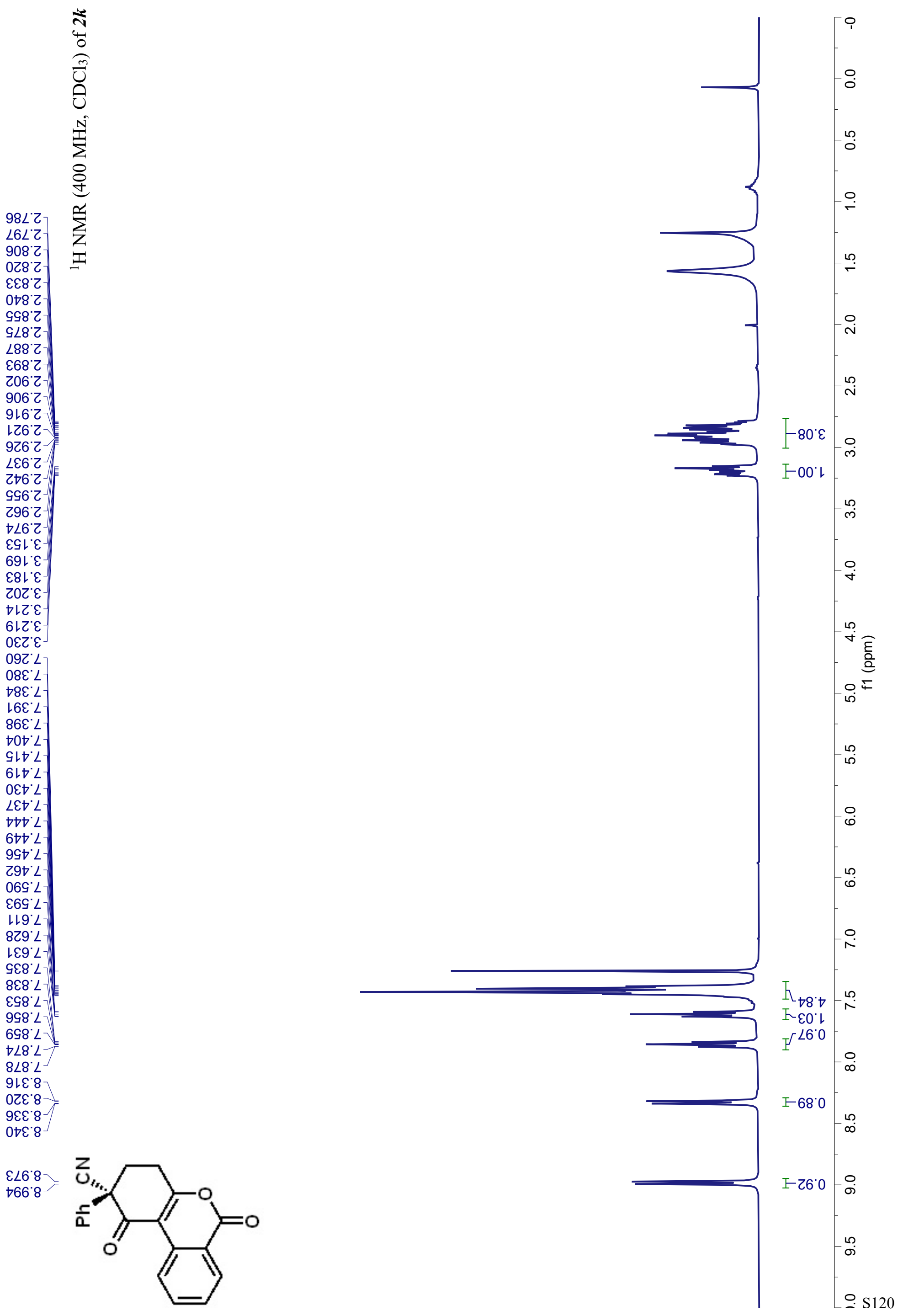




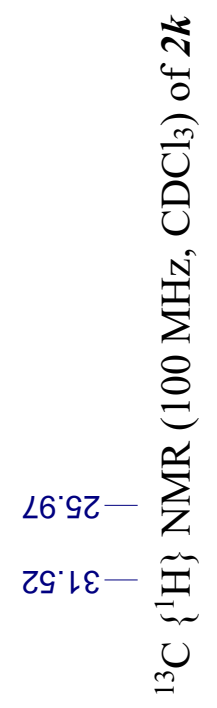

$78^{\circ} 9 L$

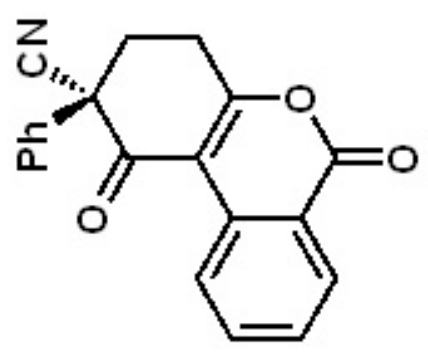




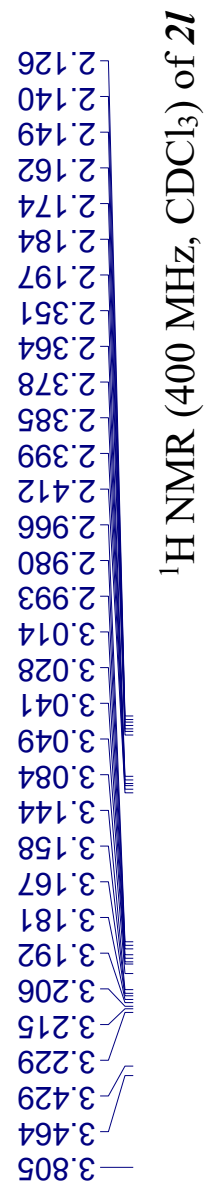

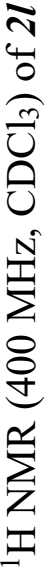

706.8 $766^{\circ} 8^{-}$
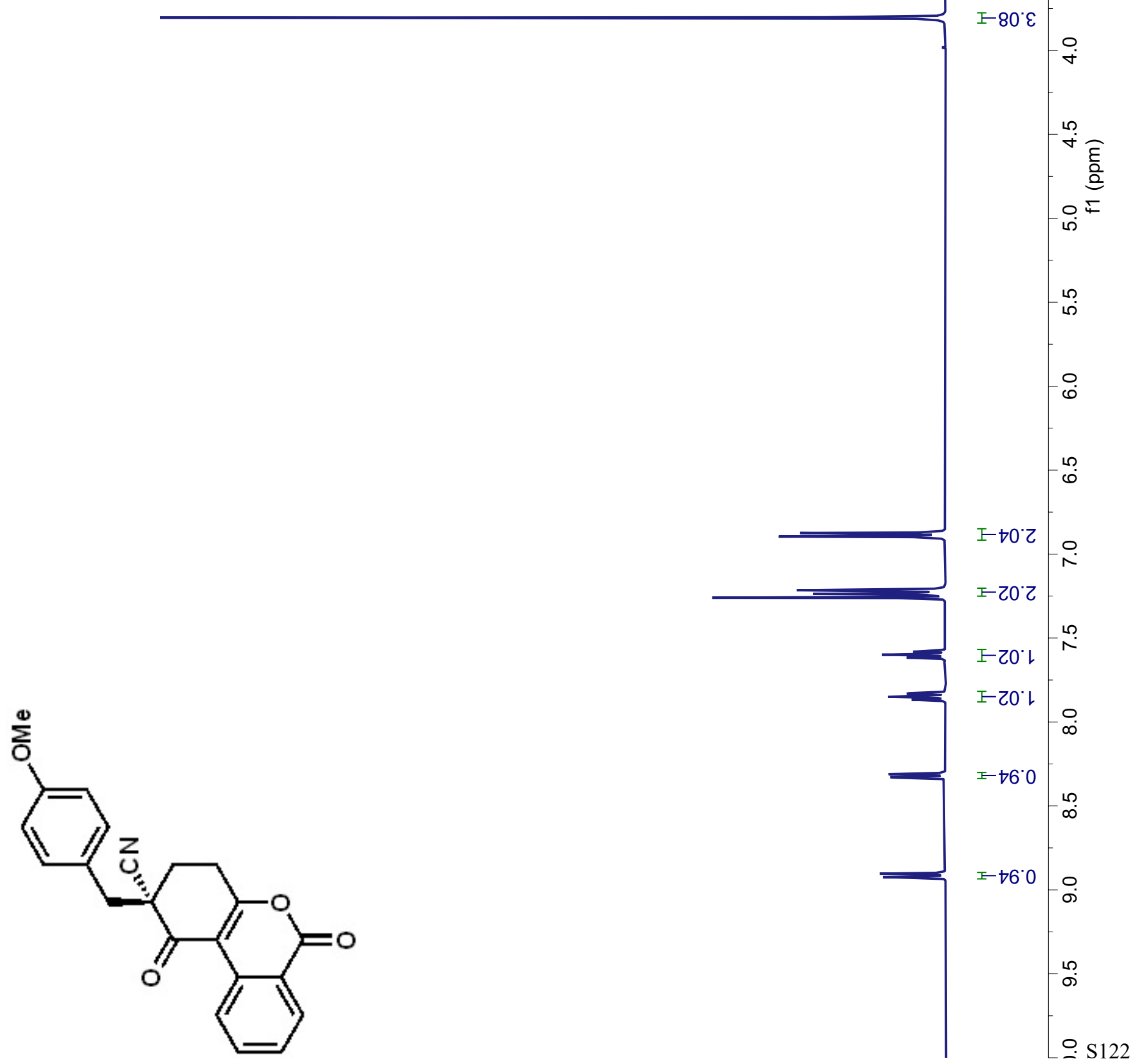


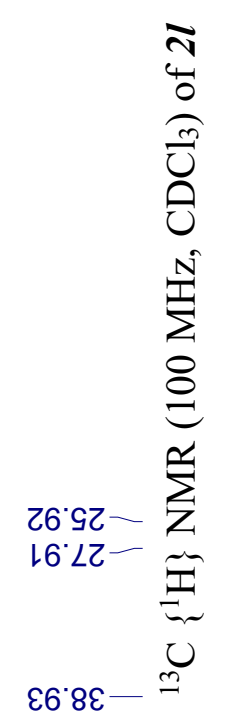

$0 \varepsilon^{\circ} 6 t-$

ع७ $99-$

$78^{\circ} 9\lfloor$

$87^{\circ} L L$

$9 \varepsilon$ ㅇレ

†ย・เル

ยจ8レ

ᄂOOZL

†9 G乙L

L8 G乙L

乙ะ.6乙L

†レ० $0 \varepsilon L$

9G'LEL

ธย' $\varepsilon \mathcal{~}$

LL $9 \varepsilon L$
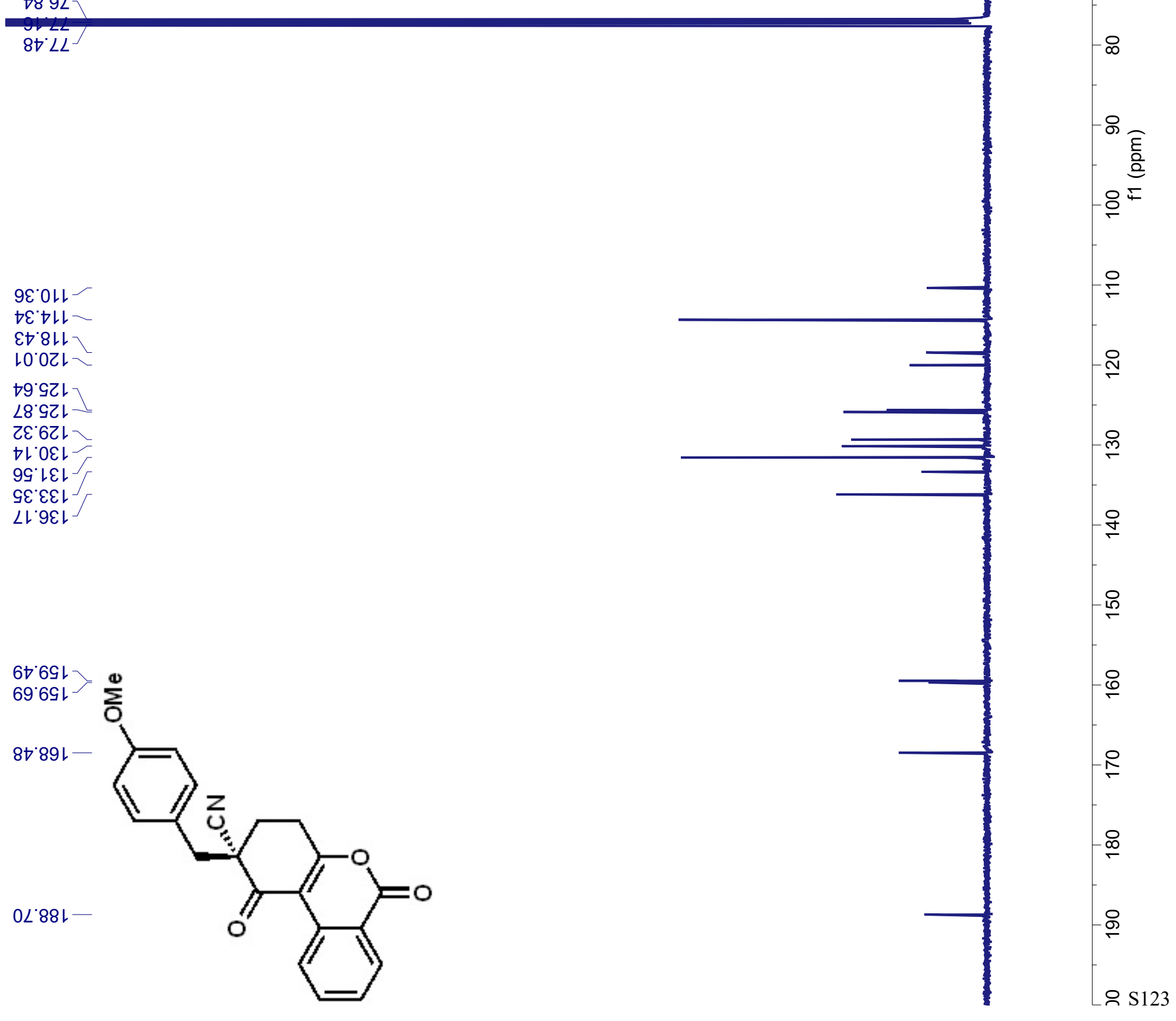


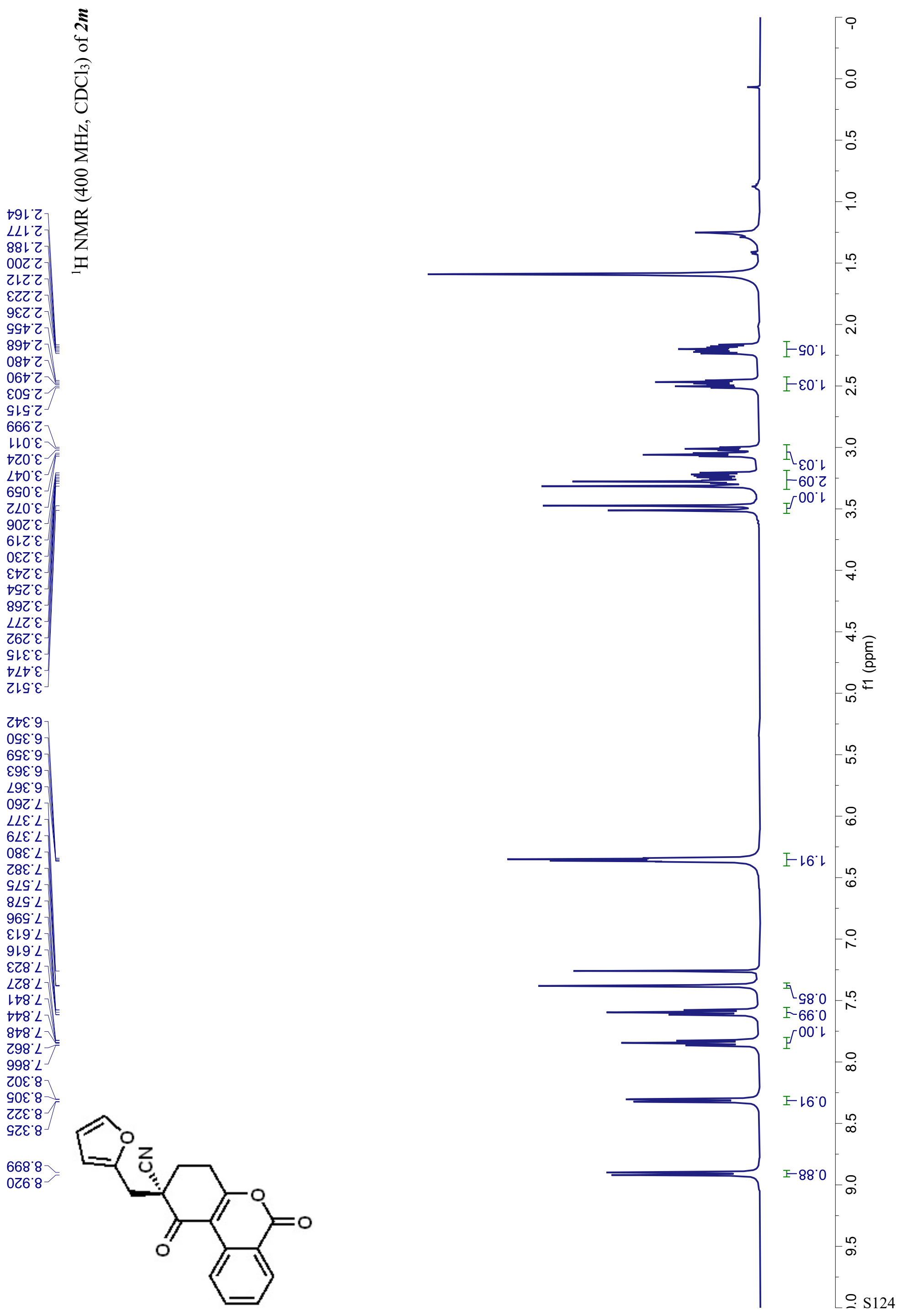




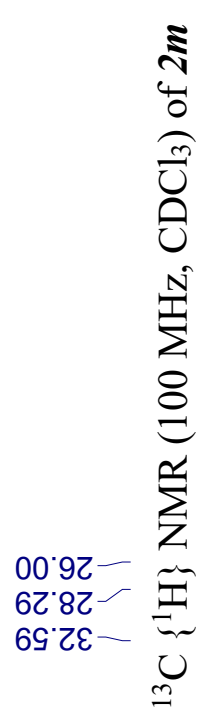

てち・8ヤー

$78^{\circ} 9 L$

8t $2 L$

Gレㅇㄴ

9200ルー

66 ㄴレ

$86^{\circ} 6$ L

S8.9ZL

† $6 Z$ -

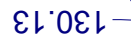

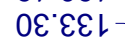

LL'9EL-

ย6ててレー

とカ・8ヤレー

ย9.69レ-

LL'89l-

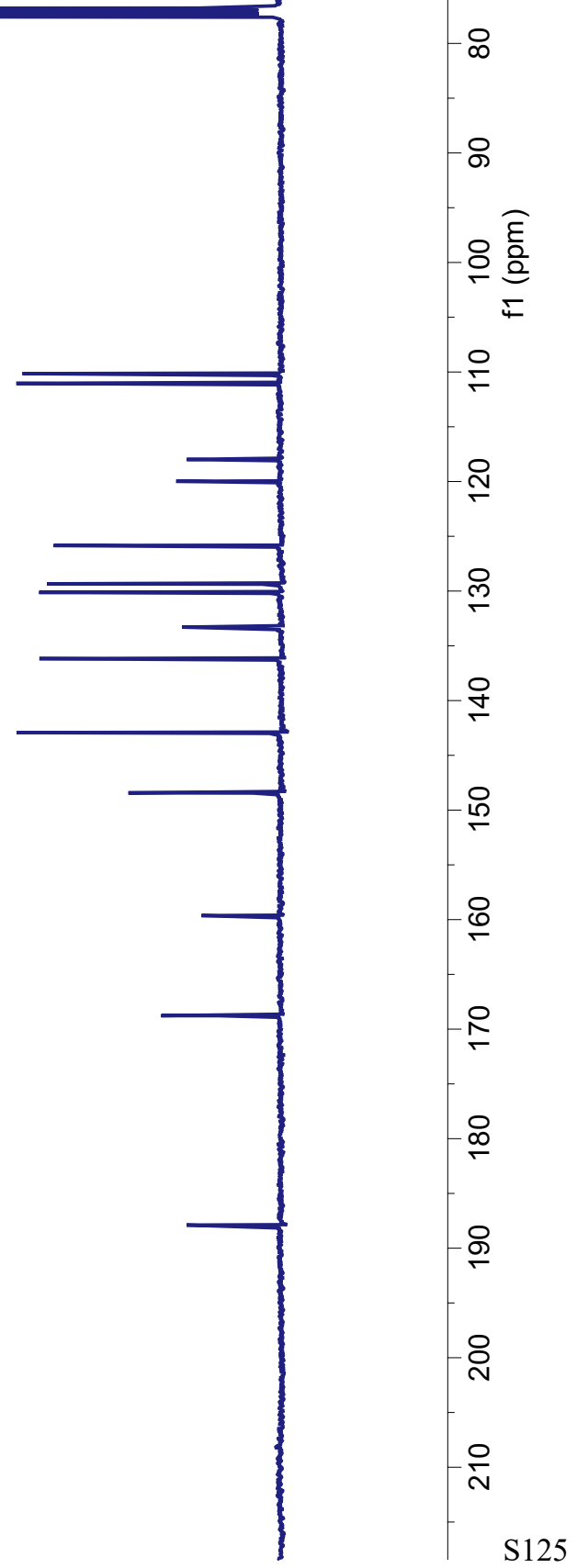

$68^{\circ} \angle 8 L$

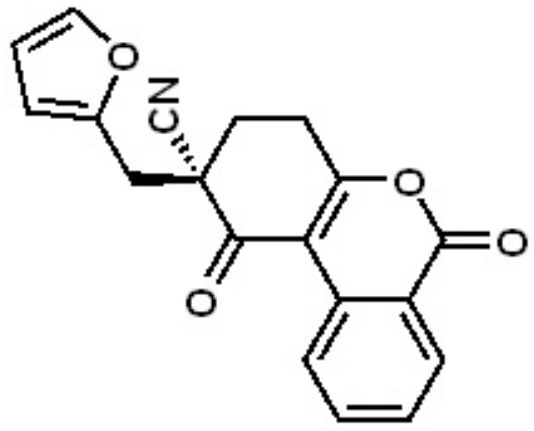




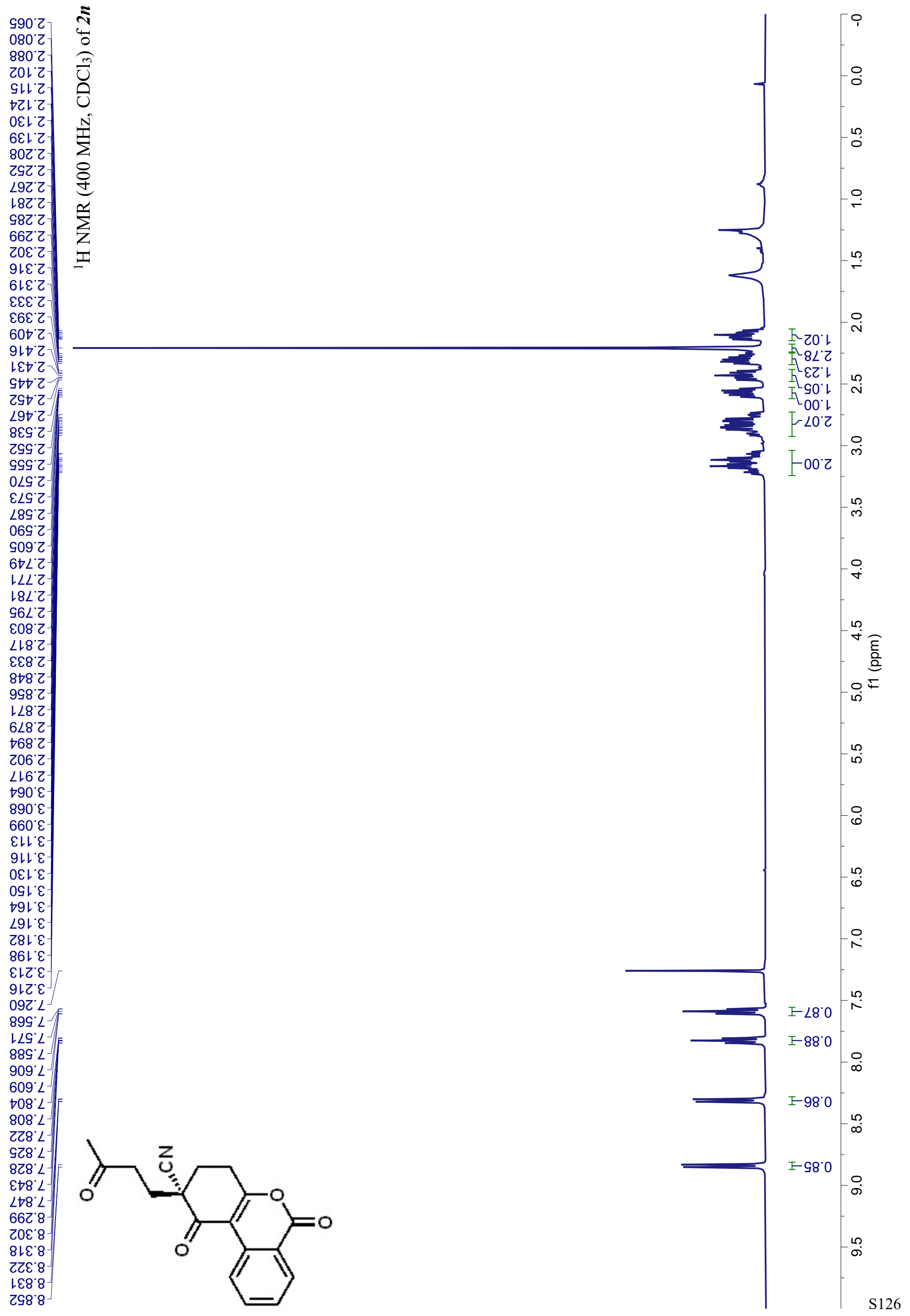




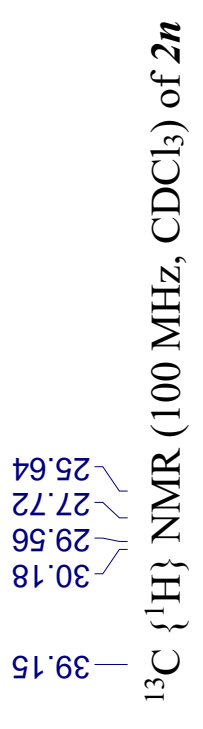

$21 \cdot 87-$

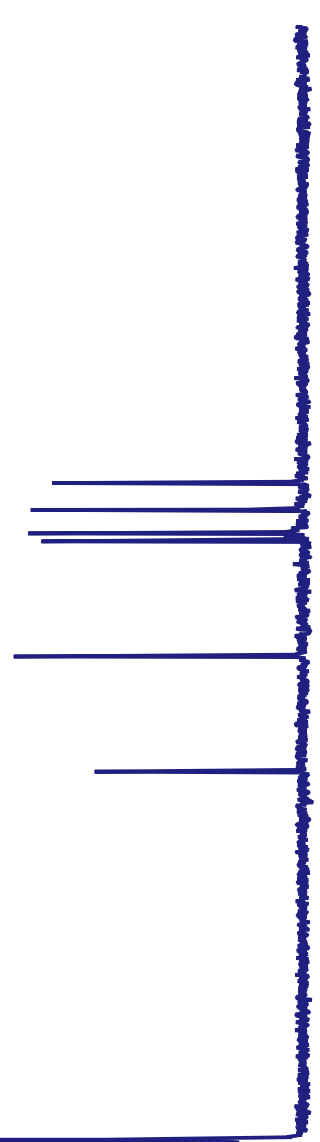

$78^{\circ} 9 L$

$87^{\circ} L L$

lOOLー

8ย'8レ -

00.02 L

LLGZL-

$\nabla \varepsilon^{\circ} 6 Z \downarrow$

GLOEL

$9 Z^{\circ} \varepsilon \varepsilon\llcorner$

จレ'9عᄂ-

ย9.69เ-

$26^{\circ} \angle 9 l-$
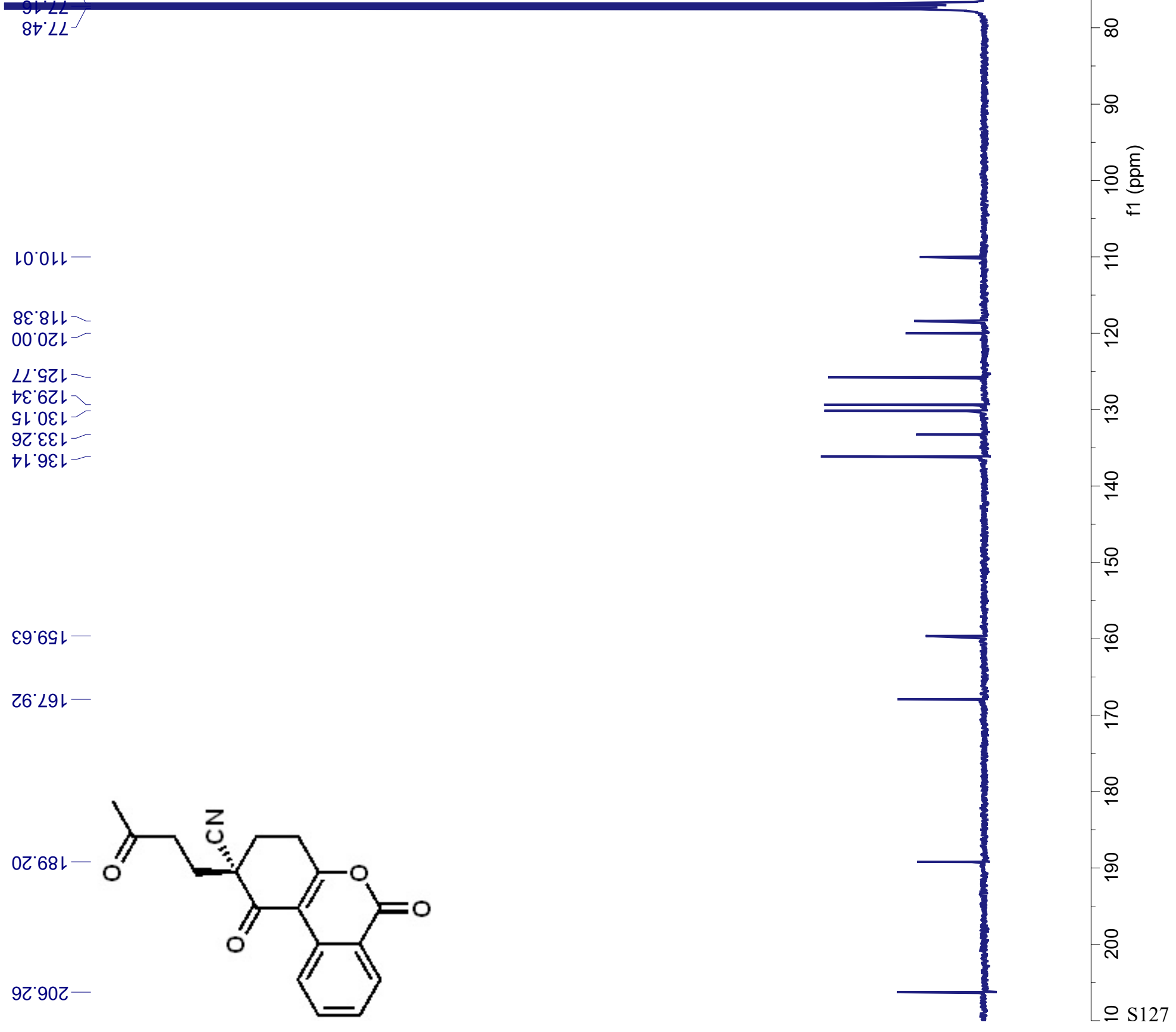


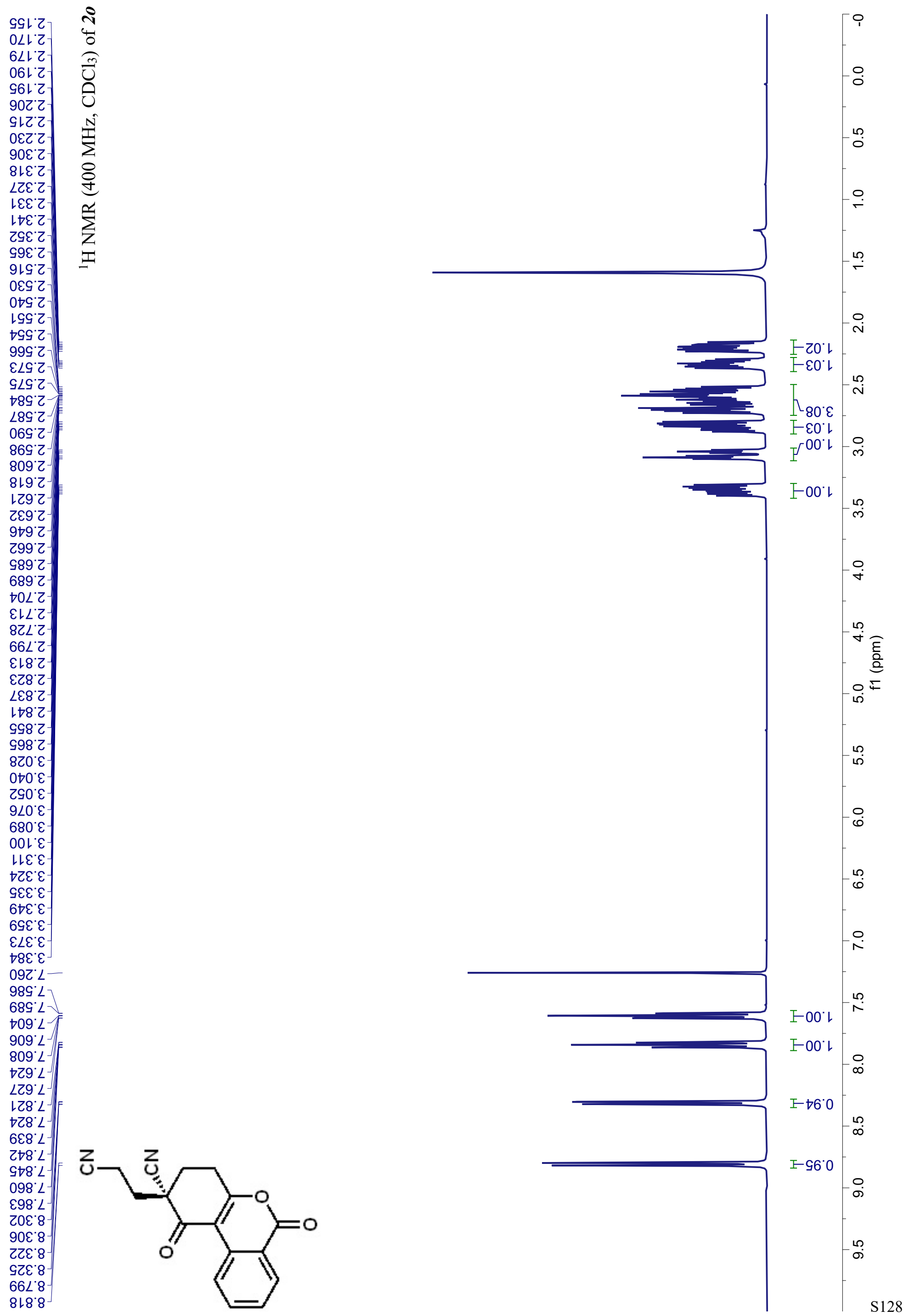




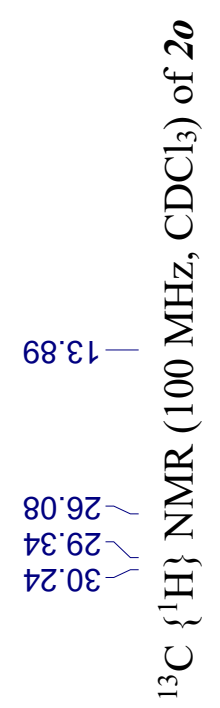

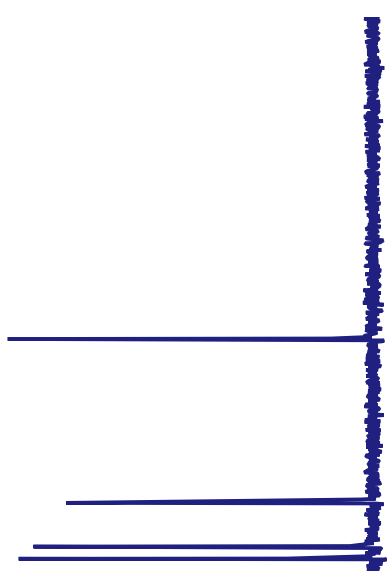

レレー

$78 \cdot 9 L$

乙20ルー

98.9レ

$928 \mathrm{~L}$

686 L

Z9.9Z। -

89.6ZL

Gट $0 \varepsilon L$

$88^{\circ}$ 乙E

$\angle Z^{\prime} 9 \varepsilon L-$

ยE'6Sレ-

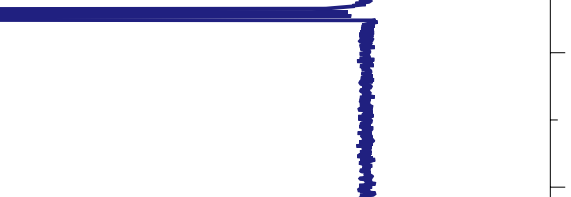

$\infty$

흘
을 $^{-}$

읕

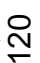

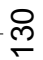

움

$\stackrel{6}{\circ}$

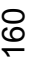

2t $891-$

t9 $281-$

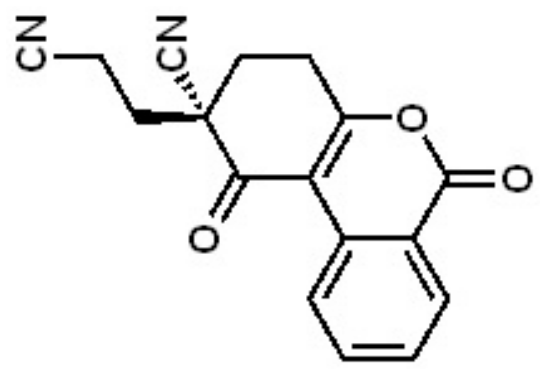



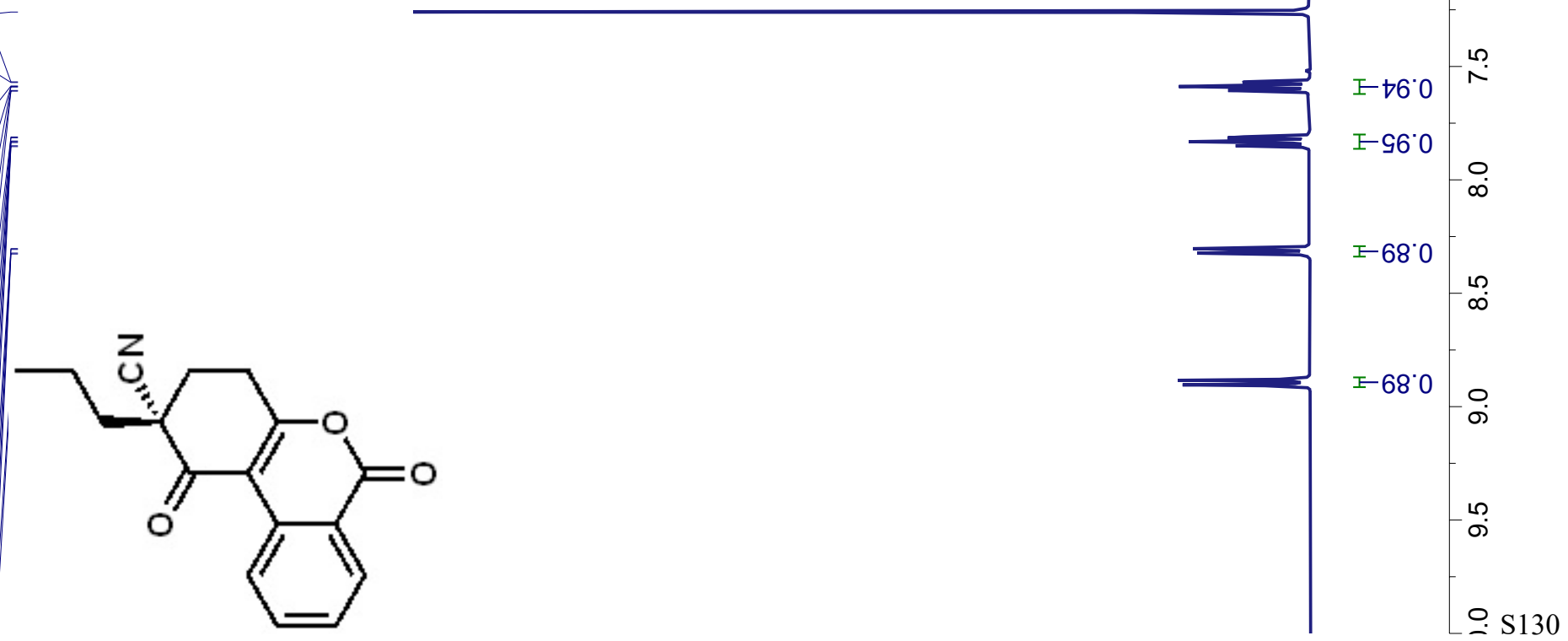


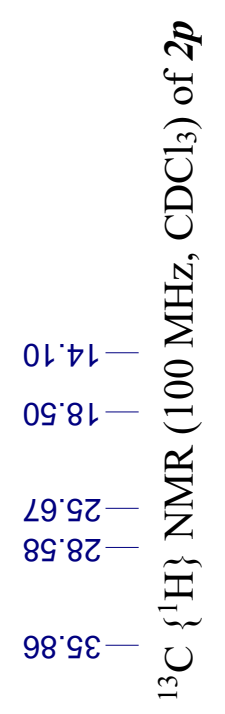

$\angle L \cdot 87-$

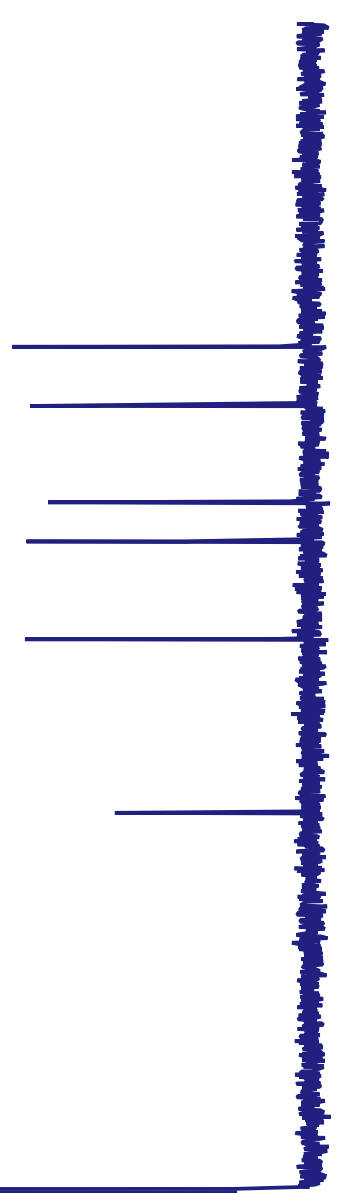

$78^{\circ} 9 L$

$8 \nabla^{\circ} L L$

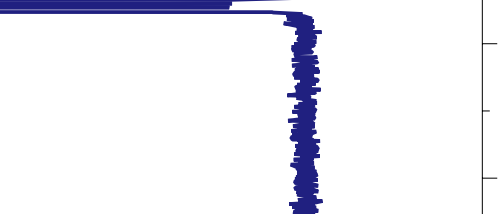

เo.0เ上-

6L8ルー

G० 02 L

L8.9ZL

$8 \mathrm{Z}^{\circ} 6 \mathrm{ZL}$

ᄂ $0 \varepsilon L$

$6 \varepsilon^{\prime} \varepsilon \varepsilon\llcorner-$

9l'9ع -

8L69レ-

S8 $\angle 91-$

乙768レ-

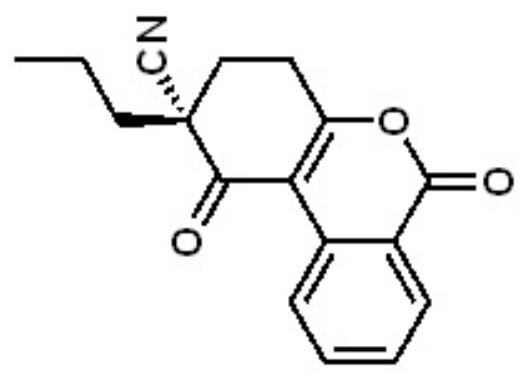




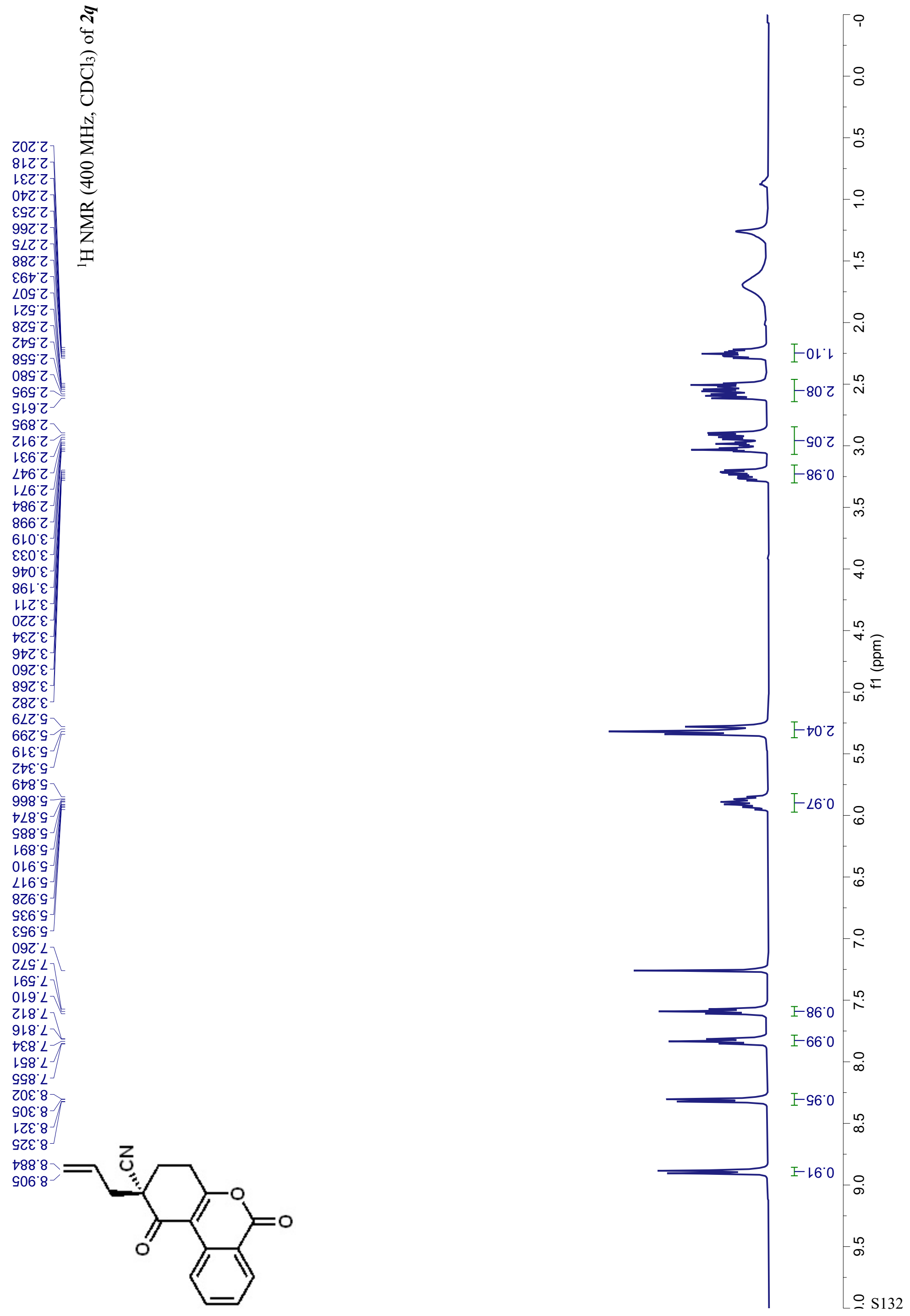



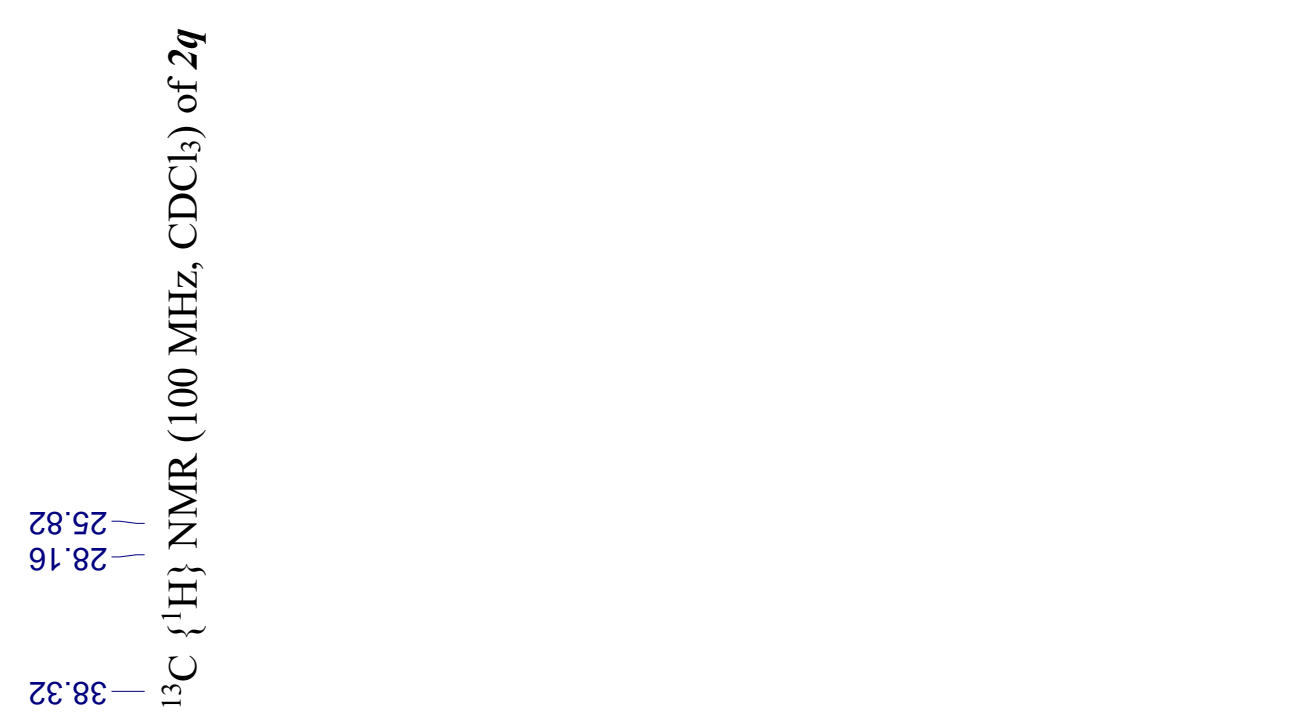

$6 L \angle b-$

$78^{\circ} 9 L$

8L8L

$00.02 L$

乙LレてL-

98.GZL

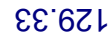

$\varepsilon L^{\circ} 0 \varepsilon L$

69.0ع

ᄂ $\varepsilon \varepsilon\llcorner$

LL $9 \varepsilon L$

69.69レ-

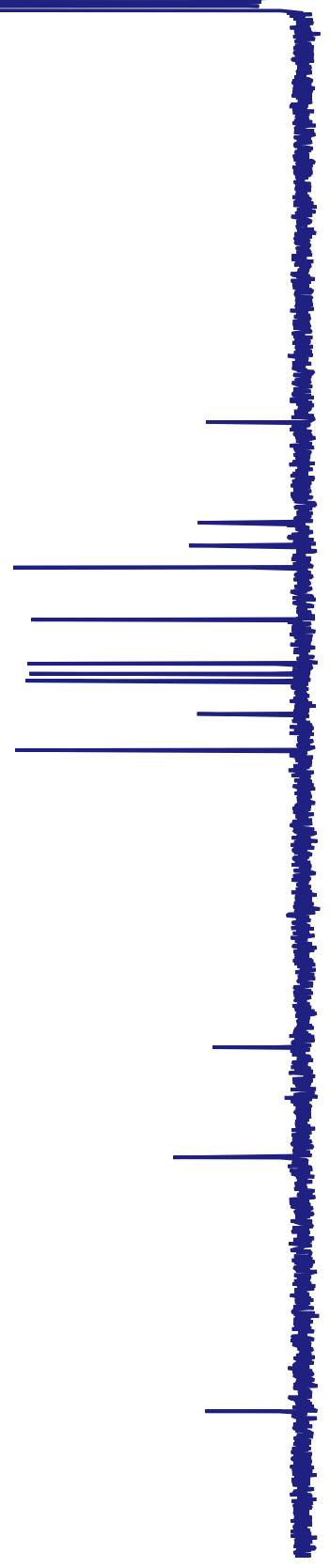

$\infty$

요

흥

용

읃

은

욤

암

온

$\stackrel{8}{\circ}$

เท8 89 -

67.881-

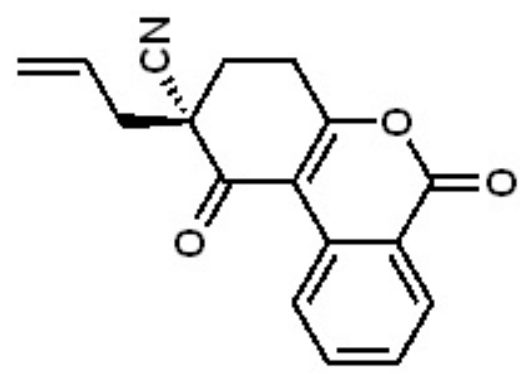




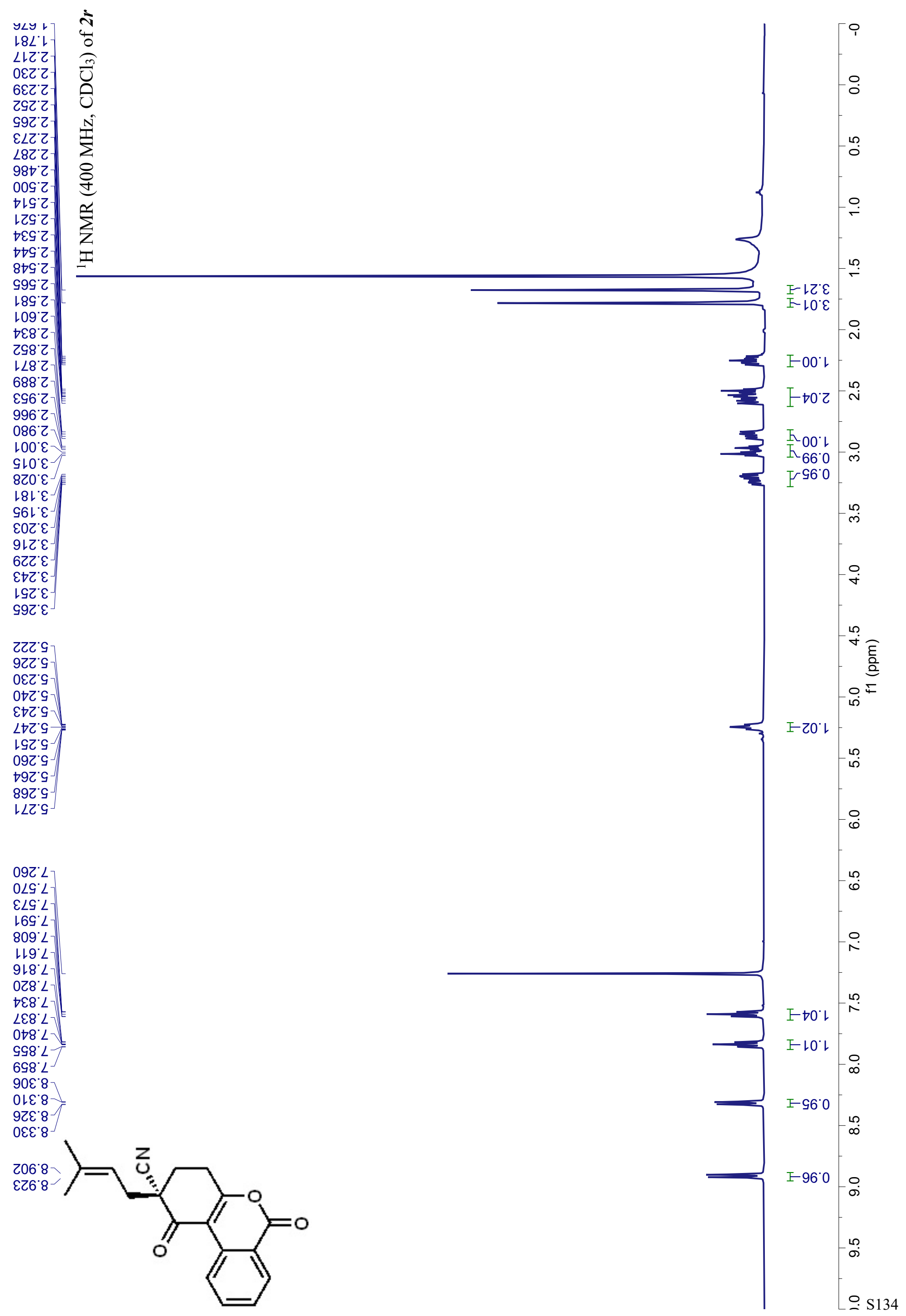



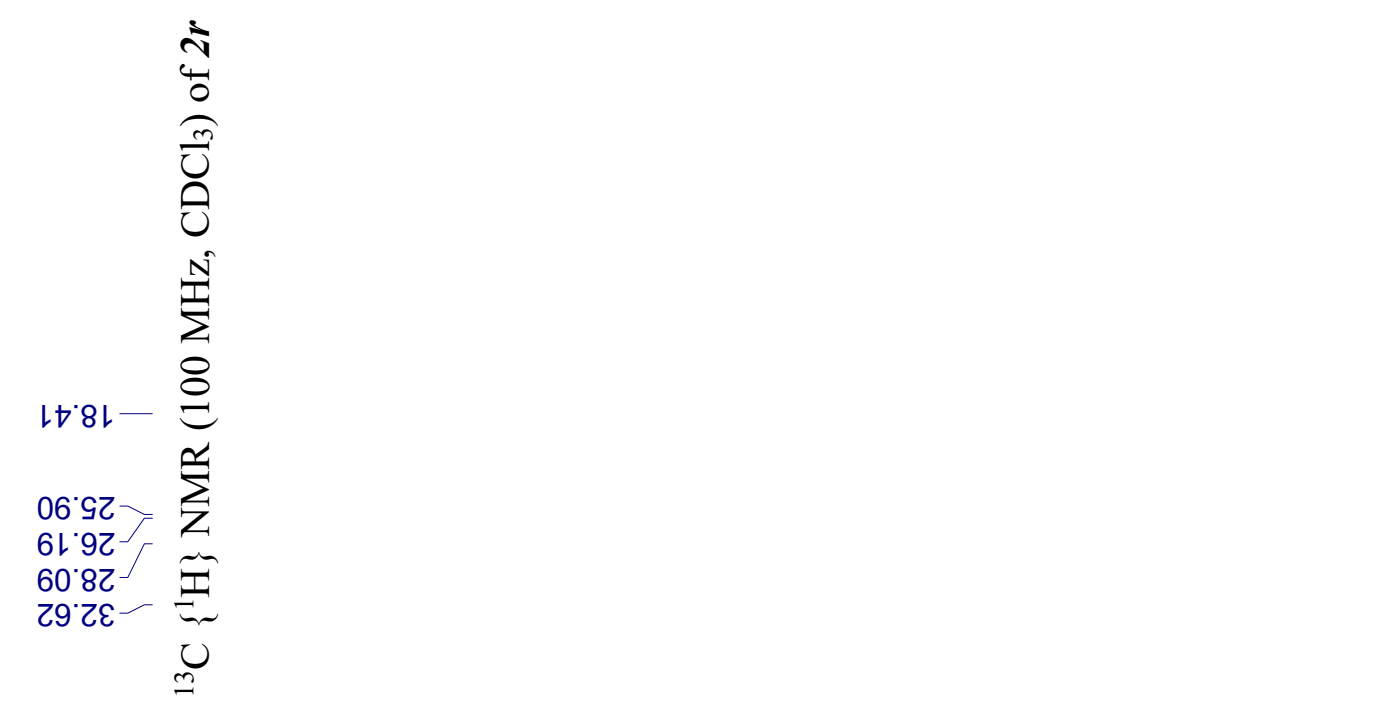

$09^{\circ} 8 \mathrm{~b}$

$78^{\circ} 9 L$

$87^{\circ} L L^{\prime}$

LZOUL

ยて9ルレ

$798 \mathrm{LL}$

10.0ZL

689てレ -

$8 \mathrm{Z}^{\circ} 6 \mathrm{ZL}$

LᄂOEL

乙† EEL -

GL'9عᄂ

69.8EL-

8L6GL-

$\succsim \varepsilon^{\circ} 89$ -

66.88 -
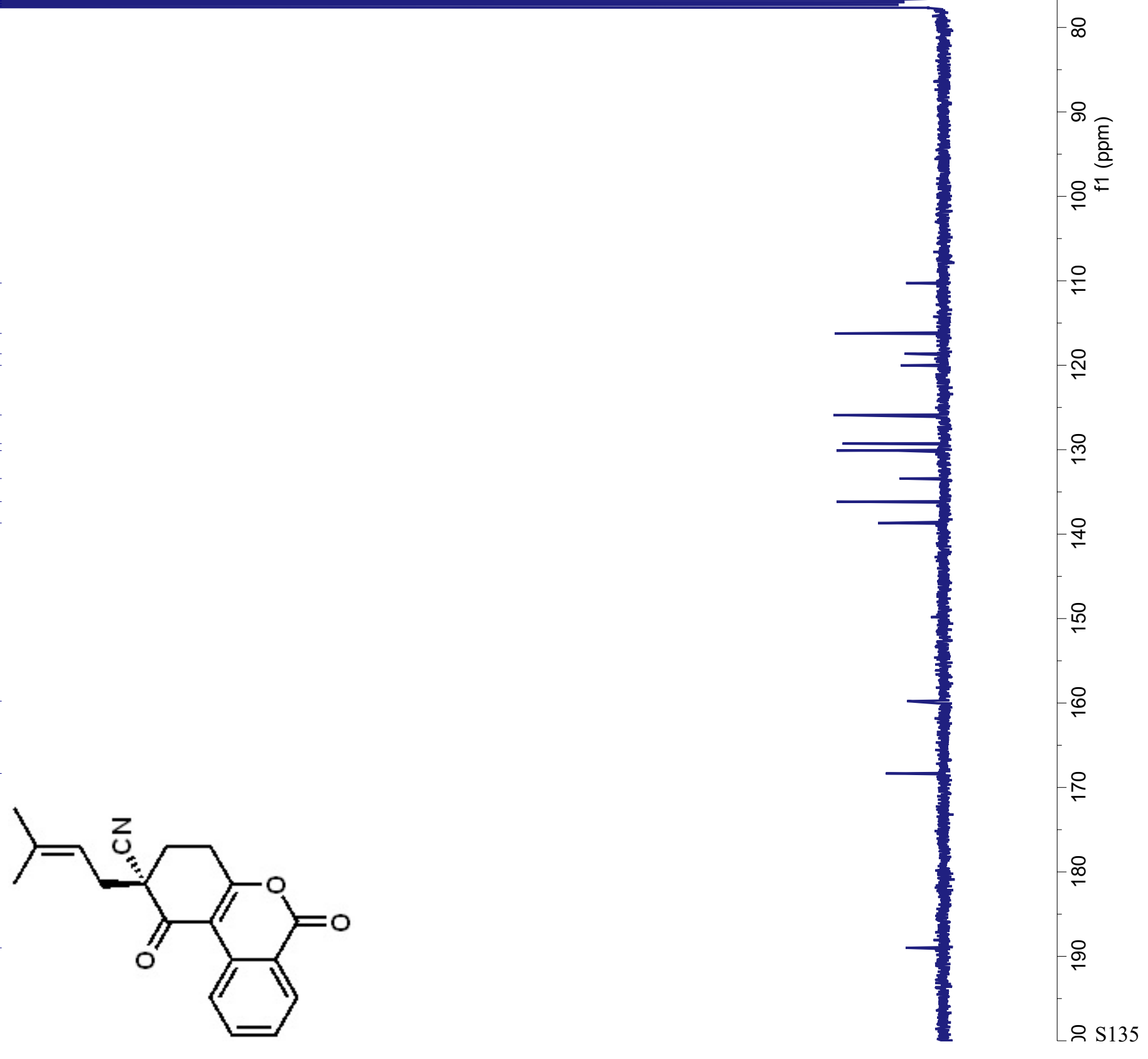


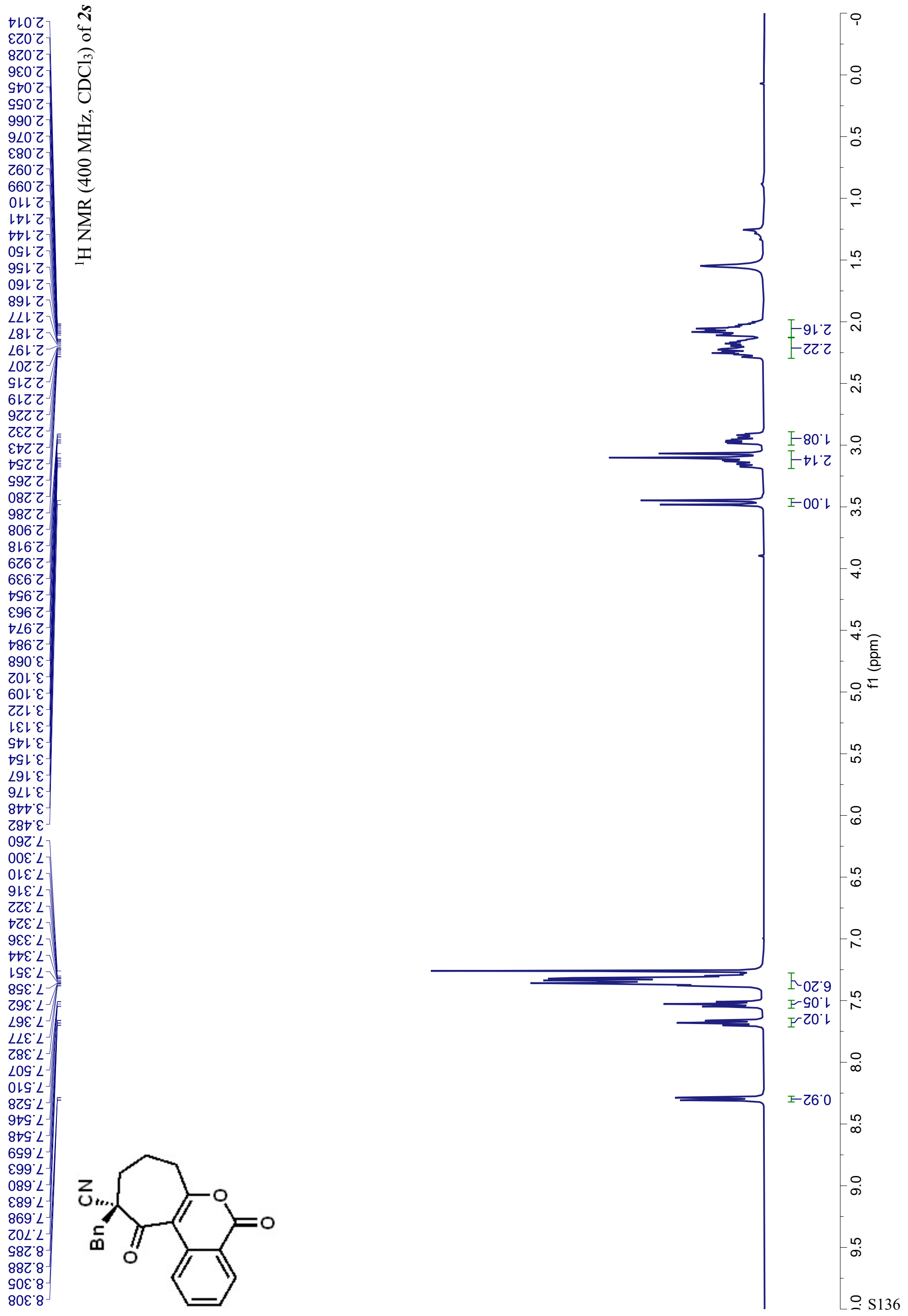



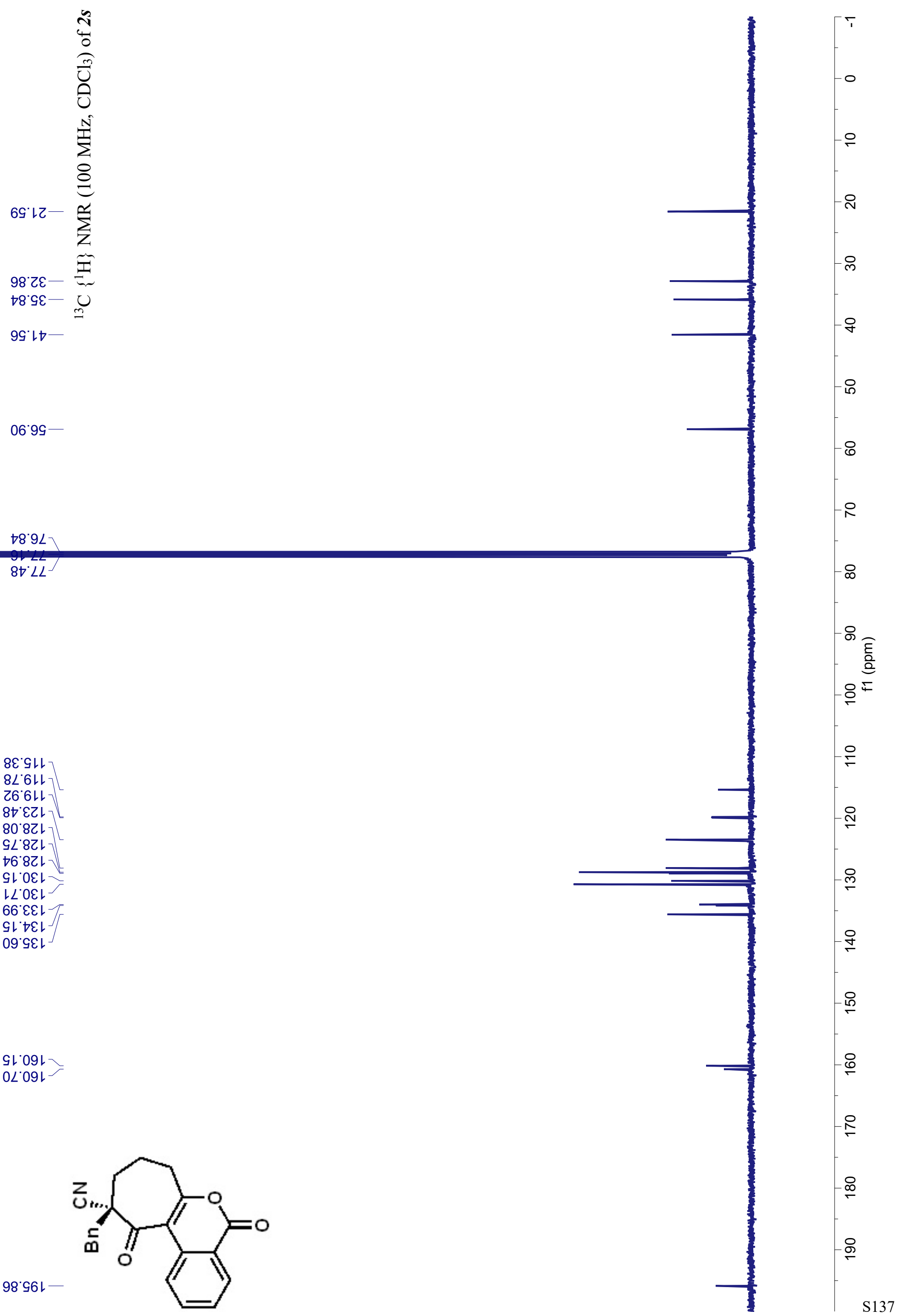


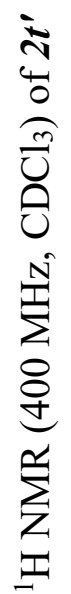

$\angle 8 L^{\circ} \varepsilon-$

乙6६ $\mathcal{E}^{\circ} \varepsilon-$

$\varepsilon+9 \cdot 9-$

$09 Z^{\circ} L$

てレ゙

6Lt L

$\checkmark Z \nabla^{\circ} L$

GEt $L$

OSt $L$

$0 \angle D^{\circ} L$

$06 t^{\circ} L$

$299^{\circ} \mathrm{L}$

$999^{\circ} \mathrm{L}$

$\varepsilon 89^{\circ} L$

$109^{\circ} \mathrm{L}$

$709^{\circ} \mathrm{L}$

$\varepsilon \nabla L$

$9 \nabla L L$

$Z 9 L ' L$

$99 L ' L$

$\forall L L \cdot L$

$18 L ' L$

$\checkmark 8 L^{\circ} L$

$90 \varepsilon^{\circ} 8$

$9 Z \varepsilon^{\circ} 8$

ติ 궁
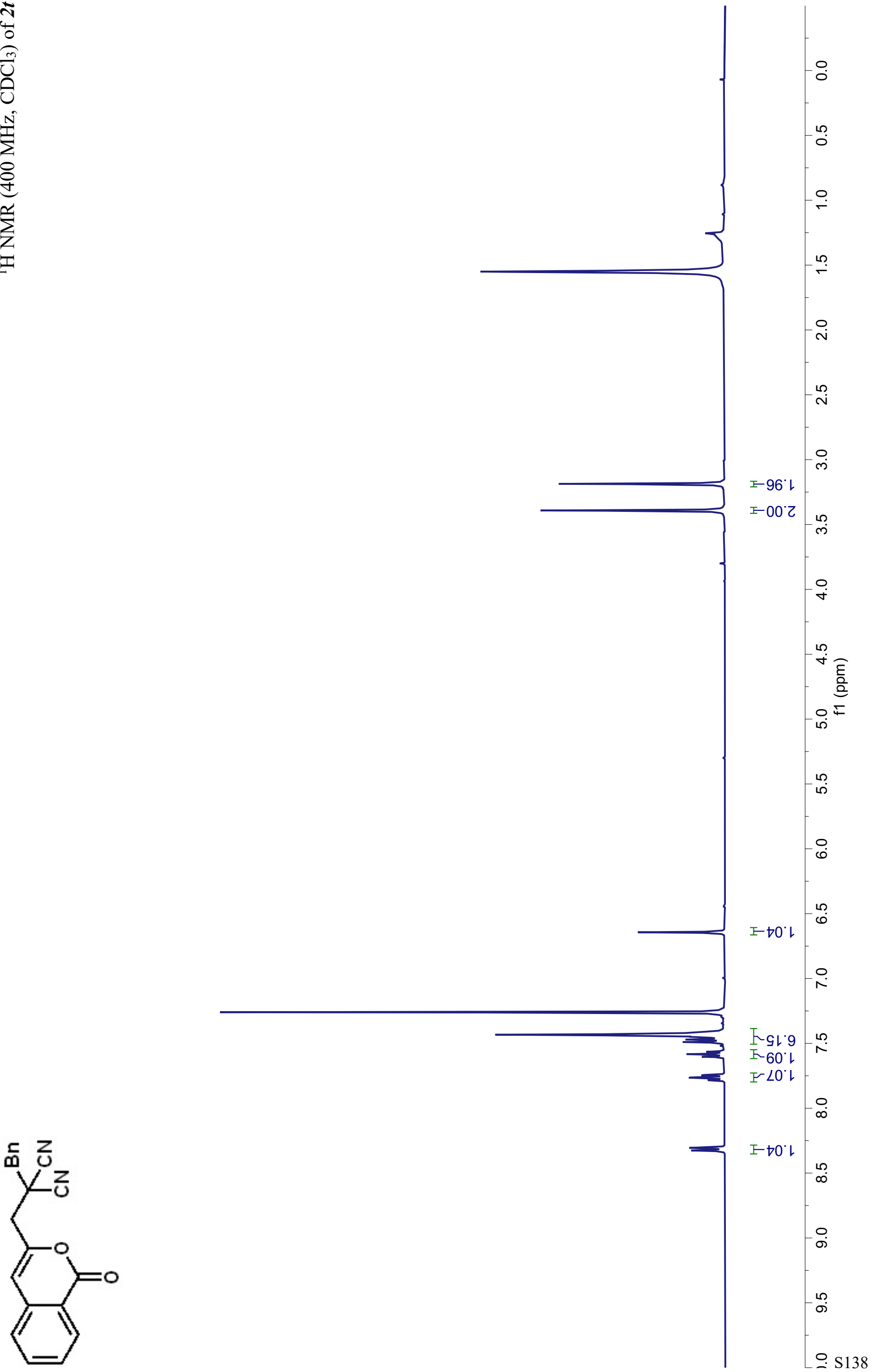


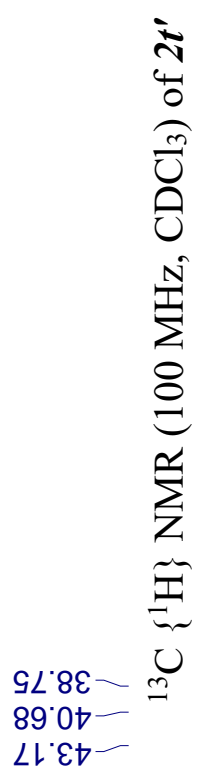

$78^{\circ} 9 L$

$8 \nabla^{\circ} L L$

89.80 -

งย๋レレ-

LOLZL

$\angle Z 9 Z \mathrm{~L}$

GZ6ZL

0ะ.6乙L

IS'6ZL

Z0००८

GG'0हL

LG'LEL

$\angle \mathcal{E} G \varepsilon L$

$60.9 \varepsilon L$

698カレー

Lナ๋เ9レ-
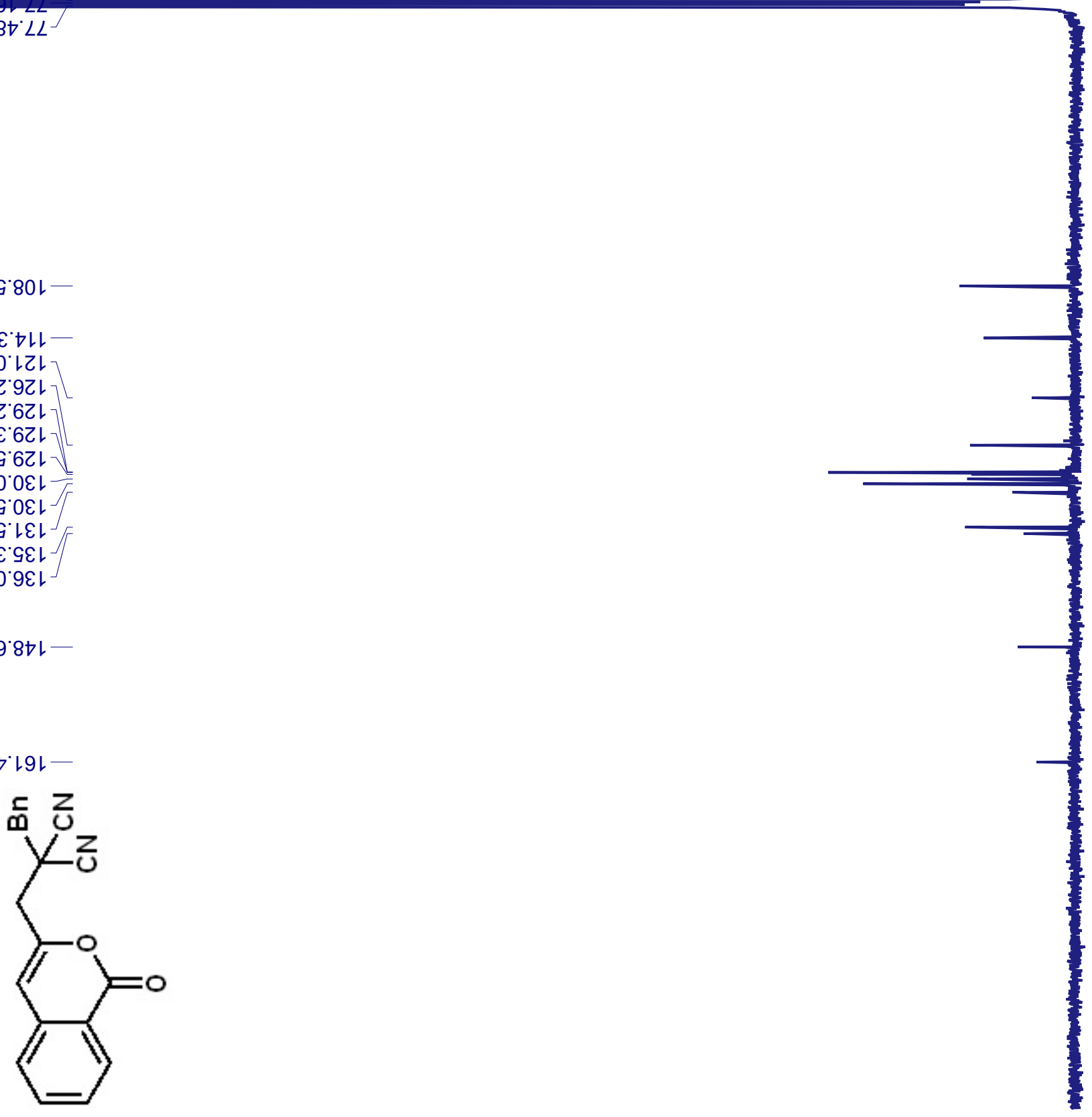


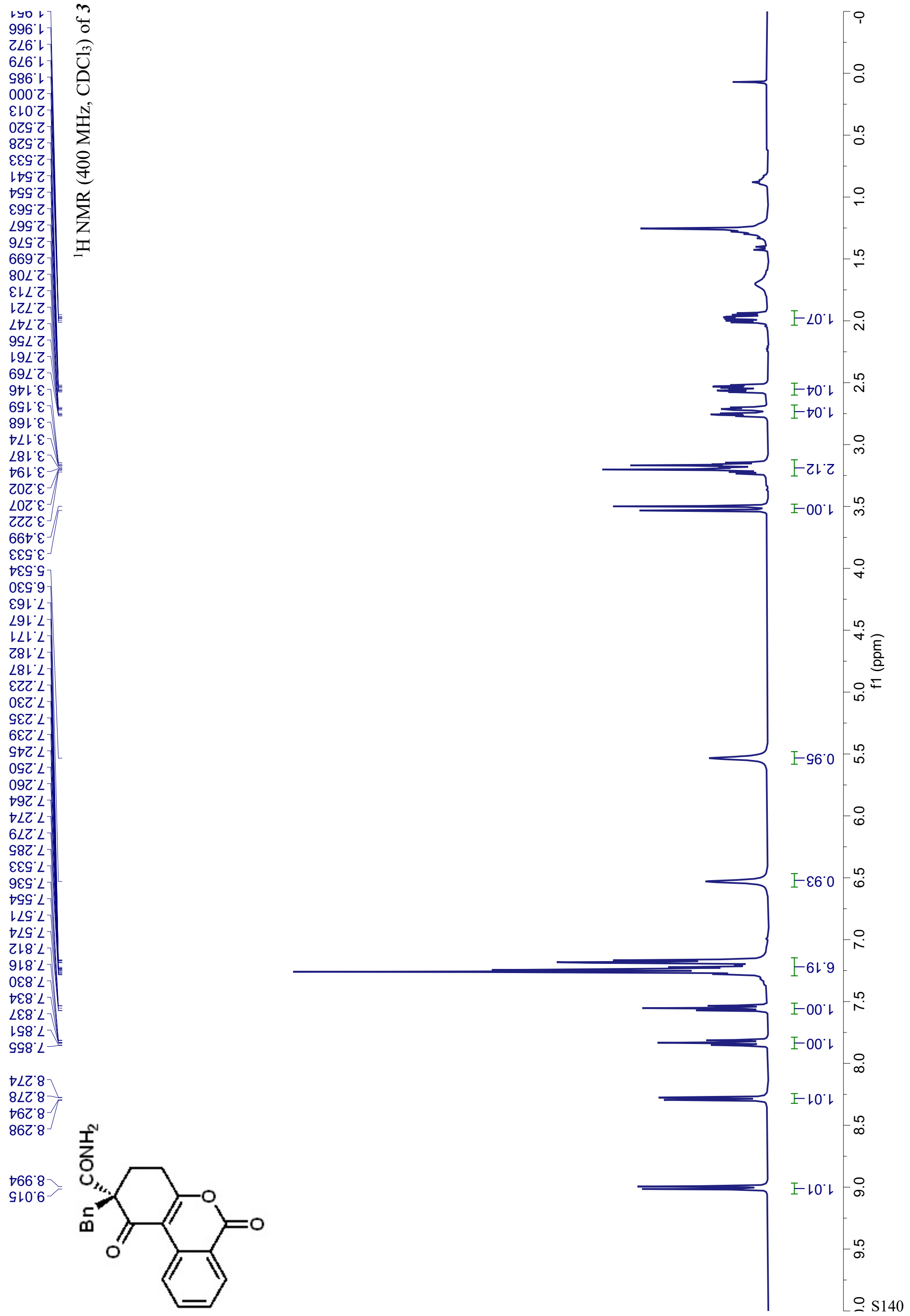




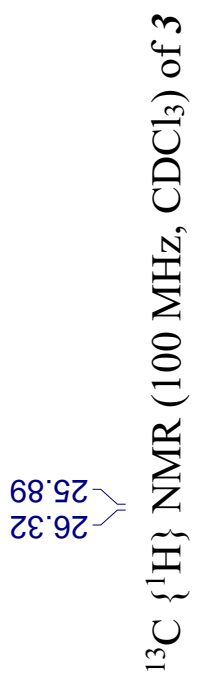

OS'Et-

$98.89-$

78.92

$9 L^{\circ} L L$

乙9・ルレ-

90.0ZL -

Z6.9ZL

$0 \nabla^{\circ} \angle Z L$

$\angle G^{\circ} 8 Z \mathrm{~L}$

08.82 L

†0 0 ㄴ

$6 \varepsilon^{\circ} 0 \varepsilon \mathrm{L}$

$\angle 8^{\circ} \varepsilon \varepsilon\llcorner$

L8 $9 E$ L

E0.09l-
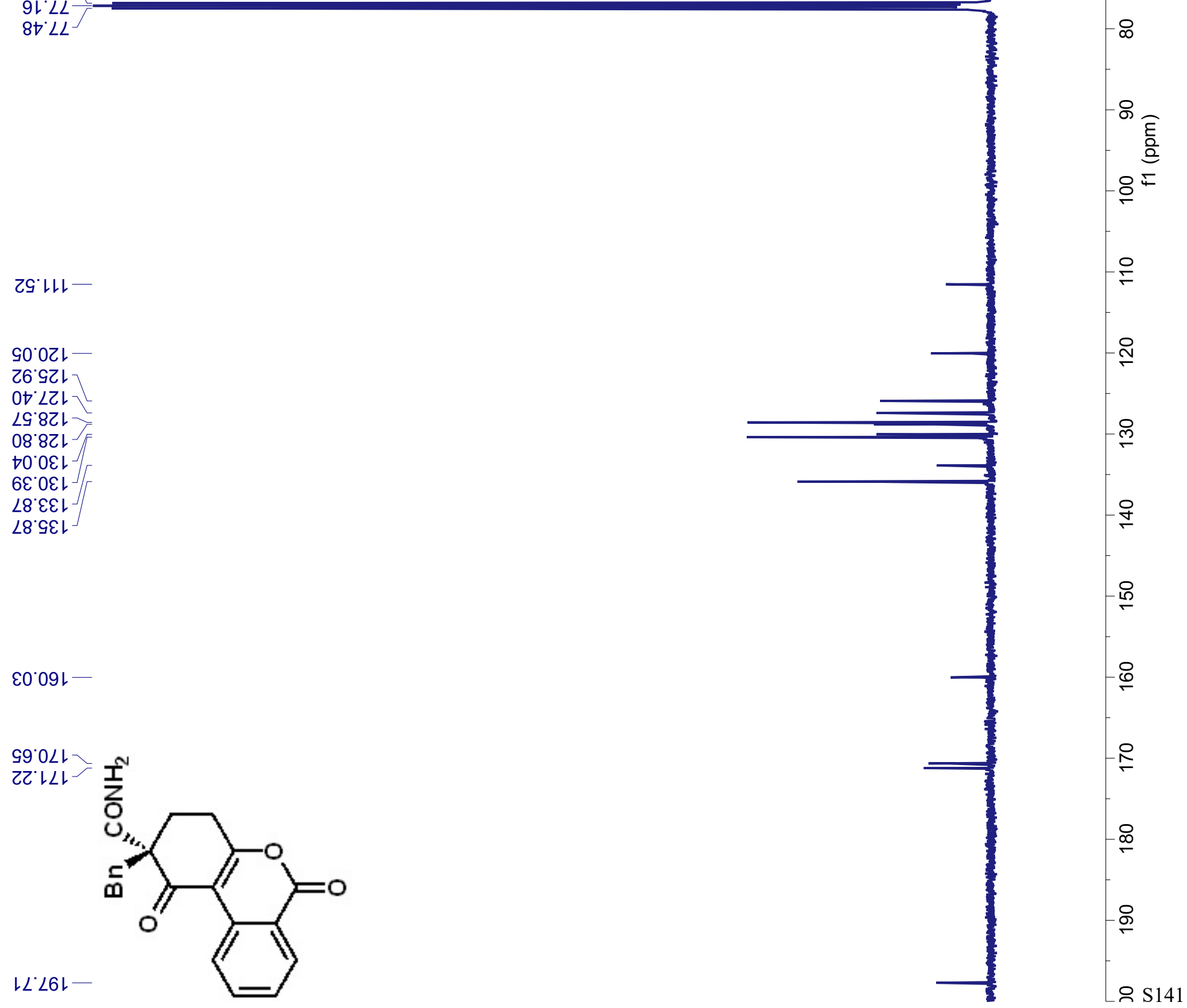


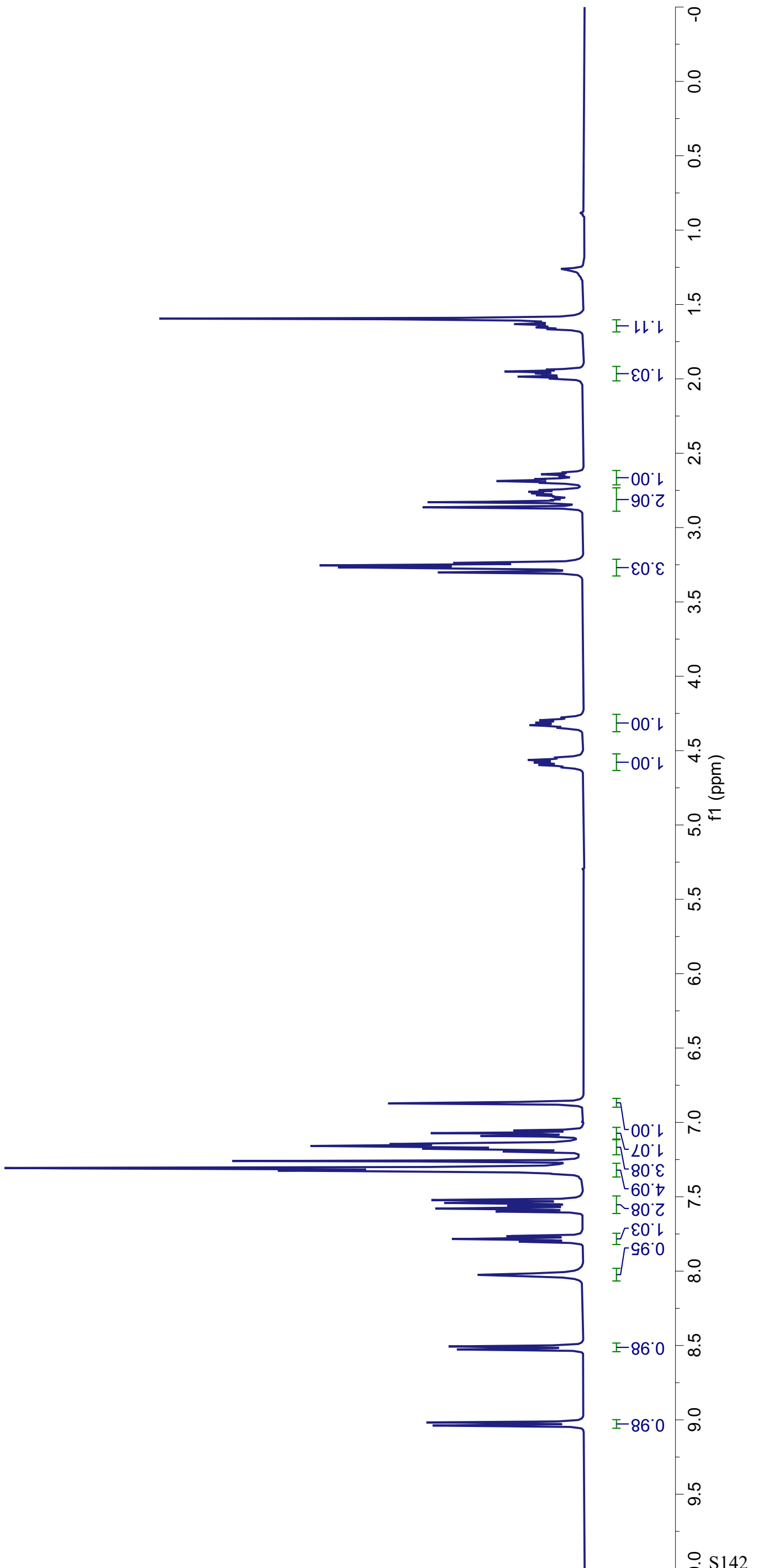




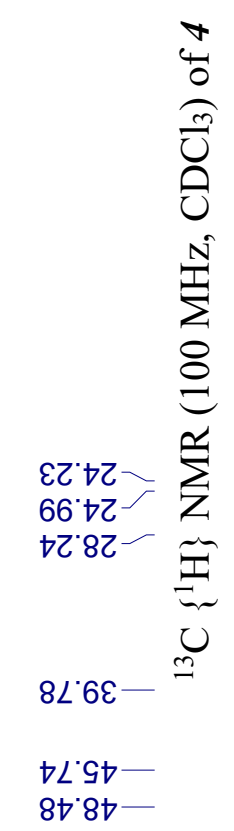

